NIST Special Publication 1900-603

\title{
NIST Transactive Energy Modeling and Simulation Challenge Phase II Final Report
}

David Holmberg, Martin Burns, Steven Bushby, Avi Gopstein Tom McDermott, Yingying Tang, Qiuhua Huang Annabelle Pratt, Mark Ruth, Fei Ding Yogesh Bichpuriya, Narayanan Rajagopal

Marija Ilic, Rupamathi Jaddivada

Himanshu Neema

This publication is available free of charge from:

https://doi.org/10.6028/NIST.SP.1900-603

\section{CYBER-PHYSICAL SYSTEMS}

National Institute of Standards and Technology

U.S. Department of Commerce 


\section{NIST Special Publication 1900-603}

\section{NIST Transactive Energy Modeling and Simulation Challenge Phase II Final Report}

David Holmberg, Martin Burns, Steven Bushby, Avi Gopstein National Institute of Standards and Technology, Gaithersburg, MD

Tom McDermott, Yingying Tang, Qiuhua Huang Pacific Northwest National Laboratory, Richland, WA Annabelle Pratt, Mark Ruth, Fei Ding National Renewable Energy Laboratory, Golden, CO Yogesh Bichpuriya, Narayanan Rajagopal Tata Consultancy Services, India

Marija Ilic, Rupamathi Jaddivada Massachusetts Institute of Technology, Boston, MA

Himanshu Neema Vanderbilt University, Nashville, TN

This publication is available free of charge from: https://doi.org/10.6028/NIST.SP.1900-603

May 2019

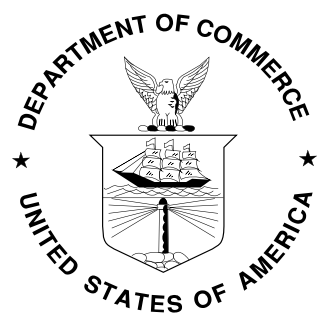

U.S. Department of Commerce Wilbur L. Ross, Jr., Secretary

National Institute of Standards and Technology Walter Copan, NIST Director and Undersecretary of Commerce for Standards and Technology 
Certain commercial entities, equipment, or materials may be identified in this document in order to describe an experimental procedure or concept adequately. Such identification is not intended to imply recommendation or endorsement by the National Institute of Standards and Technology, nor is it intended to imply that the entities, materials, or equipment are necessarily the best available for the purpose.

National Institute of Standards and Technology Special Publication 1900-603

Natl. Inst. Stand. Technol. Spec. Publ. 1900-603, 147 pages (May 2019)

CODEN: NSPUE2

This publication is available free of charge from:

https://doi.org/10.6028/NIST.SP.1900-603 


\begin{abstract}
The NIST Transactive Energy (TE) Modeling and Simulation Challenge for the Smart Grid (Challenge) spanned from 2015 to 2018. The TE Challenge was initiated to identify simulation tools and expertise that might be developed or combined in co-simulation platforms to enable the evaluation of transactive energy approaches. Phase I of the Challenge spanned 2015 to 2016, with team efforts that improved understanding of TE concepts, identified relevant simulation tools and co-simulation platforms, and inspired the development of a TE co-simulation abstract component model that paved the way for Phase II. The Phase II effort spanned Spring 2017 through Spring 2018, where the teams collaboratively developed a specific TE problem scenario, a common grid topology, and common reporting metrics to enable direct comparison of results from simulation of each team's TE approach for the defined scenario.
\end{abstract}

This report presents an overview of the TE Challenge, the TE abstract component model, and the common scenario. It also compiles the individual Challenge participants' research reports from Phase II. The common scenario involves a weather event impacting a distribution grid with very high penetration of photovoltaics, leading to voltage regulation challenges that are to be mitigated by TE methods. Four teams worked with this common scenario and different TE models to incentivize distributed resource response to voltage deviations, performing these simulations on different simulation platforms. A fifth team focused on a co-simulation platform that can be used for online TE simulations with existing co-simulation components.

The TE Challenge Phase II has advanced co-simulation modeling tools and platforms for TE system performance analysis, developed a referenceable TE scenario that can support ongoing comparative simulations, and demonstrated various TE approaches for managing voltage on a distribution grid with high penetration of photovoltaics.

\title{
Key words
}

co-simulation platform; modeling and simulation; smart grid; transactive energy; voltage regulation 


\section{Full Report Table of Contents}

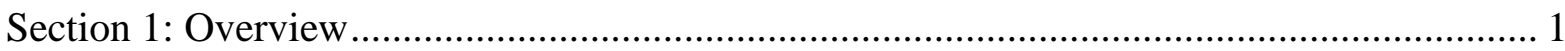

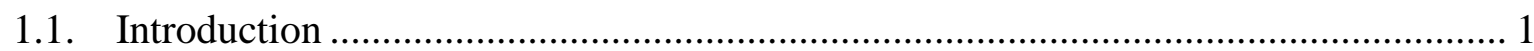

1.2. Transactive Energy and the NIST TE Challenge Phase I ....................................... 2

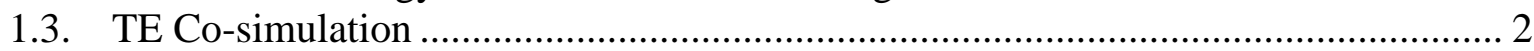

1.4. Common Challenge Scenario …………………………………………………….... 5

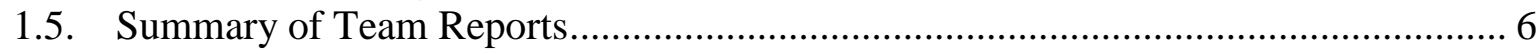

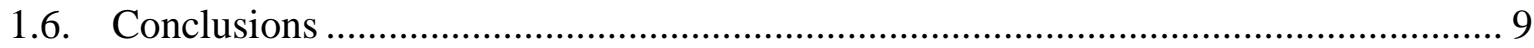

1.7. Overview References ........................................................................................... 11

Section 2 PNNL Team Report: Transactive Energy System Simulation for DER Integration

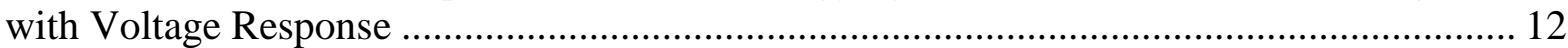

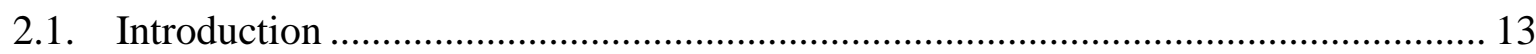

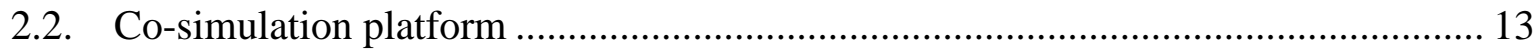

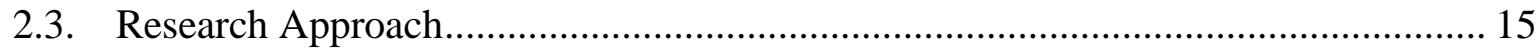

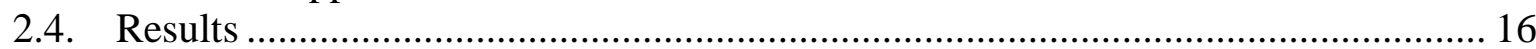

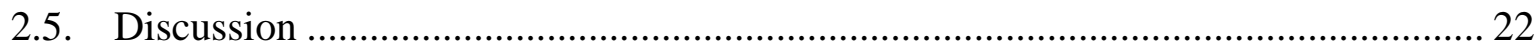

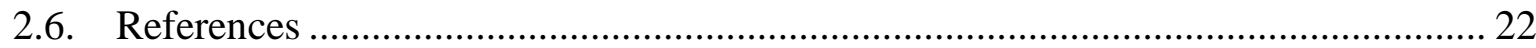

Section 3 NREL Team Report: Network-Aware Transactive Energy Control Simulations .. 23

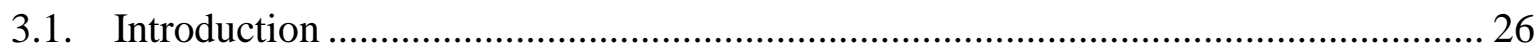

3.2. Co-Simulation Platform: Design and Interfaces Between Tools ............................... 27

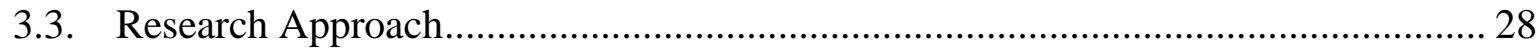

3.4. Transactive Energy Approach ............................................................................ 29

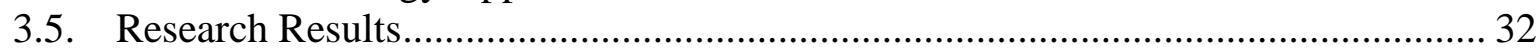

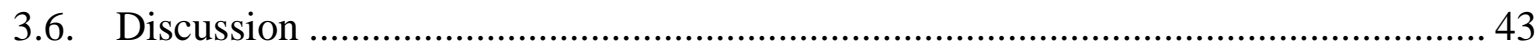

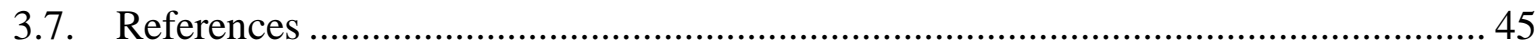

Section 4 TCS Team Report: Simulation of Dynamic Microgrid Based Operations in TE

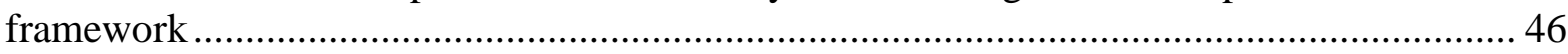

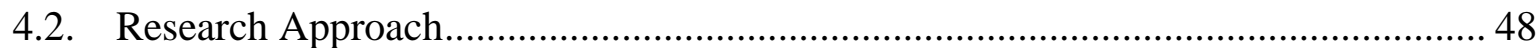

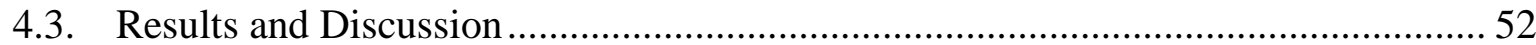

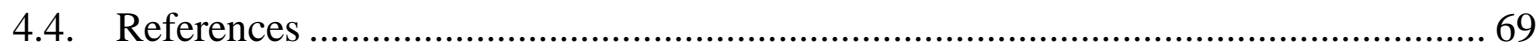

Section 5 MIT Team Report: Enabling operationally feasible prosumer-centric TEM ......... 70

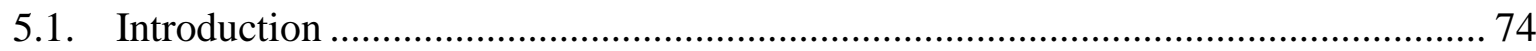

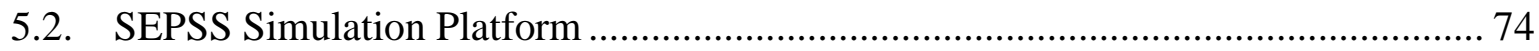

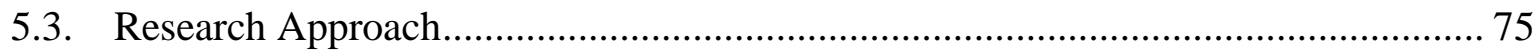

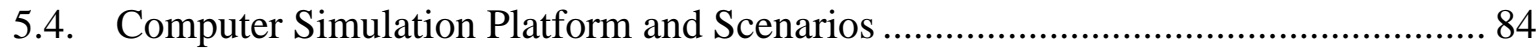

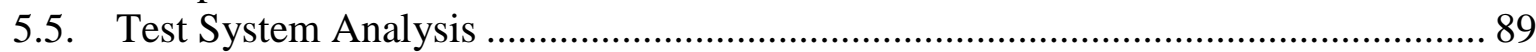

5.6. Future Utility Role in Transactive Energy Markets ................................................. 107

5.7. Preliminary Conclusions: Performance-based Grid Regulation based on TEM Peak

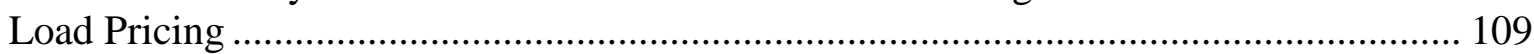

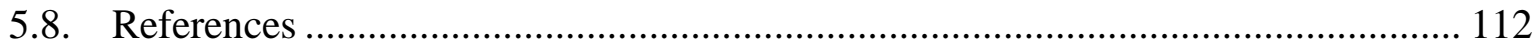

Section 6 Vanderbilt Team Report: Cyber-Physical Systems Wind Tunnel for Transactive

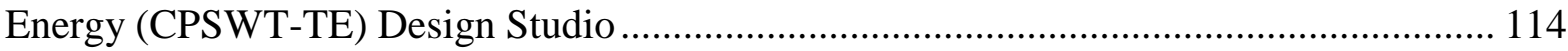

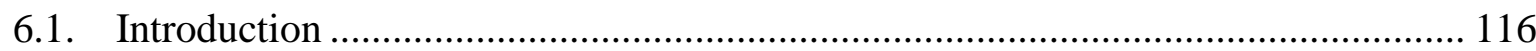

6.2. CPSWT-TE Design Studio Research Approach ................................................... 118

6.3. CPSWT-TE Design Studio Core Architectural Components................................... 120

6.4. CPSWT-TE Design Studio Experiment Development Workflow ............................. 123

6.5. GridLAB-D Modeling and Simulation Design Studio ............................................. 126

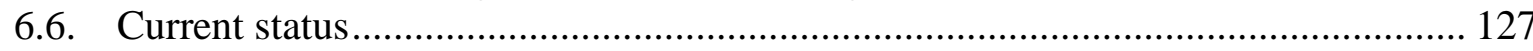




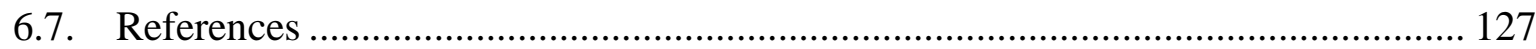

Appendix A TE Demonstration Projects in North America .............................................. 129

Appendix B TE Challenge Scenario Technical Specification ............................................ 133

\section{List of Figures}

Figure PNNL-1 Architecture of the co-simulation platform for NIST TE Challenge II ......... 13

Figure PNNL-2 Transactive market topology ................................................................. 14

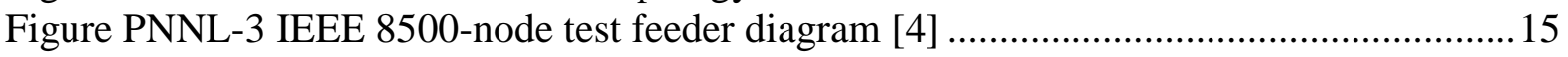

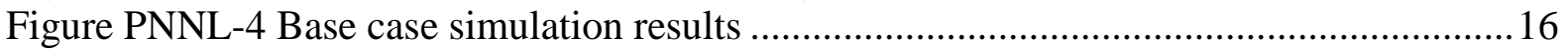

Figure PNNL-5 Bid and response of transactive ramp controller ...................................... 17

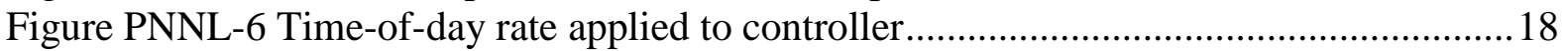

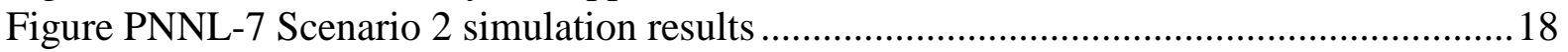

Figure PNNL-8 Voltage control at secondary side........................................................... 19

Figure PNNL-9 Relation between voltage change and power injection from the PV ............. 19

Figure PNNL-10 Scenario 3 simulation results ....................................................................20

Figure PNNL-11 Applied Volt-VAR (left) and Volt-Watt (right) functions; these are the defaults for category B ("high penetration") in IEEE 1547-2018.....................................20

Figure PNNL-12 Scenario 4 Volt-VAR mode simulation results ......................................21

Figure PNNL-13 Scenario 4 Volt-Watt mode simulation results .......................................21

Figure NREL-1. Diagram of the IESM including a co-simulation coordinator, simulated distribution feeder, simulated homes with home energy management systems, and markets. 27 Figure NREL-2 Mapping of IESM components to the abstract component model developed

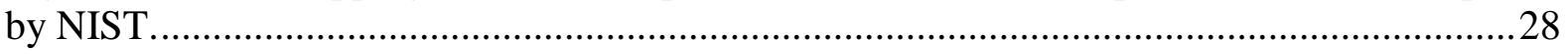

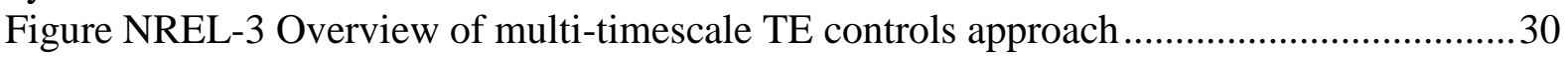

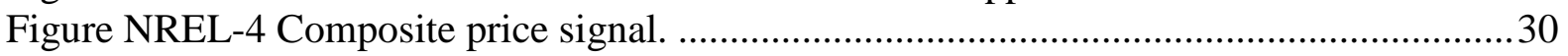

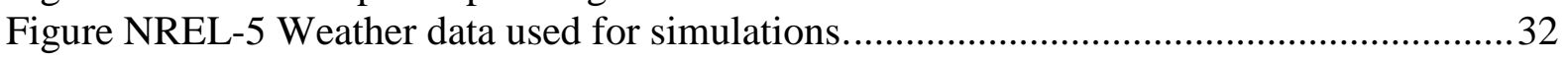

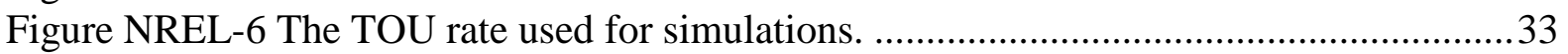

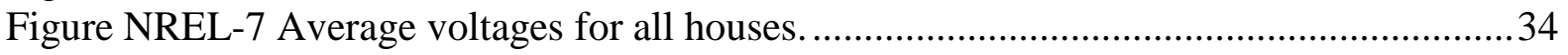

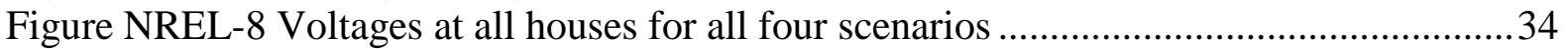

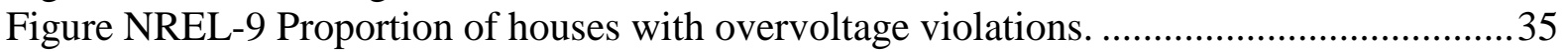

Figure NREL-10 Proportion of houses with undervoltage violations. ...................................36

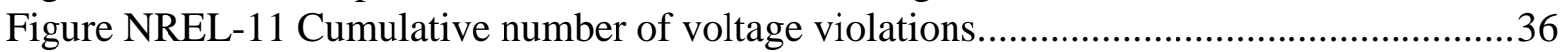

Figure NREL-12 Incentive prices for active power generated by the network-level control...37

Figure NREL-13 Incentive prices for reactive power generated by the network-level control.

Figure NREL-14 Average active (left) and reactive (right) net power consumed by houses... 38 Figure NREL-15 Average active (left) and reactive (right) power output of PV inverters.....38

Figure NREL-16 Average house air temperatures and average desired setpoint. ...................39

Figure NREL-17 Average power consumed by air conditioners.......................................39

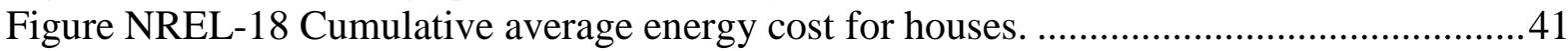

Figure NREL-19 Cumulative average energy cost components for the TEC scenario...........41 Figure NREL-20 Averages and ranges of energy costs for houses. The TEC scenario has four additional outliers that are not shown at: \$20, \$12.76, \$-9.56, and \$-27.22 .......................... 42 Figure NREL-21 Number of capacitor operations (left) and number of voltage regulator tap

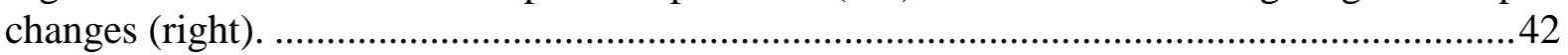

Figure NREL-22 Substation real (left) and reactive (right) power...................................... 43 
Figure TCS-1 Modified network of 8500 feeder system, truncated to three-phase nodes only

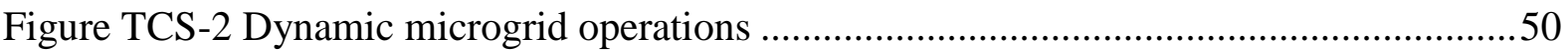

Figure TCS-3 Transactive energy market model ................................................................ 51

Figure TCS-4 Real power (Substation, House load and HVAC load) on a sunny day ...........52

Figure TCS-5 Solar PV power on a sunny day ................................................................53

Figure TCS-6 Max, Min and Average temperature of houses on a sunny day......................53

Figure TCS-7 Capacitor switching for base case on a sunny day......................................53

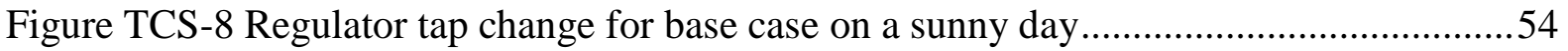

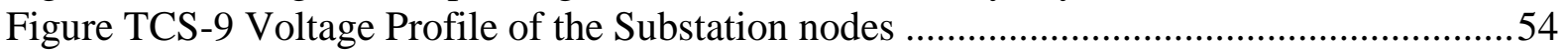

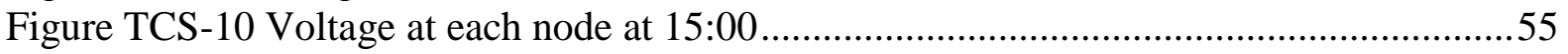

Figure TCS-11 Line Power Loss in 8500 Network ..............................................................55

Figure TCS-12 Real power (Substation, House load and HVAC) on a cloudy day ................56

Figure TCS-13 Solar PV power on a cloudy day ............................................................56

Figure TCS-14 Max, Min and Average house temperatures on a cloudy day ........................57

Figure TCS-15 Capacitor switching for base case on a cloudy day ......................................57

Figure TCS-16 Regulator tap change for base case on a cloudy day ...................................57

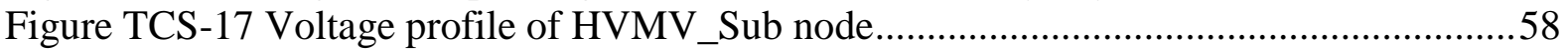

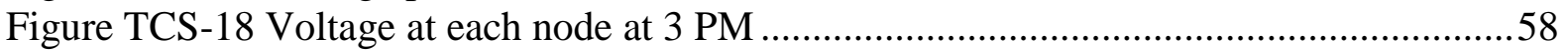

Figure TCS-19 Increase in Power drawn from grid during cloudy weather scenario .............59

Figure TCS-20 Substation real power of aggregated 8500 network..................................59

Figure TCS-21 Dynamic microgrid cluster formation in sunny scenario...............................60

Figure TCS-22 Dynamic microgrid cluster formation in cloudy scenario ............................61

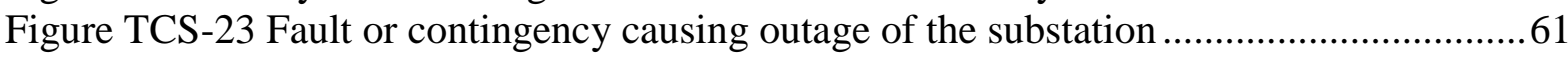

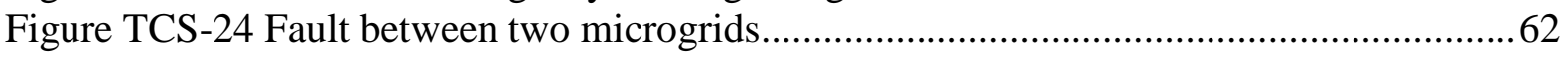

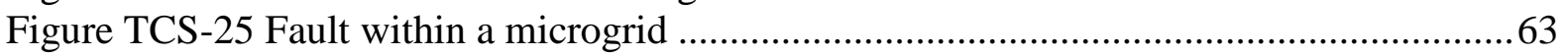

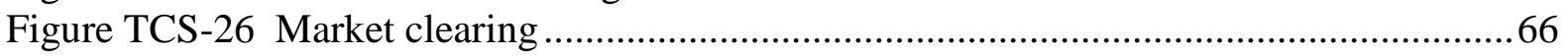

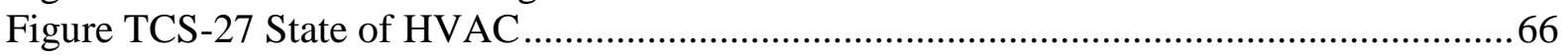

Figure TCS-28 Real power (Substation, House load and HVAC) on a cloudy day ................67

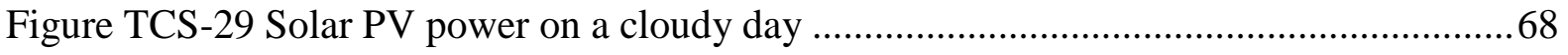

Figure TCS-30 Max, Min and Average temperature of houses on a cloudy day ....................68

Figure TCS-31 Real power (substation, house load and HVAC) on a sunny day..................68

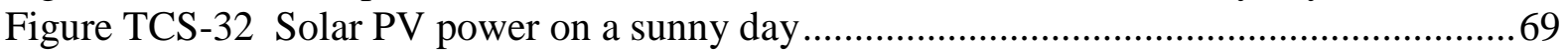

Figure TCS-33 Max, Min and Average temperatures of houses on a cloudy day ..................69

Figure MIT-1 Interaction of software modules within SEPSS computer platform [13] .........75

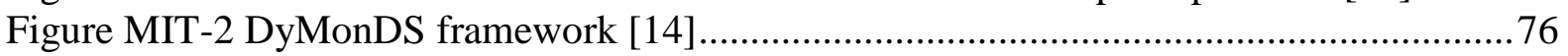

Figure MIT-3(a) Coupled droop of a water heater depicting the three-way relation ..............77

Figure MIT-3(b) Coupled droop of an inverter depicting the three-way relation ...................78

Figure MIT-4 Computer architecture for simulating the interactions needed for enabling TEM

.86

Figure MIT-5 Time series of the DER consumption patterns (negative consumption for solar

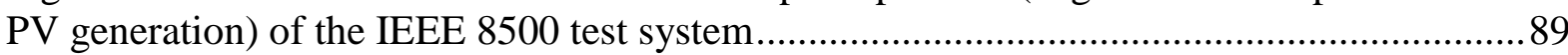

Figure MIT-6(a) Bid sensitivity of WHs to various relaxations.......................................... 92

Figure MIT-6(b) Bid sensitivity of HVACs to various relaxations ...................................... 92

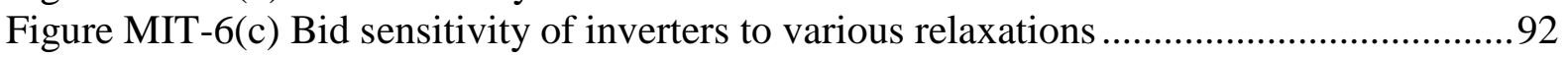

Figure MIT-7 Variable component of the tariff in tariff-driven pricing ..............................93

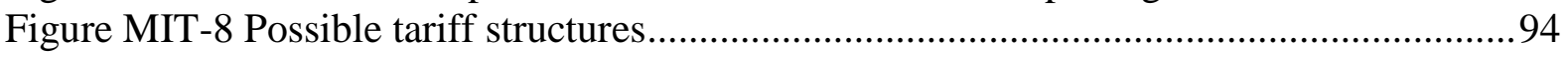

Figure MIT-9 Utility bus voltage magnitude .............................................................. 101

Figure MIT-10 Minimum and maximum voltages seen in the grid.................................... 101 


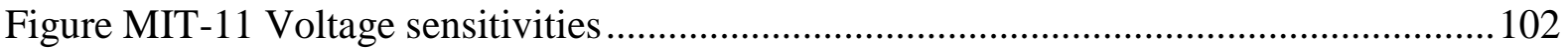

Figure MIT-12 Shunt capacitor reactive power injections at node 'R18242' ....................... 103

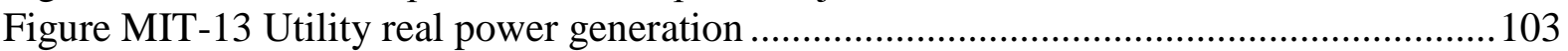

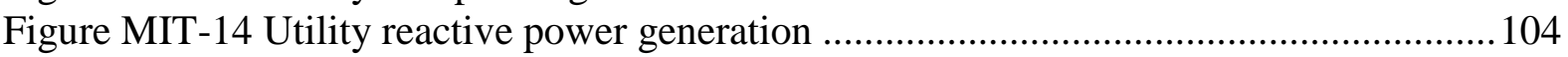

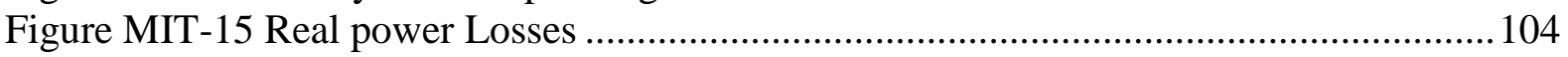

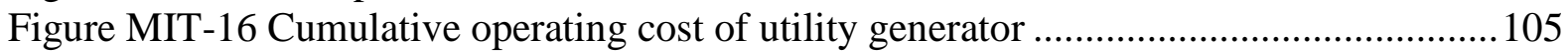

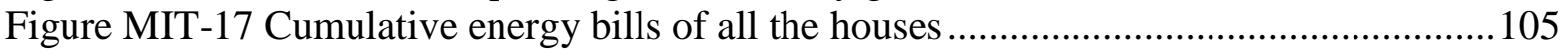

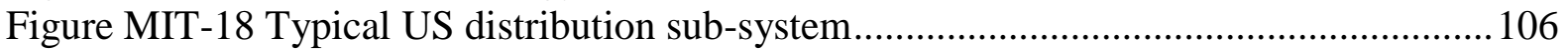

Figure MIT-19 Loading of children LV nodes coordinated by a single MV node................. 106

Figure MIT-20 Voltage drop across the service lines and transformers of the secondary

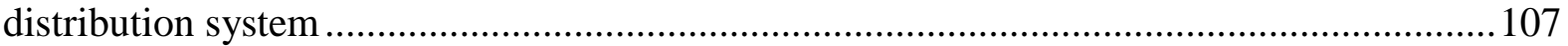

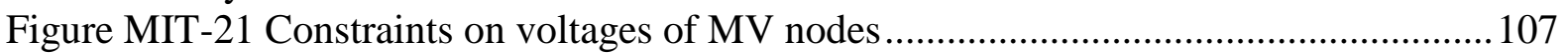

Figure Vanderbilt-1 Transactive Energy Concerns Targeted in CPSWT-TE ......................116

Figure Vanderbilt-2 CPSWT-TE Platform Architecture ................................................ 119

Figure Vanderbilt-3 CPSWT-TE Design Studio Architectural Components (Source: [14]) 120

Figure Vanderbilt-4 Experiment Specification Example .................................................. 122

Figure Vanderbilt-5 CPSWT-TE Design Studio Experiment Development Workflow (Source

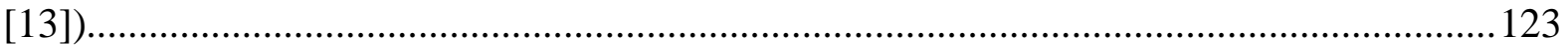

Figure Vanderbilt-6 CPS-VO Experimentation Interface for the CPSWT-TE Design Studio

Figure Apdx B-1 IEEE 8500 grid schematic.

134

\section{Table of Tables}

Table PPNL-1 Customized IEEE 8500-node feeder properties ......................................... 15

Table PNNL-2 Parameters in controller bid and response curve ...................................... 17

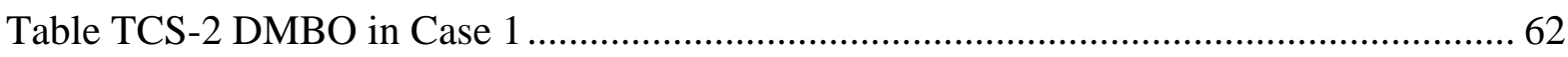

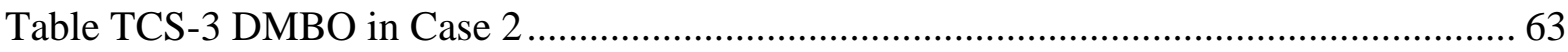

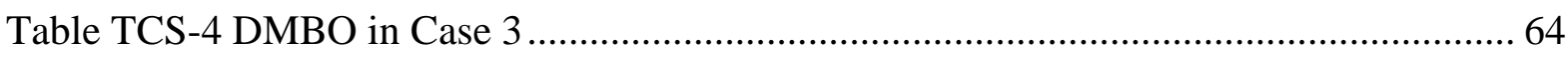

Table TCS-5 Comparison of Load Interruptions without and with DMC .............................. 64

Table MIT-1 Roles and Performance metrics in TEC cases................................................. 84

Table MIT-2 Power generation/ Consumption metrics ........................................................ 96

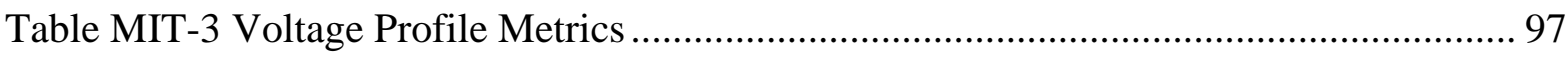

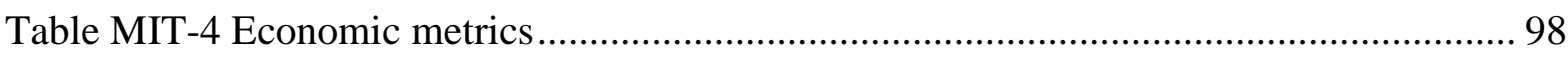

Table Apdx B-1 Tucson Electric Residential Basic Service Tariff .................................... 135

Table Apdx B-2 Tucson Electric Residential Service Time-of-Use Tariff ......................... 135 


\section{Acronyms and Abbreviations}

3Rs

$\mathrm{AC}$

rules, rights and responsibilities

$\mathrm{A} / \mathrm{C}$

alternating current

$\mathrm{ACM}$

air conditioner

ACOPF

ANSI

BESS

BPS

CPS

Abstract Component Model

alternating current optimal power flow

CPSWT-TE Cyber-Physical Systems Wind Tunnel for Transactive Energy

CPS-VO CPS Virtual Organization

DER American National Standards Institute

battery energy storage system

bulk power system

cyber-physical systems

DERMS distributed energy resource management system

DLMP distributed locational marginal price

DM dynamic microgrids

DMC Dynamic Microgrid Configurator

DMBO dynamic microgrid based operations

DMS distribution management system

DOE U.S. Department of Energy

DPS distribution power systems

DSO distribution system operator

DTU Technical University of Denmark

DyMonDS Dynamic Monitoring and Decision Systems

ESIF Energy Systems Integration Facility

EV

FLOPS floating point operations per second

FNCS

HEMS

HLA Framework for Network Co-Simulation

HPC home energy management system

HVAC

High Level Architecture

IEEE

high-performance computing

IESM

ISO

LMP

LV

MCP

MCQ

MIT

MPC

MV heating, ventilating and air conditioning

Institute of Electrical and Electronics Engineers

medium voltage

NIST National Institute of Standards and Technology

NREL National Renewable Energy Laboratory

NW

OPF

NETSSWorks

PNNL Pacific Northwest National Laboratory

PoC point of connection

PLP peak-load pricing

PRD price-responsive demand 


$\begin{array}{ll}\text { p.u. } & \text { per unit } \\ \text { PSEC } & \text { Power Systems Engineering Center } \\ \text { PV } & \text { photovoltaic } \\ \text { QoS } & \text { quality of service } \\ \text { SCADA } & \text { supervisory control and data acquisition } \\ \text { SEAC } & \text { Strategic Energy Analysis Center } \\ \text { SEPSS } & \text { Scalable Electric Power System Simulator } \\ \text { SGRS } & \text { Smart Grid in a Room Simulator } \\ \text { TCS } & \text { Tata Consultancy Services } \\ \text { TE } & \text { transactive energy } \\ \text { TE Model } & \text { NIST TE Co-simulation Abstract Component Model } \\ \text { TEC } & \text { (NREL) transactive energy control } \\ & \text { (MIT) TE Challenge } \\ \text { TEM } & \text { Transactive Energy Management } \\ \text { TESP } & \text { Transactive Energy Simulation Platform } \\ \text { TMY } & \text { typical meteorological year } \\ \text { TOU } & \text { time of use } \\ \text { VAR } & \text { volt amp reactive } \\ \text { WH } & \text { water heater } \\ \text { ZIP } & \text { constant impedance (Z), constant current (I), constant power (P) load } \\ \text { ZMQ } & \text { ZeroMQ }\end{array}$




\section{Section 1: Overview}

\subsection{Introduction}

The evolving smart grid, with increased use of renewable energy generation and distributed energy management technologies, is changing the way electricity is managed and the relationship between the grid and energy consumers. As consumers gain the ability to produce electricity, market-based transactive exchanges between energy producers and energy consumers offer the potential for significant grid efficiency and reliability improvements.

A number of transactive energy (TE) demonstrations have been carried out, as reviewed in Appendix A, to test the effectiveness of different transactive market configurations and their ability to address grid problems and provide customer benefits. Demonstrations are expensive, time consuming, and the results are difficult to compare.

To understand the potential of TE and to support technology developers and policy makers, the smart grid community requires simulation tools and co-simulation platforms that can be used to explore the benefits and impacts of alternative ways to create and operate TE systems. Compared to real-world demonstrations, simulation experiments enable the evaluation of multiple TE approaches with different grid conditions, potentially on the same grid, over a wider range of input variables. The TE Challenge was conceived with the goal of advancing simulation tools and platforms for TE system evaluations.

Simulation of a transactive system for a specific grid and market architecture, with various actors producing and consuming energy and other grid services, and for a given weather and event scenario, is a complex co-simulation challenge. Information must be exchanged between simulation components as an experiment progresses to enable successful evaluation of TE system economic and technical metrics. The TE Challenge Phase II was designed as a co-simulation exercise that limited complexity while providing common elements to enable comparison of different co-simulation tools evaluating different TE approaches. This was made possible by the collaborative development and agreement among teams on a common scenario with a common grid, and common metrics for evaluating results.

Five teams participated in the 2017-2018 TE Challenge Phase II. This report collects the work of these teams. The products of the Challenge will help industry better understand the capability, repeatability, and utility of simulation platforms that can be used to study the impact of different TE approaches. The knowledge gained can provide input to guide next steps for real-world implementations.

The goals for Phase II of the TE Challenge were the following.

1. Perform TE simulations using a collaboratively developed TE scenario that serves as a baseline for comparisons of simulation results.

2. Develop simulation-platform-agnostic common understandings and interoperable TE modeling approaches that will allow the broad community to incorporate transactive elements into their own analyses.

3. Build up the TE community and promote collaborations that can support efforts to advance TE implementations.

4. Provide visibility for different co-simulation platforms and understanding of strengths for each.

This report reviews the common scenario used by the Phase II teams and collects the individual team reports. 


\subsection{Transactive Energy and the NIST TE Challenge Phase I}

Transactive Energy has been defined [1] as "a system of economic and control mechanisms that allows the dynamic balance of supply and demand across the entire electrical infrastructure using value as a key operational parameter." The NIST TE Challenge has brought a community of researchers and practitioners together to demonstrate the application of TE approaches to real grid problems using diverse modeling and simulation platforms.

NIST has worked in coordination with the U.S. Department of Energy to explore the potential of TE to improve the safety, efficiency, reliability, resiliency, and adaptability of the grid. The NIST TE Challenge was designed to facilitate the application of common TE principles that can be explored with integrity across diverse modeling simulation toolsets, thereby allowing the broad community of electric grid and systems modelers to incorporate transactive elements into their own analyses. To accomplish this, NIST engaged organizations to develop simulation-platform-agnostic common understandings and interoperable approaches to TE modeling.

Seven teams participated in the TE Challenge Phase I (2015-2016) with outputs including: definition of TE scenarios, development of a TE common abstract component model for TE co-simulation [2], analysis of the TE regulatory environment, and analysis of common transactive services. Information about the Phase I teams and the work products produced by these teams are available in the Phase I final report [3].

Development of a TE Co-simulation Abstract Component Model [2] (TE Model) was foundational to the TE Challenge Phase II effort. The TE Model defines a set of component building blocks (energy grid, weather, loads, generators, controllers, and transactive agents) that can be combined and duplicated to describe a TE scenario. The goal of the TE Model is to enable understanding, discussion, evaluation and validation of any TE approach. The detailed TE Model specification was designed to allow implementation on one or more simulation platforms. It serves as a reference model for TE co-simulation and it is expected that over time there will be data sets, behavior models, common starting tools, message sets, communications modeling tools and other supporting tools developed on the TE Model so that any stakeholder has a starting toolbox from which to tinker.

\subsection{TE Co-simulation}

Co-simulation is the process by which separate simulation programs are operated in concert to produce a combined result. It is common for any industry to have tools to simulate and evaluate various aspects of the domain of interest. It is often the case in the interconnected world of cyber-physical systems (CPS) and Internet of Things (IoT) that it becomes useful for such domain-specific tools to be joined together.

As part of the Phase I work, collaborative research was performed to abstract the components of any transactive approach, captured in the TE Model referenced above. By this means, specializations of the abstract components may be constructed that could be run on compatible platforms and thereby compared to other such specializations.

The TE Model specifies the key components of a TE co-simulation experiment, the interfaces between the components, and the information elements to be exposed and communicated. The TE Model is abstract. It does not constrain the implementation details for exchanging data between components and it permits the combining of components. The TE Model serves as a tool for establishing a common understanding of the basic building blocks and information exchange that enables developers to implement components that can interoperate with others in a co-simulation. Figure NIST-1 gives one example of representing the actors in a transactive energy market using the model building blocks. 

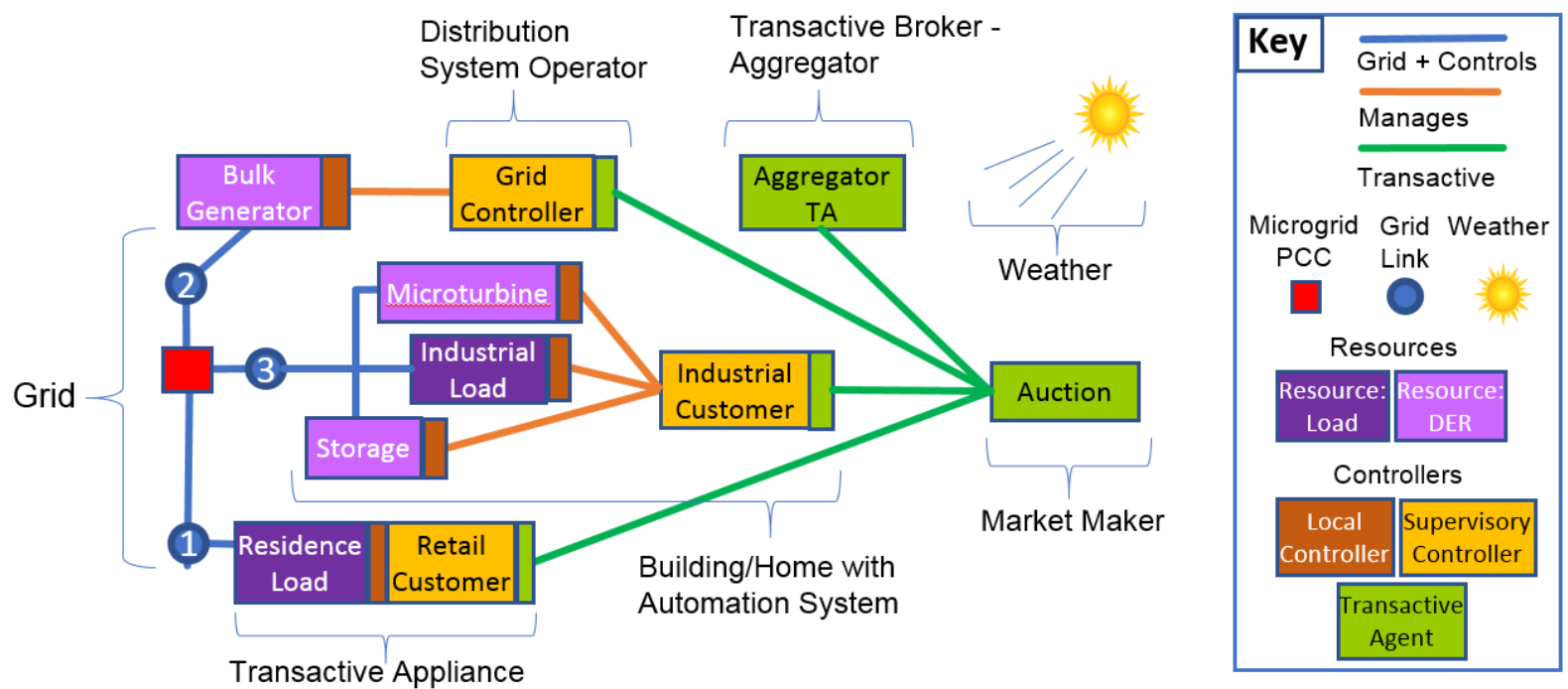

Figure NIST-1 Notional topology of the Abstract Component Model

At the heart of the model is the simulation Grid component which represents the electrical distribution system, for which there exists a great deal of modeling expertise. For this transactive energy abstraction, the grid represents the entirety of connected devices responsible for electricity delivery and operation. It only exposes the links and nodes to which transactive energy resources are attached. Load and DER Resource components consist of loads and generators (including storage) that sink or source energy.

There are two types of controllers. The Local Controller is a component that understands the nature of a resource. A thermostat is a good example of a local controller for an HVAC system load. The component model concentrates the physical nature of resources in the resource definition and the logical part of the component in the local controller. This allows the "physical part" to interact with the physics of the grid simulation while the Local Controller can interact with the Supervisory Controller, in a higher abstraction of supervisory control. The Supervisory Controller coordinates its attached resources for optimized grid interaction, has consumer inputs (setpoints and schedules) and aggregates DER forecasts up to a Transactive Agent.

The Transactive Agent is typically tightly coupled to the supervisory controllers and is responsible for market interactions. Finally, a Weather component is responsible for providing the changing environment that drives energy production and consumption.

This modular structure enables stakeholders to conduct experiments by building on the implementations of other researchers, substituting components as needed to enable exploration of a different research objective. For example, while dynamic load and economics may be the focus of some, transient behaviors, capital life cycles and degradations, or susceptibility to malicious manipulation may be the focus of others. With the existence of comparative platforms and example implementations of offered TE ideas, researchers can examine these ideas independently of their creators. The definition of a common abstract component model, therefore, will lead to platforms where the goals of comparative TE simulations can be realized.

Figure NIST-2 shows how these components can be combined in a "canonical experiment". With this concept, it is possible to perform simulations of any arbitrary set of versions of the abstract model components and apply a uniform set of metrics for comparison.

These modeling concepts guided the Phase II project teams and provided the basis for shared understanding of co-simulation models. 


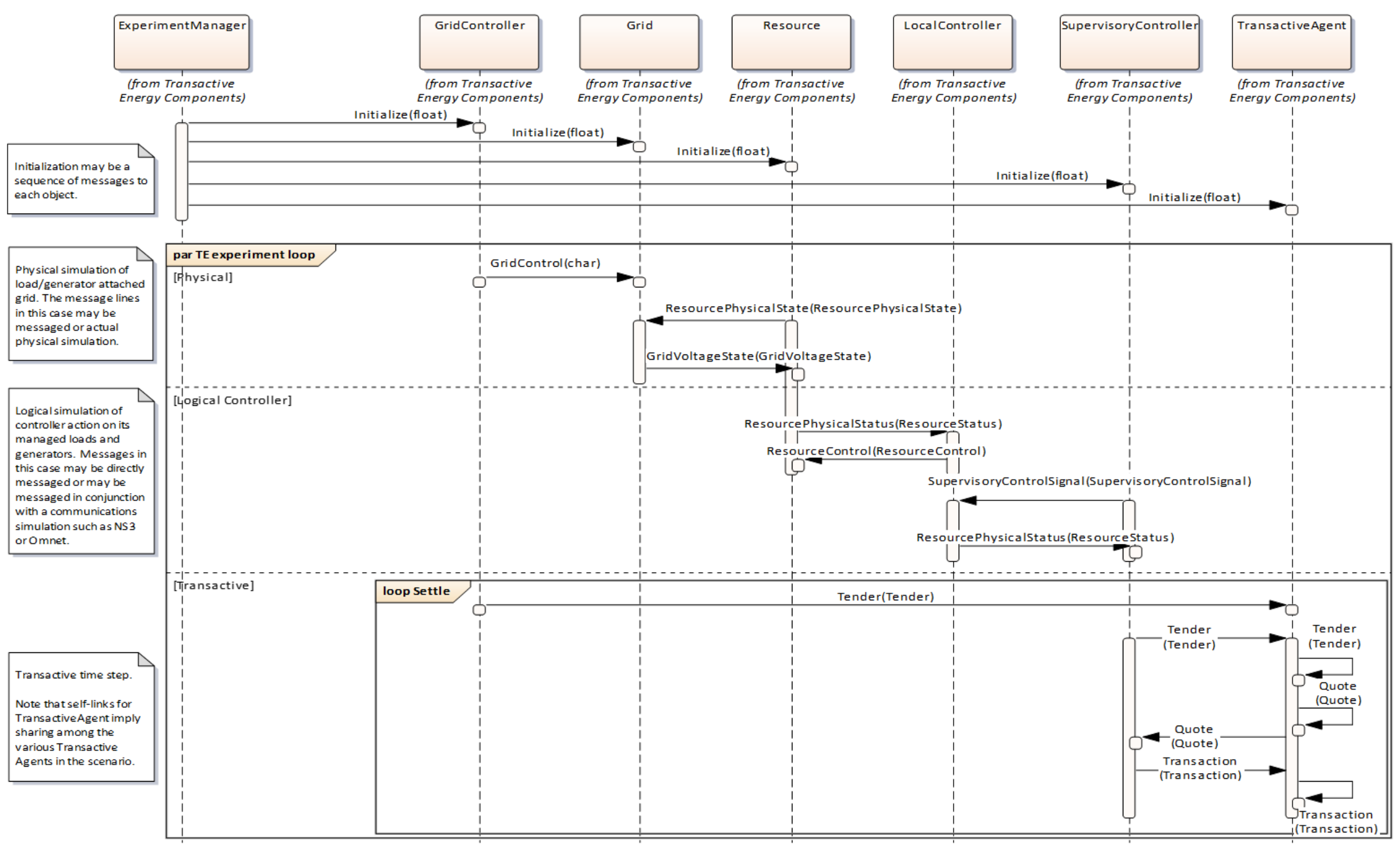

Figure NIST-2 Abstract Component Model standardized experiment 


\subsection{Common Challenge Scenario}

As documented in detail in the TE Challenge Phase II Scenario report [4], the collaborative process of developing the Challenge Scenario enabled a shared understanding among participants of each team's co-simulation platforms, simulation goals, and TE approaches. The resulting scenario components can serve as a reference resource for others to compare results from their own TE simulations with different TE approaches. The technical details of the Scenario are included in Appendix B of this report. An introduction is presented here.

The Challenge Scenario is a collection of components including: a specific electric feeder with specified loads and generators, an event sequence (weather for one day), a time-of-use price schedule for the baseline (non-TE) case, a series of simulations that move from non-TE baseline up to simulations with TE models chosen by each team, and an agreed upon set of common metrics for reporting results.

The use case scenario focuses on voltage imbalances due to high-penetration of photovoltaic (PV) systems. The teams chose to use a large feeder model based on the IEEE 8500 reference grid [5] shown in Figure NIST-3. This choice provides a diversity of loads and generation with potential for voltage variation across the feeder due to PV concentrations. To reduce complexity of the co-simulation exercise, teams agreed not to model the transmission grid. The grid model is populated with approximately 2,000 homes, each with controllable air conditioning systems and uncontrolled plug loads, while $90 \%$ have PV systems, and $50 \%$ have electric hot water heaters.

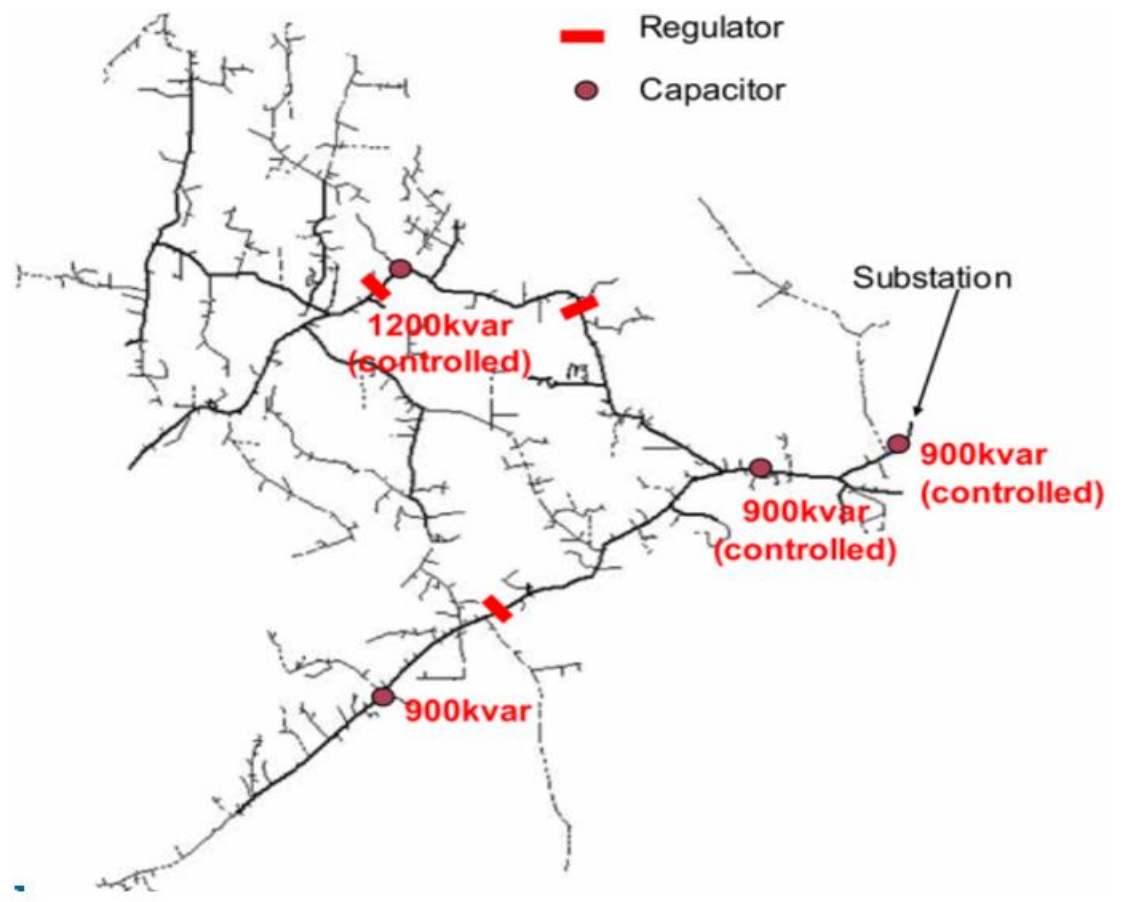

Figure NIST-3 IEEE 8500 reference grid [5] schematic.

The simulation day includes a passing storm front that results in solar irradiation being severely reduced in the 2:30-4:30 p.m. time window. Different teams used different approaches to manage the voltage swings, but teams generally followed the same experiment sequence: baseline sunny day with flat price; storm front added; time-of-use (TOU) price variation added, TE model introduced to manage device operation to reduce voltage fluctuations. 
Pricing and tariff data were agreed upon for the above experiments. Weather data (temperature, solar insolation, humidity, pressure and wind speed) were provided.

The final component of the common scenario was the set of metrics used for data reporting. These metrics include voltages (real and reactive) at grid nodes including phase-to-phase voltages, control actuations at voltage regulators and capacitor banks, voltage violations, prices for any transactions, cost and revenue at each house meter, power at each load, house and water heater temperatures, and local use of renewable power at the feeder level.

Additional details about metrics, weather, and grid configuration assumptions are included in Appendix B. An executive summary of Team experimental results is presented below. Complete team reports are presented in Sections 2 through 6.

\subsection{Summary of Team Reports}

Five teams participated in the TE Challenge Phase II, helping to develop the common scenario, then carrying out the co-simulation experiments. The teams were: Pacific Northwest National Laboratory (PNNL), National Renewable Energy Laboratory (NREL), Tata Consultancy Services (TCS), Massachusetts Institute of Technology (MIT), and Vanderbilt University. An executive summary of each team's work is presented here.

\subsubsection{Pacific Northwest National Laboratory}

PNNL developed detailed representations of the baseline IEEE 8500 grid including specific loads, generation, house models and device controllers. These were shared with the other teams who used these configurations in their own simulations. PNNL also provided the weather data and price scenarios used by the Challenge teams. Finally, PNNL provided baseline results used for comparison by each team. Baseline results were obtained for sunny and cloudy non-TE grid operations with flat price and TOU price inputs.

Using GridLAB-D and their TE Simulation Platform, which was under development during the TE Challenge period, PNNL examined the impacts of HVAC temperature setbacks and inverter Volt-VAR and Volt-Watt controls to manage distribution grid voltage violations. They chose to study this separately from a TE market. They made this choice based on the recognition that some voltage fluctuations happen faster than the response capability of a five minute market cycle.

Their results show a strong potential for load shifting and PV system real and reactive power adjustments to provide local voltage control on the distribution grid. Volt-VAR control, which initiates reactive power production (or consumption) as voltage deviates from normal before it reaches violation limits, was found to be most effective at helping to keep voltage peaks from exceeding violation limits. Volt-VAR control reduced voltage violation duration to $222 \mathrm{~h}$, as compared to the baseline $574 \mathrm{~h}$. Volt-Watt control initiates real power reduction at the PV inverter only after a violation limit is reached. Volt-Watt controls reduced voltage violation duration to $553 \mathrm{~h}$, with a reduction of PV system output of $0.75 \%$. HVAC set point adjustment was implemented by reducing set point temperature $4^{\circ} \mathrm{F}\left(2^{\circ} \mathrm{C}\right)$ when upper voltage violation limits were reached to help absorb over-generation from PV systems. Use of HVAC load shifting resulted in voltage violation duration of $464 \mathrm{~h}$.

\subsubsection{National Renewable Energy Laboratory}

NREL implemented a TE control approach to reduce the number of voltage violations, specifically overvoltages caused by generation from rooftop PV systems. They demonstrate how both smart PV inverters as distributed generation, and air conditioners with smart thermostats as flexible load, can be incentivized to participate in TE controls to improve voltage performance. 
The impact of changes in PV generation or air conditioner power consumption on the voltage depends on the location of the home that contains these devices within the power system network. Therefore, NREL implemented location-specific price signals to incentivize homes to adjust their power, i.e., each house receives a unique price signal. This requires the calculation of these price signals to incorporate the network topology, i.e., the controls need to be "network-aware." NREL implemented a network-aware TE control approach that enables the management of distribution feeder voltages based on real-time optimal power flow (OPF). A distribution network controller monitors voltages at each house, estimates future power usage and flexibility of homes, runs a power flow model, and uses this information to generate per-house incentive signals for both real and reactive power because both affect voltage. These incentive signals for voltage management could be combined with energy prices that are obtained through a more traditional TE market to enable houses to manage loads and PV generation in a way that provides voltage control on the local feeder and frequency control on the bulk grid.

NREL used TOU prices as the energy price for their simulations, which they performed using the Integrated Energy Systems Model (IESM) co-simulation platform. The IESM was developed to evaluate the impacts of emerging consumer technologies under different tariff and market structures. It integrates the simulation of a distribution feeder, buildings (including thermal performance and building appliances), DERs including PV and battery systems, and controllers such as a home energy management system (HEMS). The IESM is designed to run in a high-performance computing system to allow the parallel execution of hundreds of instances of complex controllers - for example, HEMS algorithms based on a model predictive control approach. The IESM can also be interfaced with residential appliances and rooftop PV systems to perform hardware-in-the-loop simulations.

Simulation results show that the proposed TE control approach is very effective in reducing the number of voltage violations. The TE approach reduced accumulated voltage violations by approximately $80 \%$ compared to the simple TOU rate with the same weather scenario. The average air temperature change across all houses was less than $2^{\circ} \mathrm{F}\left(1^{\circ} \mathrm{C}\right)$, and thus the occupants' comfort was impacted only minimally. In addition, the number of capacitor bank and voltage regulator operations decreased by about $10 \%$ and $20 \%$, respectively.

The average house energy cost was unchanged compared to the TOU baseline; however, the TE control scenario has more outliers in energy cost, and a few houses have significantly higher or lower costs than the median cost. More extensive analysis and design are required to determine appropriate levels of compensation for homeowners while also considering revenue impacts for the utility. Future work is warranted to address how unique prices can be applied to each home while ensuring a socially acceptable level of fairness.

\subsubsection{Tata Consultancy Services (TCS)}

TCS used the TE Challenge as an opportunity to explore dynamic microgrid reconfiguration as a means to improve resiliency while maximizing use of renewables. TCS used a modified grid configuration with truncation of the network at three-phase nodes and inclusion of aggregate downstream single-phase loads and generators at each node. This was done in order to apply a dynamic microgrid algorithm that is designed to work with a three-phase network. Apart from this, TCS also developed an independent TE market model and used the GridLab-D simulation environment to validate the market model for the IEEE 8500 network scenarios established for the Challenge. The end goal remains to combine the TE market with the microgrids.

A dynamic microgrid configurator (DMC) was created that continuously monitors grid topology, load conditions and renewable generation at each instant to plan for segmented 
microgrid operations. Dynamic microgrids change the grid topology to create self-balanced regions that serve a maximum number of customers. The islanded microgrids also allow renewable generators to remain connected and producing power, when otherwise they would be disconnected when the distribution grid (without microgrids) has lost power. Thus, green energy utilization is maximized. The algorithm continuously monitors feeder loads and defines boundaries for interconnected virtual microgrids having a balanced generation and demand. When a fault occurs, the DMC can sectionalize the feeder into microgrids that are best able to maintain operation of the grid with minimum load curtailment. In practice, the feasibility of sectionalizing would depend on the circuit breakers and isolators available in a particular network segment.

TCS used a test sequence with four scenarios: base sunny, base cloudy, DMC with faults, and then the TE market approach independent of DMC. In the DMC simulation, three fault scenarios were defined to study the effectiveness of the DMC algorithm from a resiliency point of view. Line break faults were modeled at the substation, at the tie line connecting two microgrids, and within a microgrid. For each case, utilization of green power, reduction of losses, and percentage of load served were studied. Results indicate higher percentages of renewable power used and reduced losses with higher percentages of load served for operations. For the substation fault case, about $50 \%$ of the load could still be continuously served through reconfiguration, compared to momentary interruption of the entire circuit followed by partial restoration of less than $10 \%$ of total load in the case of conventional operation. For the tie line fault case, the microgrid reconfiguration allowed all load to remain supplied with power compared to losing power. In the third case of internal microgrid fault, there was no difference in result. For the three faults studied, the reconfigured topology ensured $100 \%$ utilization of available distributed generation.

\subsubsection{Massachusetts Institute of Technology (MIT)}

MIT simulation research was performed using their Scalable Electric Power System Simulator (SEPSS) [6]. MIT worked with a truncated version of the TE Challenge grid, aggregating single phase DER up to the three-phase nodes - all house loads and PV systems are included as if they were individually attached directly to the MV nodes. In the MIT TE approach, the distribution grid operator uses alternating current (AC) optimal power flow (OPF) to model voltage on the grid and then combines this with models of each DER that represent real and reactive power use/production as a function of voltage. Individual devices use model predictive control to estimate power usage and make bids for real and reactive power. Multi-rate simulations support a composite control design with a bidding layer at the slowest timescale and control adjustments mapped to real and reactive power adjustments at multiple time scales through the closed-loop control relations.

The MIT TEM approach seeks to maximize DER participation and minimize grid losses. This is accomplished through: relaxations on comfort requirements, use of batteries, and use of inverters for reactive power production. In addition, substation voltage is adjusted to minimize grid losses. Their static analysis (5.5.2) shows how different tariffs can result in increased profits or losses for grid entities, and how social welfare is impacted. Use of inverters to inject reactive power and of batteries to absorb PV-generated power during sunny times and produce power during times of high load (during the storm or in the evening) was able to reduce grid losses while increasing DER participation (increased comfort and use of PV power).

Their continuous simulation analysis (5.5.3) compares results of different TE Challenge scenarios, studying the ability of their control approach to maintain grid voltages while minimizing cost to customers. Their results show that grid voltages are always maintained 
within ANSI limits, no matter what tariff prices are used. However, they also show that the LMP-based dynamic price tariff, in combination with their control approach, results in the lowest cost to customers, with a $30 \%$ reduction in energy bills compared to their TOU tariffs. They also highlight some additional results. They note the impact of batteries to provide a nearly $20 \%$ average reduction in grid losses (Fig. MIT-15), with a greater than $20 \%$ reduction in utility-provided real power generation during the storm passing (Fig. MIT-13). They note the benefit of inverters to consume reactive power and reduce the flow of reactive power to utility generators by approximately $15 \%$ during the sunny periods of the day (Fig. MIT-14). They also note an interesting dynamic imbalance where the inverter-based reactive power injections compete with grid-side reactive controllers (Fig. MIT-12).

MIT provides a look at some factors that utilities and regulators need to consider in crafting tariffs. These include the impact of tariffs on consumer provision of real and reactive power, the impact of poorly designed TOU tariffs, the potential of PV with batteries to mitigate reverse power flows, the impact of TE on shunt reactor operation, the potential for oscillatory interactions between price-responsive loads and distribution grid voltage control actions, and the result of all these things on grid losses and cost to customers as well as utilities.

\subsubsection{Vanderbilt University}

Vanderbilt focused on the TE Challenge goal of TE co-simulation platform development. They developed a web-based generic co-simulation platform that can be customized and augmented to support a variety of heterogeneous simulations. The co-simulation platform, known as CPSWT-TE, is based on the widely-used IEEE distributed simulation standard called High-Level Architecture (HLA). HLA provides services that are critical to support distributed simulations: time-management, distributed object management, distributed simulation management, and support for real-time components. A user of the co-simulation platform can make use of existing library components as they build their own experiments adding their own specific components. Experiments run in the cloud.

The initial versions of both the CPSWT-TE and also a GridLAB-D design studio are now available for co-simulation use.

\subsection{Conclusions}

The TE Challenge was successful in many respects, with the following key accomplishments.

1. Developed a foundational TE co-simulation abstract component model. This model provides a common vocabulary for TE co-simulation and a foundation for interoperable TE simulations based on simulation components compatible with the abstract model.

2. Advanced co-simulation modeling tools and platforms that can support industry needs for TE system performance analysis.

3. Developed a reference common TE Scenario that can support ongoing comparative simulations.

4. Tested various TE approaches for integrating distribution grid DER while managing voltage. The results provide industry with understanding of the potential for TE methods to support DER integration.

The participating teams demonstrated the following.

- Volt-VAR control of inverters led to a $60 \%$ decrease in voltage violation duration. Volt-VAR inverter control provides better voltage control than HVAC temperature setpoint control and Volt-Watt controls. PNNL simulations demonstrate how such methods for voltage control might work on a real feeder with real residential devices. 
- Power flow modeling of grid with per-meter adjustment of prices for real and reactive power led to $80 \%$ reduction in voltage violation duration. Real and reactive TE markets can support both energy balance and distribution voltage control. NREL presents an approach to TE that uses frequent (every $10 \mathrm{~s}$ ) meter reads to perform AC optimal power flow of the grid to then formulate prices on an individual meter basis to drive device response to system voltages.

- Dynamic microgrid reconfiguration improves fault tolerance and balancing and enables use of renewable resources in fault conditions. TCS showed the potential for dynamic grid reconfiguration to properly distribute renewable power and maintain islanded microgrids during times when bulk grid power is not available.

- Energy bills for customers can be lowered with dynamic prices. MIT demonstrated this result, indicating increased system efficiency with value-based power delivery.

- As a corollary to the above, a dynamic TE tariff based on substation LMP is more effective than a TOU tariff for managing energy balance and voltage.

- TE can result in fewer control actions for distribution grid equipment, as found by NREL. PNNL demonstrated the positive impact of automatic Volt-VAR and VoltWatt controls to also reduce control actions. MIT saw decreased control actions except for an imbalance condition where inverter reactive power control would induce capacitor bank setting changes.

- Grid line losses with PV systems may be reduced by using TE, particularly when batteries are included to reduce reverse power flows, as shown by NREL and MIT.

- Control of substation voltage and grid equipment settings, combined with intelligent voltage-aware DER that can adjust settings to manage real and reactive power consumption or production, can together maintain voltage within ANSI limits while delivering acceptable comfort to customers (MIT result).

- Customer inverter reactive power provision can be useful for real voltage management and loss reduction, as demonstrated by PNNL, NREL, and MIT teams.

The results of different teams show in every case that a transactive approach improved grid operation, although with differences in: number of voltage violations, customer comfort, power flows and losses, count of control actuations, complexity of price computations and markets, frequency and number of sensor measurements, and social welfare (cost to customer and utility). The collection of research presented in this report provides utilities and regulators a clear idea of the potential of TE for grid management, as well as some understanding of technical requirements to implement one TE method or another. On the one hand, there may be opportunity to craft a successful TE market that supports energy balance with a single price for all feeder customers, with that price tied to LMP values. And customer DER may be independently managed to provide real and reactive power for voltage control. Alternatively, a distribution utility may use frequent meter reads and power flow modeling to get a more accurate picture of system voltages that can then be used to better manage voltage, even to the point of incentivizing loads and DER with prices tailored to each customer. This might be used to manage local PV-induced voltage rise or dip.

TE is still a new concept which needs further theoretical development, simulation testing of proposed solutions, and real-world demonstrations. The projects noted in Appendix A are a start, and more are ongoing as of the writing of this report. It is anticipated that work will continue as electric grid stakeholders work together to understand how best to integrate customer DER as we collectively move to a lower carbon grid of the future. 


\subsection{Overview References}

[1] GridWise Architecture Council, "GridWise Transactive Energy Framework Version $1.0, " 2015$.

[2] M. Burns, E. Song, D. Holmberg, "The Transactive Energy Abstract Component Model," NIST Spec. Pub 1900-602, 2018.

[3] D.G. Holmberg, S.T. Bushby, "NIST Transactive Energy Modeling and Simulation Challenge for the Smart Grid-Phase I Report," NIST Tech. Note 2019, 2018.

[4] D. Holmberg, M. Burns, S. Bushby, and A. Gopstein, "Transactive Energy Challenge Phase II Scenario," NIST Tech. Note 2021, 2018.

[5] D. Arnatt, "The IEEE 8500-node test feeder," in Transmission and Distribution Conference and Exposition, 2010.

[6] Ilic, M.D., Jaddivada, R., \& Miao, X., Scalable Electric Power System Simulator, Innovative Smart Grid Technologies-Europe (IEEE ISGT Europe), 2018. 


\title{
Section 2 PNNL Team Report: Transactive Energy System Simulation for DER Integration with Voltage Response
}

\author{
Tom McDermott, Yingying Tang, Qiuhua Huang \\ Pacific Northwest National Laboratory, Richland, WA
}

\begin{abstract}
In a supporting role, PNNL constructed the base case for all participants from an IEEE 8500node radial distribution feeder. A "feeder generator" was customized to populate this feeder backbone with houses, thermostat-controlled loads (air conditioning, water heaters), photovoltaic generation, and battery storage. Regulator tap changer and capacitor switching metrics were added to GridLAB-D, and the execution time of metrics collection was improved. The billing algorithm inside GridLAB-D was extended for time-of-day tiered rates, as defined by Tucson Electric Power.

In a research role, PNNL modified its double-auction transactive mechanisms to incorporate local autonomous voltage controls. Based on local meter voltage, a transactive agent can immediately modify its own responsive load or smart inverter functions, attempting to correct local voltage violations. Local autonomous control was needed because the voltage violations can occur much faster than the shortest market clearing interval we consider, which is five minutes.
\end{abstract}

\section{Table of Contents}

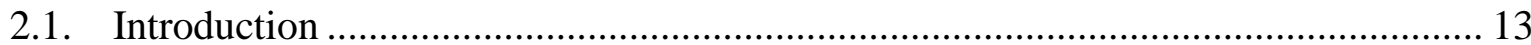

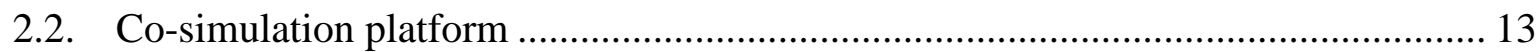

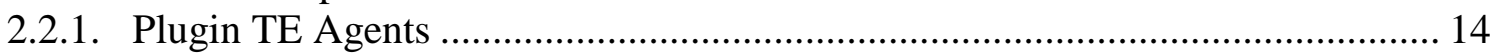

2.2.2. Messages between GridLAB-D and Controller Agents .................................. 14

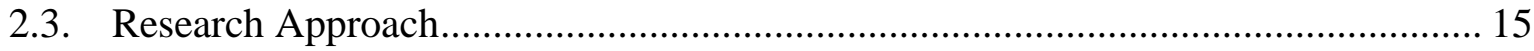

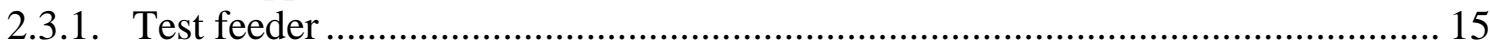

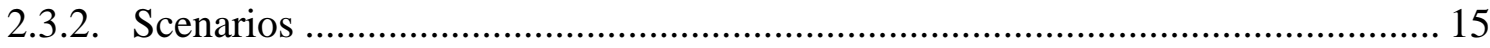

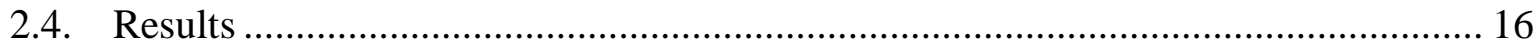

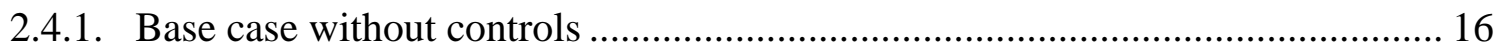

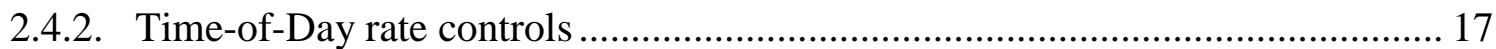

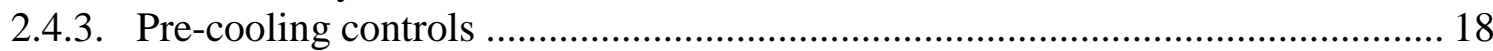

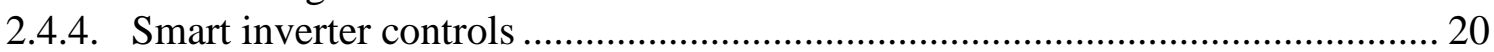

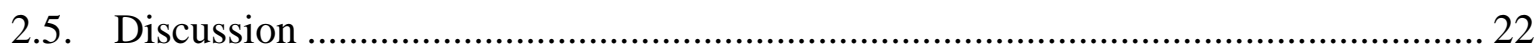

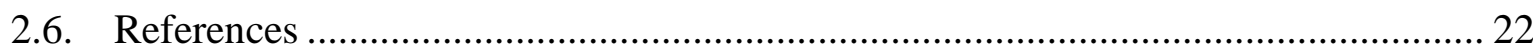




\subsection{Introduction}

The PNNL team includes developers of both GridLAB-D and the Transactive Energy Simulation Platform (TESP) [1]. Our goals include:

1. Encourage the use of transactive energy system simulation by a broader community of researchers.

2. Explore the adaptation of transactive systems, which have been focused on power and energy, to help manage voltage problems.

Our primary tool is GridLAB-D, with a co-simulation framework called Framework for Network Co-Simulation (FNCS) [2] and other federated simulators. FNCS provides a message bus and time synchronization between simulators, using a sockets library called ZeroMQ. In other projects, we federate bulk system simulators (e.g., MATPOWER, PYPOWER, AMES), the large-building simulator EnergyPlus, the communication system simulator ns3, and various transactive software agents with GridLAB-D over FNCS. In this project, the base case requires only GridLAB-D. For PNNL research in this project, we only federated transactive agents to GridLAB-D over FNCS.

\subsection{Co-simulation platform}

The co-simulation platform includes distribution system simulator GridLAB-D for distribution system infrastructure and residential customers, transactive agents, and the FNCS which manages the time step synchronization and message exchange among GridLab-D and the plugin agents. The architecture of the platform is shown in Figure PNNL-1.

In previous work, the PNNL team developed plugin APIs for users to develop transactive or non-transactive agents and plug them into the platform. Agent developers can work with a smaller code module written in the language of choice (current examples include $\mathrm{C}++$, Java and Python). Furthermore, developers can work in a shorter cycle of specification, design, implementation, testing and refinement. This has reduced the effort and broadened the participation in developing new agents, compared to our team's earlier practice of developing TE agents inside of a complex program like GridLAB-D. The integration of separate TE agents opens the door to future design and test of brand new TE mechanisms.

It should be noted that the co-simulation platform could be flexibly extended by interfacing other simulators (for example, bulk power system simulators and building simulators) with FNCS. A good example is the TESP.

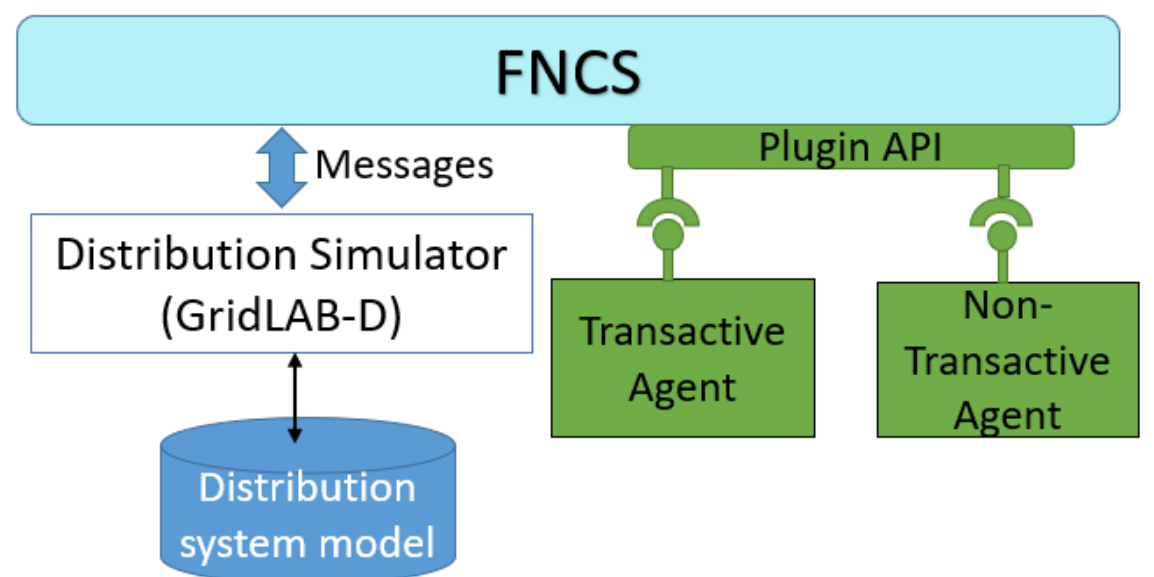

Figure PNNL-1 Architecture of the co-simulation platform for NIST TE Challenge II 


\subsubsection{Plugin TE Agents}

1) Double Auction TE Agent

A double-auction market is a two-way market, in which both suppliers and consumers (sellers and buyers) submit bids, including price and quantity, into a single energy market simultaneously [3]. The suppliers include distributed generation in the distribution system, as well as the bulk power supply from transmission systems. The demanders are the residential end-use loads. The double auction TE agent receives the supply and demand bids, resolves the bids into a common cleared market price and quantity, and delivers the cleared information (price and quantity) back to all participants.

\section{2) Thermostat Controller TE Agent}

A thermostat controller TE agent subscribes to the air temperature, HVAC power state, and the HVAC power if turned on from the associated house within GridLAB-D. The thermostat controller TE agent uses this information to help formulate a bid for electric power at the next market clearing, primarily the price and quantity. After each market clearing, the house thermostat controllers use that clearing price subscription, compared to their bid price, to adjust the HVAC thermostat set point. More details about its mechanism and implementation can be found in [3].

\subsubsection{Messages between GridLAB-D and Controller Agents}

Messages between GridLAB-D and the controller agents are illustrated in Figure PNNL-2. The double-auction TE agent associated with the substation uses the block time-of-use rate at the substation bus to represent a seller in the next market clearing interval. Each thermostat Controller TE Agent subscribes to the air temperature, HVAC power state, and the HVAC power from the associated house within GridLAB-D. The controller uses this information to help formulate a bid for electric power at the next market clearing, primarily the price and quantity. Note that each market clearing interval will have its own market bid, and that rebidding may be allowed until that particular market bid closes. If accepted, a rebid simply supersedes the older bid for that market interval. When bidding closes for a market interval, the double-auction market will settle all bids and publish several values, including the clearing price, clearing quantity, average clearing price and its deviation. The house thermostat controllers use that clearing price subscription, compared to their bid price, to adjust the HVAC thermostat set point.

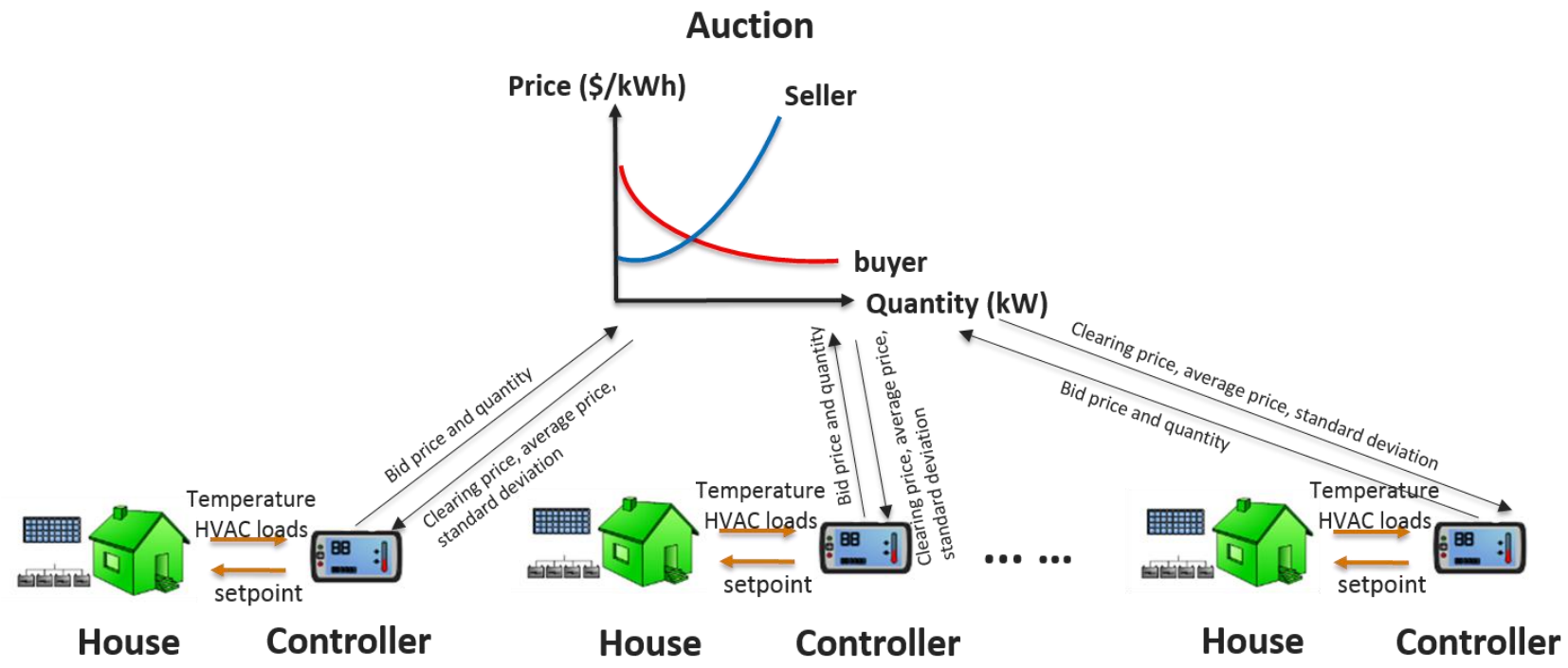

Figure PNNL-2 Transactive market topology 


\subsection{Research Approach}

\subsubsection{Test feeder}

In this project, PNNL has chosen a modified IEEE 8500-node feeder as the test feeder. The 8500-node test feeder represents a large radial distribution feeder, which has been designed to present challenges to distribution system analyses, such as voltage and VAR control simulations [4]. The feeder contains one voltage regulator controlling feeder voltage at the substation, and three other voltage regulators along the line. The feeder also contains four capacitors, three of them are controlled by voltage seen at the capacitor, and the other one is manually controlled. The placement of regulators and capacitors is shown in Figure PNNL-3.

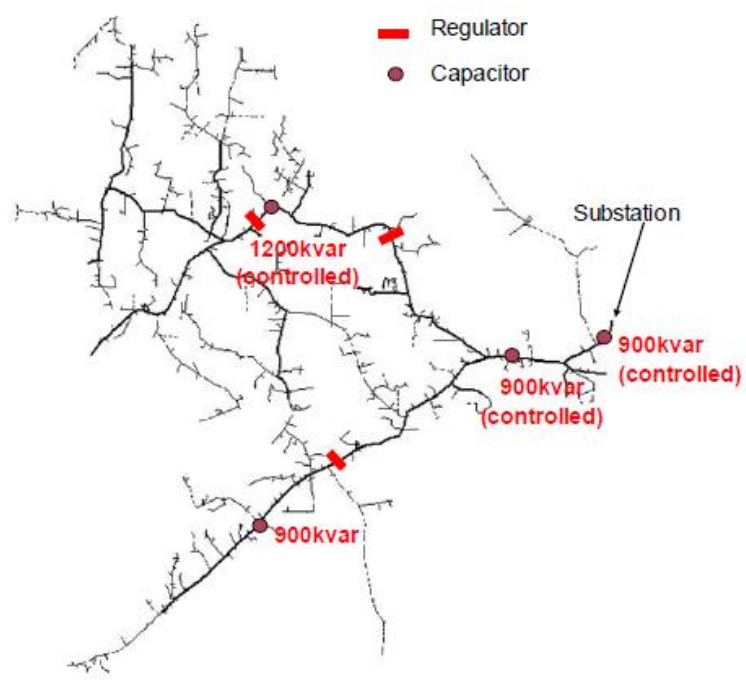

Figure PNNL-3 IEEE 8500-node test feeder diagram [4]

The test feeder was customized to conduct transactive analysis. House models were placed at the secondary side of the transformers. Each house contains one air conditioner and aggregated unresponsive ZIP loads. Water heater and solar inverters are also included in some of the houses. A "feeder generator" was developed to populate the modified feeder model in GridLAB-D. Table PPNL-1 lists the main properties of the modified test feeder.

Table PPNL-1 Customized IEEE 8500-node feeder properties

\begin{tabular}{|l|l|}
\hline Nominal peak load & $10773.2+\mathrm{j} 2700.0 \mathrm{kVA}$ \\
\hline Houses & $\begin{array}{l}1977 \text { houses ranging from } 500 \text { to } 3500 \mathrm{sf}\left(46.5 \text { to } 325 \mathrm{~m}^{2}\right), \text { total } \\
\text { area } 3941782 \mathrm{sf}\left(366,200 \mathrm{~m}^{2}\right)\end{array}$ \\
\hline Electric water heaters & 1013 electric water heaters, of total ratings $4574.7 \mathrm{~kW}$ \\
\hline Air conditioners & 1977 air conditioners, of total ratings $26150.6 \mathrm{~kW}$ \\
\hline Solar & 1777 solar inverters, of total ratings $6755.2 \mathrm{~kW}$ \\
\hline Non-responsive ZIP load & $\begin{array}{l}\text { All non-responsive ZIP loads are constant current loads, with } \\
\text { power factor } 0.95\end{array}$ \\
\hline
\end{tabular}

\subsubsection{Scenarios}

In order to investigate impacts of different approaches on reducing voltage violations, several scenarios were proposed and implemented. All the cases used the customized IEEE 8500node test feeder model in GridLAB-D. The simulation duration is one day with 15-minute time intervals. The weather data, including clear-day and cloudy-day data, have been generated by processing typical meteorological year (TMY) files in MATLAB to produce test files, with coordinated temperature and irradiance variations. The billing algorithm inside 
GridLAB-D was extended for time-of-day tiered rates, as defined by Tucson Electric Power [5].

Graphical results and tabular summaries can be generated from the simulation. The primary metrics of interest include:

1. House air temperature (minimum, maximum, and deviation from set point)

2. Capacitor switching and regulator tap changing counts

3. Voltage violation counts and durations

4. Total solar power production

5. Count of smart inverter mode changes

The base case is conducted by running the simulations without transactive agents or other controls. The voltage violations in the base case are compared with other cases which adopted time-of-day tiered rates, or local autonomous controls such as pre-cooling agents and smart inverter functions. The results have revealed that smart inverter Volt-VAR function has the most significant impact on reducing occurrence of voltage violations.

\subsection{Results}

\subsubsection{Base case without controls}

In the base case scenario, with fixed price tariff and including the storm front, each house has been assigned a randomized thermostat setting schedule, which represents the residential temperature adjustments over the day. The simulation has been conducted without any controls. Figure PNNL-4 shows the graphical results of the primary metrics.
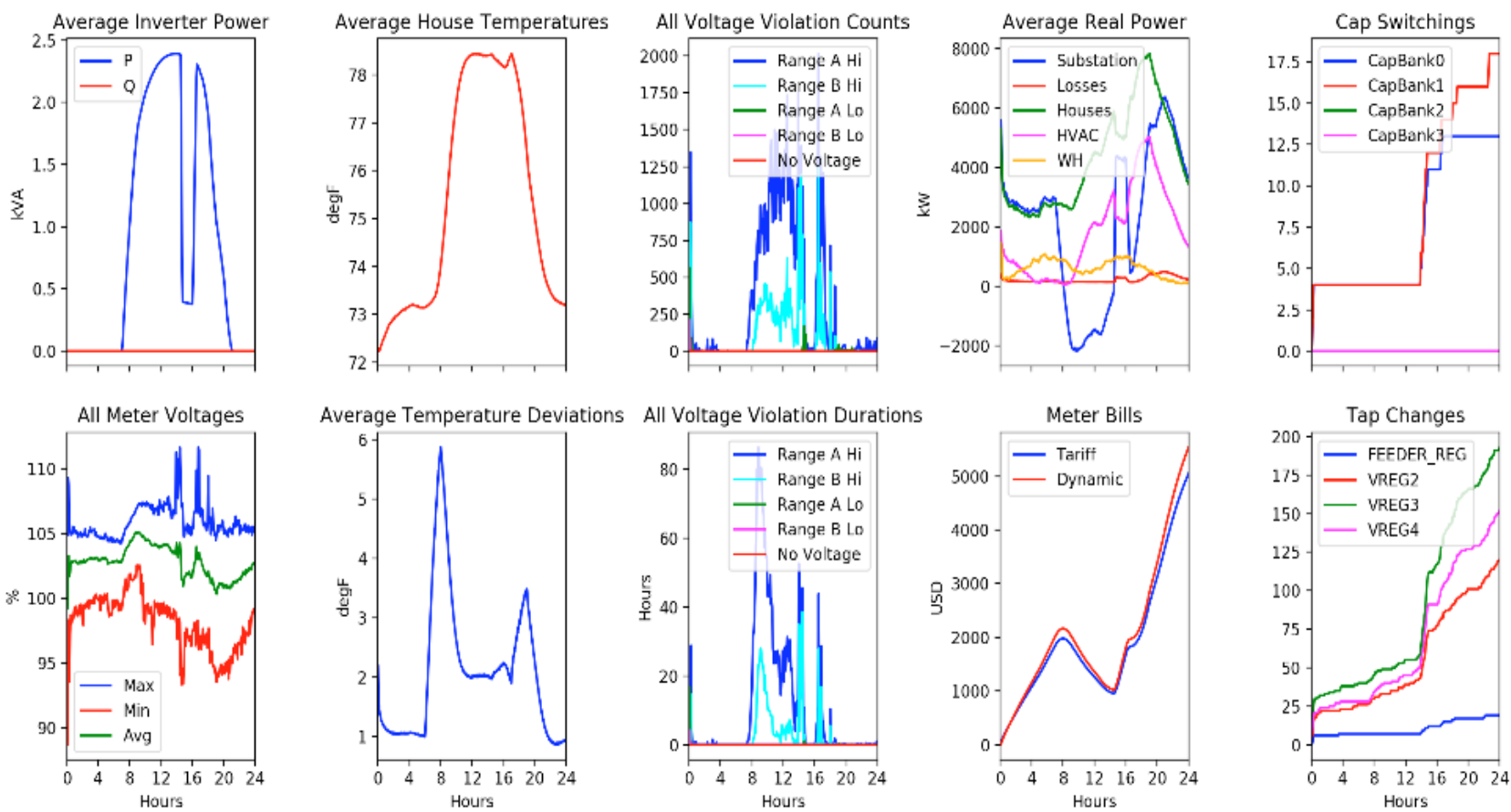

Figure PNNL-4 Base case simulation results

As summarized from Figure PNNL-4, the highest average house temperature is $78.5 \mathrm{~F}$, happening around 12:00 PM. By summing up the duration of voltage violations of all houses in the whole day, there are $797 \mathrm{~h}$ in total that violate $\mathrm{B}$ range voltage. There are 23 capacitor switching operations, and 388 tap changes. 


\subsubsection{Time-of-Day rate controls}

In the second scenario, time-of-day price tariff, in order to investigate market impacts on voltage, each house set point is controlled by a transactive ramp controller in the cosimulation platform. Figure PNNL-5 provides an example of cooling set points of the transactive controller, with parameters explained in Table PNNL-2. With the cleared market price, the adjusted set point can be calculated by:

$$
T_{\text {adjusted }}=T_{\text {base }}+\frac{\left(P_{\text {cleared }}-P_{\text {average }}\right) *\left|T_{\max / \min }\right|}{k_{\text {high } / \text { low }} * \sigma}
$$

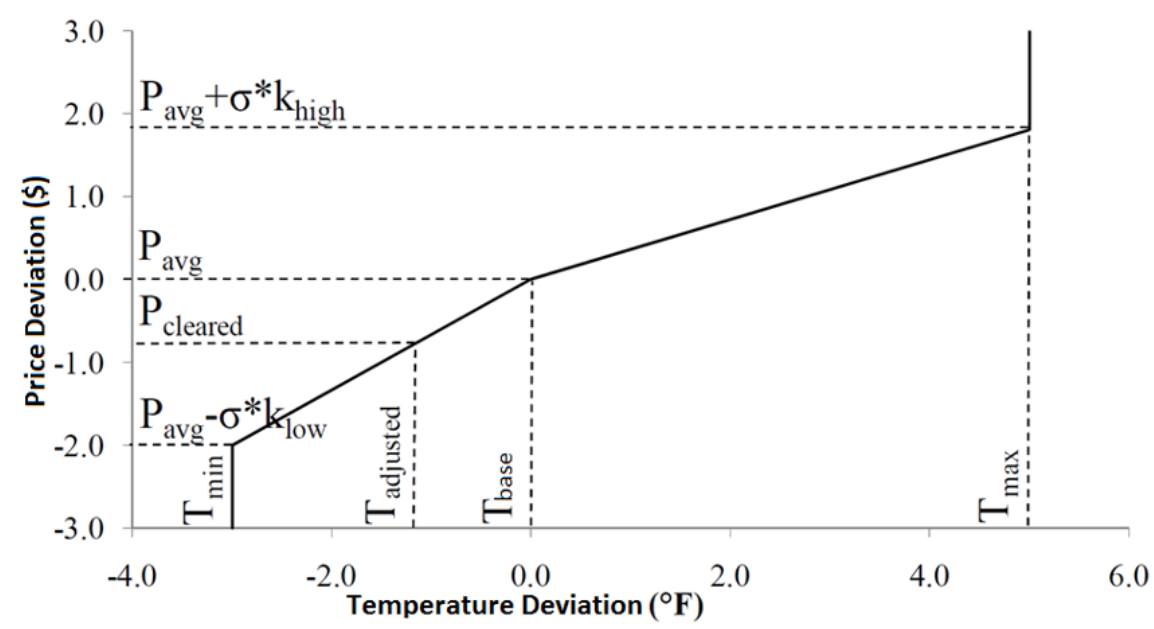

Figure PNNL-5 Bid and response of transactive ramp controller

Table PNNL-2 Parameters in controller bid and response curve

\begin{tabular}{|l|l|}
\hline$P_{\text {avg }}$ & Average market cleared price \\
\hline$P_{\text {cleared }}$ & Market cleared price in current market period \\
\hline$T_{\min }$ & The temperature set point limit on the low side \\
\hline$T_{\max }$ & The temperature set point limit on the high side \\
\hline$T_{\text {base }}$ & Base temperature set point \\
\hline$\sigma$ & Standard deviation of market cleared price \\
\hline$k_{\text {high }}$ & The comfort response above the base set point \\
\hline$k_{\text {low }}$ & The comfort response below the base set point \\
\hline$T_{\text {adjusted }}$ & Adjusted set point based on cleared market price \\
\hline
\end{tabular}

A time-of-day rate, as seen in Figure PNNL-6, is applied to the controller. With average market price $P_{\text {avg }}$ of $0.1167 \$ / \mathrm{kWh}$, and standard deviation $\sigma$ of $0.0149 \$ / \mathrm{kWh}$, the time-ofday rate is applied on Figure PNNL-5 as cleared market price ( $\left.\mathrm{P}_{\text {cleared }}\right)$, to obtain the adjusted set point at every market cycle. 


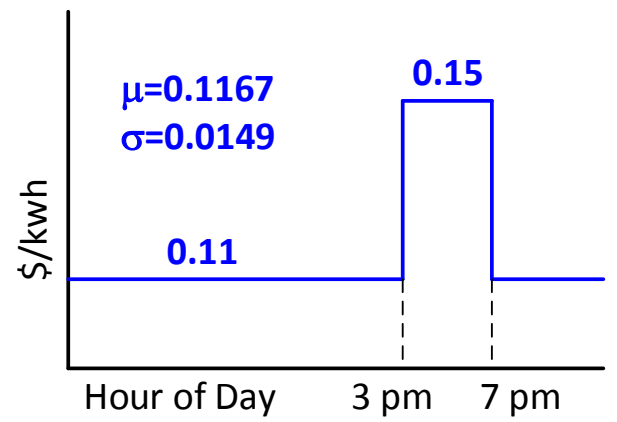

Figure PNNL-6 Time-of-day rate applied to controller

Simulation results for this scenario are shown in Figure PNNL-7. The time-of-day rate has reduced $\mathrm{B}$ range voltage violation duration to a total of $574 \mathrm{~h}$, compared to the base case of $797 \mathrm{~h}$. There are 16 capacitor switching operations, and 401 tap changes.
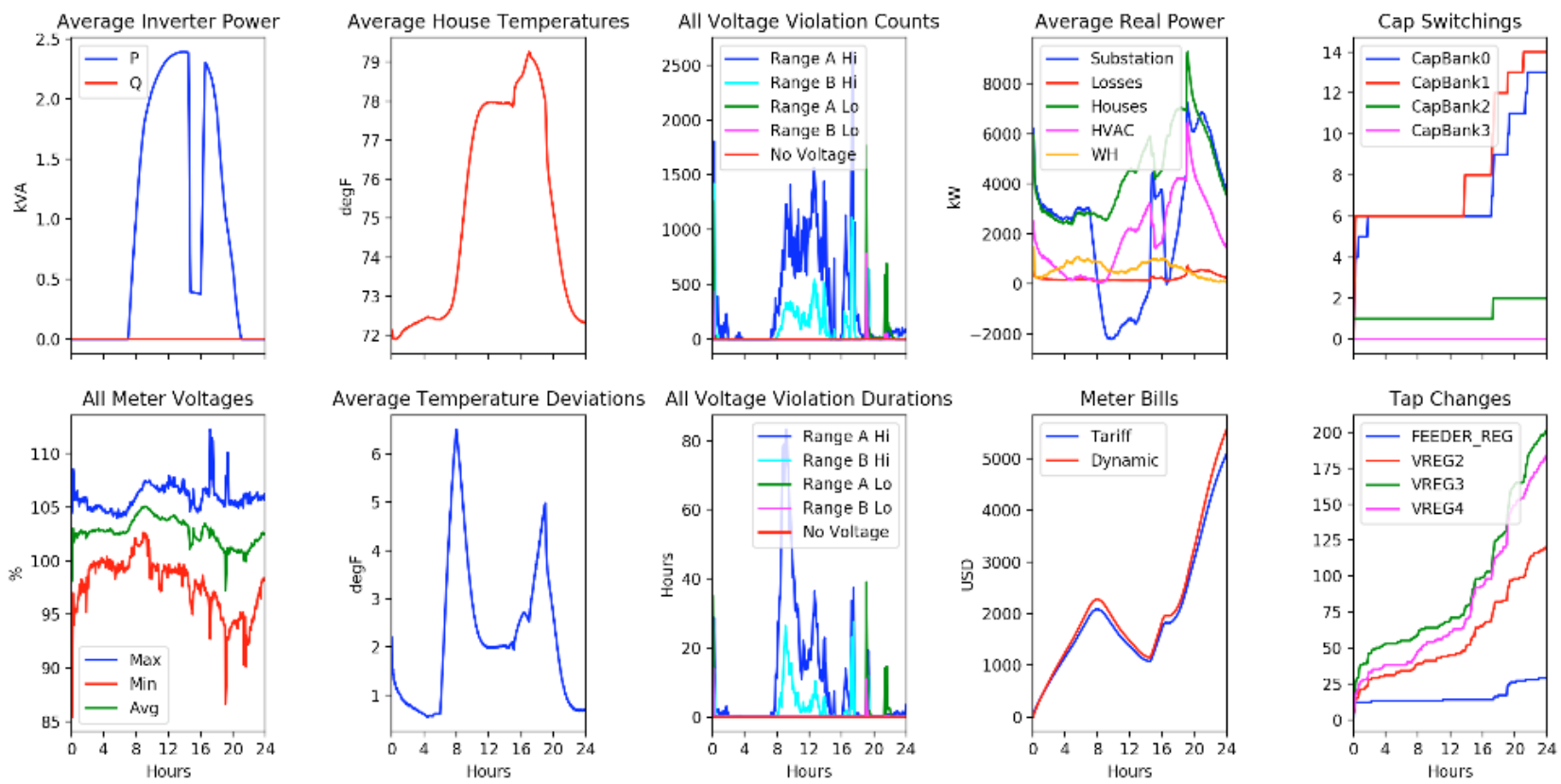

Figure PNNL-7 Scenario 2 simulation results

\subsubsection{Pre-cooling controls}

In Scenarios 3 and 4, voltage violation reductions are realized by controlling the secondary voltage. As illustrated in Figure PNNL-8, by increasing energy consumption from houses and reducing real power output from residential PV systems, the over-voltage violation issues can be improved. Controlling reactive power outputs of the residential PV systems can also help improve voltages. 


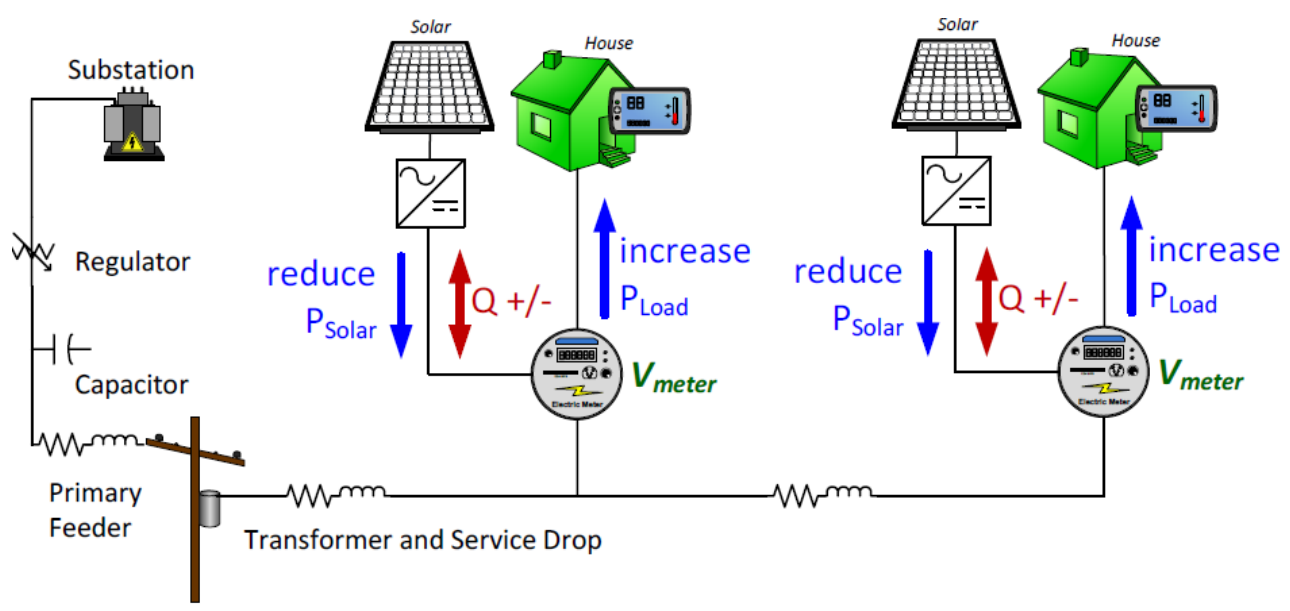

Figure PNNL-8 Voltage control at secondary side

Figure PNNL-9 shows the effect of voltage change with different real and reactive power injections from a PV system in the test feeder. This case corresponds to a case with 7-kW solar generation connected to the secondary of a single-phase 25-kVA transformer. The total impedance seen by the inverter is $0.0318+\mathrm{j} 0.02082 \Omega$ at $120 \mathrm{~V}$. The impact of reactive power injection on the voltage change is smaller than that of real power injection, due to the fact that the equivalent resistance is larger than reactance at the transformer secondary side in this example. This sensitivity analysis result also indicates the feasibility of voltage control through pre-cooling control.

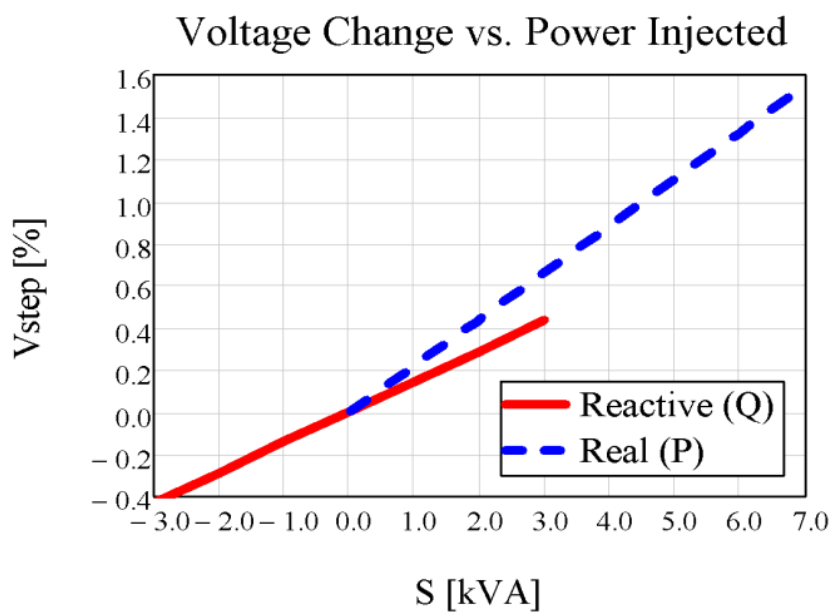

Figure PNNL-9 Relation between voltage change and power injection from the PV

In Scenario 3, a pre-cooling agent at each house is used to increase house energy consumption at the time when voltage is above threshold. The pre-cooling agent monitors the house voltage, and whenever the voltage is above the defined threshold $(125 \mathrm{~V})$, the current house set point will be applied with an offset of $4 \mathrm{~F}$ and will be locked in all day to avoid oscillations. In this way, the agent precools the house and thus increases the house load to reduce voltage violations. The pre-cooling agent, written in Python, has been implemented into the co-simulation platform.

Simulation results of Scenario 3 are shown in Figure PNNL-10. The B range voltage violation duration is $464 \mathrm{~h}$, which is less than that of Scenario $2(574 \mathrm{~h})$ using time-of-day rate. There are 22 capacitor switching operations, and 460 tap changes. 

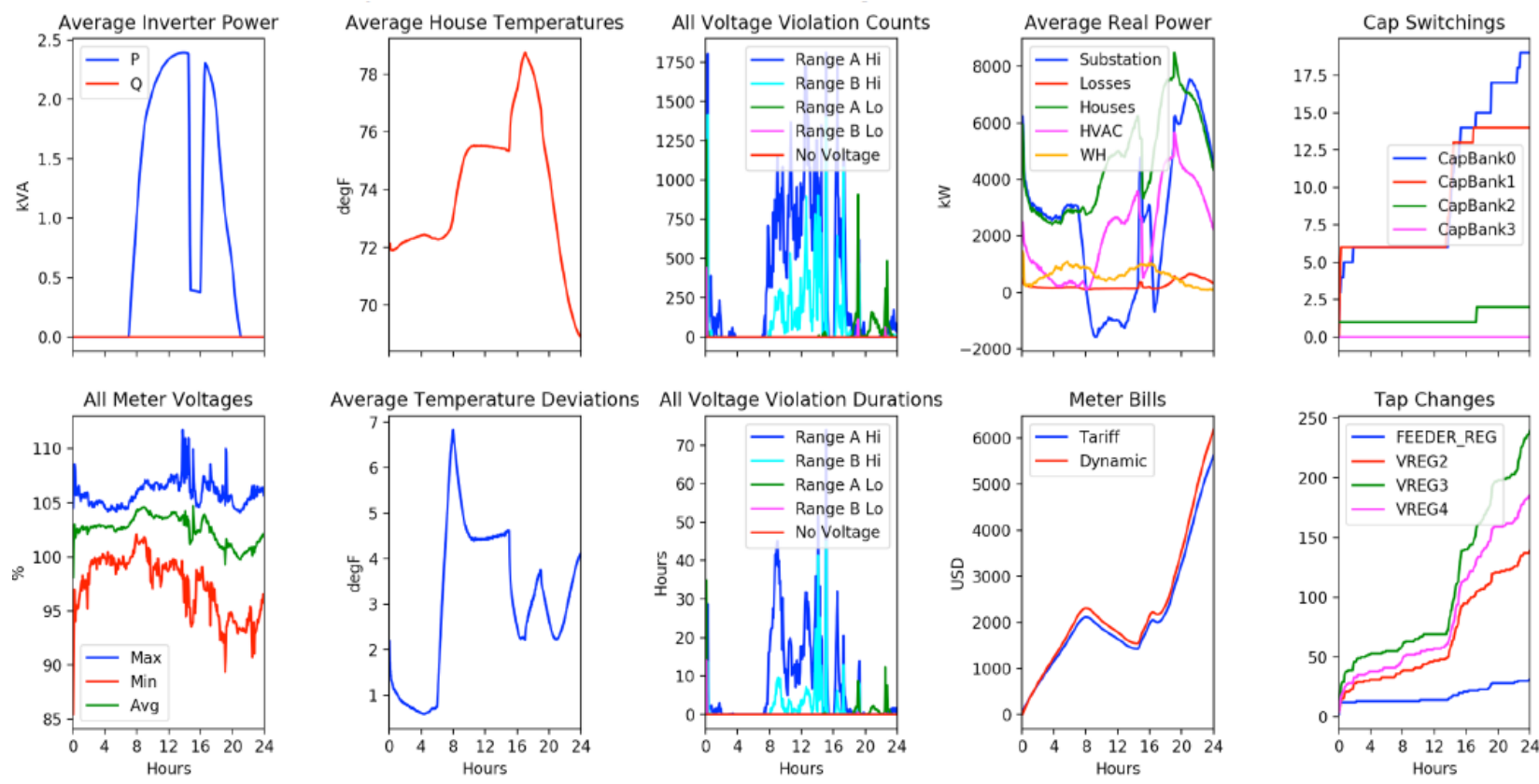

Figure PNNL-10 Scenario 3 simulation results

\subsubsection{Smart inverter controls}

In Scenario 4, smart inverter functions, including Volt-VAR and Volt-Watt, are applied to residential PV systems separately to improve voltage problems. Figure PNNL-11 shows the defined curves of Volt-VAR and Volt-Watt. When using Volt-VAR functions, as voltage at the PV inverter gets lower than 0.98 p.u., the inverter starts to inject reactive power. As voltage exceeds 1.02 p.u., the inverter begins to absorb reactive power. When using VoltWatt functions, the real power injection decreases as the inverter terminal voltage becomes higher than 1.05 p.u, and the injection reduces to zero when inverter terminal voltage is higher than $1.1 \mathrm{pu}$.
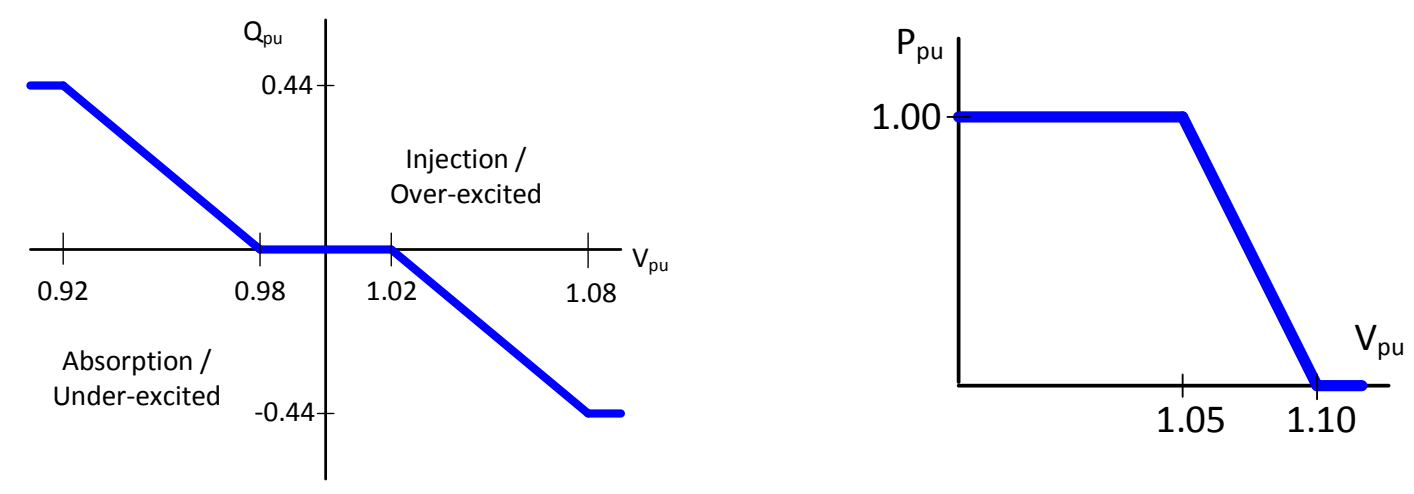

Note: $Q$ is given priority over $P$

Figure PNNL-11 Applied Volt-VAR (left) and Volt-Watt (right) functions; these are the defaults for category B ("high penetration") in IEEE 1547-2018.

Figure PNNL-12 shows the simulation results with Volt-VAR mode adopted. There are a total of $233 \mathrm{~h}$ violating B range voltage. In a one-day simulation, there are 5 capacitor switching operations, and 222 tap changes. Since Volt-VAR mode controls reactive power outputs, it has no impact on solar production, as seen from Figure PNNL-12. 

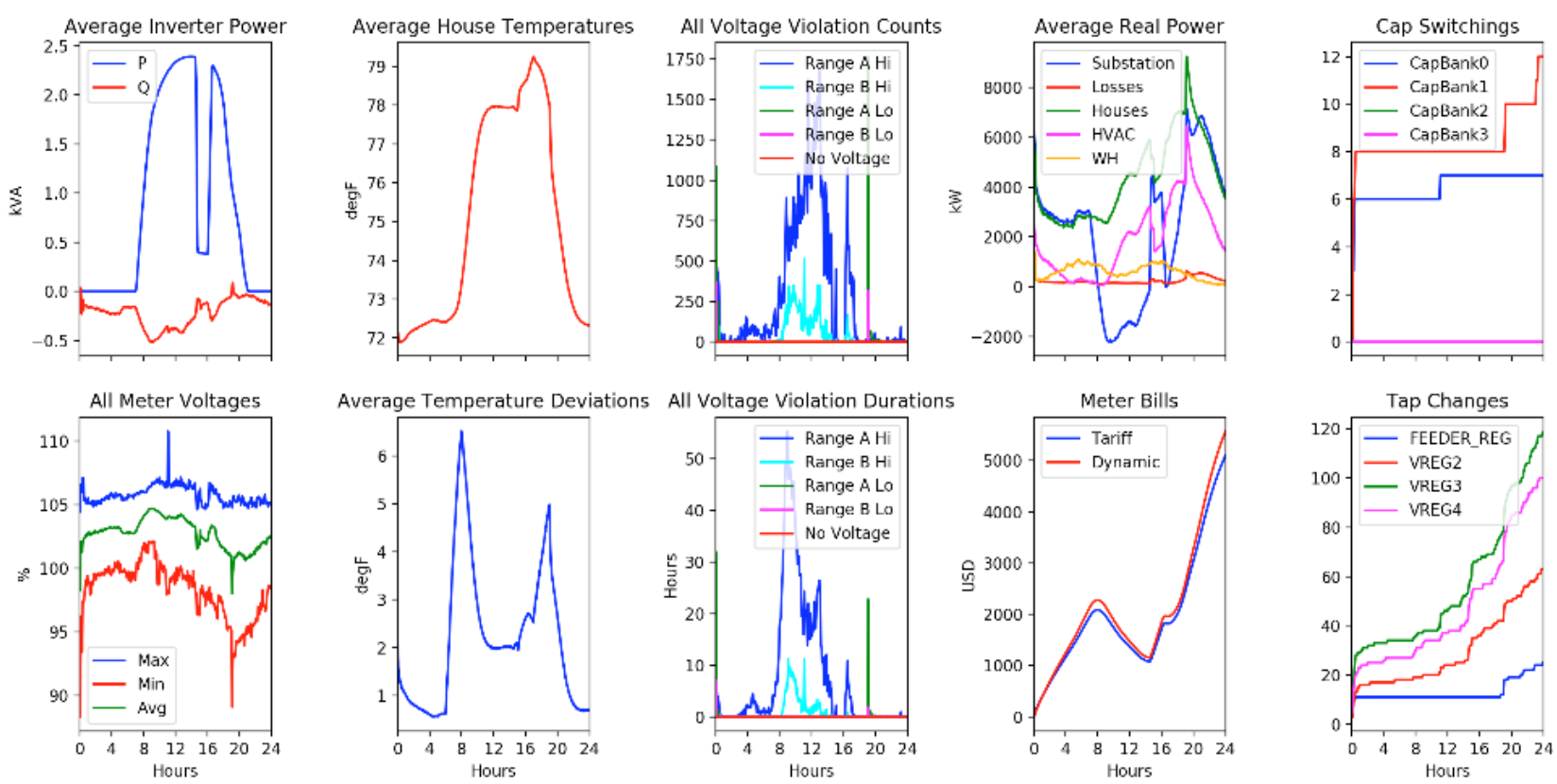

Figure PNNL-12 Scenario 4 Volt-VAR mode simulation results

Figure PNNL-13 shows the simulation results with Volt-Watt mode adopted. There are a total of $553 \mathrm{~h}$ violating B range voltages. In a one-day simulation, there are 17 capacitorswitching operations, and 394 tap changes. To reduce voltage violations, the Volt-Watt mode has decreased real power injections from PV inverters, resulting in a $0.75 \%$ reduction in solar production.
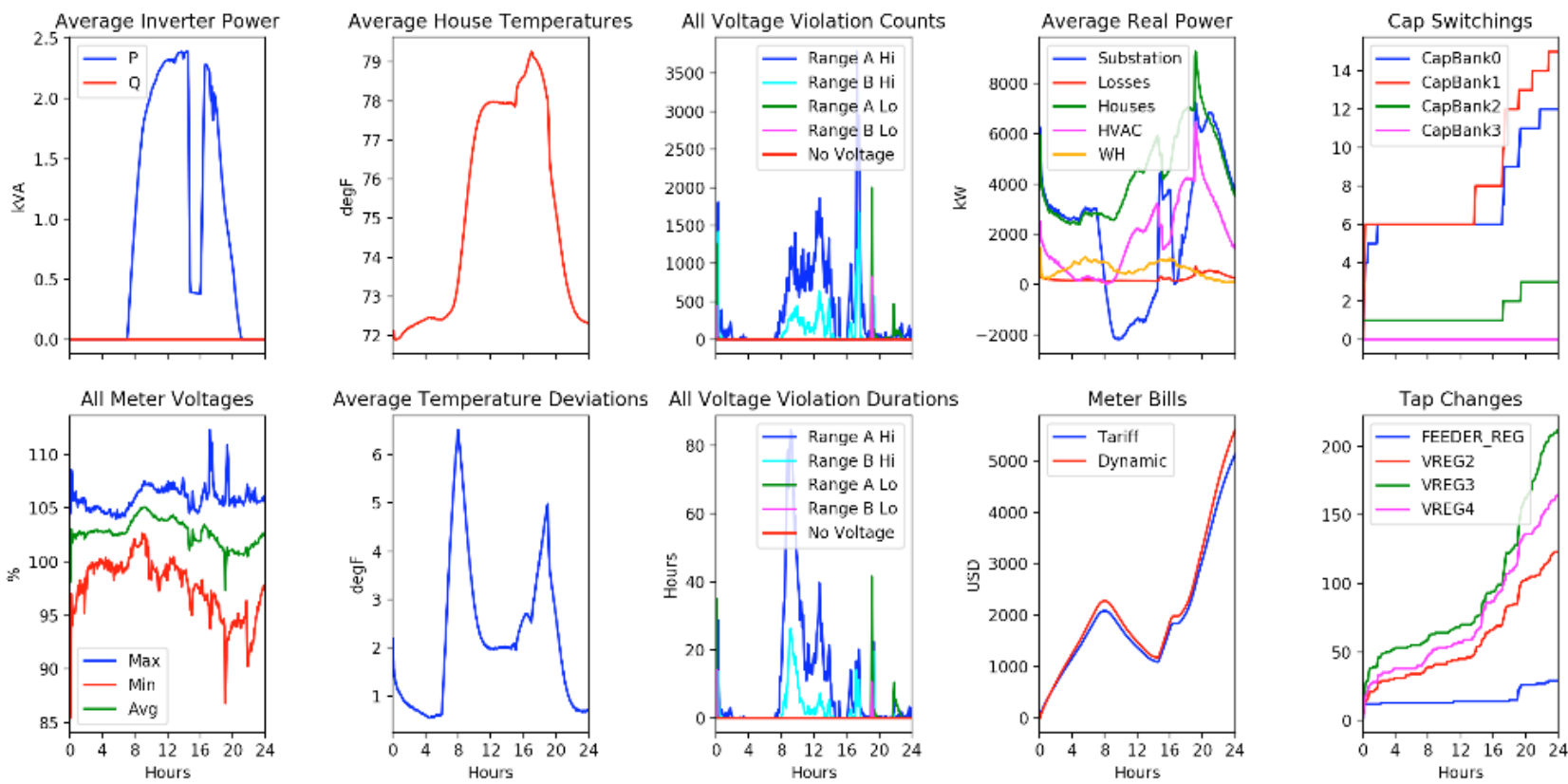

Figure PNNL-13 Scenario 4 Volt-Watt mode simulation results

Comparing Volt-VAR and Volt-Watt modes, Volt-VAR mode can be more aggressive and persistent and can involve more inverters controlling voltage violations. 


\subsection{Discussion}

The use case chosen for the TE Challenge focuses on voltage problems ${ }^{1}$, which can occur much faster than any transactive system's market clearing interval. For this reason, we chose to look at local autonomous controls in the context of a transactive system. The pre-cooling agent has a beneficial impact on overvoltage, but smart inverter functions are more effective system-wide. Especially in Volt-VAR mode, the inverters can participate more gradually at many locations before a voltage violation occurs. The pre-cooler could still provide value as a local protection for overvoltage, especially if there were no local solar generation to curtail.

The pre-cooler needs a voltage measurement at the meter, which is not normally available to smart thermostats and would be an extra cost. That cost would not be justified for the precooler use case only. However, there may be additional value in forecasting or control if voltage measurements were available to the transactive agent. This is an area suggested for further investigation.

In other transactive simulations, we have encountered stability issues with load and price due to the "cobweb effect" $[6,7]$. This project used a non-responsive tariff, so we didn't see the "cobweb effect", but we did experience voltage instability due to the two-step nature of our pre-cooler, until we implemented a simple lockout period. This example shows the value of system simulation (or system-of-system simulation) in designing and testing transactive agents so that they can work properly in the field, i.e., without unintended side or ill effects.

The typical meteorological year data is not intended to simulate weather events, so we had to create one for this project. Two CSV files of temperature, pressure, humidity, wind speed, direct solar irradiance and diffuse solar irradiance were created, one for the sunny day and one for the cloudy day (storm front). The cloud disturbance has a ramp, and there is a correlation between air temperature and solar irradiance. These files were created by processing a local TMY file through a MATLAB script, incorporating simple assumptions. Motivated by this experience, we have planned that a future version of TESP will have the option of using actual event CSV files obtained from a weather service.

\subsection{References}

[1] PNNL. (2017). Transactive energy simulation platform (TESP). Available: https://tesp.readthedocs.io/en/latest/

[2] S. Ciraci, J. Daily, J. Fuller, A. Fisher, L. Marinovici, and K. Agarwal, "FNCS: A framework for power system and communication networks co-simulation," in Symposium on Theory of Modeling and Simulation, San Diego, CA USA, 2014: ACM.

[3] J. C. Fuller, K. P. Schneider, D. Chassin, "Analysis of Residential Demand Response and Double-Auction Markets," in Proceedings of IEEE PES General Meeting, 2011.

[4] R. F. Arritt and R. C. Dugan, "The IEEE 8500-node test feeder," IEEE PES T\&D 2010, New Orleans, LA, USA, 2010, pp. 1-6.

[5] Tucson Electric Power, Pricing plans, www.tep.com/rates

[6] A. G. Thomas and L. Tesfatsion, "Braided cobwebs: Cautionary tales for dynamic retail pricing in end-to-end power systems," Economics Working Papers, pp. 1-19, July 30, 2017. Available: http://lib.dr.iastate.edu/econ_workingpapers/30.

[7] L. Zhao and W. Zhang, "Stability analysis of multi-period electricity market with heterogenous dynamic assets," arXiv, pp. 1-9, April 1, 2017. Available: https://arxiv.org/abs/1703.10267

\footnotetext{
${ }^{1}$ At the feeder levels, voltage transients can be on the order of seconds, which is much smaller than the typical 5-min market clearing interval.
} 


\title{
Section 3 NREL Team Report: Network-Aware Transactive Energy Control Simulations
}

\author{
Annabelle Pratt, Mark Ruth, Fei Ding \\ National Renewable Energy Laboratory, Golden, CO \\ Additional team members: Sivasathya Balamurugan ${ }^{1}$, Emiliano Dall'Anese ${ }^{1,2}$, Xinyang Zhou ${ }^{1}$, \\ Dheepak Krishnamurthy ${ }^{1}$, Rune Junker ${ }^{3}$, Prateek Munankarmi ${ }^{4}$ \\ (1) National Renewable Energy Laboratory, Golden, CO \\ (2) University of Colorado, Boulder, CO \\ (3) Technical University of Denmark (DTU) \\ (4) South Dakota State University, Brookings, SD
}

\begin{abstract}
NREL performed their simulation using the Integrated Energy Systems Model (IESM) cosimulation platform. They implemented network-aware TE controls in the IESM co-simulation framework that manages distribution feeder voltages based on real-time optimal power flow. This is one part of a multi-timescale TE control approach that NREL is developing to reduce costs for both balancing power supply and demand and managing distribution feeder voltages. This multi-timescale TE approach uses a price signal based on two components: an energy price based on wholesale prices and bids by participating DERs that adjusts on a 5-15 min market cycle, plus an incentive signal overlay for fast grid services, updated every 1-10 s.

For the TE Challenge, NREL simulated only the calculation of the incentive signals for voltage regulation services and used the time-of-use (TOU) price as the energy price. A distribution network controller monitors voltage at each house, estimates future power usage and flexibility of homes, runs a power flow model, and uses this information to generate per-house incentive signals for both active and reactive power. Collectively, the TOU energy price plus the per-house incentive signal enables the controllable house devices (air conditioners and PV) to provide voltage regulation services in addition to managing energy costs and comfort for house occupants.
\end{abstract}

The team found that PV system response to active and reactive power incentive signals and air conditioner response to active power incentive signals provided an effective tool to reduce voltage violations in addition to the load shifting produced in response to the TOU price. The TE approach reduced voltage violations by approximately $80 \%$ as compared to the scenario with the same weather and no control while holding the average air temperature to within $2{ }^{\circ} \mathrm{F}$ of the desired set point (indicating only a small impact on occupant comfort).

Analysis of the TE approach, as compared to the case of no control, showed nearly the same average energy cost but with wider cost variation, and a few houses with costs significantly higher or lower than the median cost. More extensive analysis using a longer test period is required to determine appropriate levels of compensation for PV curtailment and reactive power support that will ensure fair compensation to houses that provide voltage regulation services. Future work is also warranted to address how unique per-house prices can be applied while ensuring a socially acceptable level of fairness. 


\section{Table of Contents}

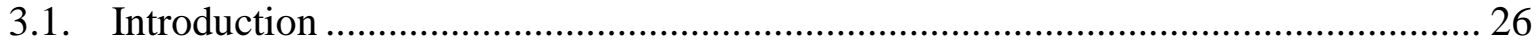

3.2. Co-Simulation Platform: Design and Interfaces Between Tools ............................ 27

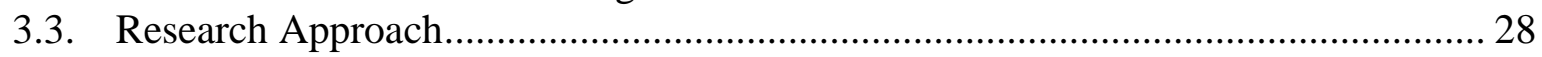

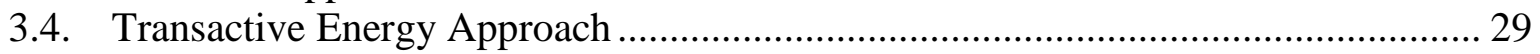

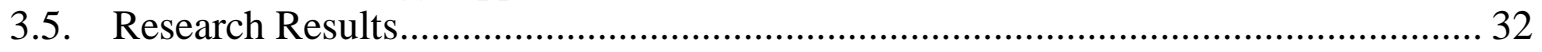

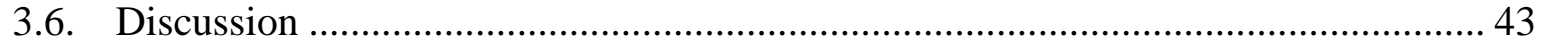

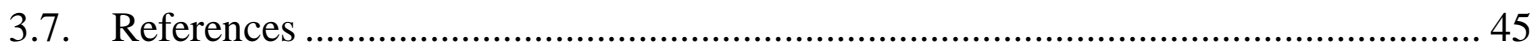

\section{List of Acronyms}

\begin{tabular}{ll} 
A/C & air conditioner \\
BESS & battery energy storage system \\
DER & distributed energy resource \\
DERMS & distributed energy resource management system \\
DLMP & distributed locational marginal price \\
DMS & distribution management system \\
DOE & U.S. Department of Energy \\
DSO & distribution system operator \\
DTU & Technical University of Denmark \\
ESIF & Energy Systems Integration Facility \\
EV & electric vehicle \\
FLOPS & floating point operations per second \\
HEMS & home energy management system \\
HPC & high-performance computing \\
IESM & Integrated Energy System Model \\
MPC & model predictive control \\
NIST & National Institute of Standards and Technology \\
NREL & National Renewable Energy Laboratory \\
PNNL & Pacific Northwest National Laboratory \\
PRD & price-responsive demand \\
p.u. & per unit \\
PV & photovoltaic \\
PSEC & Power Systems Engineering Center \\
SEAC & Strategic Energy Analysis Center \\
TE & transactive energy \\
TEC & transactive energy control \\
TOU & time of use \\
ZMQ & ZeroMQ \\
\hline &
\end{tabular}




\section{List of Figures}

Figure NREL-1. Diagram of the IESM including a co-simulation coordinator, simulated distribution feeder, simulated homes with home energy management systems, and markets..... 27 Figure NREL-2 Mapping of IESM components to the abstract component model developed by NIST

Figure NREL-3 Overview of multi-timescale TE controls approach ................................... 30

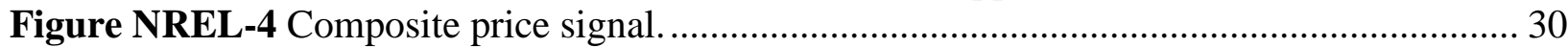

Figure NREL-5 Weather data used for simulations. ....................................................... 32

Figure NREL-6 The TOU rate used for simulations.................................................... 33

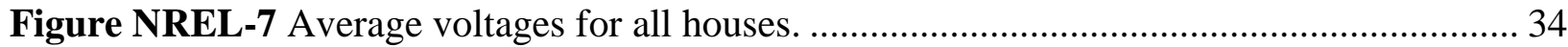

Figure NREL-8 Voltages at all houses for all four scenarios............................................ 34

Figure NREL-9 Proportion of houses with overvoltage violations..................................... 35

Figure NREL-10 Proportion of houses with undervoltage violations.................................... 36

Figure NREL-11 Cumulative number of voltage violations................................................. 36

Figure NREL-12 Incentive prices for active power generated by the network-level control.... 37

Figure NREL-13 Incentive prices for reactive power generated by the network-level control. . 37

Figure NREL-14 Average active (left) and reactive (right) net power consumed by houses..... 38

Figure NREL-15 Average active (left) and reactive (right) power output of PV inverters. ....... 38

Figure NREL-16 Average house air temperatures and average desired setpoint..................... 39

Figure NREL-17 Average power consumed by air conditioners. ......................................... 39

Figure NREL-18 Cumulative average energy cost for houses........................................... 41

Figure NREL-19 Cumulative average energy cost components for the TEC scenario.............. 41

Figure NREL-20 Averages and ranges of energy costs for houses. The TEC scenario has four additional outliers at $\$ 20, \$ 12.76, \$-9.56$, and $\$-27.22$ that are not shown............................. 42

Figure NREL-21 Number of capacitor operations (left) and number of voltage regulator tap

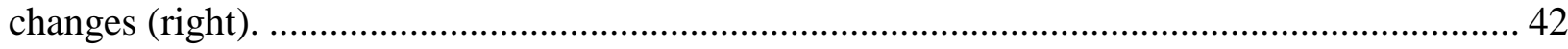

Figure NREL-22 Substation real (left) and reactive (right) power. ..................................... 43 


\subsection{Introduction}

The National Renewable Energy Laboratory (NREL) is the only federal laboratory dedicated to the research, development, commercialization, and deployment of renewable energy and energy efficiency technologies. NREL's mission is to advance the science and engineering of energy efficiency, sustainable transportation, and renewable power technologies, and to provide the knowledge to integrate and optimize energy systems. NREL's energy-analysis program informs policy and investment decisions as energy-efficient and renewable energy technologies advance from concept to commercial application to market penetration. Using objective technologyneutral analysis, NREL aims to increase the understanding of energy policies, markets, resources, technologies, and infrastructure, as well as the connections between these and economic, environmental, and security priorities.

The NREL team's goals for participating in the TE Challenge are twofold. The first is to make a key contribution to the field of transactive energy control using advanced distributed control approaches. NREL has been developing new approaches for distributed control that can be considered "transactive." The second is to make advances in the development of an advanced modern grid planning and analytics platform to evaluate future grid alternatives as articulated in the DOE's 2015 Grid Modernization Initiative Multi-Year Project Plan. Such a platform would enable modeling of clean distribution systems that are "possible due to many new grid developments including ... proliferation of smart consumer end-use devices on the customer side of the meter" and "new approaches for distributed control . . . and coordination across local intelligent assets."

Since 2014, NREL has been developing a simulation platform, the Integrated Energy Systems Model (IESM), and has used it to demonstrate system impacts of high penetrations of smart consumer devices (including home energy management systems and controllable water heaters) on distribution feeders under various tariff structures. The IESM was used to perform the simulations for the TE Challenge.

The simulations for the TE Challenge were performed on NREL's high-performance computer (HPC), Peregrine, a 2.24-petaFLOPS system. It provides a multifaceted basis for simulating future integrated energy innovations. Peregrine is housed in the $10,000 \mathrm{ft}^{2}\left(930 \mathrm{~m}^{2}\right)$ Energy Systems Integration Facility (ESIF) data center; it is among the world's most energy-efficient computers, with a power usage effectiveness of 1.035. The HPC capabilities are complemented by extensive state-of-the-art visualization capabilities. The ESIF's Energy Visualization Roomwith a high-performance workstation and a high-resolution large-scale display wall-was used for visualizing results from the simulations.

The NREL team was led by Annabelle Pratt, a principal engineer with NREL's Power Systems Engineering Center (PSEC). Fei Ding (PSEC) was the lead for simulations, and Sivasathya Balamurugan from NREL's Buildings and Thermal Systems Center provided support. Emiliano Dall'Anese (PSEC) was the lead architect for the TE control (TEC) approach, and Xinyang Zhou was responsible for the specific design of the control algorithms for these simulations. Dheepak Krishnamurthy from NREL's Strategic Energy Analysis Center (SEAC) led the development and configuration of the co-simulation platform with support from Monte Lunacek from NREL's Computational Sciences Center. Mark Ruth (SEAC) was a key contributor to the analysis of the results, supported by Rune Junker, a collaborator from the Technical University of Denmark (DTU), and Prateek Munankarmi, an intern from South Dakota State University. 


\subsection{Co-Simulation Platform: Design and Interfaces Between Tools}

The performance of the proposed TE approach was evaluated through software simulation using the IESM, as shown in Figure NREL-1. The IESM is a co-simulation framework that integrates simulation of a distribution feeder, buildings (including thermal performance and building appliances), distributed energy resources (DERs) including PV and battery systems, and controllers such as a home energy management system (HEMS) under different markets or tariff structures [1][2]. Although many tools exist for simulating such systems, co-simulation allows a researcher to select independent models for each component in a simulated system. This enables tailoring the set of models to address the fidelity requirements and use case figures of merit that are unique to that problem, while drawing from best-in-class component models. The cosimulation coordinator initiates, manages timing, and supports data exchange among the different components, which can have varying time steps.

The IESM is designed to run in a HPC system to allow the parallel execution of hundreds of instances of complex controllers-for example, HEMS algorithms based on a model predictive control (MPC) approach. The IESM can also be interfaced with residential appliances and rooftop PV systems to perform hardware-in-the-loop simulations [3][4]. The IESM has been used to evaluate the impacts of emerging consumer technologies under different rate structures [5]. Consumer technologies can include home energy management systems, electric vehicles (EVs), battery energy storage systems (BESS), and smart thermostats coupled with air conditioners $(\mathrm{A} / \mathrm{C})$ and water heaters. For the TE Challenge, the controls that are described below in the TE approach section were embedded within the IESM.

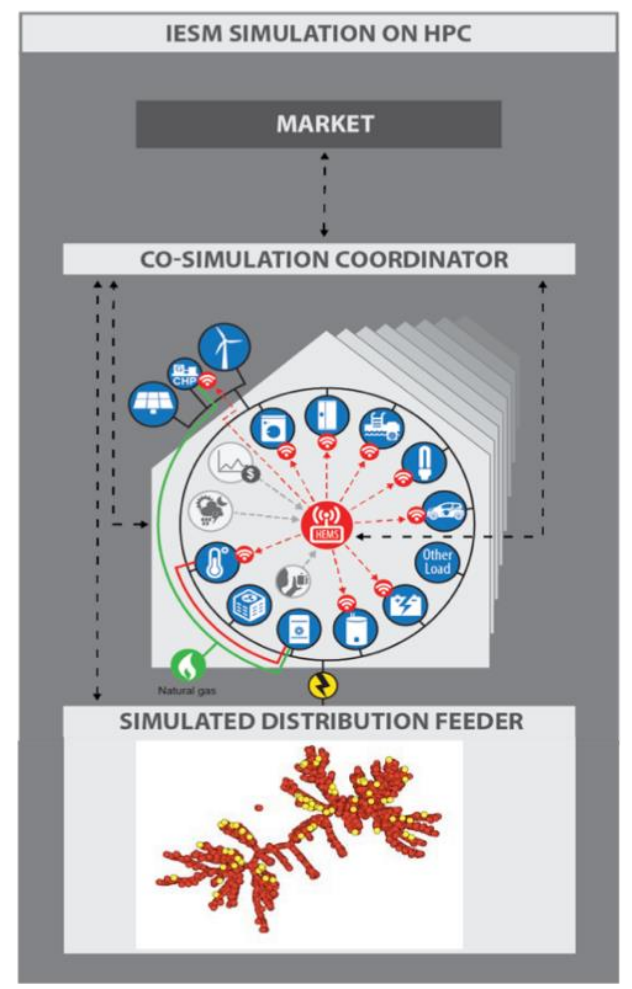

Figure NREL-1. Diagram of the IESM including a co-simulation coordinator, simulated distribution feeder, simulated homes with home energy management systems, and markets. 
Components from the TE Challenge's abstract component model can be mapped closely to the components or agents in the IESM, as shown in Figure NREL-2, which is based on Figure NIST2. The experiment manager in the abstract component model is the co-simulation coordinator in the IESM and it is responsible for initializing the environment and all the components, including the weather component, the supervisory controller component, and the transactive agents. The supervisory controller and the transactive agents are implemented in the IESM environment using Python. When the co-simulation coordinator sets up the IESM environment, communication between the various components is enabled using the IESM data bus. This bus uses ZeroMQ (ZMQ) sockets along with in-memory transfer of information to establish communication between the different components. GridLAB-D represents the grid component along with the resource and local controller components. Communications between IESM and GridLAB-D are established using ZMQ sockets. Analytics is performed as a post-processing step with the data that are collected from the simulation.

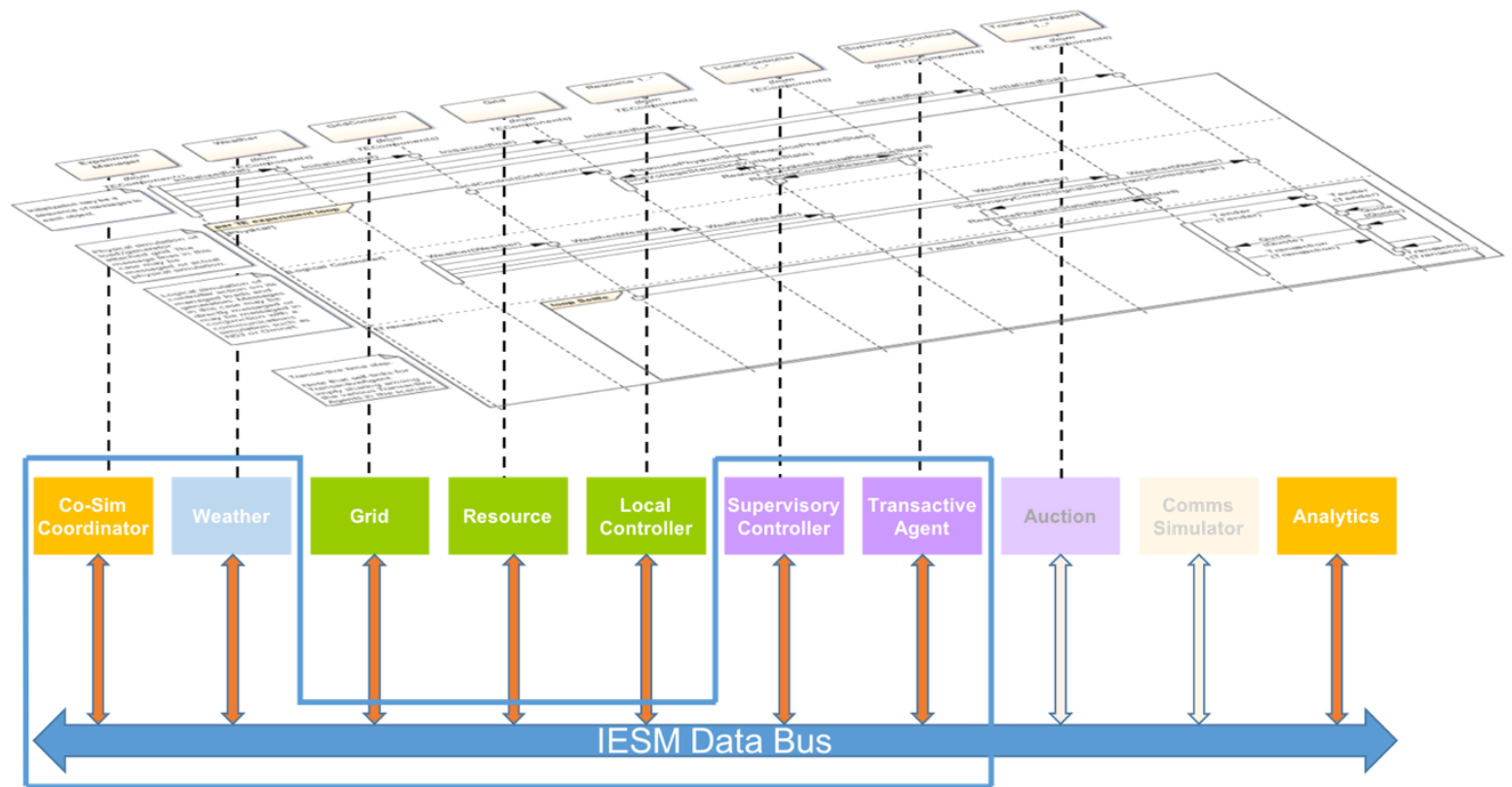

Figure NREL-2 Mapping of IESM components to the abstract component model developed by NIST.

\subsection{Research Approach}

We executed simulations at a 10-second time step using the IESM co-simulation platform and the IEEE 8500 feeder provided by PNNL. We generally followed the four TE Challenge scenarios (baseline sunny day, storm front added, price variation added, TE model for device management), with some exceptions as noted below.

1. Sunny: The baseline sunny day, with no storm front passing through the area. The electricity price is a time-of-use (TOU) rate and there is no transactive energy control. This is a deviation from the National Institute of Standards and Technology (NIST) scenario description; NIST's baseline scenario calls for a constant price. The present research used a TOU rate because that provides a way to compare to scenario 3 , which simulated price-responsive resources. 
2. Cloudy: Adding a storm front to the baseline sunny day, but keeping all else the same as the sunny day. The storm front moves in at 2:30 p.m., and PV power production drops from full sun to $10 \%$ sun in a period of 10 minutes. The storm front moves away and solar insolation increases to full sun from 4:00 p.m. to 4:30 p.m.

3. Price-Responsive Demand (PRD): Adding price-responsive controls to the air conditioner within each home and keeping all other elements the same as for the cloudy day. This results in price-responsive demand, but there are no TE controls; that is, resources do not influence the price.

4. TE Controls (TEC): Adding TE controls, as described below, and keeping all else the same as for the PRD scenario.

We then calculated the common metrics agreed to by the TE Challenge participants. These are presented in the results section below.

\subsection{Transactive Energy Approach}

For the TE Challenge, NREL implemented network-aware TE controls that are able to manage distribution feeder voltages based on real-time optimal power flow in the IESM co-simulation framework [6]. This is one part of a multi-timescale TE controls approach, shown in Figure NREL-3, which we envision will be able to both reduce costs for balancing power supply and demand and provide grid services, such as managing distribution feeder voltages.

The distribution system operator (DSO) operates a multi-timescale transactive market, which could be implemented in a distribution management system (DMS), a distributed energy resource management system (DERMS), or a dedicated platform of an independent market player or coordinator, and it could further use blockchain technology to provide security, transparency, and reduced transaction costs. A DMS is a suite of applications designed to monitor and control the entire distribution network efficiently and reliably. A DERMS extends communications and control to distributed energy resource (DER) systems. The transactive control approach will also need to consider the interaction with the DMS that manages legacy devices, such as capacitor banks and voltage regulators.

Homes participate in the transactive market with the use of home energy management systems (HEMS) that manage appliances and DERs to meet occupants' comfort requirements - including cooling, heating, hot water, and vehicle charging - and minimize their energy cost based on the market price received from the DSO.

Transactive energy has been defined as "a system of economic and control mechanisms that allows the dynamic balance of supply and demand across the entire electrical infrastructure using value as a key operational parameter" [7]. Therefore, most transactive control approaches incentivize load shifts and the use of DERs to reduce the costs of dynamically balancing supply and demand and to limit active (real) power peaks, but few have the capability to regulate voltage - a key responsibility of DSOs. The acceptance, deployment, and use of transactive control approaches to manage power system operation, however, will require demonstrated ability to meet all operational requirements and provide benefits to both DSOs and customers. 


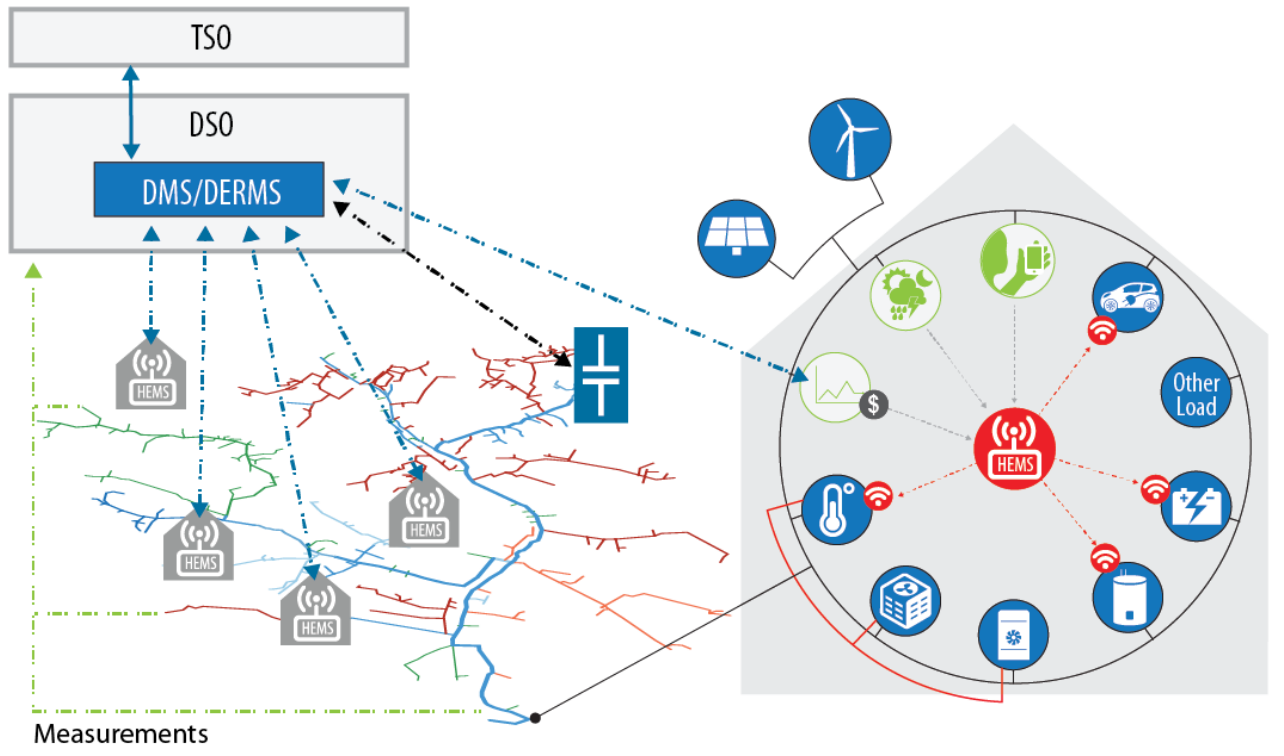

Figure NREL-3 Overview of multi-timescale TE controls approach

We envision a composite pricing structure, illustrated in Figure NREL-4, with (1) an energy price that is determined at a 5-minute to 15-minute timescale based on wholesale prices determined by the transmission system operator and bids by participating houses and DERs; and (2) incentive signals for fast-timescale grid services that are updated on a 1-second to 10-second timescale. For the TE Challenge, we simulated only the calculation of the incentive signals for voltage regulation services and we used the TOU rate for the energy price. A high-level description of the calculation of the incentive signals is provided here, and the reader is referred to [6] for technical details.

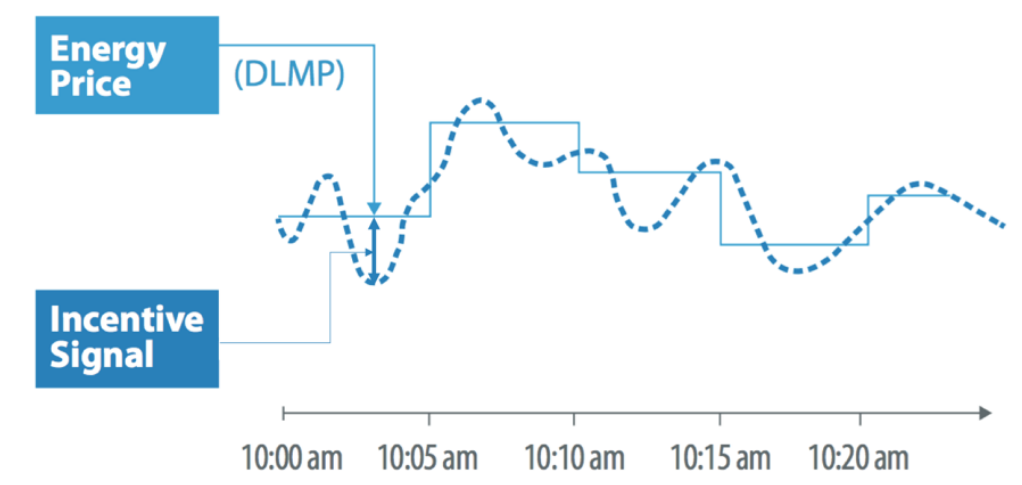

Figure NREL-4 Composite price signal.

There are two levels of controllers: (1) house-level controllers, implemented in HEMS, that manage appliances and DERs to meet occupants' comfort and energy cost objectives based on the TOU rate and the incentive price signal received from the network-level control; and (2) the network-level controller, implemented by the DSO, that determines house-specific incentive price signals to incentivize houses to participate in providing voltage regulation services. The 
network-level control corresponds to the supervisory controller shown in Figure NREL-2, and the house-level controls correspond to the transactive agents. Buildings can support voltage regulation by increasing or decreasing their active power consumption or generation and/or their reactive power sourcing or sinking. Building effect on voltage depends on location within the network, and therefore the incentive price signals - for both active and reactive powers - are determined by taking into account the physics of the distribution network (i.e., it fully accounts for $\mathrm{AC}$ power flows in three-phase unbalanced distribution networks using a linearized threephase power flow model [8]), measured feeder voltages, and predicted power usage information for the houses. These predictions could be either supplied by the house-level controls or estimated by the network-level controls. For the TE Challenge, we implemented the former because, in the field, each house-level controller would have access to more data and could produce a more accurate estimate of future power use. This approach also reduces the complexity of the network-level controller which would simplify implementation by a DSO but would impose increased communications requirements. For the TE Challenge, PV inverters and air conditioners were controlled at the house level.

The controls at the network (DSO) level are responsible for the proper design of the incentive price signals to be issued to the house-level controls so that the network-level objectives are met, as described in [6]. The objective used for the TE Challenge includes both customer-oriented and network-oriented objectives and constraints. It aims to minimize the total customer discomfort and electric energy cost while preventing voltage violations from occurring across the feeder.

This is a closed-loop approach in which the network-level control determines the incentive price signals for voltage regulation - for both active and reactive powers - that are issued to the houses every 10 seconds. The network-level controller issues both a current (real-time) incentive price signal and the predicted incentive price signals for the upcoming hour (at 15-minute granularity) based on predicted load for the coming hour (at 15-minute granularity). The house-level controls respond to the combination of these incentive prices and the TOU price by adjusting power consumption or generation; the resulting net power consumption of the houses determines the voltages across the distribution network. These voltages, in turn, are inputs to the network-level control [6]. Although the controls are executed frequently, the control algorithms are simple and can be executed in a short time, because they are based on an online optimization approach in which the optimal solution is reached within several time steps, as described in [6].

The house-level controllers (HEMS) are implemented with controls at the device level; that is, the PV inverter and air conditioner each has a dedicated controller. The air conditioner controllers determine the optimal temperature set points for these thermostatically controlled loads for the coming hour (at 15-minute granularity) based on the internal models of these devices, the TOU price and incentive price signals, and the forecasted outdoor temperature information. The comfort band is set to $\pm 5^{\circ} \mathrm{F}$, i.e., the air conditioner controllers are constrained to keep the set points within $\pm 5^{\circ} \mathrm{F}$ of the desired temperatures. The internal air conditioner models are estimated as linear-regression models, based on data from simulations performed without active house-level controls [3]. The objective of these controllers is to minimize a weighted sum of the cost of electric energy and the occupants' discomfort. The discomfort is expressed as the deviation of the predicted air temperature from the desired temperatures set by the customers. The thermostatic controls implemented in GridLAB-D regulate the air 
temperature to the optimal set points, and the power consumption of the air conditioners therefore also depends on the GridLAB-D thermostatic controls. Air conditioner optimizations are executed every 15 minutes but in an asynchronous way-i.e., at every 10-second time step a subset of the air conditioners performed their optimizations - to avoid the negative effect caused by significant sudden power changes.

The PV inverter controllers compute the active and reactive PV power outputs for the coming hour (at 15-minute granularity) based on an optimal trade-off between the income earned by providing reactive power provisioning and the loss of income due to active power curtailment, while respecting the apparent power rating of the inverter. The PV inverter optimizations are executed every 10 seconds because inverters can respond very quickly. Note that only the PV inverter controllers use the incentive signals for reactive power, and the PV inverters at a node are incentivized to consume more reactive power when an overvoltage problem occurs, and to generate more reactive power when an undervoltage problem occurs, across the whole distribution network.

At each 10-second time step, the house-level controllers (HEMS) provide the network-level controller (DSO) with an estimate of the power that each house will consume during the next hour (at 15-minute granularity) based on the decisions made by the PV-inverter and air conditioner controllers.

For the PRD scenario, the same air conditioner controllers are used as for the TEC scenario, but they respond only to the TOU price. No network-level controller or PV inverter controllers are used for the PRD scenario.

\subsection{Research Results}

Figure NREL-5 shows the solar insolation and outdoor temperature data that were provided by Pacific Northwest National Laboratory (PNNL) for use by TE Challenge teams. Only the Sunny scenario uses the sunny weather data and the Cloudy, PRD, and TEC scenarios use the cloudy weather data. Figure NREL-6 shows the TOU rate that is used, as defined for the TE Challenge and incorporated in GridLAB-D by PNNL.
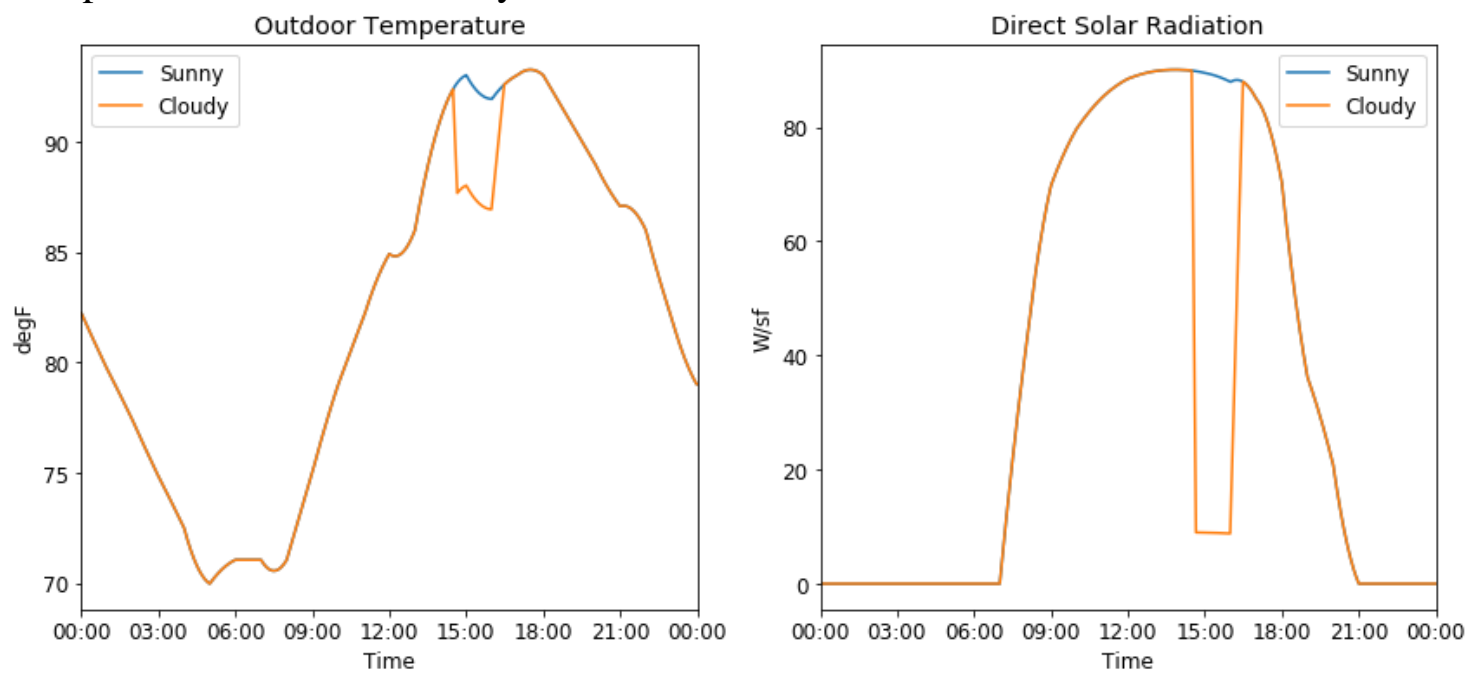

Figure NREL-5 Weather data used for simulations. 


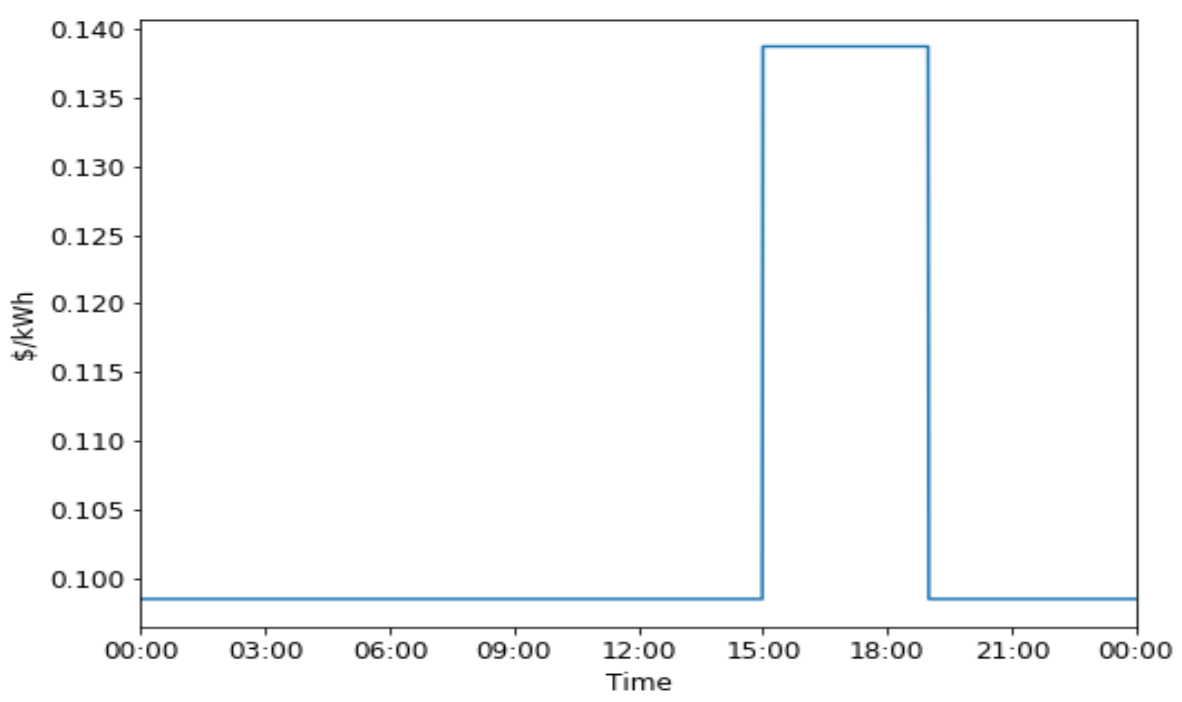

Figure NREL-6 The TOU rate used for simulations.

The TOU rate was used for the energy price and therefore the network-level controller calculates only incentive price signals for voltage regulation services. The objective of the network-level control in the TE controls approach is to minimize the total customers' electric energy cost and discomfort (as described in the house-level control) while preventing voltage violations from occurring across the feeder. According to ANSI C84.1-2016, an overvoltage violation occurs when a voltage exceeds 1.05 p.u., and an undervoltage violation occurs when a voltage is less than 0.95 p.u. The results presented below show the effectiveness of the proposed TE controls approach. Although the IESM was configured to simulate the entire day for each scenario, results are presented here for the time period from 6 a.m. to 6 p.m., which captures the period of peak PV production and the cloud cover event. For the PRD scenario, the power flow could not be solved after the TOU price dropped at 7 p.m., and therefore the PRD simulation stopped at that time. This is because the house-level controllers reduce the load during the peak TOU price period; therefore, at the end of the peak TOU price period, there is a significant increase in load, often referred to as the rebound effect. This illustrates potential challenges with uncoordinated price-based control, as discussed in [5]. When house-level controls all respond to the same price signal, the load responds in a synchronized way, and this can result in severe load steps that can cause issues on a real distribution feeder as well. A similar load rebound is observed for the TEC scenario, but in this case, the network-level controller mitigates the impact and the power flow is solved.

Figure NREL-7 shows the average voltage magnitude at all houses, and Figure NREL-8 shows the values of all house voltages for all scenarios where the colors indicate the distribution of voltages, from the mean (in red) to the range (in gray) within which $95 \%$ of all voltages fall. The horizontal dotted lines in Figure NREL-8 at 1.04 p.u. and 0.96 p.u. indicate the voltage range within which the network-level controller aims to keep the voltages, to allow some margin from the ANSI standards. For the Sunny, Cloudy, and PRD scenarios, the average voltage increases during the morning hours, peaking at approximately 9:00 a.m., because the PV generation increases when the load is still low. Load is low because outdoor temperatures are too low to require operating air conditioners. Similarly, for the Sunny, Cloudy, and PRD scenarios, the average voltage decreases during the late afternoon hours, because the PV generation decreases 
when the load is still high. The average voltage under TEC is more consistent during the course of the day. It becomes lower between 7 a.m. and 2 p.m. when voltage is generally high for the other scenarios and it becomes higher after 4:30 p.m., when voltage is generally low for the other scenarios. PV generation significantly reduces due to the cloud cover from 2:30 - 4:30 p.m., and average feeder voltage decreases for the cloudy and PRD scenarios, but it first decreases and then increases for the TEC scenario. The drop in voltage at 2:30 p.m., at the start of the cloud cover event, is smaller for the TEC scenario than for the Cloudy and PRD scenarios.

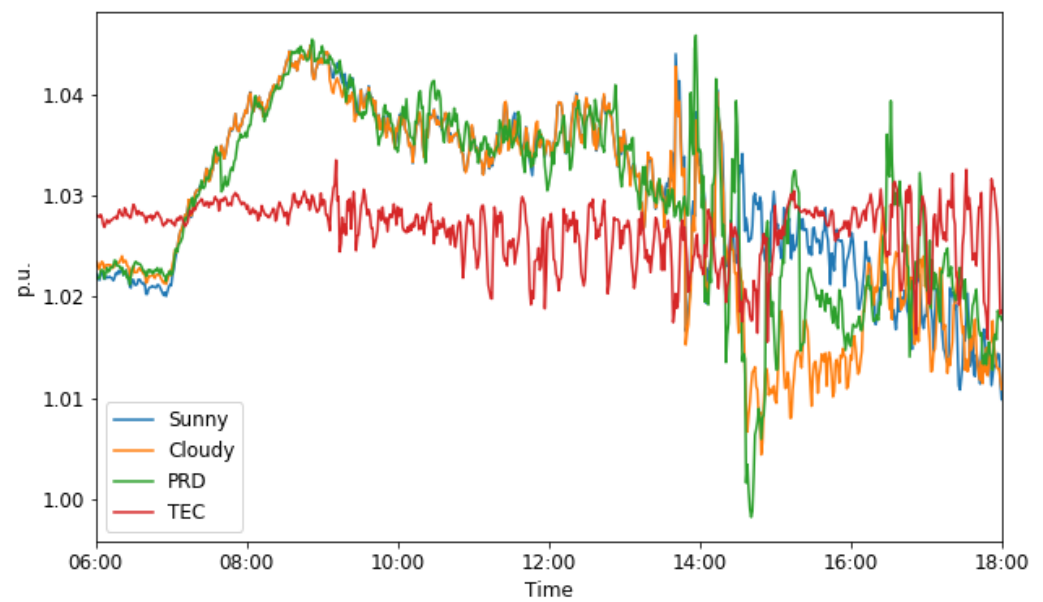

Figure NREL-7 Average voltages for all houses.
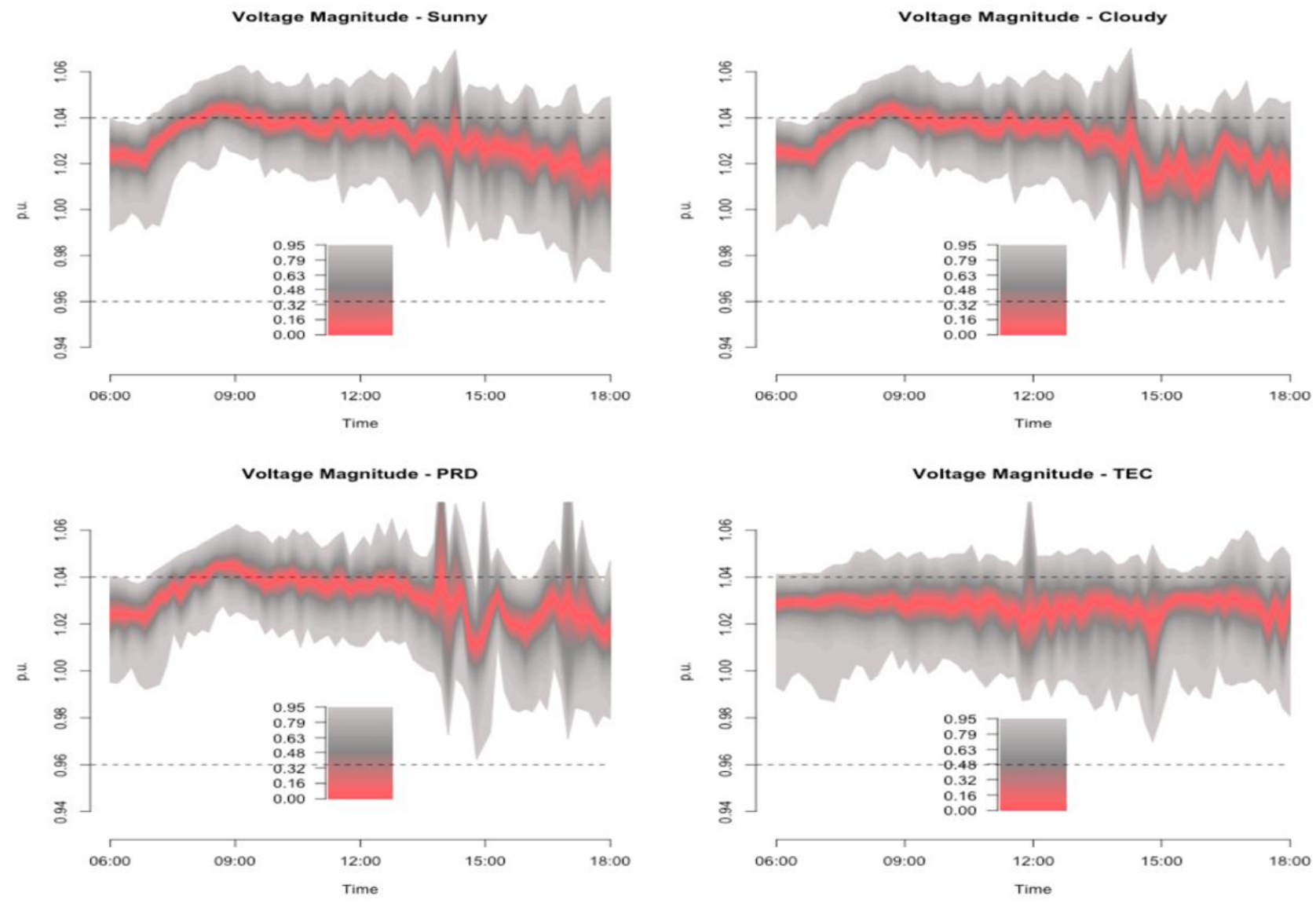

Figure NREL-8 Voltages at all houses for all four scenarios 
Figure NREL-9 shows the proportion of houses with overvoltage violations, and Figure NREL10 shows the proportion of houses with undervoltage violations. Figure NREL-11 shows the cumulative number of voltage violations, which is counted as the accumulated number of both overvoltage and undervoltage violations. For the Sunny, Cloudy, and PRD scenarios, a significant proportion of houses - up to $60 \%$ - experience overvoltage violations during the morning hours. The voltage spikes again between 1:00 p.m. and 2:00 p.m., just before the cloud cover occurs and when PV generation is at its peak, and additional spikes occur at 4:00 p.m. as the cloud cover disappears. The TEC scenario achieves a significant reduction in overvoltage violations between 9:00 a.m. and 1:00 p.m. because houses are responding to incentive signals provided by the network-level controller to reduce voltage violations.

Voltages drop during the storm front for the Cloudy, PRD and TEC scenarios due to reduced PV generation, and this results in fewer overvoltage violations than for the Sunny scenario during this time.

In summary, the TEC scenario reduced accumulated voltage violations by approximately $80 \%$, compared with the cloudy scenario.

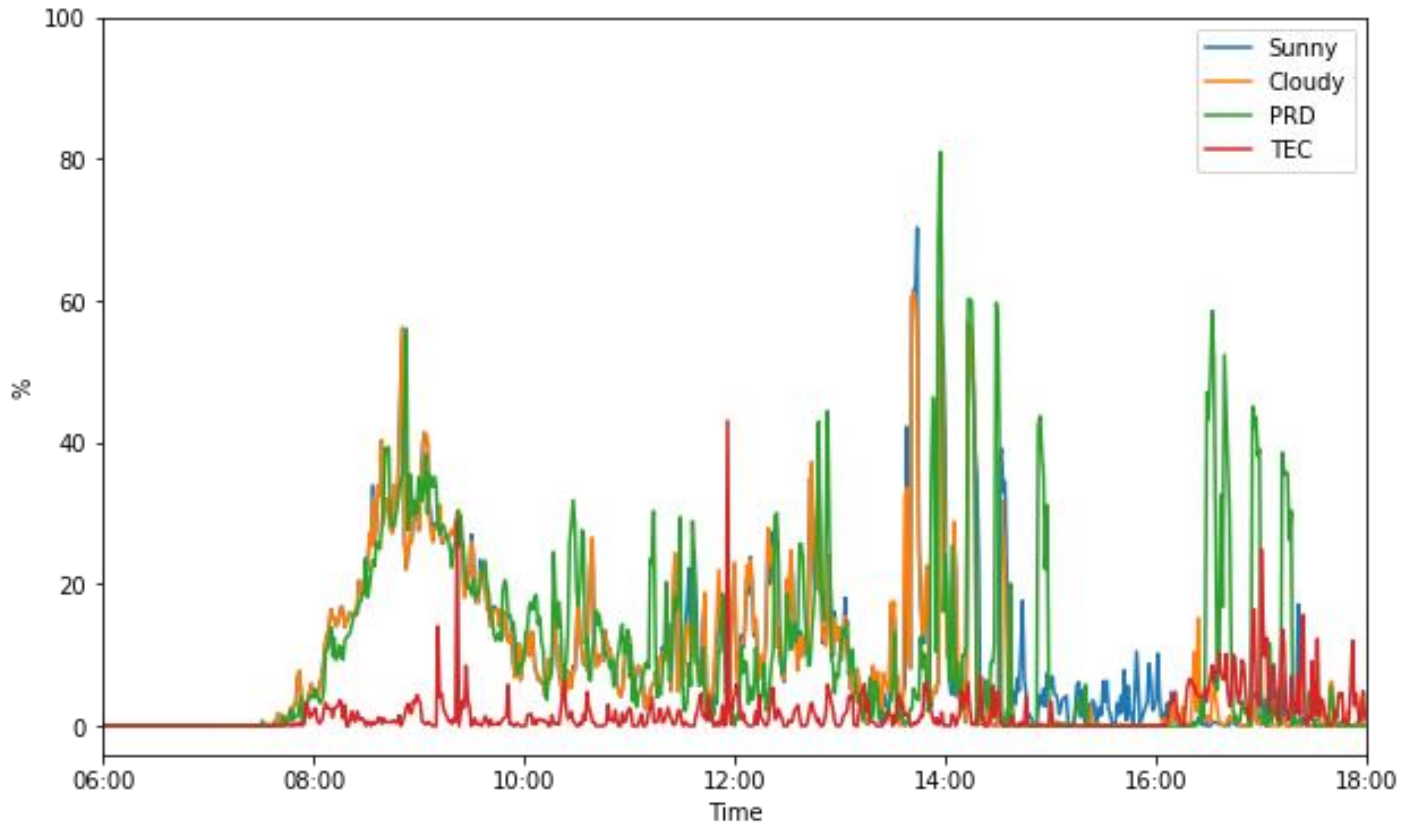

Figure NREL-9 Proportion of houses with overvoltage violations. 


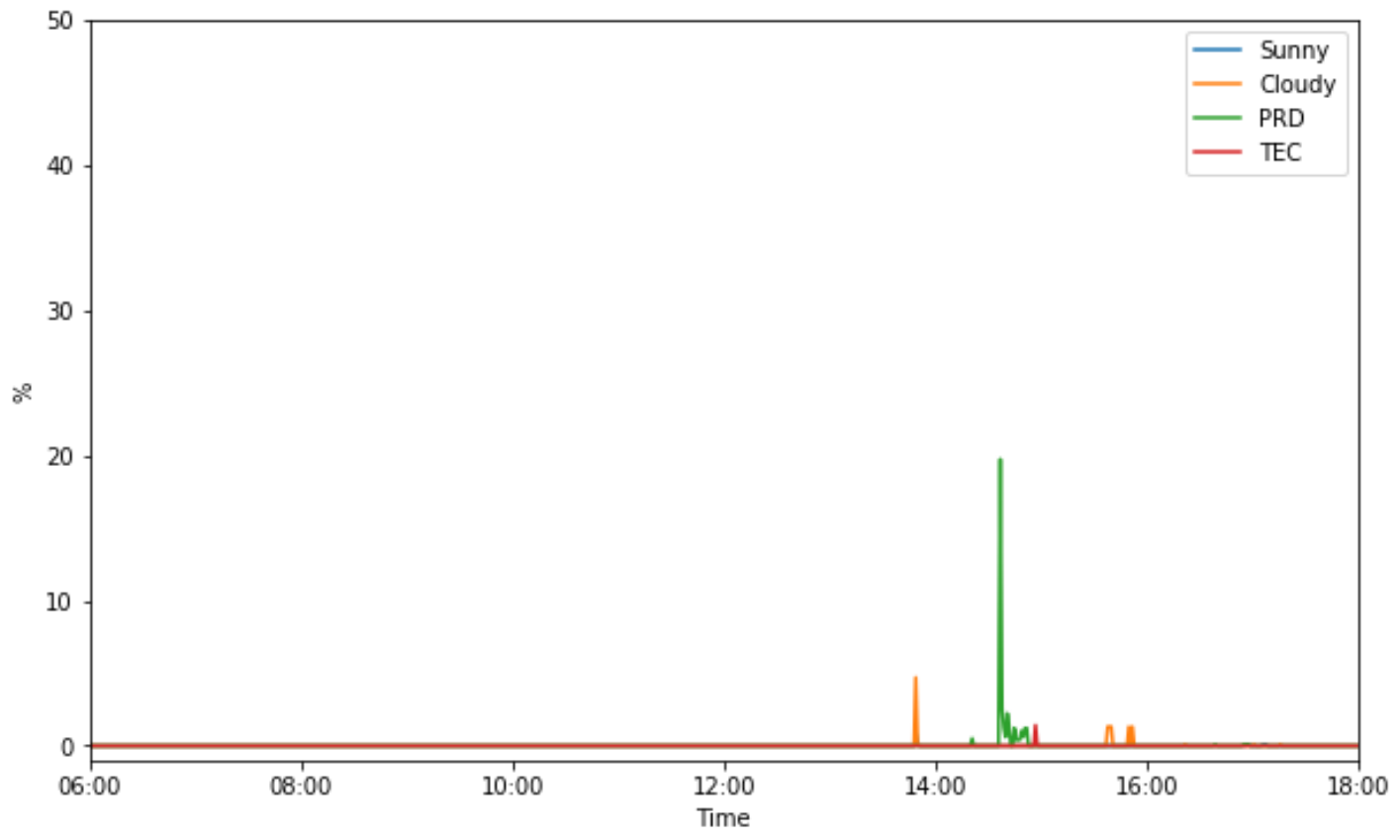

Figure NREL-10 Proportion of houses with undervoltage violations.

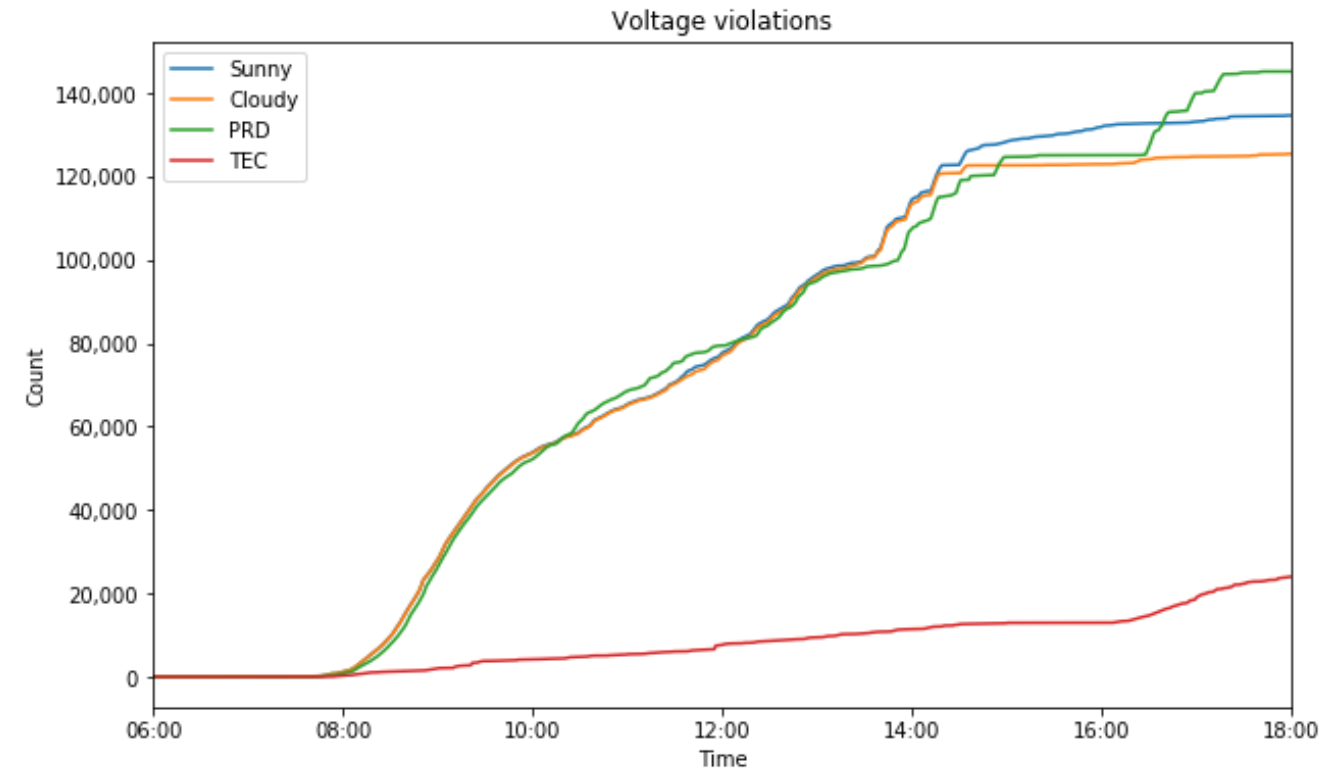

Figure NREL-11 Cumulative number of voltage violations.

Our TEC approach reduces voltage violations by calculating and communicating incentive price signals for both active and reactive power to all the houses. Figure NREL-12 and Figure NREL13 show the incentive prices calculated by the network-level control for active and reactive power, respectively. The colors indicate the distribution of prices, from the mean (in red) to the range (in gray) within which $95 \%$ of all prices fall. The distribution in prices at any given time illustrates the way in which a specific home's power generation/consumption impacts the voltage based on its location. Therefore, some houses might be more strongly incentivized to provide reactive power, for example, than others, because the provision of reactive power at those specific locations would yield greater voltage improvements than at other locations. From about 
8:00 a.m. until about 2 p.m., the incentive signals become negative, meaning that households are motivated to help reduce voltage by using more power for air conditioning, curtailing PV active power generation and by having the PV inverters absorb reactive power. At about 2:30 p.m., when the cloud cover moves in, the incentive signals increase sharply towards positive values, meaning that households are incentivized to consume less power for air conditioning, and for PV inverters to source reactive power, to help boost voltage. The incentive signals remain positive during the peak TOU price period, which starts at 3 p.m., to reduce household energy costs.

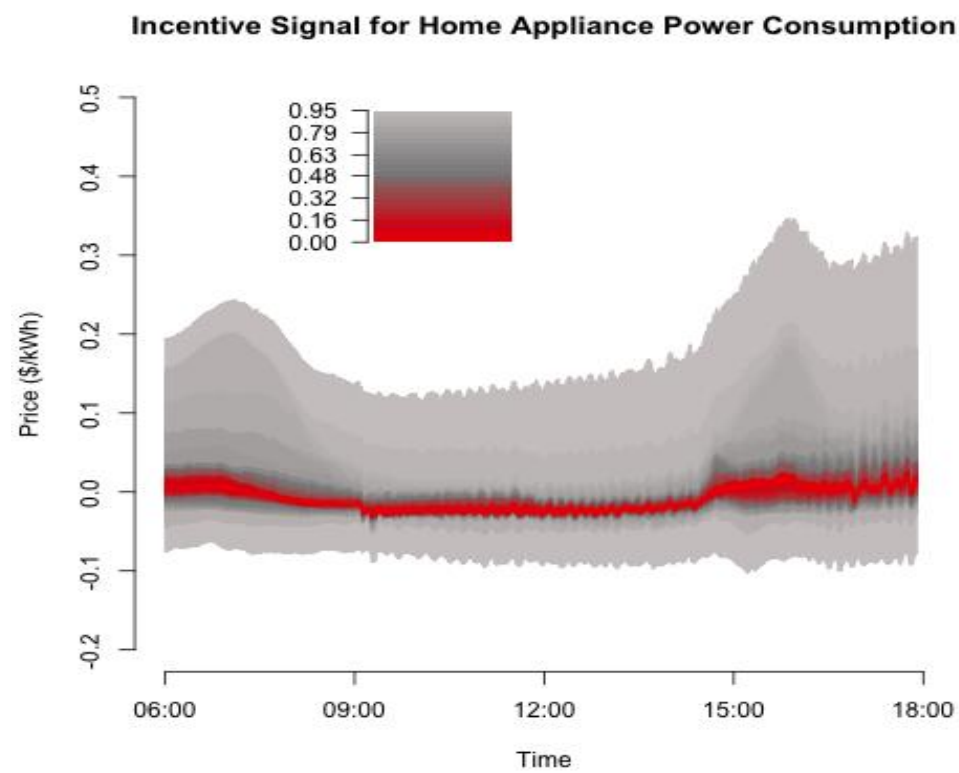

Figure NREL-12 Incentive prices for active power generated by the network-level control.

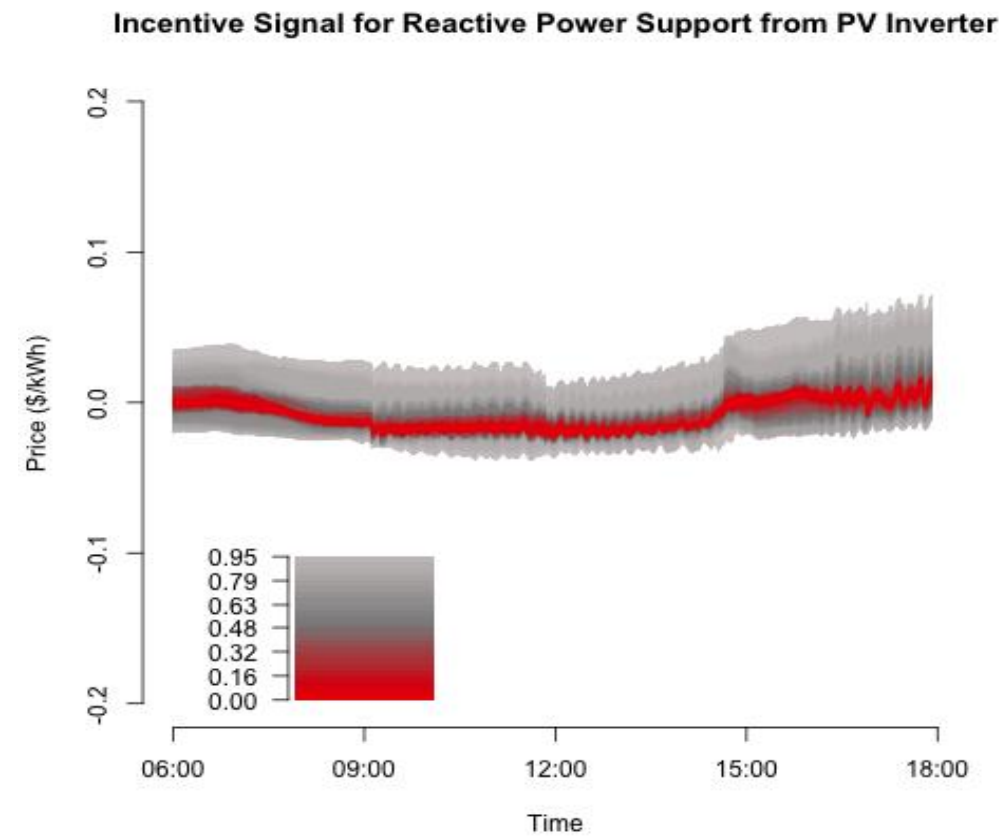

Figure NREL-13 Incentive prices for reactive power generated by the network-level control. 
Figure NREL-14 shows the average net power - which includes all loads and PV systemsconsumed by the houses for the four different scenarios. The net load power is higher for the TEC scenario between 9 a.m. and 2 p.m. because the PV inverters curtail their production in response to the incentive signals. This is shown in Figure NREL-15 which shows the average active and reactive power output of the PV inverters. The PV output power is determined solely by the solar insolation for the first three scenarios. For the TEC scenario, however, the inverters respond to the prices issued by the network-level control [6]. In the TEC scenario, the PV inverters curtail their active power output between 9 a.m. and 2 p.m., and they sink reactive power in response to the incentive signals, which reduces system voltages and counteracts the voltage increase due to high PV production. When cloud cover reduces PV output, the inverters generate reactive power into the feeder to counteract the decrease in voltage.
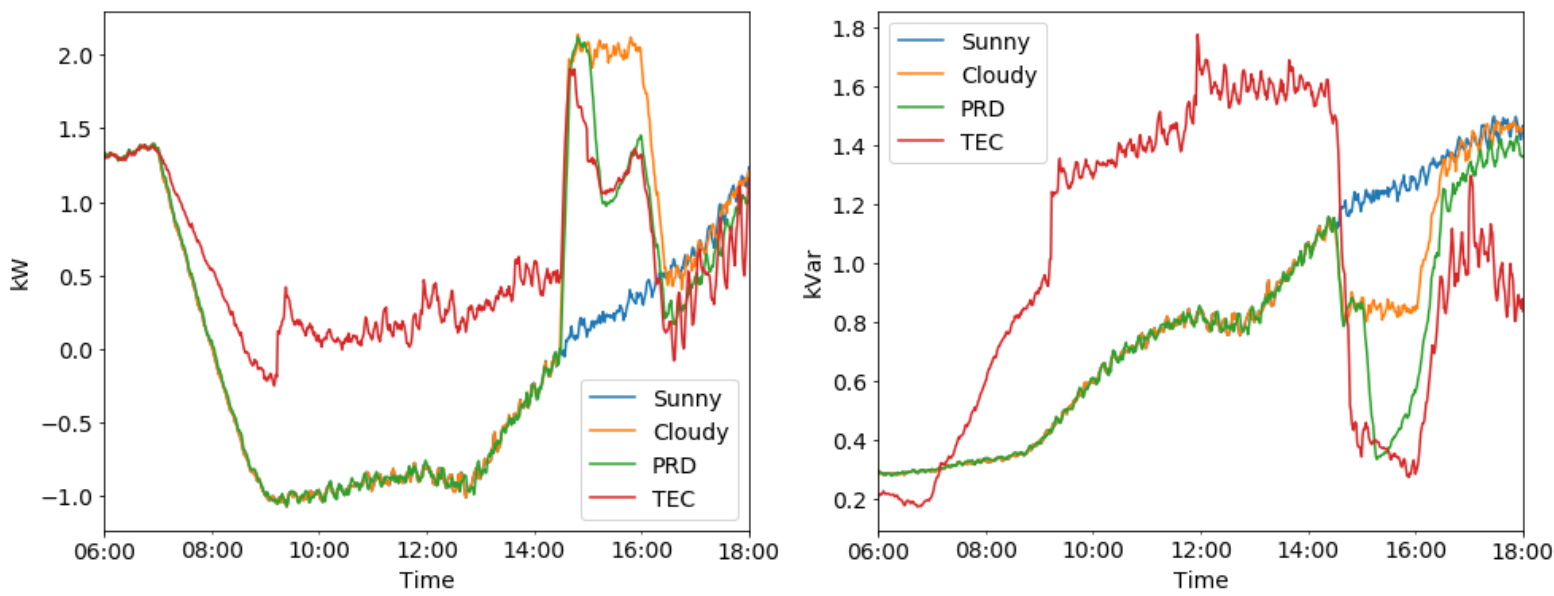

Figure NREL-14 Average active (left) and reactive (right) net power consumed by houses.
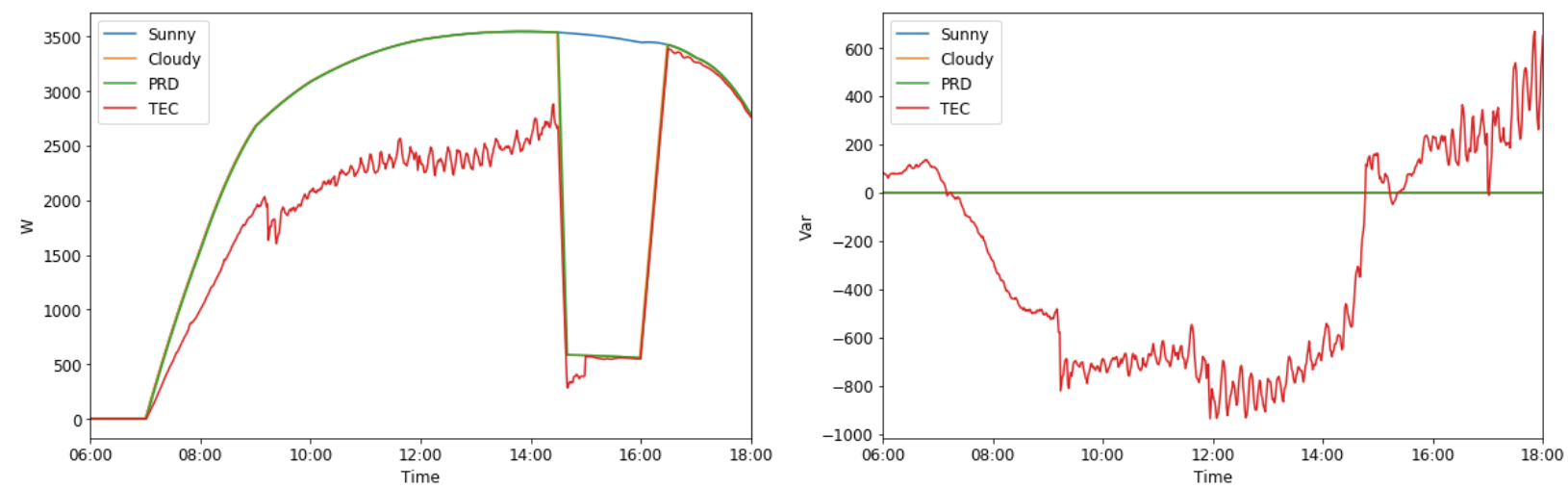

Figure NREL-15 Average active (left) and reactive (right) power output of PV inverters.

The negative active power incentive signal between 9 a.m. and 2 p.m. also incentivizes the air conditioners to consume more power to reduce the voltage. Figure NREL-16 shows the average value of house air temperatures for all houses along with the average desired set point for all houses. Houses have different desired temperature profiles, which are provided by PNNL in the GridLAB-D file. In the morning, the house temperatures are lower than the desired set point. In the afternoon and evening, the house temperatures follow the desired setpoint closely for the 
sunny and cloudy scenarios. For the TEC scenario, the air conditioners respond to the incentive signals between 9 a.m. and 2 p.m. by decreasing the temperature which increases the power consumption. This is shown in Figure NREL-17 where the average power used by the air conditioners are shown for the four different scenarios.

When the storm front arrives at 2:30 p.m., the air conditioner power drops for the Cloudy, PRD and TEC scenarios due to cooler outside temperatures. The average air temperatures are higher for both the PRD and the TEC scenarios than for the two other scenarios between 3 p.m. and 6 p.m. when the TOU rates are at their peak because their air conditioner controls take energy cost into consideration. This increase in air temperature results in a further drop in air conditioner power for the PRD and TEC scenarios.

The changes in air temperature that result from the controls in the PRD and TEC scenarios are less than $2{ }^{\circ} \mathrm{F}$ compared to the Cloudy scenario average temperatures and therefore comfort is maintained for occupants.

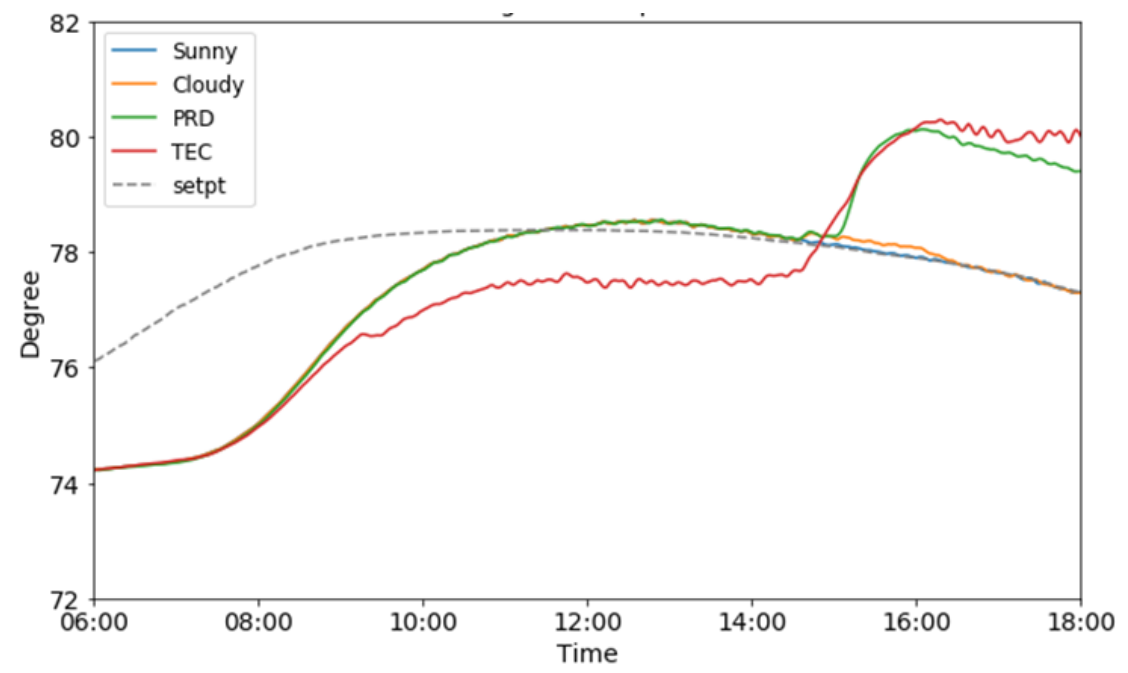

Figure NREL-16 Average house air temperatures and average desired setpoint.

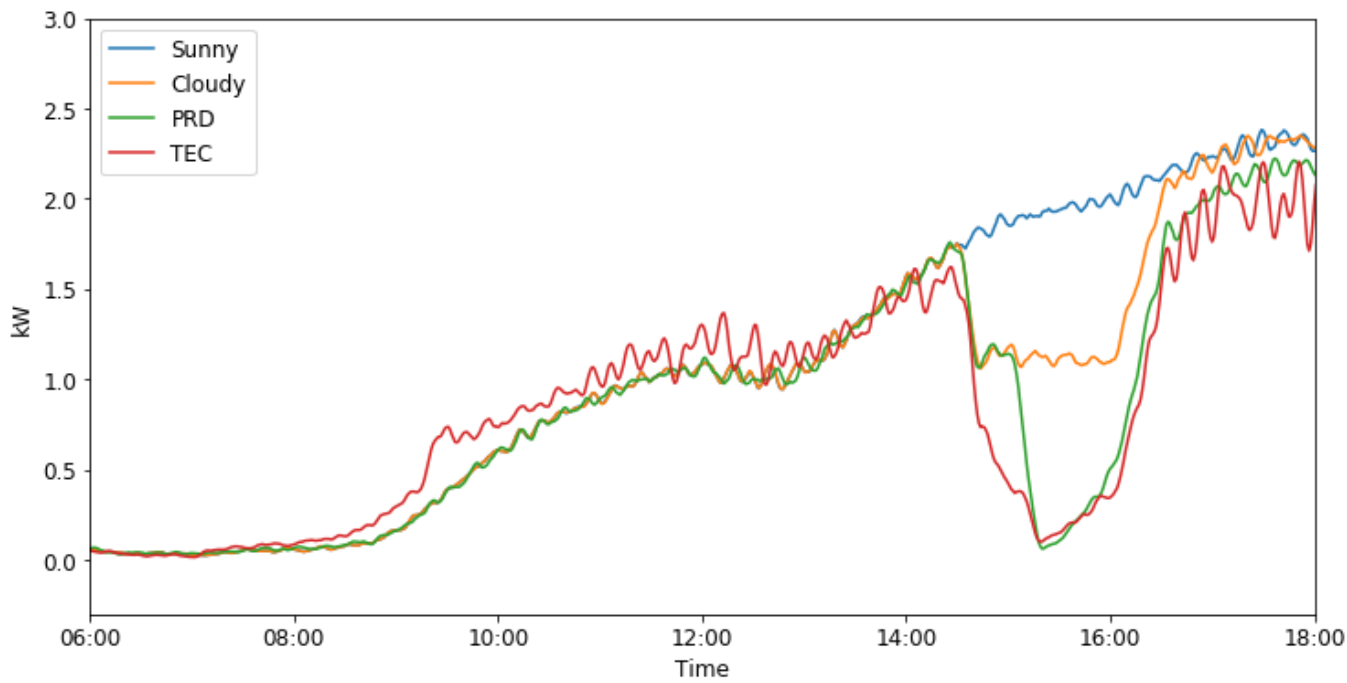

Figure NREL-17 Average power consumed by air conditioners. 
Figure NREL-18 shows the cumulative average energy cost for houses for all scenarios. For the Sunny, Cloudy and PRD scenario, the energy cost is calculated assuming net metering as

$$
\text { Cost }=\left(P_{\text {House }}-P_{P V}\right) \text { TOU }=P_{\text {House }} \text { TOU }-P_{P V} \text { TOU }
$$

where $P_{\text {House }}$ is the power consumed by the house loads, $P_{P V}$ is the power generated by the rooftop PV system and TOU is the TOU price, shown in Figure NREL-7.

The cost calculation for the TEC scenario is more complicated because the houses need to be compensated for the curtailment of PV and the provision of reactive power support. The cost is therefore calculated as

$$
\operatorname{Cost}_{T E C}=P_{\text {House }}(T O U+\alpha)-P_{P V}(T O U+\alpha)-\left(P_{P V}-P_{P V}, \max \right) \alpha-Q_{P V} \beta
$$

where $\alpha$ is the active power incentive price-shown in Figure NREL-12, $\beta$ is the reactive power incentive price-shown in Figure NREL-13, $P_{P V, \max }$ is the active power that would have been generated by the rooftop PV system if it were not curtailed; and $Q$ is the reactive power provided by the rooftop PV system. The four different cost terms for the TEC scenario are shown in Figure NREL-19.

The cumulative average energy cost for houses during the time from 6 a.m. to 6 p.m. are the same for the TEC scenario as for the Cloudy scenario. The cumulative cost drops for the Sunny, Cloudy and PRD scenarios between 9 a.m. and 2:30 p.m. because the PV generation exceeds the load, as shown in Figure NREL-14. The cumulative cost for the TEC scenario also drops, albeit at a lower rate, even though the average net house power is a small positive value during most of this time. This is because of the variation both in house power-i.e., a significant proportion of houses still export power during this time - and in the incentive signals, so that the average of the product of the net house power and the cost of electricity $(T O U+\alpha)$ is negative most of this time. In addition, the cost is reduced by the compensation that is provided for PV curtailment and reactive power support. More extensive analysis is required over a longer time period to determine the value of compensation for curtailment and reactive power support that would ensure fair compensation for all home owners to participate in voltage regulation services. The cumulative cost sharply increases between 2:30 p.m. and 4 p.m. due to the cloud cover which reduces the PV generation for the Cloudy, PRD and TEC scenarios. The Sunny scenario has the lowest cumulative cost because it is able to generate more PV power. The PRD scenario has a lower cost than the Cloudy scenario because of the reduced air conditioner load during the TOU peak price period. 


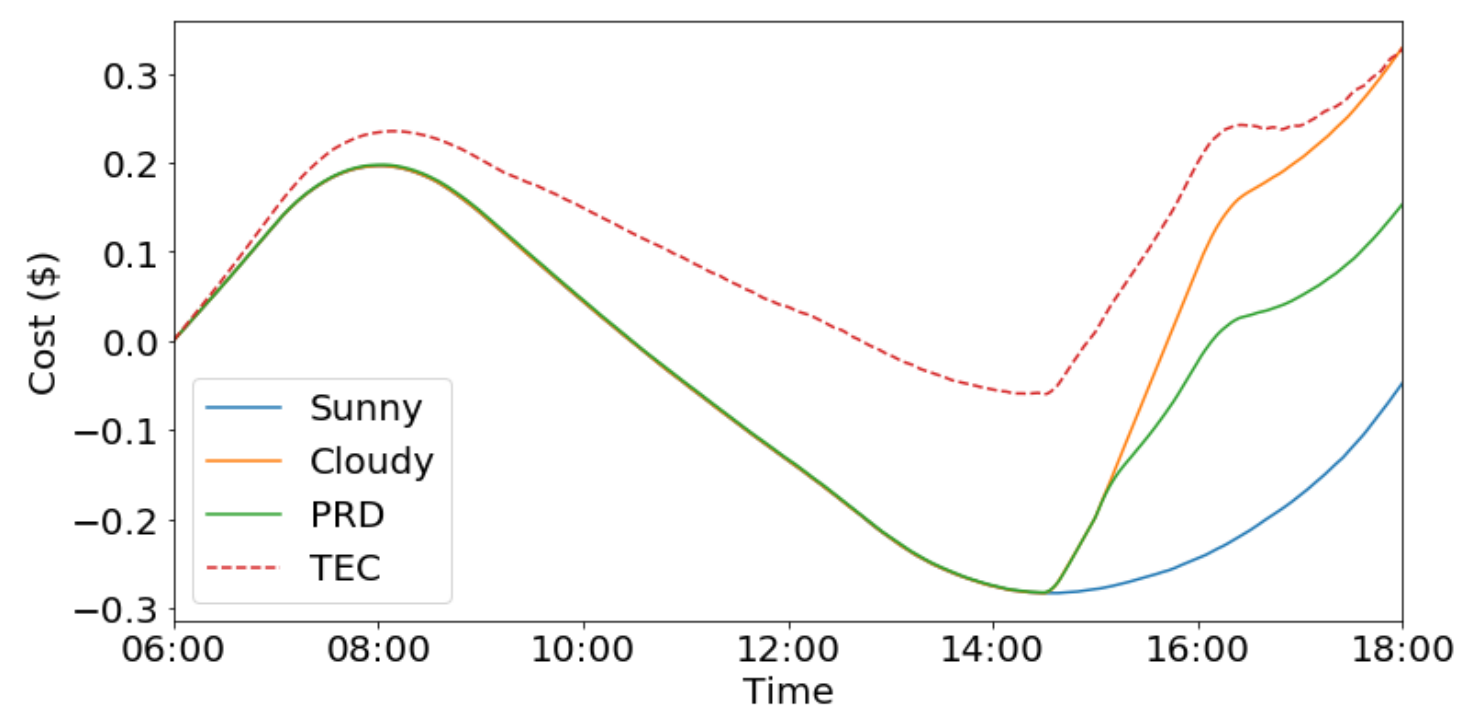

Figure NREL-18 Cumulative average energy cost for houses.

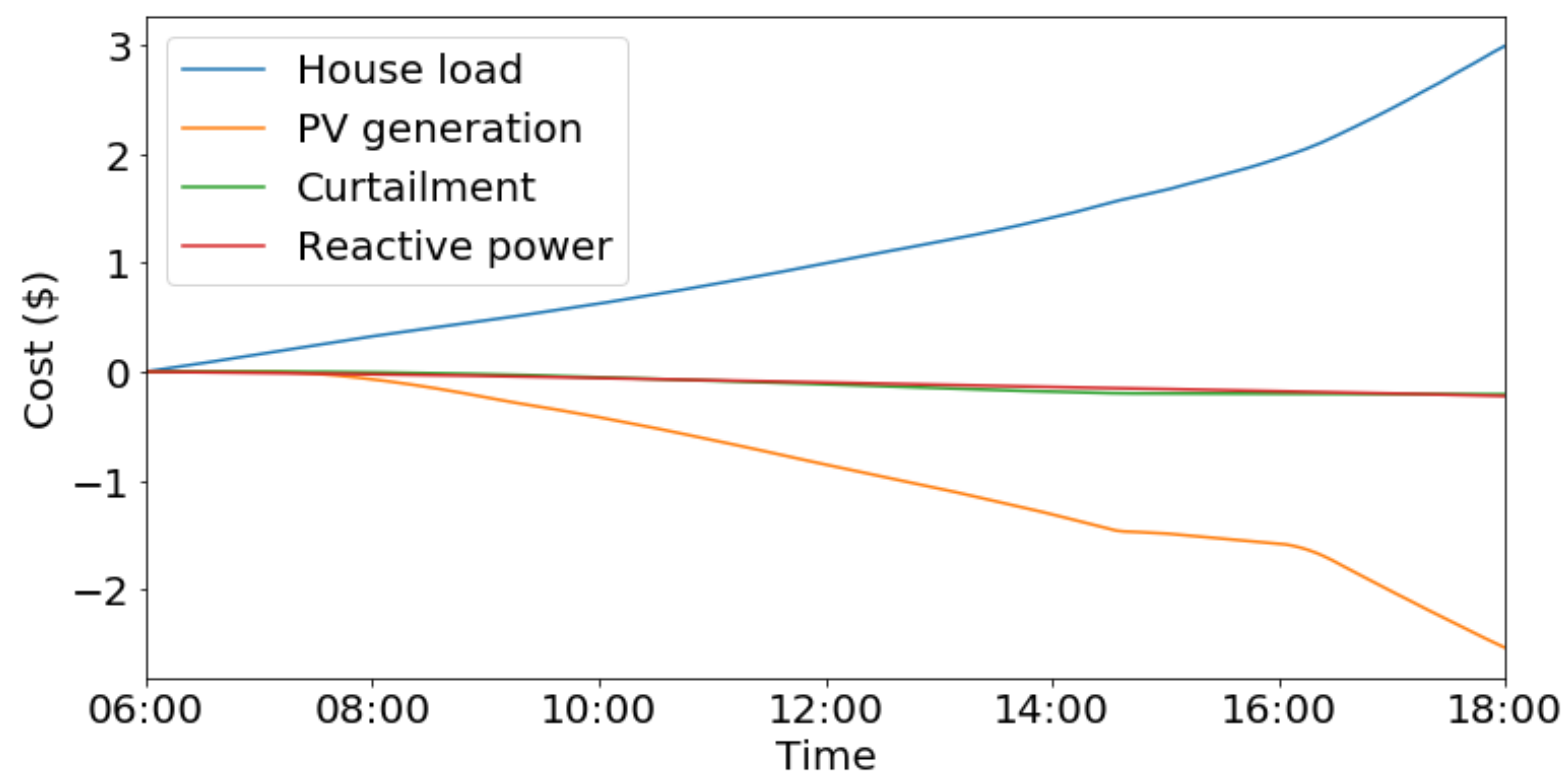

Figure NREL-19 Cumulative average energy cost components for the TEC scenario.

Figure NREL-20 shows a violin plot of costs for all scenarios showing the distribution of costs across the population of houses. It includes an embedded box plot showing the median (central dot), interquartile ranges from the first quartile to the third quartile (thick lines) and $95 \%$ confidence interval (thin lines). The TEC scenario exhibits more outliers in energy cost and addressing this is an area for future work. 


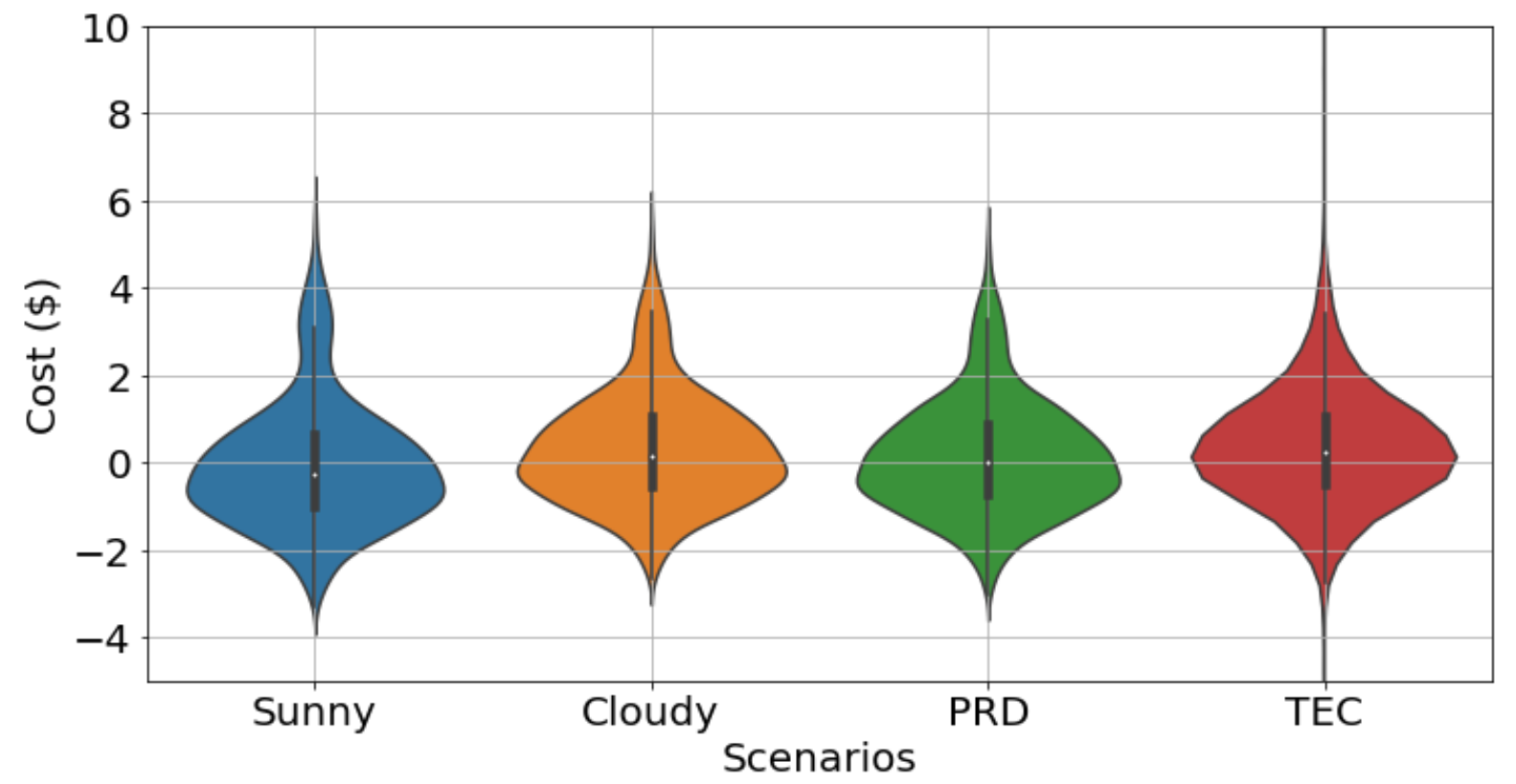

Figure NREL-20 Averages and ranges of energy costs for houses. The TEC scenario has four additional outliers that are not shown at: $\$ 20, \$ 12.76, \$-9.56$, and $\$-27.22$.

Figure NREL-21 shows the total number of capacitor operations and the total number of voltage regulator tap changes, respectively. About $15 \%$ fewer capacitor bank operations and about $20 \%$ fewer voltage regulator operations occur in the TEC scenario than in the Cloudy scenario. For the PRD scenario, there is a significant increase in operations starting at 4:30 p.m., when the cloud cover clears, PV generation increases abruptly, and voltages increase significantly. This is exacerbated by the house-level controllers that decrease load during the TOU peak price period which starts at 3 p.m., as shown in Figure NREL-8 and Figure NREL-9.
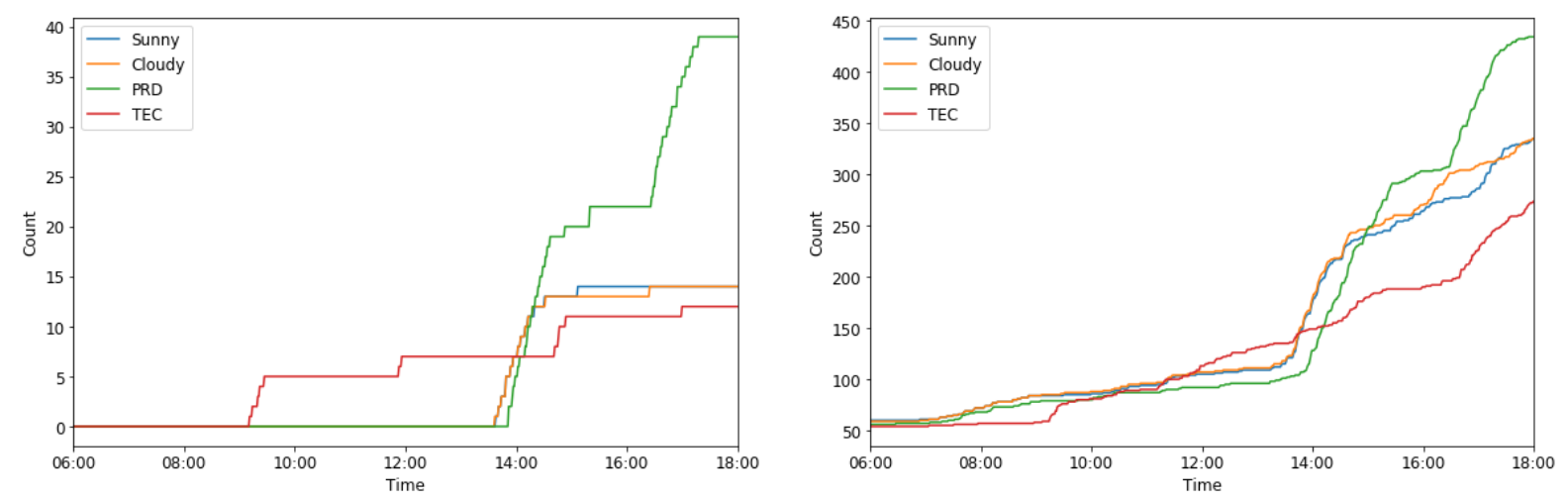

Figure NREL-21 Number of capacitor operations (left) and number of voltage regulator tap changes (right).

Figure NREL-22 shows the active and reactive power at the substation. The sudden changes in PV generation due to the storm front result in significant changes in substation power with very high ramp rates. Between 9 a.m. and 2 p.m., the active power and reactive power draw is higher 
for the TEC scenario than the other scenarios because the PV inverters are curtailing generation and sinking reactive power; conversely, the reactive power draw is lower for the TEC scenario than the other scenarios after 3 p.m. because the PV inverters are sourcing reactive power.
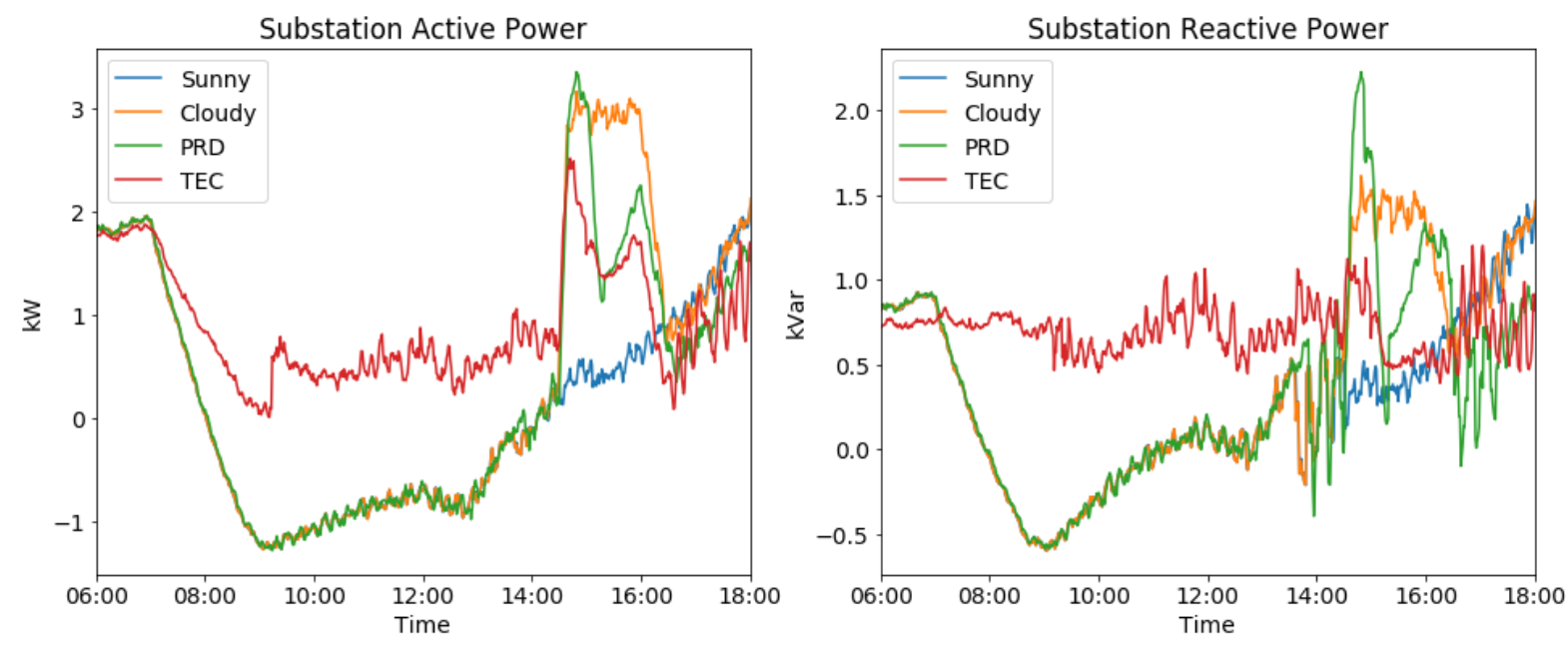

Figure NREL-22 Substation real (left) and reactive (right) power.

\subsection{Discussion}

Existing literature on transactive control mostly focuses on balancing supply and demand without considering voltage regulation, which is a key responsibility of DSOs. In our view, the acceptance, deployment, and use of transactive control approaches to manage power system operations will require demonstrated ability to meet all operational requirements and provide benefits to both DSOs and customers. NREL is therefore developing a TE control approach that uses a composite pricing structure with an energy price that is determined at a 5-minute to 15minute timescale and incentive signals for fast-timescale grid services that are updated on a 1second to 10-second timescale. The TE Challenge teams selected a use case focused on voltage issues and the NREL team focused on simulating only the calculation of the incentive signals for voltage regulation services. We used the TOU rate for the energy price.

Our proposed TEC approach for voltage regulation is network-aware, that is, the network-level control includes a power flow model of the distribution feeder. Our method has an advantage over other network-aware approaches because others either require complex communication or they prescribe the behavior of the control nodes (the house-level controllers, in our case), which might be feasible only for utility-owned devices, not ones owned by customers who are not obliged to behave in a certain way. In addition, our proposed approach uses prices for both active and reactive power, which is likely to better manage voltage than other approaches that consider only active power.

The simulation results show that the proposed TE controls for voltage regulation services are able to limit the number of voltage violations across the distribution power network and maintain the building occupants' comfort. Under the TEC scenario, the number of voltage violations were reduced by about $80 \%$ from the Cloudy scenario and even further from the PRD scenario, whereas differences in air temperature (averaged over all the houses) between the TEC and Cloudy scenarios were less than $2^{\circ} \mathrm{F}$ and thus the occupants' comfort was impacted only 
minimally. The number of capacitor bank and voltage regulator operations decreased by about $10 \%$ and $20 \%$, respectively, for the TEC scenario compared to the Cloudy scenario. In addition, for the TE Challenge, all scenarios were simulated with the capacitor banks and voltage regulators in automatic control mode; as a result, the impact of only the TE controls cannot be discerned. In future work, we could perform simulations in which the voltage regulating device operations are pre-determined so that we can evaluate the impact of the TE controls only.

In our simulations, the power flow could not be solved for the PRD scenario after the TOU prices drop due to the load rebound's magnitude, so the simulation stopped at that time. This simulation fault illustrates that when house-level controls all respond to the same price signal, the load can respond in a synchronized way that can result in severe load steps and operational issues on a distribution feeder.

The proposed TEC approach issues active and reactive power incentive prices to individual homes based on their location. In this analysis, the average house energy cost is the same for the TEC scenario as for the Cloudy scenario. The TEC scenario has more outliers in energy cost, and a few houses have significantly higher or lower than the median cost. More extensive analysis and design are required to determine appropriate levels of compensation for PV curtailment and reactive power support that also considers revenue impacts for the utility. Future work is warranted to address how such unique prices can be applied while ensuring a socially acceptable level of fairness. Future work could also investigate the impact of issuing incentive signals to groups of homes that are connected to the same distribution transformer instead of to individual homes to reduce the communication requirements imposed by the proposed control approach.

We illustrated that NREL's co-simulation framework - the IESM-is consistent with the abstract component model that NIST proposed. This research also demonstrates that the IESM is able to simulate TE control approaches at a realistic feeder scale. We proposed and tested a networkaware voltage management TE control approach that had been simulated previously only with simpler power distribution networks, e.g., a 37-node system with 18 PV inverters and 375 air conditioners and batteries [9]. The TE Challenge scenario is a much larger system with more complex inverter and house appliance models, and this increased complexity exposed some challenges in the proposed approach. For example, the approach requires tuning the house-level and the network-level controllers - a challenging task, especially with data from a single day. Poor tuning can lead to slow response or oscillations. Tuning might be best performed via machine learning during a multi-month period and this is an area for future work. This example illustrates the value of complex system simulation in designing and evaluating transactive control approaches prior to field-testing.

The proposed control approach requires updates to incentive signals at a rate of 1-10 seconds and we selected to simulate an update every 10 seconds. Therefore, a simulation of a full day required 8,640 updates to the price signal, i.e., executions of the network-level controls. The PV inverter controls were also executed every 10 seconds. The air conditioner controls were executed less frequently, every 15 minutes. The air conditioner controls update asynchronously to avoid adverse impacts from all air conditioning loads making power adjustments at the same time based on the incentive signals, so only a few air conditioner controllers would update every time the network-level and PV inverter controllers updated. The controls are based on an online 
optimization approach, so each control update should take less than a second to execute. We found, however, that it took around 12 seconds to finish one 10-second simulation step, and therefore simulation of a full day for the TEC scenario took about 30 hours.

The long simulation duration made debugging issues with the simulation a slow process and was mitigated by launching several simulations with different options at the same time. The incentive signal calculation took 5-10 seconds, and the rest of the time was used for passing data between the different components of the IESM and waiting for GridLAB-D to move forward to the next time. We found that the incentive signal calculation time depends on the system size, i.e., the calculations take much longer for larger systems. We are working on algorithms to improve the efficiency of the distributed algorithm for controlling large networked systems. For example, a hierarchical, distributed algorithm for solving the same optimal power flow problem used in the TE challenge has been designed to significantly reduce the computational complexity without compromising accuracy. Numerical tests on a 11,000-node distribution system show a 33-fold improvement in the convergence speed [10].

\subsection{References}

[1] S. Mittal, M. Ruth, A. Pratt, M. Lunacek, D. Krishnamurthy, and W. Jones, "A System-ofSystems Approach for Integrating Energy Modeling and Simulation," Summer Computer Simulation Conference, Chicago, IL, July 26-29, 2015.

[2] A. Pratt, D. Krishnamurthy, M. Ruth, M. Lunacek and P. Vaynshenk, "Transactive Home Energy Management and Distribution Grid Impact," IEEE Electrification Magazine special issue on Smart Buildings and Transactive Energy, Dec 2016.

[3] A. Pratt, M. Ruth, D. Krishnamurthy, B. Sparn, et al., "Hardware-in-the-Loop Simulation of a Distribution System with Air Conditioners under Model Predictive Control," IEEE PES General Meeting, July 2017.

[4] B. Sparn, D. Krishnamurthy, A. Pratt, M. Ruth, and H. Wu, "Hardware-in-the-Loop (HIL) Simulations for Smart Grid Impact Studies,” IEEE PES General Meeting, August 2018.

[5] M. Ruth, A. Pratt, M. Lunacek, S. Mittal, W. Jones, and H. Wu, "Effects of Home Energy Management Systems on Distribution Utilities and Feeders Under Various Market Structures," 23rd International Conference on Electricity Distribution (CIRED), June 2015.

[6] X. Zhou, E. Dall'Anese, L. Chen, and A. Simonetto, “An Incentive-Based Online Optimization Framework for Distribution Grids," IEEE Transactions on Automatic Control, 63(7), 2019-2031, 2018.

[7] GridWise Architecture Council, GridWise Transactive Energy Framework version 1.0, Jan 2015.

[8] A. Bernstein and E. Dall'Anese, "Linear Power-Flow Models in Multiphase Distribution Networks," in the 7th IEEE International Conference on Innovative Smart Grid Technologies, Sept. 2017.

[9] X. Zhou, E. Dall'Anese, and L. Chen, "Online Stochastic Optimization of Networked Distributed Energy Resources,” arXiv preprint: 1711.09953, 2017.

[10] X Zhou, Z Liu, W Wang, C Zhao, F Ding, L Chen, "Hierarchical Distributed Voltage Regulation in Networked Autonomous Grids," arXiv preprint: 1809.08624, 2018. 


\title{
Section 4 TCS Team Report: Simulation of Dynamic Microgrid Based Operations in TE framework
}

\author{
Yogesh Bichpuriya, Narayanan Rajagopal \\ Tata Consultancy Services, India \\ Additional Team Members: Sumit Kumar Ray ${ }^{1}$, Subrata Indra ${ }^{1}$, Aashutosh Soni ${ }^{1}$, Subham \\ Kumar $^{1}$, Anupama Kowli ${ }^{2}$, Joel Jose ${ }^{2}$ \\ (1) Tata Consultancy Services \\ (2) IIT Bombay, DMC algorithm development
}

\begin{abstract}
TCS work focused on the use of microgrid reconfiguration to improve resiliency while maximizing use of renewables. The GridLab-D simulation environment was used for two research components, first coupled with an independent TE market model to study the TE Challenge scenario performance and second with a modified IEEE 8500 grid to study microgrid reconfiguration. For the first component, the report presents simulation results for the GridLAB-D grid model according to the TE Challenge scenarios: sunny weather, cloudy weather, and repeated cloudy simulation connected to a TE market simulation. Secondly, a Dynamic Microgrid Configurator software was used to analyze the grid continuously in preparation for any fault and then to sectionalize the grid optimally to maintain operation with minimum load curtailment. The research examines three fault conditions, looking at utilization of green power, reduction of losses, and percentage of load served for each case. In all cases green energy utilization increases when microgrids are sectioned, since the microgrids have only solar PV for power sources, while some loads may be curtailed in case of a fault on the substation feeder. Losses are reduced due to use of local power and elimination of external sources of power (with resulting distribution losses).
\end{abstract}

\section{Table of Contents}

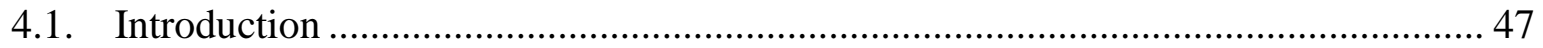

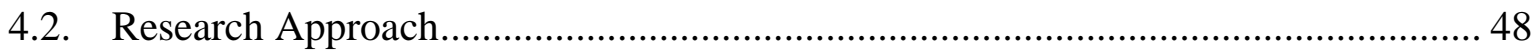

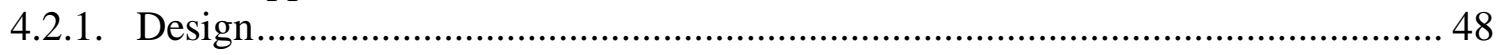

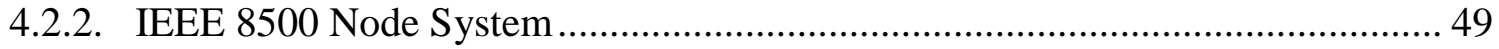

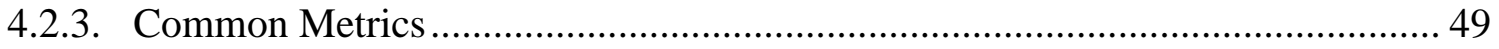

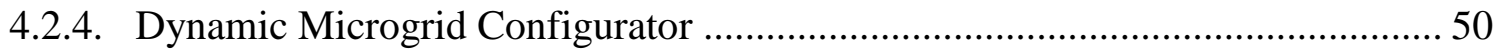

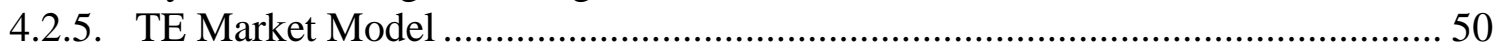

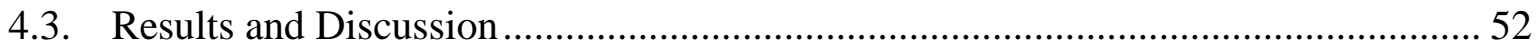

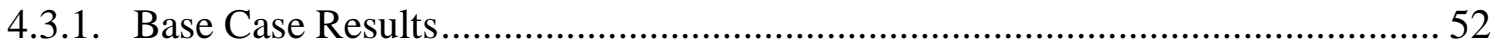

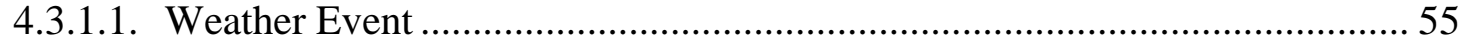

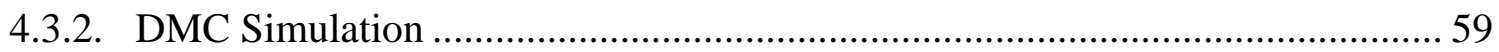

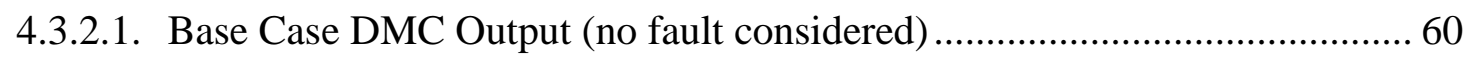

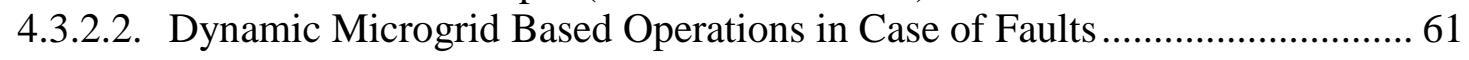

4.3.2.2.1. Case 1: Fault or Contingency Causing Outage of the Substation............61

4.3.2.2.2. Case 2: Fault on Line Between Two Microgrids ..................................62

4.3.2.2.3. Case 3: Fault Within a Microgrid ......................................................63

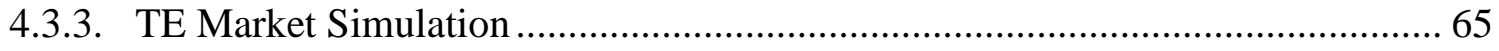

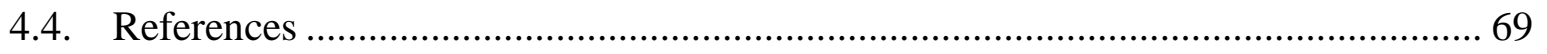




\subsection{Introduction}

The intermittency of renewable energy sources makes it very difficult to maintain the supplydemand balance. A TE Framework utilizes the flexibility of demand by sending a transactive signal - taking into consideration willingness and preferences of prosumers to set price signals.

As an economic construct, this entire ecosystem can be thought of as a retail electricity market where participants submit their demand and supply bids. The distribution system operator (DSO) will match these bids to clear the market and find a market-clearing price. Each participant can then compare this market-clearing price with its bid price and act accordingly. These participants could be prosumers or devices. With millions participating in a distribution grid, it is important to have a scalable TE model, smart devices, and sophisticated control systems to run the system within technical limits.

To provide reliable and resilient network operations, TCS has conceptualized a dynamic microgrid configurator (DMC). DMCs configure the distribution network into multiple irregularly honeycombed microgrids to meet variable electrical load demands. These flexible structures can be created based on the network topology, line power flows, weather, demand and distributed energy resources.

During normal operations, the DMC will periodically run an algorithm to configure these dynamic microgrids (DMs) through network topology processing and distribution power flow analysis. Current DM configurations will be readily available to the DSOs, and the DMs will continue to operate as one interconnected network. Islanding options will be exercised only when contingencies or events such as faults occur.

By continuously redefining microgrid boundaries, it will be easier to transition to an islanded mode during faults and return to interconnected mode when the network resumes normal operations. These seamless transitions are supported by distribution automation infrastructure, such as intelligent switches or reclosers, with communication capabilities - both hierarchical and peer-to-peer.

DMs provide additional degrees of freedom to the DSO to address critical issues, which may arise from temporal variability of renewable sources and penetration of electric vehicles, resulting in a dynamic shift of point of connection of source and sink nodes.

Increased DER (solar PV with battery, wind, etc.) penetration will ensure continuous supply in case of islanded microgrids so that consumers may experience uninterrupted power under any grid conditions with dynamic microgrid based operations (DMBO). We can measure DMBO's performance by evaluating it against a defined set of key performance indices, e.g., green power utilization, load served, and system losses tied to distribution system efficiency, load balance and more. However, the willingness and preferences of the DER providers must also be taken into consideration.

The DMC-TE co-simulation platform will be capable of responding to the changes in grid conditions to create dynamic microgrid groups, which satisfy the economic and control mechanisms of a TE system. However, the results from this combined DMC-TE co-simulation work are not available for this report. 
The co-simulation system is comprised of the following subsystems.

1. Distribution system simulation in GridLAB-D.

2. Dynamic Microgrid Configurator (DMC) - Microgrid configurator tool that provides network configurations.

3. Double-sided auction model for TE developed exclusively for this simulation.

The co-simulation uses a modified version of the IEEE 8500 node system.

\subsection{Research Approach}

\subsubsection{Design}

We started with the objective to develop a simulation model for dynamic microgrids with a TE market. The design approach can be broadly summarized into the following steps.

- Development of a DMC algorithm for a distribution network with balanced three phase power.

- Power flow studies using GridLAB-D for the IEEE 8500 network under Baseline, Sunny and Cloudy weather scenarios.

- Testing of the DMC algorithm on the three-phase equivalent of the IEEE 8500 node system. The DMC model takes the network state (power flows, demand, supply and network connectivity information) to determine the dynamic boundaries of the microgrids. The current model for DMC does not consider any pricing model.

- Development of a TE double-auction market model that considers the bids of all participants (prosumers) and the network connection information. It results in a simplified market model for which the technical parameters (i.e., network capacity, voltage etc.) are validated with GridLAB-D.

The details of the DMC and TE models are in the following sections. While DMC helps in improving resiliency with dynamically configuring the network, an assumption made while making honeycombed microgrids is that we can utilize/control whatever DERs are available. This may not be possible without any economic/TE signal being sent to the DER providers to dynamically balance demand and supply. So, the original intention in the co-simulation is to provide TE signal to DER providers to minimize load curtailment or maximize green energy utilization while maintaining the resiliency with the help of DMC. Combining the DMC and TE models is a work in progress, with the remaining goal to develop a co-simulation for dynamic microgrid with TE market.

The scenarios are to be simulated in the following way.

1. Baseline scenario: The distribution network is provided without any constraints of weather or market participants. A simulation on this network is carried out to conduct the power flow analysis.

2. Addition of Weather scenario to the Baseline: The operation of DER (i.e. Solar) depends on the weather. Solar PV generation could suddenly be decreased by cloud cover. Simulation is carried out to check the effect of such a scenario and test the power flows.

3. Addition of DMC, DMBO: The DMC will change the network configuration and create self-balanced regions which will serve the maximum number of customers and also maximize the green energy utilization. 
4. TE Market model addition: A market agent takes bids from the participants, i.e. demand (house appliances) and supply (generators), and clears the market. The participants' bids are aggregated at the three-phase nodes. This could be considered equivalent to a scenario where aggregators consolidate the bids of their subscriber base of consumers and prosumers. Power flow analysis is carried out after addition of the TE market.

\subsubsection{IEEE 8500 Node System}

The IEEE 8500 network has unbalanced nodes and consists of three-phase and single-phase nodes. For the DMC research, it is modified by aggregating the loads at single-phase nodes up to the three-phase nodes to perform the simulation of various scenarios, assuming a balanced network. The parameters recorded at node or house levels are aggregated to the three-phase node level. These loads and solar PV are aggregated to form 649 three phase nodes. The truncated network representation is shown in Figure TCS-1.

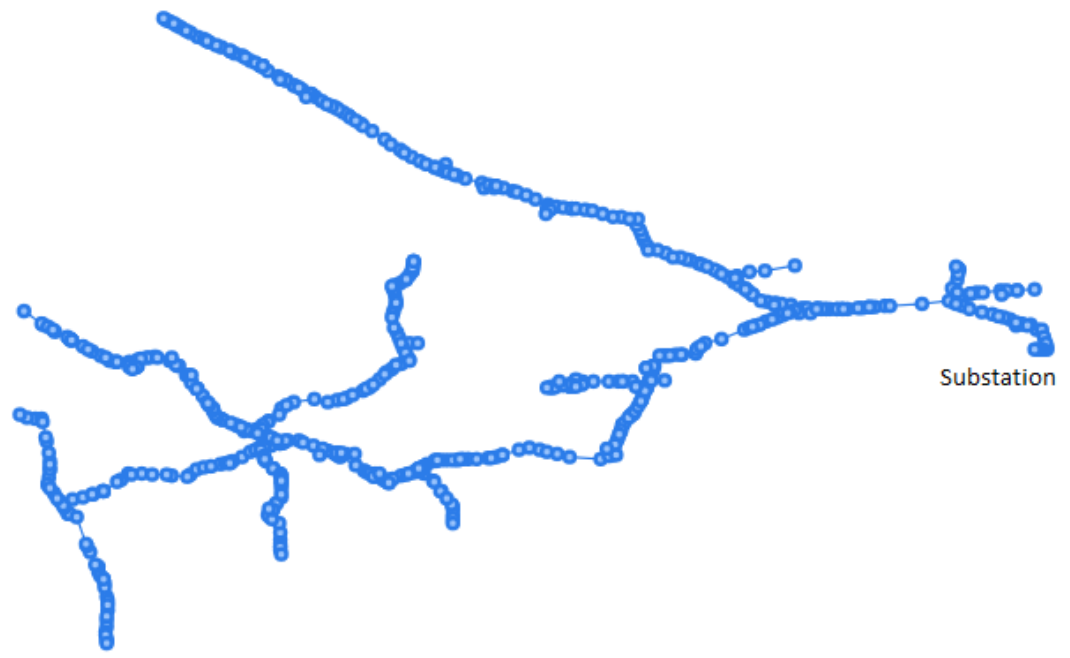

Figure TCS-1 Modified network of 8500 feeder system, truncated to three-phase nodes only

\subsubsection{Common Metrics}

Common metrics are the parameters to report and analyze after each simulation. The selected metrics are given in Table TCS-1.

Table TCS-1 Common Metrics

\begin{tabular}{|c|c|}
\hline Metrics & Description \\
\hline Economic & $\begin{array}{l}\text { Market clearing price at each meter, quantity } \\
\text { cleared }\end{array}$ \\
\hline Utility & Real and reactive power, energy and loss \\
\hline Capacitor and regulator operation & Count of control actuations \\
\hline Electric parameters & $\begin{array}{l}\text { Voltage (L-N and L-L), Voltage imbalance, } \\
\text { Total house load, HVAC load, Total water } \\
\text { heater load, Solar power, House air temp and } \\
\text { its deviation, Total bill }\end{array}$ \\
\hline Green energy utilization & $\begin{array}{l}\text { Ratio of renewable energy generation over total } \\
\text { energy consumption }\end{array}$ \\
\hline
\end{tabular}




\subsubsection{Dynamic Microgrid Configurator}

During faults and contingencies all or some customers in the distribution system will face power failure. The DMC helps in using DERs to supply the customers during such events, by clustering customers and DERs into multiple microgrids. These microgrids are formed by dynamically changing boundaries depending on availability of distributed generation and demand to be served. The interconnected microgrids are nearly supply adequate, enabling successful islanded operation when required. Thus, DMC reduces the number of customers that face interruption, improves reliability, and increases the utilization of DERs.

The dynamic microgrid algorithm consists of a $k$-way spectral partitioning algorithm [1]. The algorithm divides the network graph into $\mathrm{k}$ clusters which meet the supply and demand optimally. Each cluster consists of at least one generation and load. The inputs to the DMC algorithm are: (1) network topology, (2) power flows on lines, and (3) demand and generation profile.

Figure TCS-2 shows the block diagram of the conceptualized dynamic microgrid operations with a TE framework. The DMC algorithm divides the network into clusters/microgrids depending on the power flow results of the instant load and generation which depend on the weather (sunny/cloudy) scenario and network parameters. As seen from the figure, DMC uses information about load, DER schedules, network constraints, real time network events like outages, distribution network model, etc. for creating honeycombed microgrids at each interval. Information on various scenarios, like grid failure and microgrid faults from past historical data/events, are stored in a repository which can be used by the DMC when a similar event occurs. The interfaces to a TE framework are being developed.

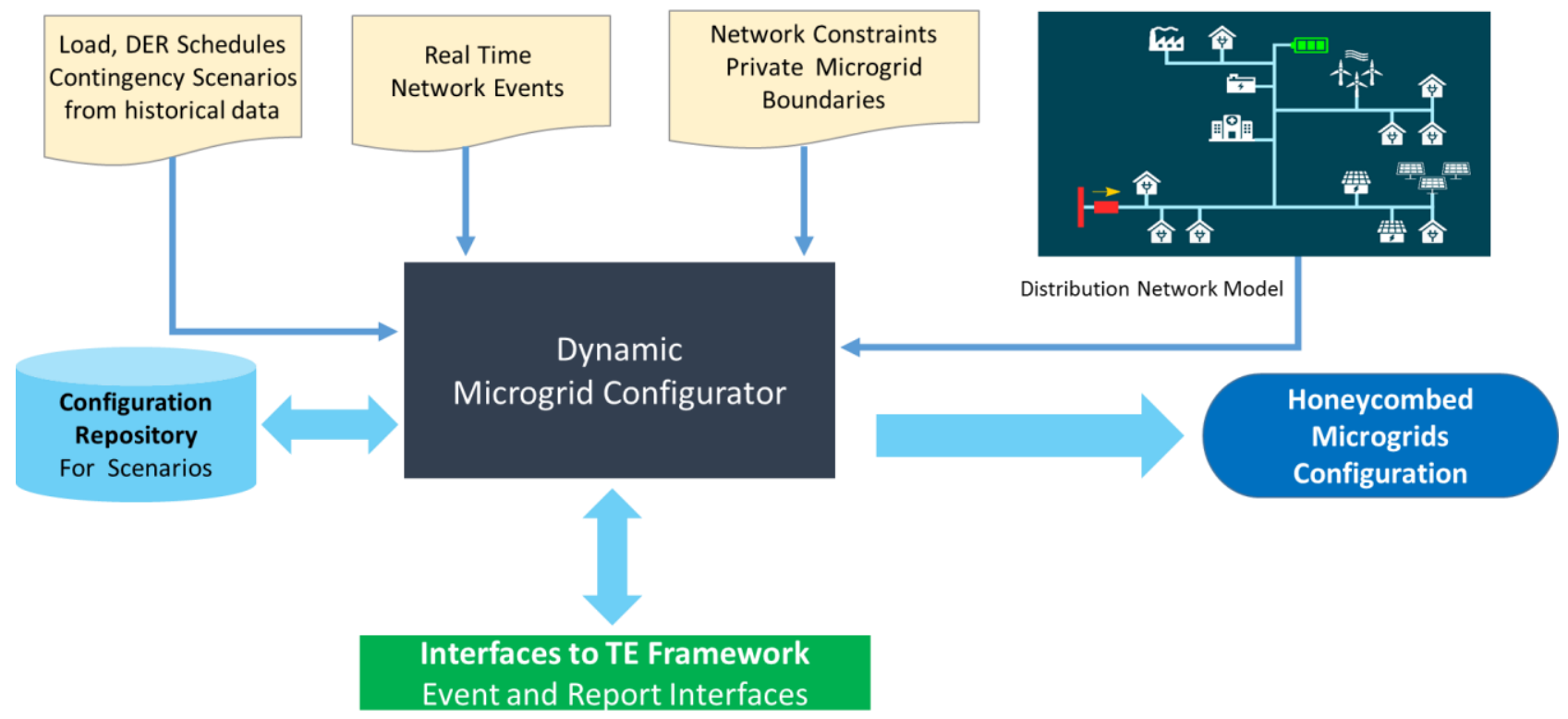

Figure TCS-2 Dynamic microgrid operations

\subsubsection{TE Market Model}

The market model used here is a double-auction market wherein both sides, demand and supply, participate and place their bids and offers respectively. These bids are in the form of a PriceQuantity pair bid for each time interval. From the demand side, HVAC loads place bids in the 
market. However, any kind of flexible load can participate in the market. The market runs every five minutes, hence HVAC loads and generators bid for demand and supply respectively for the next five-minute interval. The bids of HVAC loads depend on customer requirements and comfort, weather, etc. whereas supply offers depend on availability of generation. The demand side consists of the transactive loads in the houses at the distribution end. The supply side includes the substation input and the solar PV present at the houses. The cleared price from the market model works as the input to the network model as the transactive signal. This overall simulation is described in Figure TCS-3.

In the market simulation, a double-auction market is simulated external to the GridLAB-D network model. The bids of each participant and network information are read from GridLAB-D and then used for simulating the market model which gives the market cleared price (MCP) and market cleared quantity (MCQ) of each participant. The MCP is then used to decide the state of HVAC units in each house. If the MCP <= bid price, the bid quantity has been cleared, which means the HVAC can be switched on. If $\mathrm{MCP}>=$ bid price, the bid quantity is not cleared, which means the HVAC unit needs to be off. This state (on/off) of every HVAC unit is then written into the GridLab-D file and then a power flow simulation is run to check the electrical parameters of the network.

The above approach helps in reducing the computing effort involved in distributed locational marginal price (DLMP) models where network and market simulation are done simultaneously. Our model is based on the parallel simulation of power flow and market clearing, which may require iteration if the market clearing results violate any limits on technical parameters. However, for a distribution network with millions of TE participants, the simplified approach may help in reducing computational time.

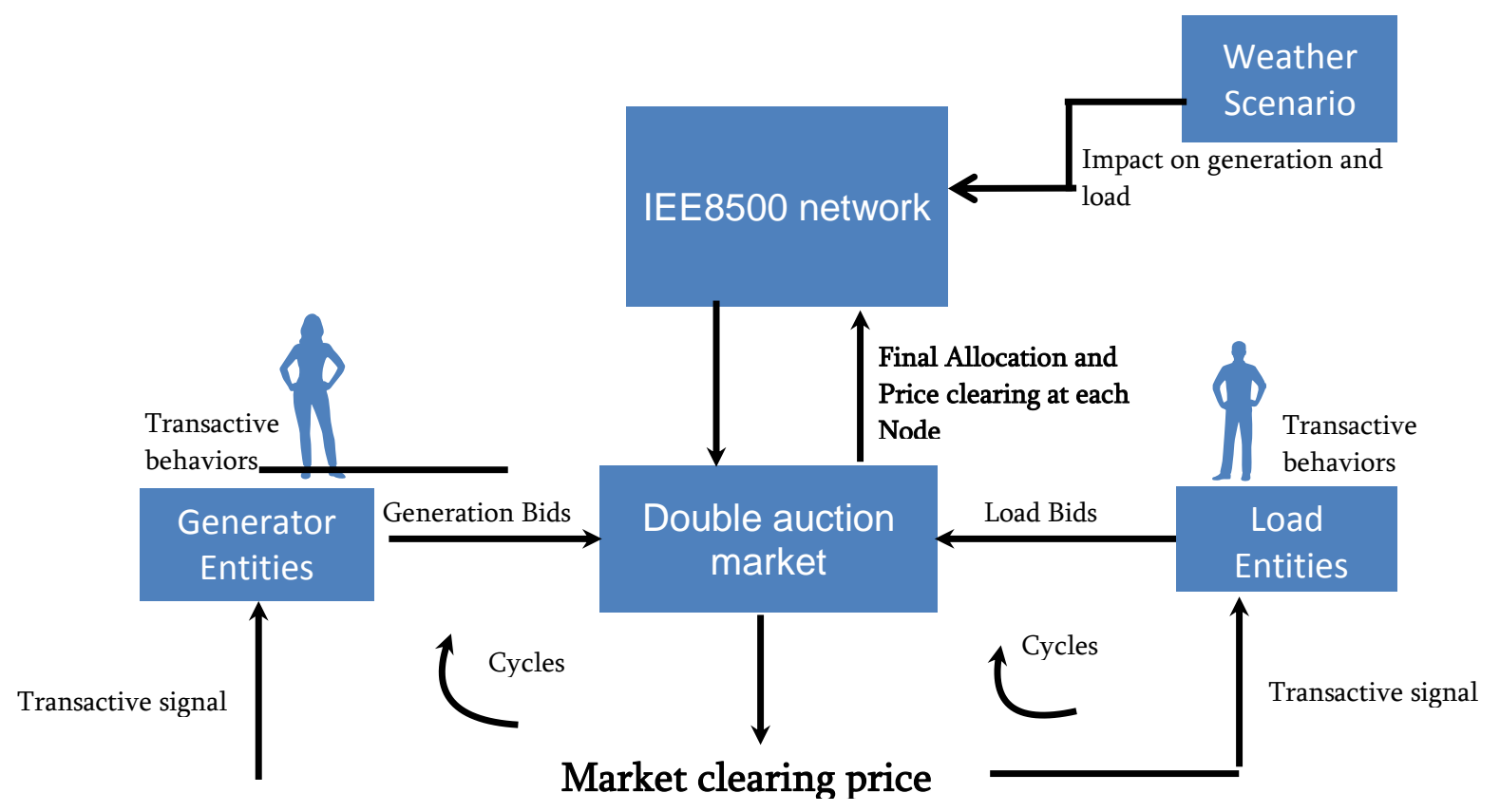

Figure TCS-3 Transactive energy market model 


\subsection{Results and Discussion}

\subsubsection{Base Case Results}

The base case includes the basic simulation of the IEEE 8500 network and power flow analysis of the same. This is performed without any market operation in the network. This will help in comparing the effect of additional scenarios added on top of the base scenario, like microgrid configurator and market module.

The outputs of the base case are shown in Figure TCS-4 to Figure TCS-8. From Figure TCS-4, it can be clearly observed that the substation power decreases when there is sunlight, as solar PV supplies the load during that time. House load is more than HVAC load as most of the houses consist of fixed loads along with HVAC loads. The minimum, maximum and average temperatures of all houses are plotted in Figure TCS-6, which shows the temperature of all houses within the range of $20{ }^{\circ} \mathrm{C}$ to $30^{\circ} \mathrm{C}\left(68^{\circ} \mathrm{F}\right.$ to $\left.86^{\circ} \mathrm{F}\right)$. Capacitor switching is shown in Figure TCS-7 which takes place due to power requirements or voltage compensation. Similarly, voltage regulators are switched on when voltage compensation is required as shown in Figure TCS-8.

The base case scenario is simulated on the IEEE 8500 system with single-phase low voltage lines included without truncation and aggregation.

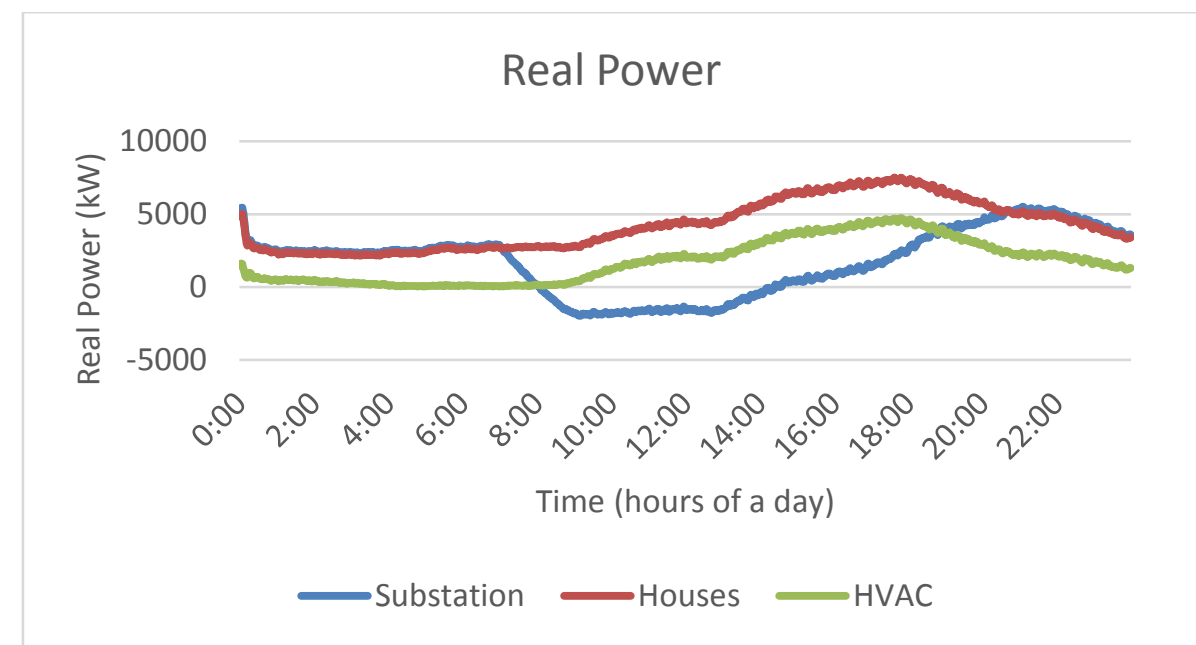

Figure TCS-4 Real power (Substation, House load and HVAC load) on a sunny day 


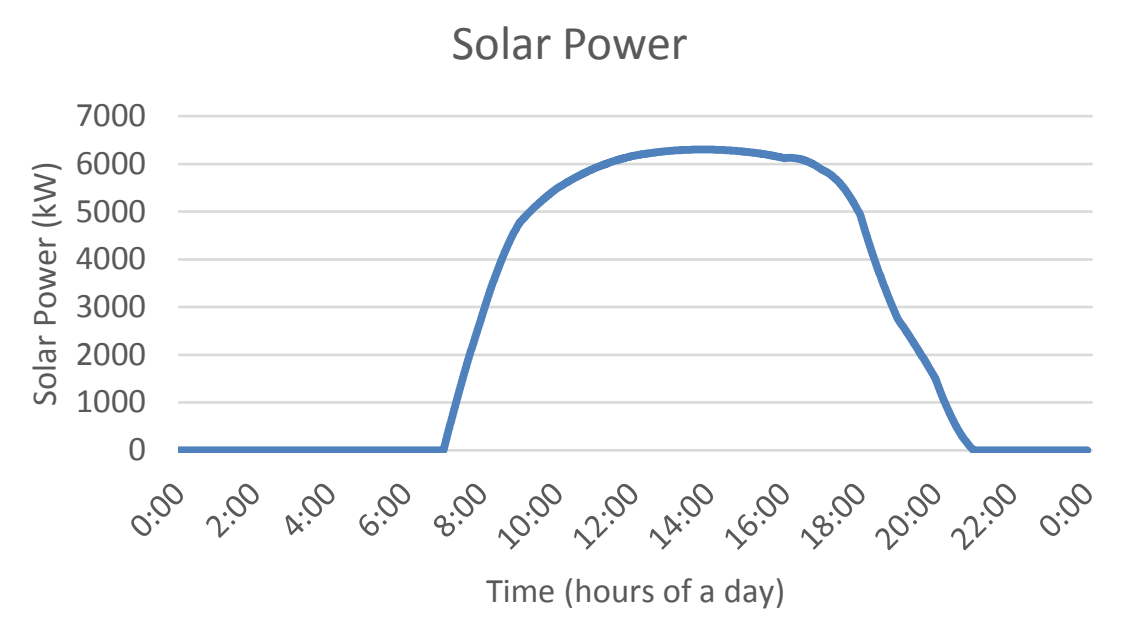

Figure TCS-5 Solar PV power on a sunny day

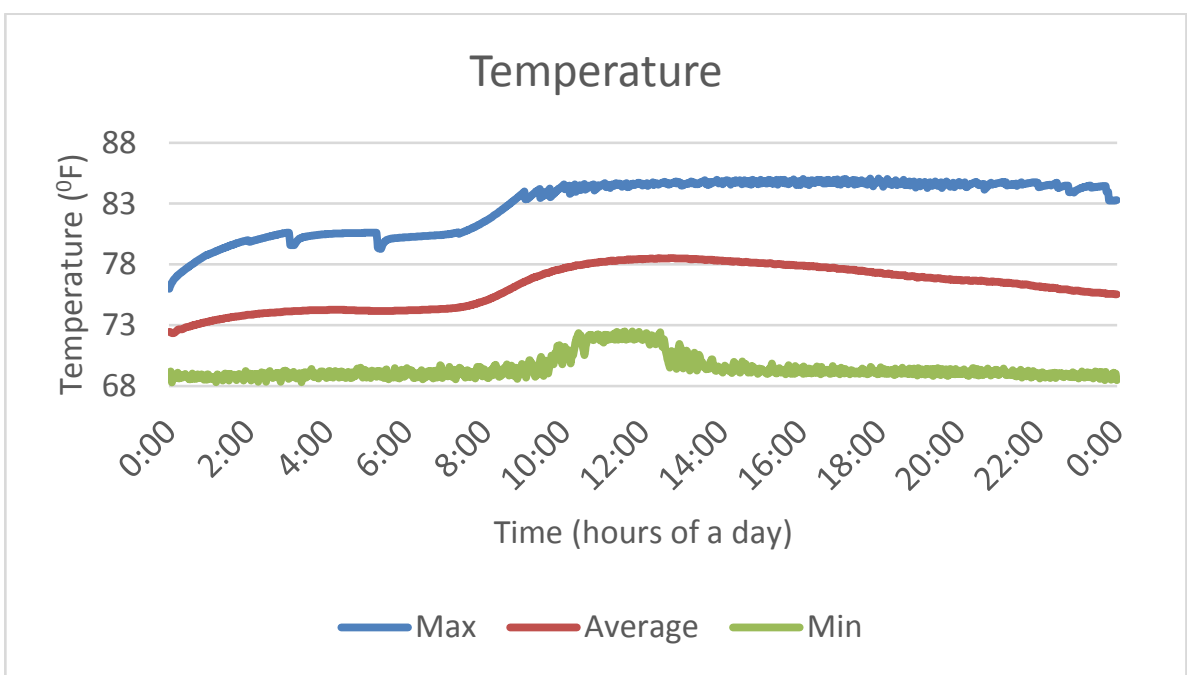

Figure TCS-6 Max, Min and Average temperature of houses on a sunny day

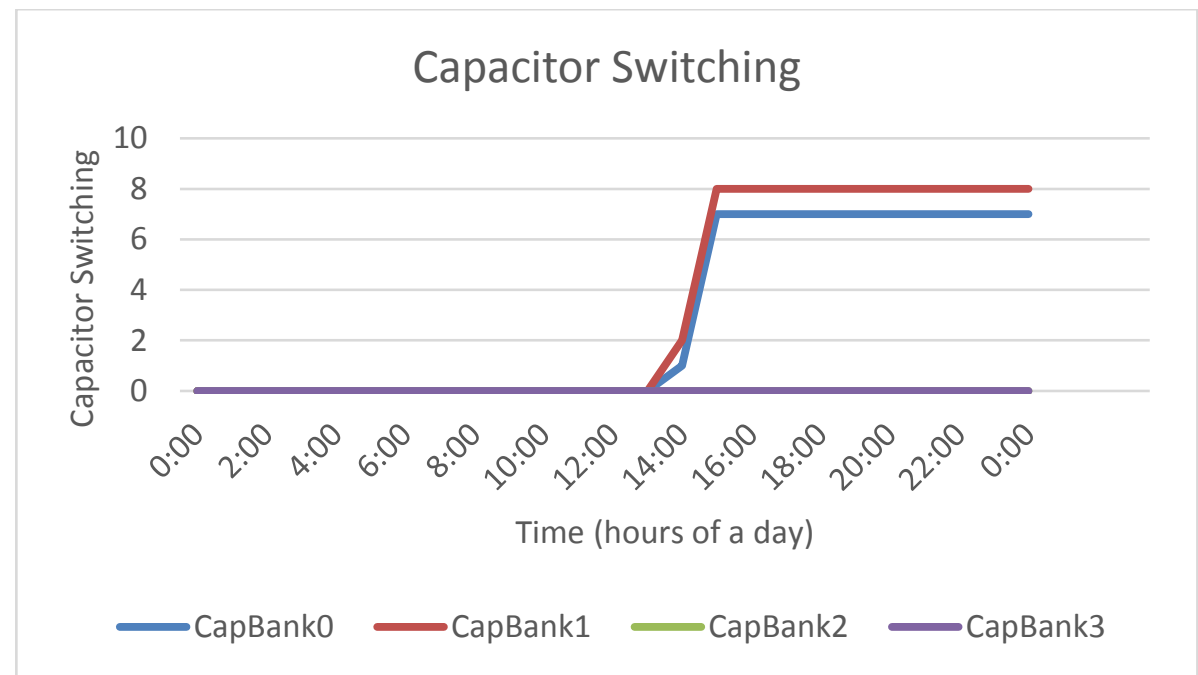

Figure TCS-7 Capacitor switching for base case on a sunny day 


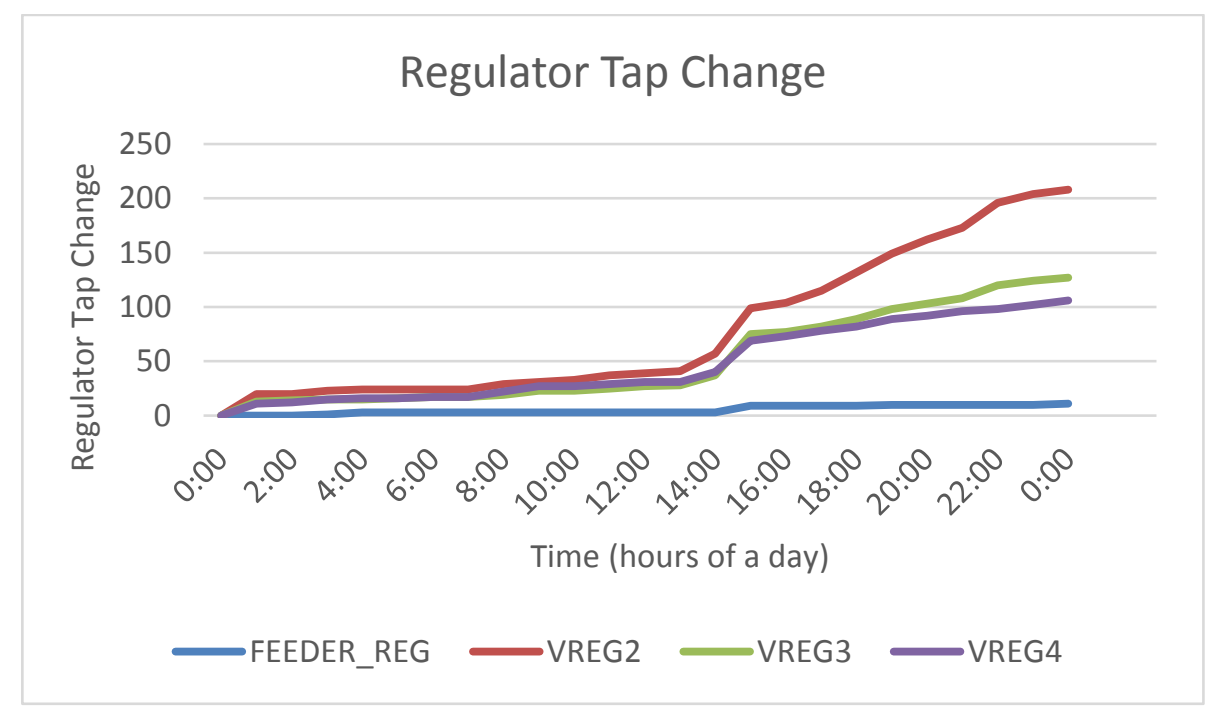

Figure TCS-8 Regulator tap change for base case on a sunny day

The base network of the 8500 bus system is simulated with sunny weather to get the required power flow. The power flow results are shown in Figure TCS-9 and Figure TCS-10 below. Figure TCS-9 shows the unbalanced phase voltages of the main substation node, which are well within tolerance limits (+/- $5 \%$ ) from the nominal voltage of 7,500 V.

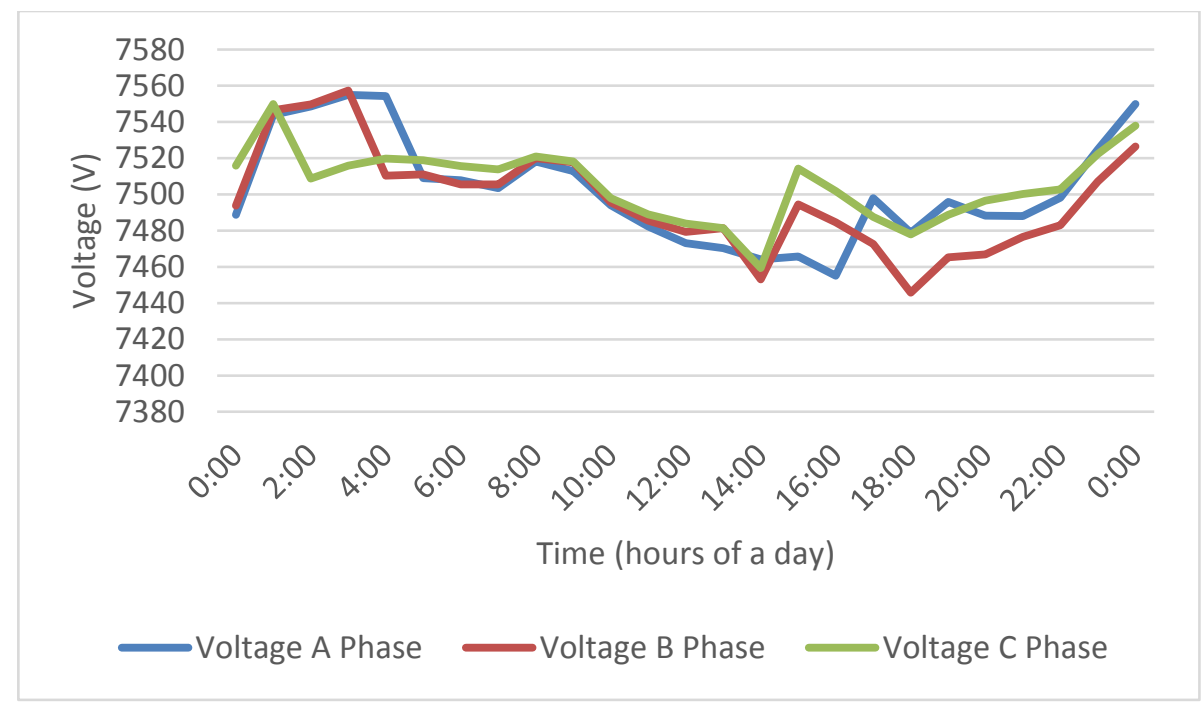

Figure TCS-9 Voltage Profile of the Substation nodes 


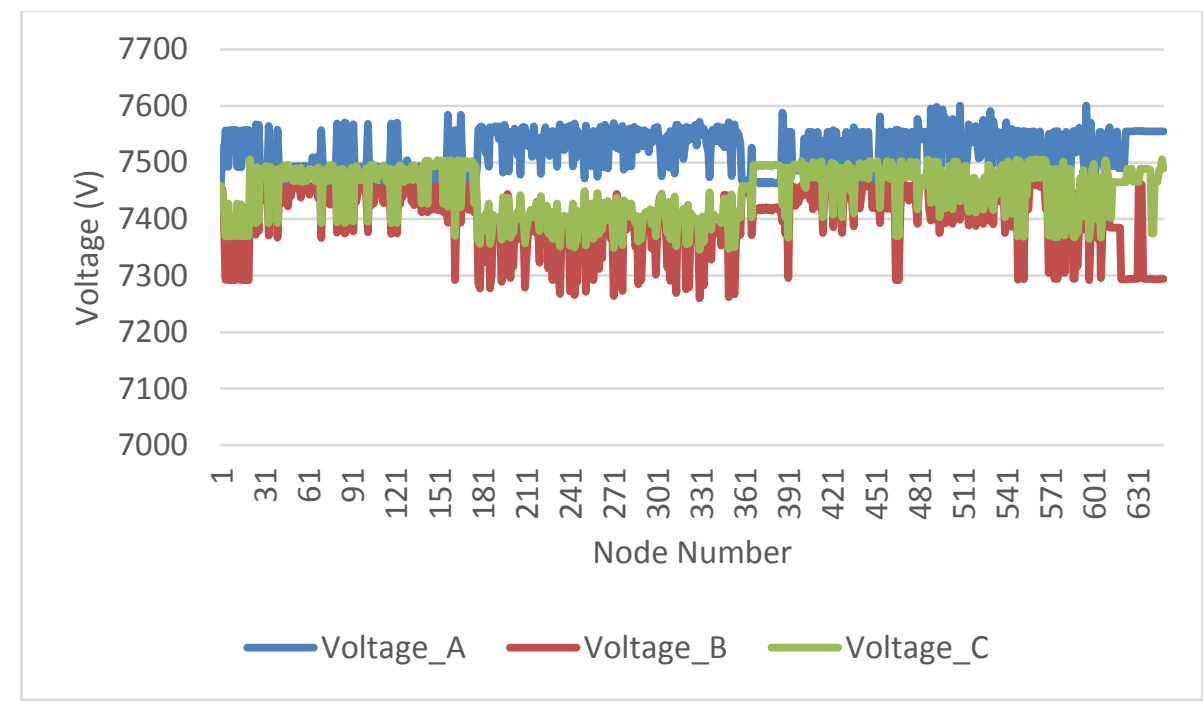

Figure TCS-10 Voltage at each node at 15:00

The unbalance in the system can be observed clearly from the voltage plots of Figure TCS-10. The power loss in each three-phase line is shown in Figure TCS-11. Power loss is observed to vary from around less than 1 watt to 900 watts. These variations are due to the difference in power flow, capacity of the lines, variation in their lengths, and losses in components such as the feeders and transformers connecting them to the three-phase nodes, etc.

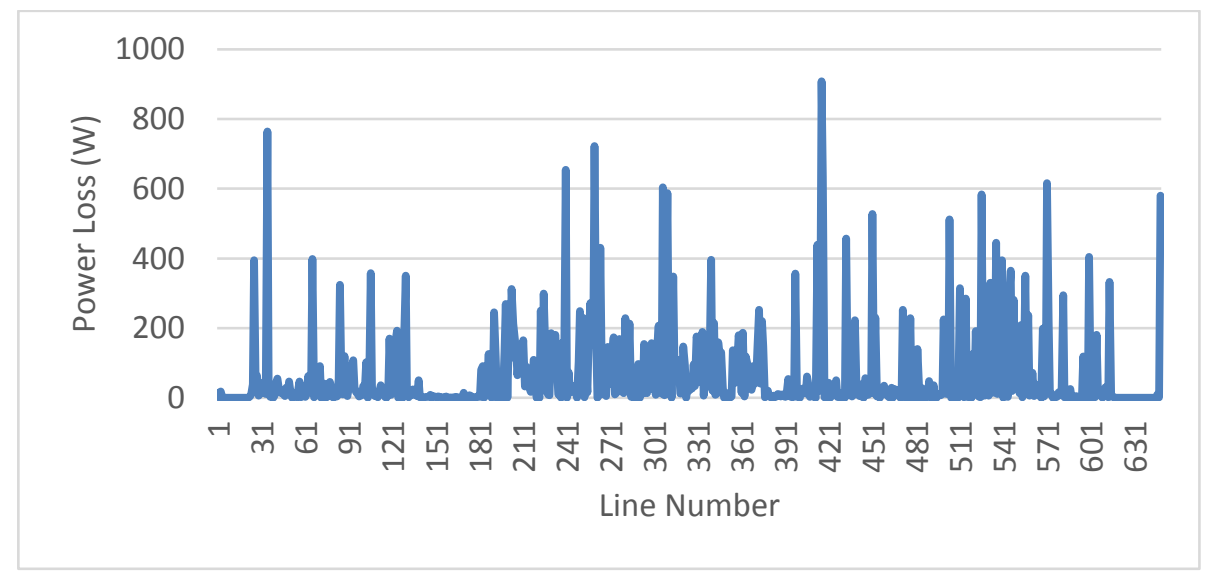

Figure TCS-11 Line Power Loss in 8500 Network

\subsubsection{Weather Event}

The weather event, with cloud cover occurring for around 2 hours from 2:30 p.m. to 4:30 p.m., decreases the solar PV power generation. The results with cloudy weather scenario are shown in Figure TCS-12 to Figure TCS-16.

From Figure TCS-12, it can be clearly observed that the substation power decreases when there is sunlight, as solar PV supplies the demand during that time. When sudden cloud cover reduces the solar output, substation power increases for that duration. The house load is more than the HVAC load as most of the houses consist of fixed loads along with HVAC loads. 
Minimum, maximum and average temperature of all houses are plotted in Figure TCS-14, which shows the average temperature of all houses within the range of $23{ }^{\circ} \mathrm{C}$ to $26{ }^{\circ} \mathrm{C}\left(73{ }^{\circ} \mathrm{F}\right.$ to $\left.78^{\circ} \mathrm{F}\right)$. The variation in the temperature of houses during the day depends on weather and customer comfort. From Figure TCS-15, it is observed that capacitor bank 2 is switched on, while in a sunny scenario as shown in Figure TCS-7, capacitor bank 2 is off. This may be due to the requirement of additional power and voltage compensation in the cloudy case. Similarly, voltage regulators are also switched on at instances where voltage compensation is required, as shown in Figure TCS-16.

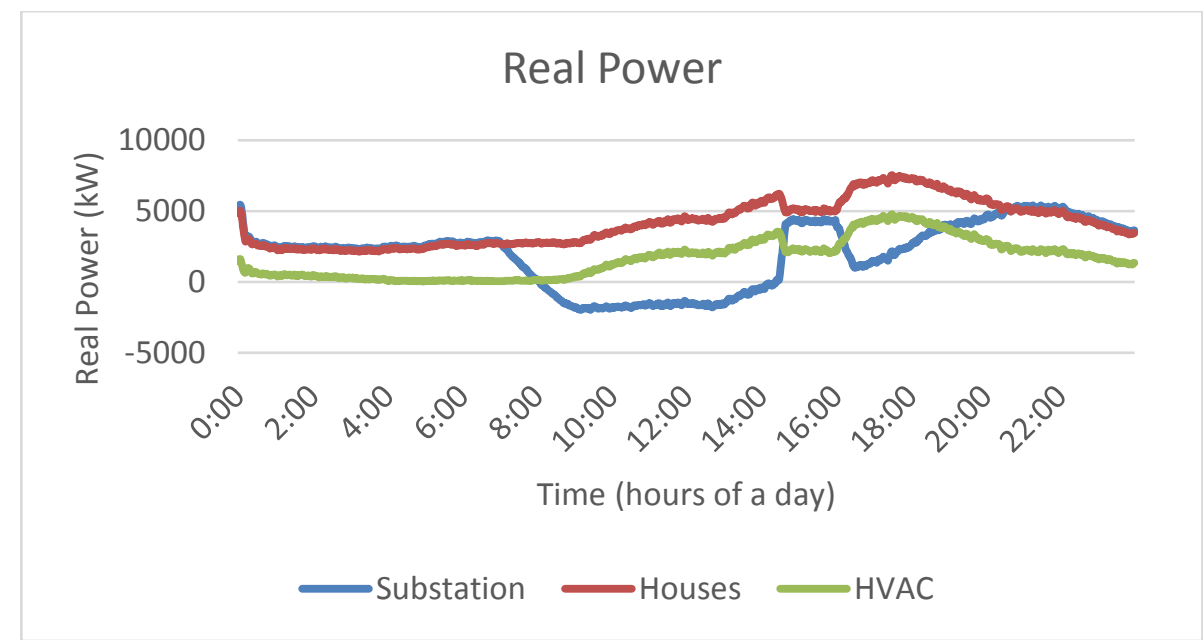

Figure TCS-12 Real power (Substation, House load and HVAC) on a cloudy day

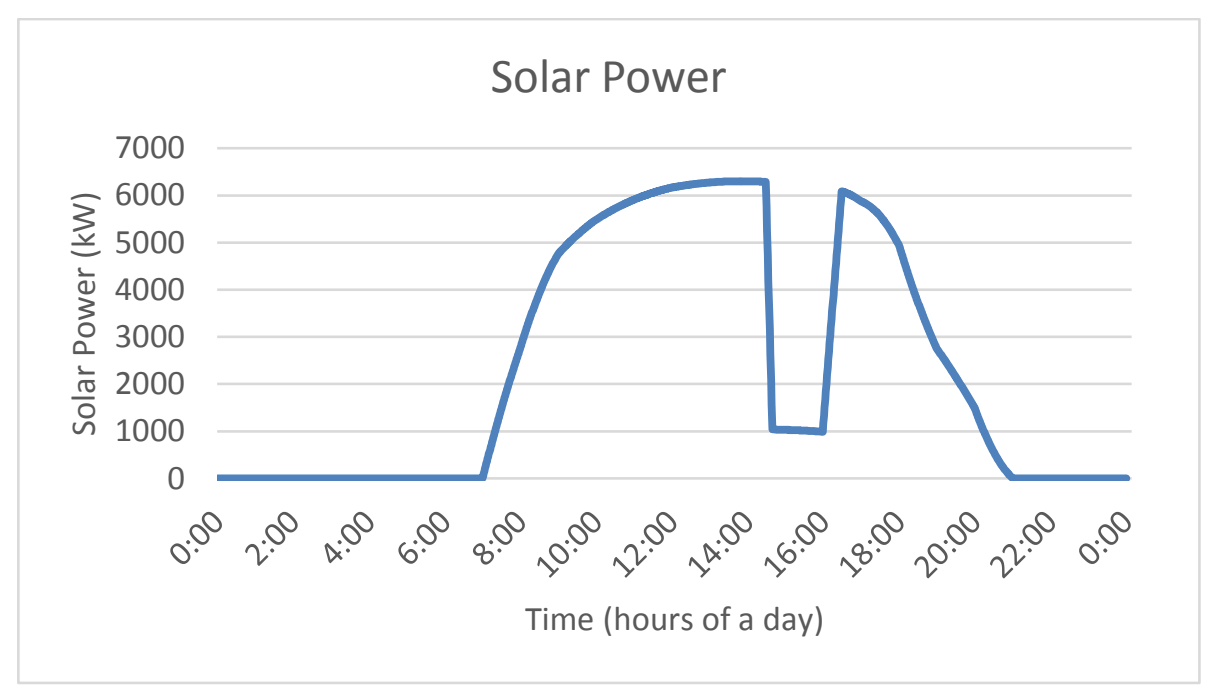

Figure TCS-13 Solar PV power on a cloudy day 


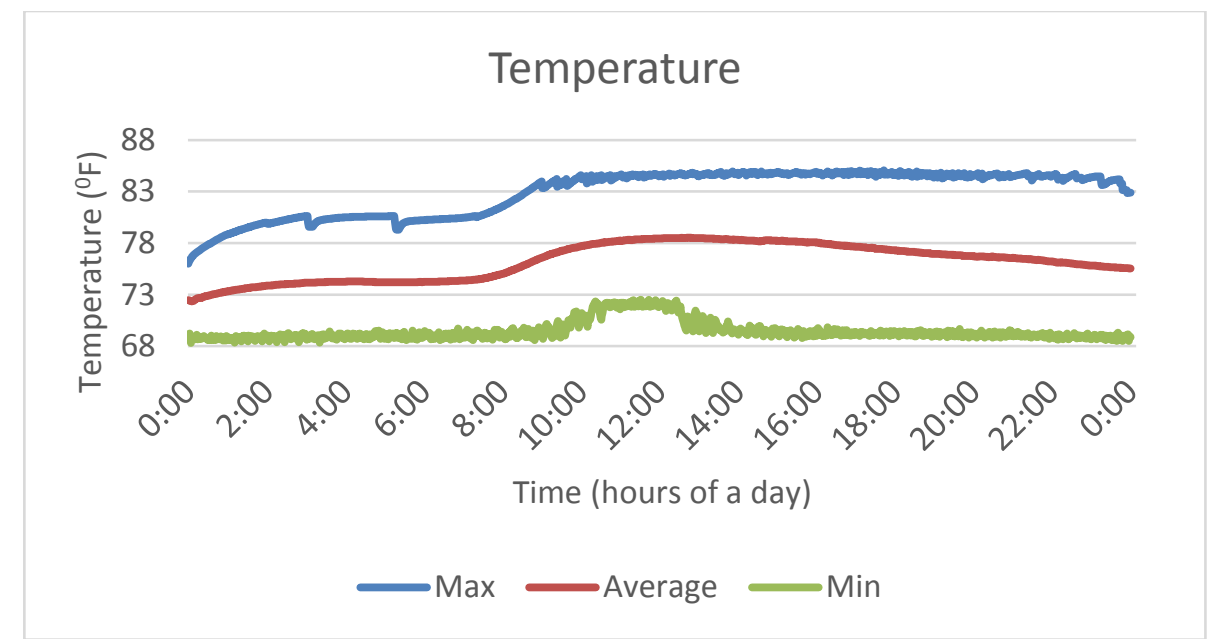

Figure TCS-14 Max, Min and Average house temperatures on a cloudy day

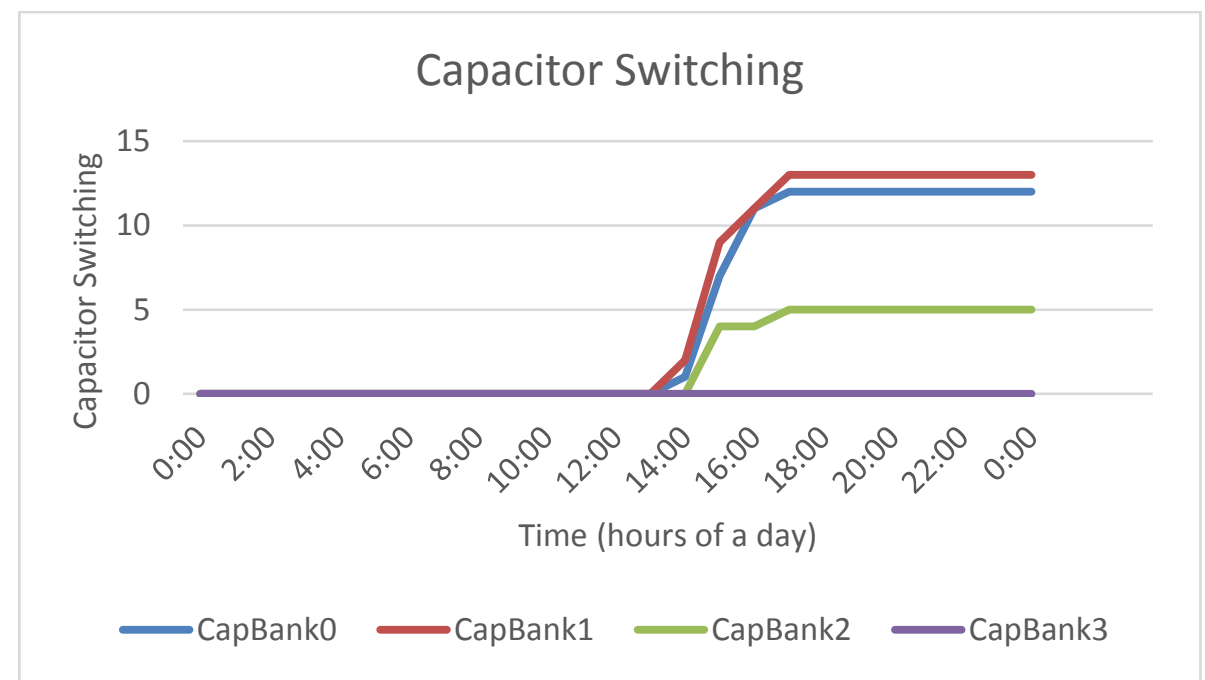

Figure TCS-15 Capacitor switching for base case on a cloudy day

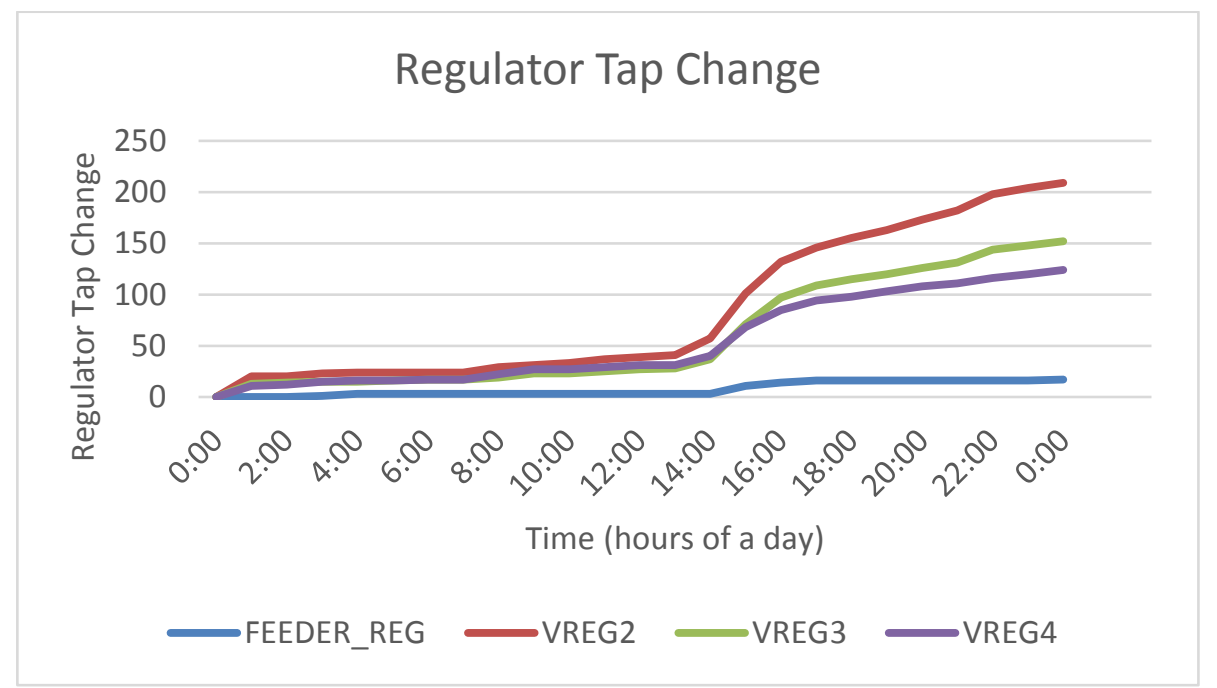

Figure TCS-16 Regulator tap change for base case on a cloudy day 
The IEEE 8500 node system is simulated with cloudy weather to get the required power flow. The power flow results are shown below in Figure TCS-17 and Figure TCS-18.

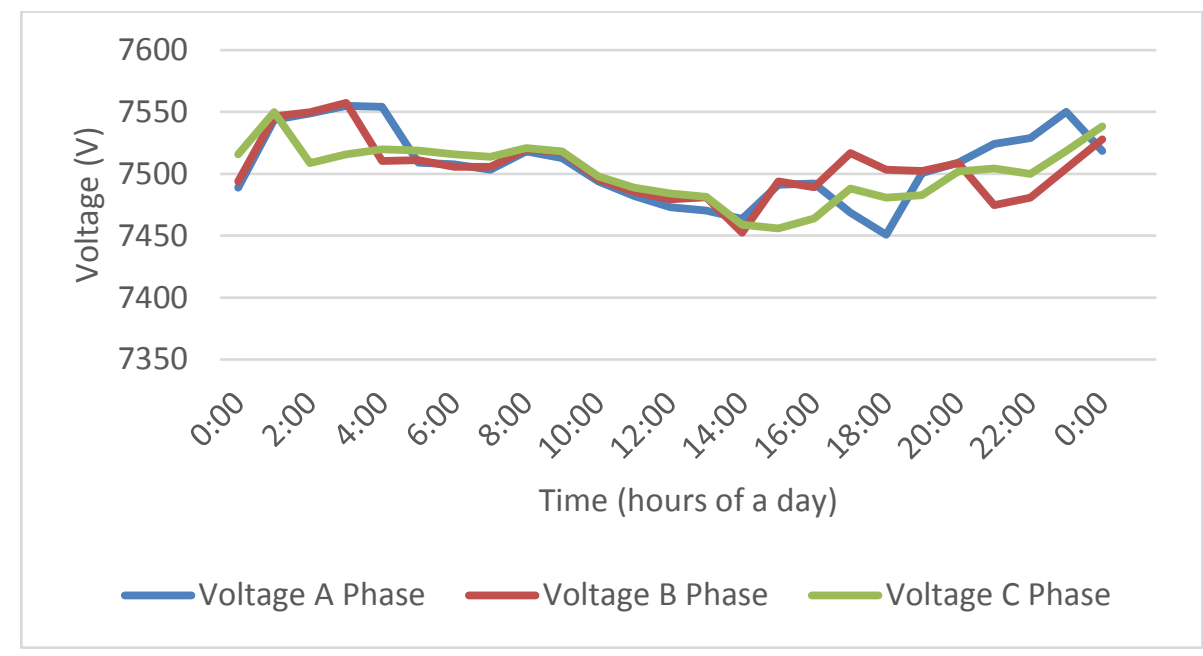

Figure TCS-17 Voltage profile of HVMV_Sub node

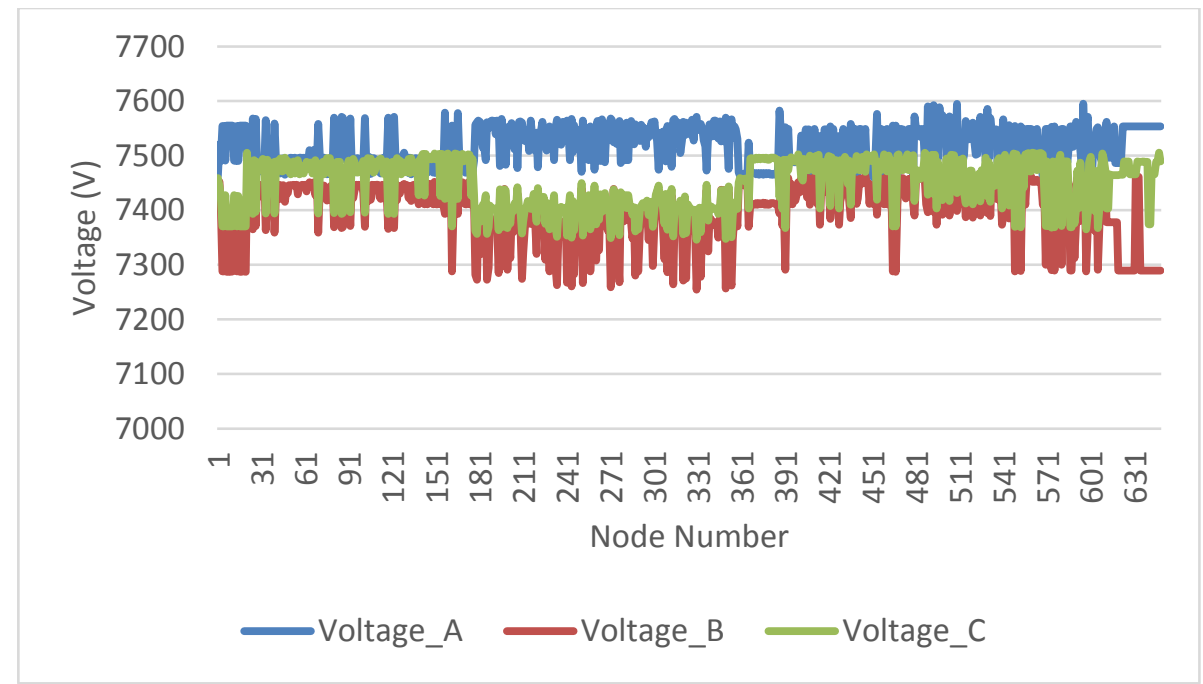

Figure TCS-18 Voltage at each node at 3 PM

The unbalanced 8500 system is simulated, and the results are shown in the above figures for three phase nodes and lines. As seen in Figure TCS-17 and Figure TCS-18, voltages at each bus are within tolerance limits (+/- $5 \%$ ) from the nominal voltage of $7500 \mathrm{~V}$. The unbalance in the system can be observed clearly from voltage plots of Figure TCS-18.

In the cloudy weather condition, more power has to be drawn from the grid to meet the demand as solar generation decreases, due to lower solar irradiance. The increase in power drawn from the grid in cloudy conditions is shown in Figure TCS-19 and Figure TCS-20. Figure TCS-19 shows real and reactive power supplied by the substation bus in case of cloudy weather. Figure TCS-20 is the active power drawn from the substation node in both sunny and cloudy scenarios. 


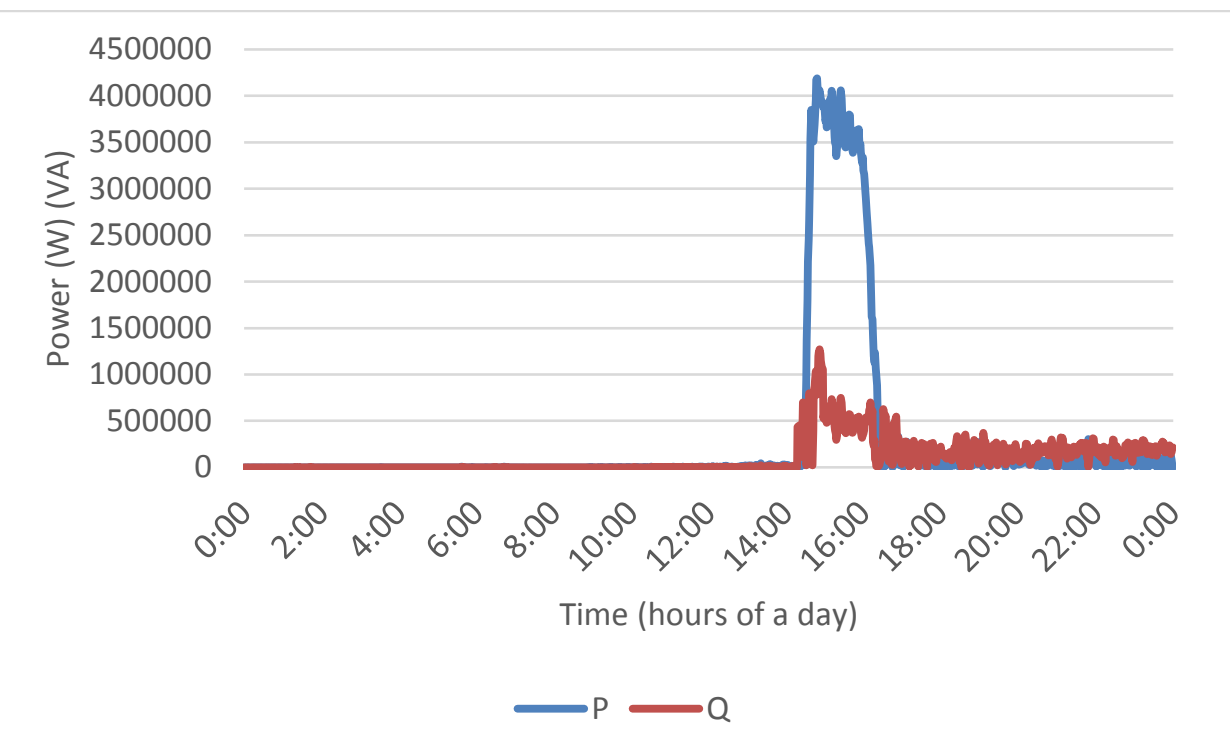

Figure TCS-19 Increase in Power drawn from grid during cloudy weather scenario

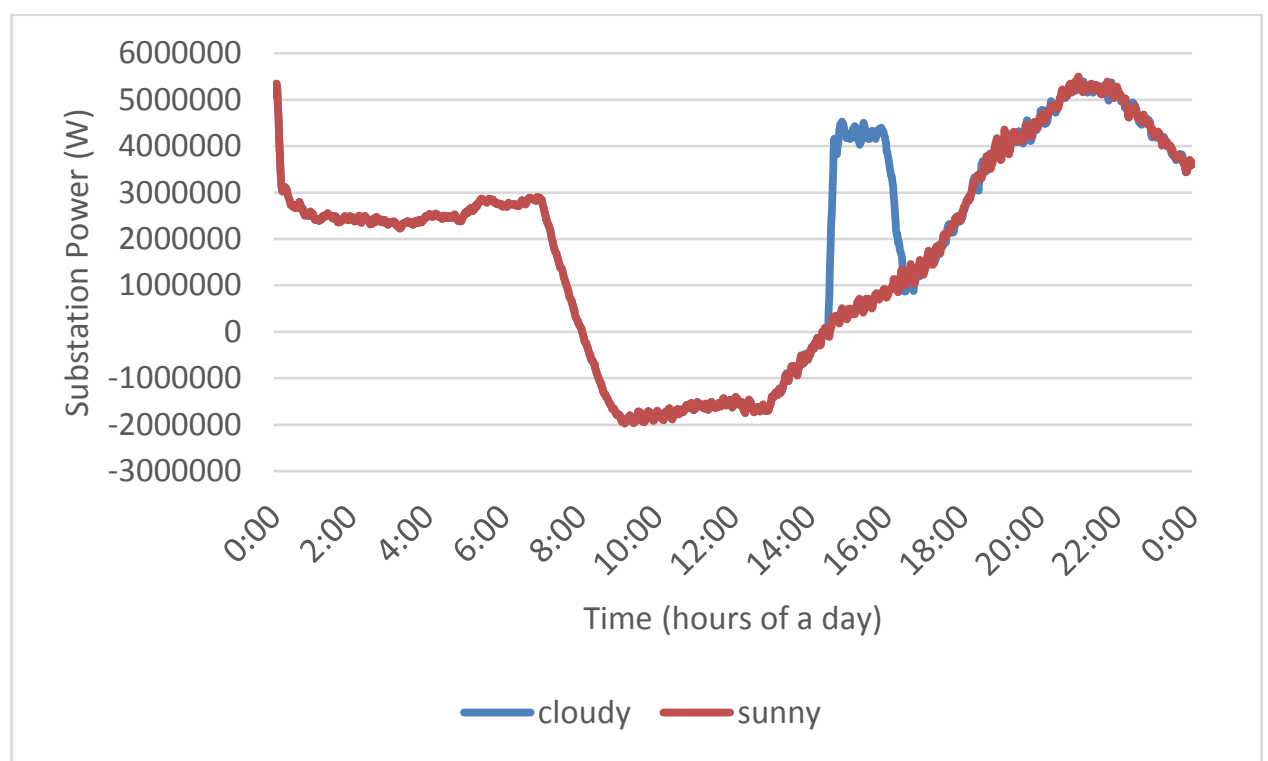

Figure TCS-20 Substation real power of aggregated 8500 network

\subsubsection{DMC Simulation}

The configuration of the entire network into microgrids is based on power flows and network topology at a particular instance, such that each microgrid is self-sufficient with generation from DERs present in that microgrid, making the system more reliable and resilient. Depending on the network power flows at any particular instant, the network may need to be re-configured to form new dynamic microgrids.

The simulation of power flow is carried out on the modified network of the 8500 node system, aggregating single-phase loads at three-phase nodes for different weather scenarios. Respective dynamic microgrid configurations for these weather scenarios are formed. 
Simulations are performed for three different fault cases with sunny weather: (1) grid failure (power loss at substation), (2) line outage between two interconnected microgrids and (3) fault within a microgrid. These fault cases demonstrate the significance of dynamic microgrid configuration.

Assumptions:

1. An aggregated balanced network is considered with 649 nodes and 648 lines.

2. The network is divided into 8 clusters.

3. Switches are present in each line so that the network can be reconfigured.

4. Solar PV systems are the only DER present in the system. Batteries are not considered.

\subsubsection{Base Case DMC Output (no fault considered)}

The cluster formation for dynamic microgrids in the sunny and cloudy scenarios are shown in Figure TCS-21 and Figure TCS-22 respectively. It can be observed that the microgrid with substation node (MG 1) has fewer nodes in the sunny weather case as compared to cloudy weather, as solar generation is sufficient in sunny weather to supply more loads.

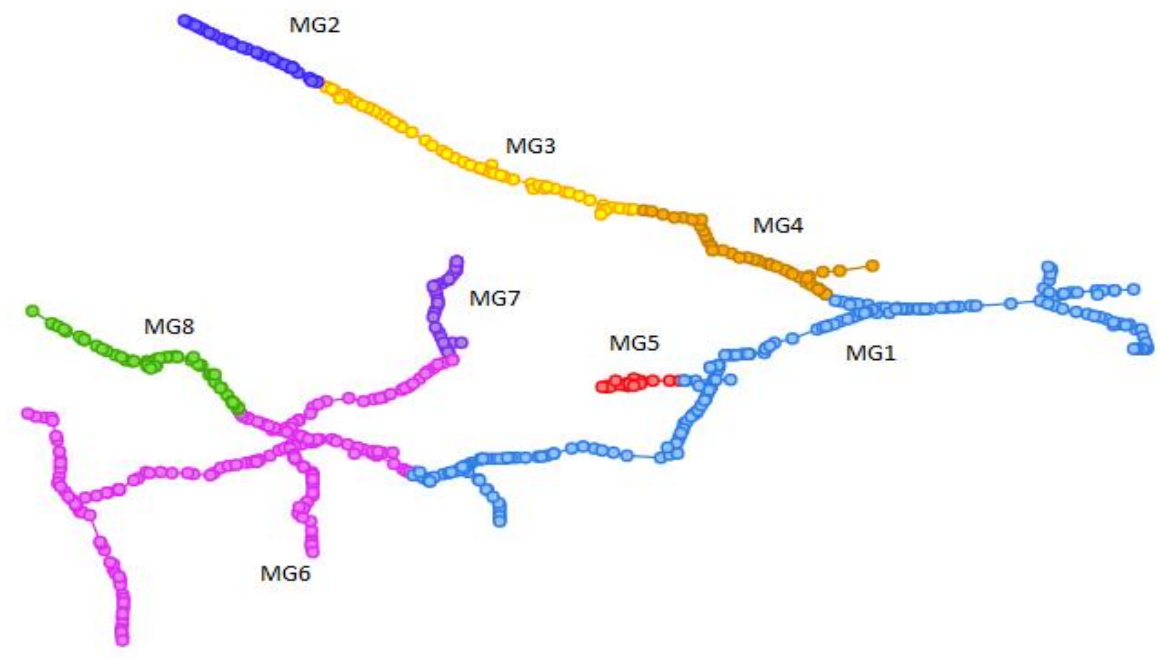

Figure TCS-21 Dynamic microgrid cluster formation in sunny scenario 


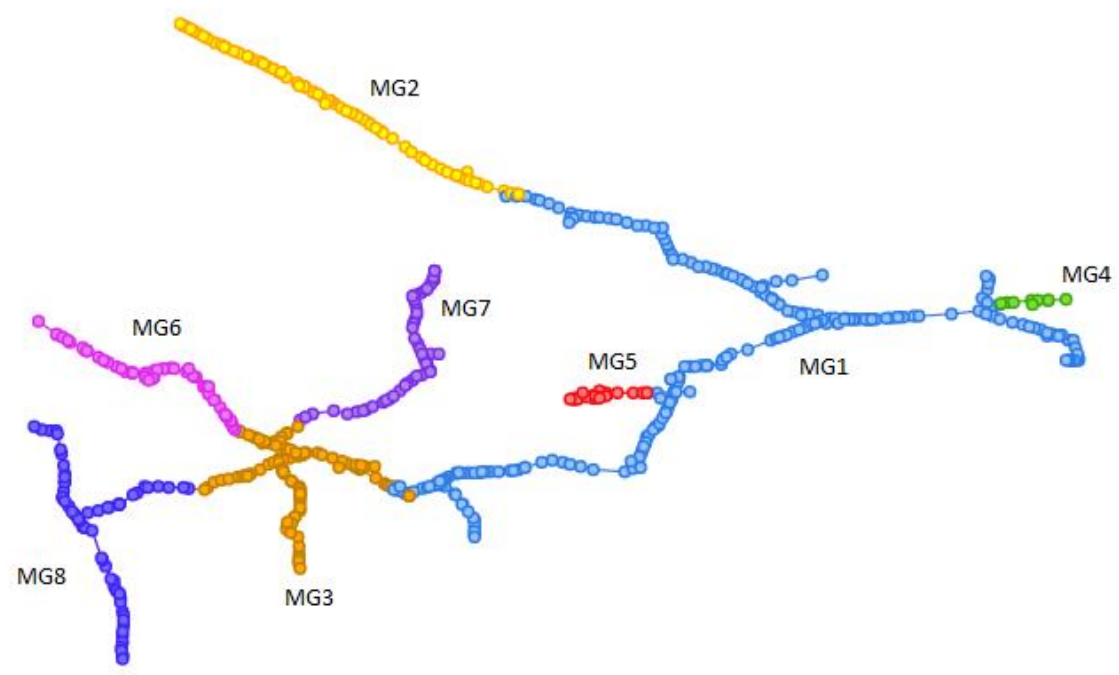

Figure TCS-22 Dynamic microgrid cluster formation in cloudy scenario

\subsubsection{Dynamic Microgrid Based Operations in Case of Faults}

\subsection{Case 1: Fault or Contingency Causing Outage of the Substation}

In Figure TCS-23, the grid supply fails at the substation and microgrid clusters are formed with solar generation as the major source (the fault occurs at the time of peak solar generation) in each cluster. All eight microgrids operate independently in isolation until the grid power is restored. Table TCS-2 describes the different operations with DMC and without ("Business-as-usual") for this case.

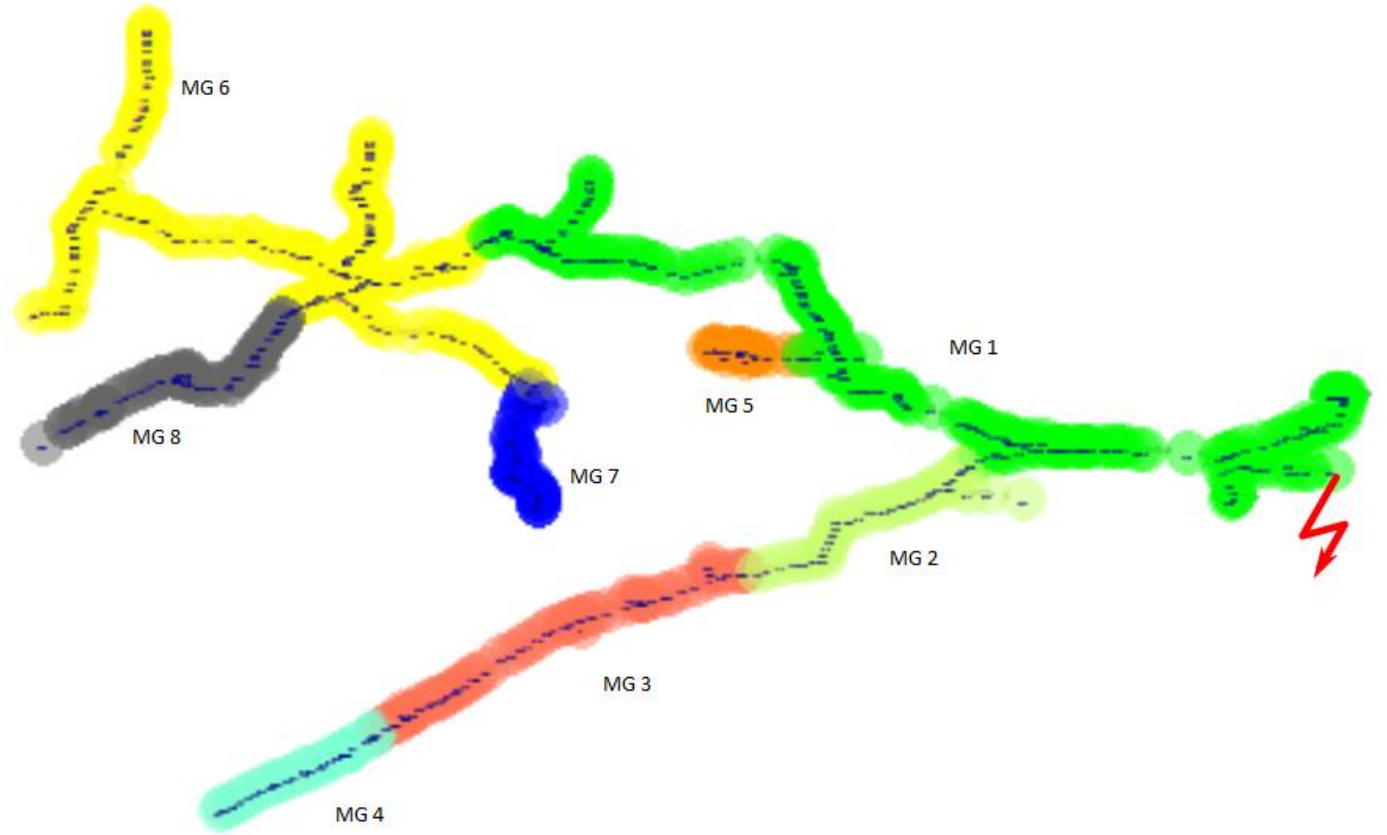

Figure TCS-23 Fault or contingency causing outage of the substation 
Table TCS-2 DMBO in Case 1

\begin{tabular}{|c|c|}
\hline Business-as-usual & DMC \\
\hline $\begin{array}{l}\text { In the event of a substation outage, } \\
\text { all the loads in the service area are } \\
\text { interrupted. The current grid } \\
\text { operation paradigm requires DERs } \\
\text { to disconnect from the grid until the } \\
\text { system returns to normal operation. } \\
\text { It is possible to restore the system if } \\
\text { a supply point is accessible from } \\
\text { another substation through tie- } \\
\text { switches. But in the } 8500 \text {-node } \\
\text { system, there is only a single } \\
\text { substation. Hence all loads are } \\
\text { affected. }\end{array}$ & $\begin{array}{l}\text { In DM-based operations, there are mechanisms in } \\
\text { place to allow the DERs to stay connected when the } \\
\text { grid is offline. This enables the loads to be served } \\
\text { based on the availability of generation within the } \\
\text { service area. As the DMs are identified to be nearly } \\
\text { supply-adequate, all the identified DMs transition to } \\
\text { islands when the substation is offline. In the } 8500- \\
\text { node system, we have identified } 8 \text { microgrids. Based } \\
\text { on the availability of internal load-generation } \\
\text { balance, partial load shedding might be required to } \\
\text { ensure stable operation of the microgrid. Even then, } \\
\text { the number of customers energized in this case is } \\
\text { higher than the business-as-usual scenario. }\end{array}$ \\
\hline
\end{tabular}

\subsection{Case 2: Fault on Line Between Two Microgrids}

In Figure TCS-24, a fault occurs between microgrids 2 and 3 (at boundary of dark blue and orange colors). Microgrid 3 is isolated from the grid and operates independently in islanded mode. Other microgrids are still connected to the grid.

Table TCS-3 describes the different operation with DMC and without ("Business-as-usual") for this case.

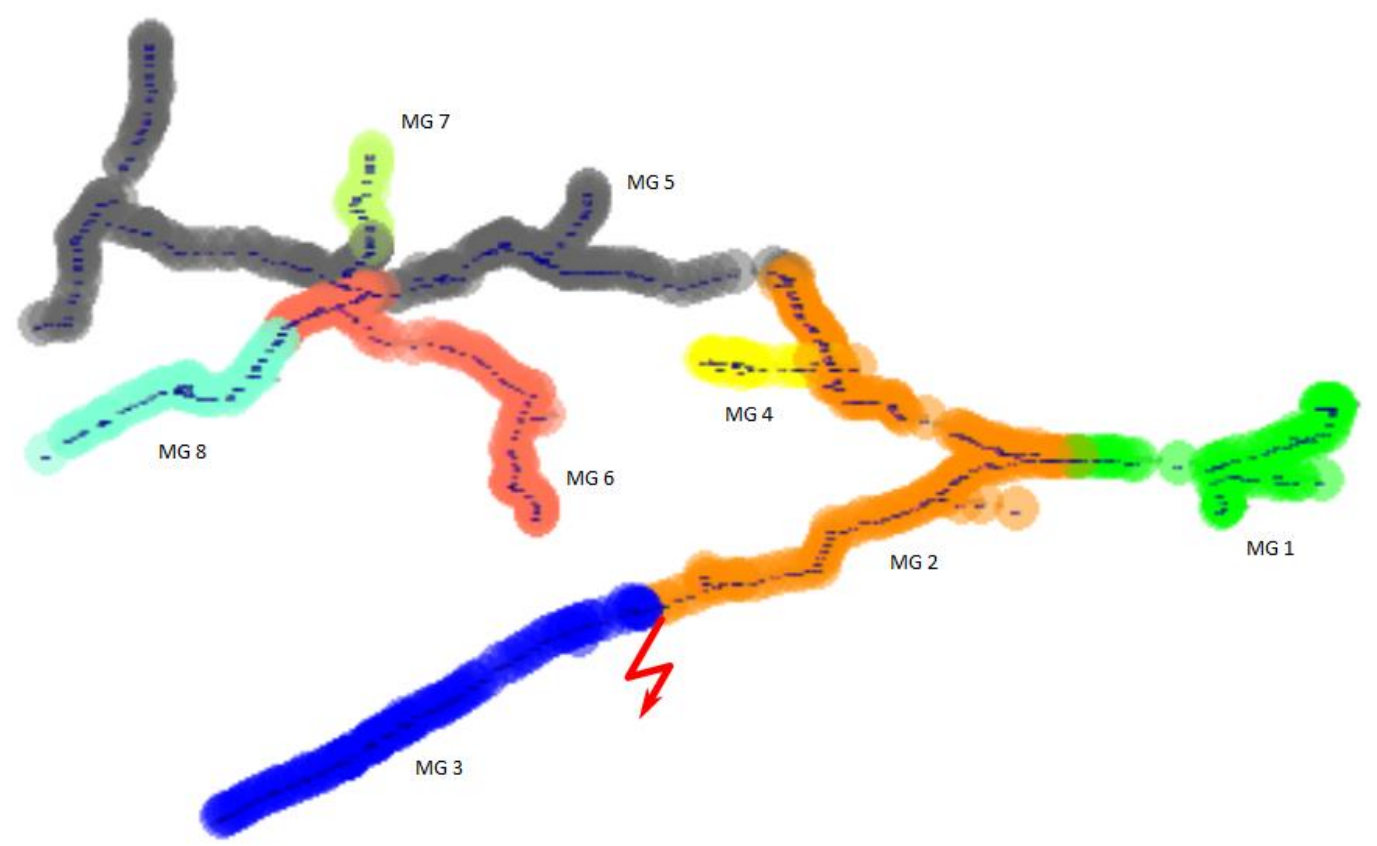

Figure TCS-24 Fault between two microgrids 
Table TCS-3 DMBO in Case 2

\begin{tabular}{|l|l|}
\hline Business-as-usual & DMC \\
\hline Any fault on a feeder is currently & In DMC, a fault between two microgrids is the \\
handled by disconnecting the entire \\
feeder until the service is restored by \\
elearing the fault or reconfiguring the \\
microgrids downstream from the fault are \\
immediately islanded as soon as the fault is \\
switches allowing reconfiguration, the \\
customers on the faulted feeder face \\
momentary interruptions due to the \\
the entire feeder, and the upstream customers \\
continue to be served. If the islanded microgrid(s) \\
iswitching operations required in \\
is supply-adequate to sustain islanded operation \\
until the fault is cleared, it will continue to operate \\
system has a single feeder, and any \\
fault in the system will cause \\
momentary interruption(s) for all \\
customers.
\end{tabular}

\subsection{Case 3: Fault Within a Microgrid}

In Figure TCS-25, a fault occurs within Microgrid 5 (Grey color). The majority of the nodes in this cluster are combined with cluster 1 (Green) so that maximum loads are served. The nodes downstream of the fault location will be disconnected from the system. This can be avoided using a tie-line which can connect this part with the nearest microgrid. Table TCS-4 describes the different operations with DMC and without ("Business-as-usual") for this case.

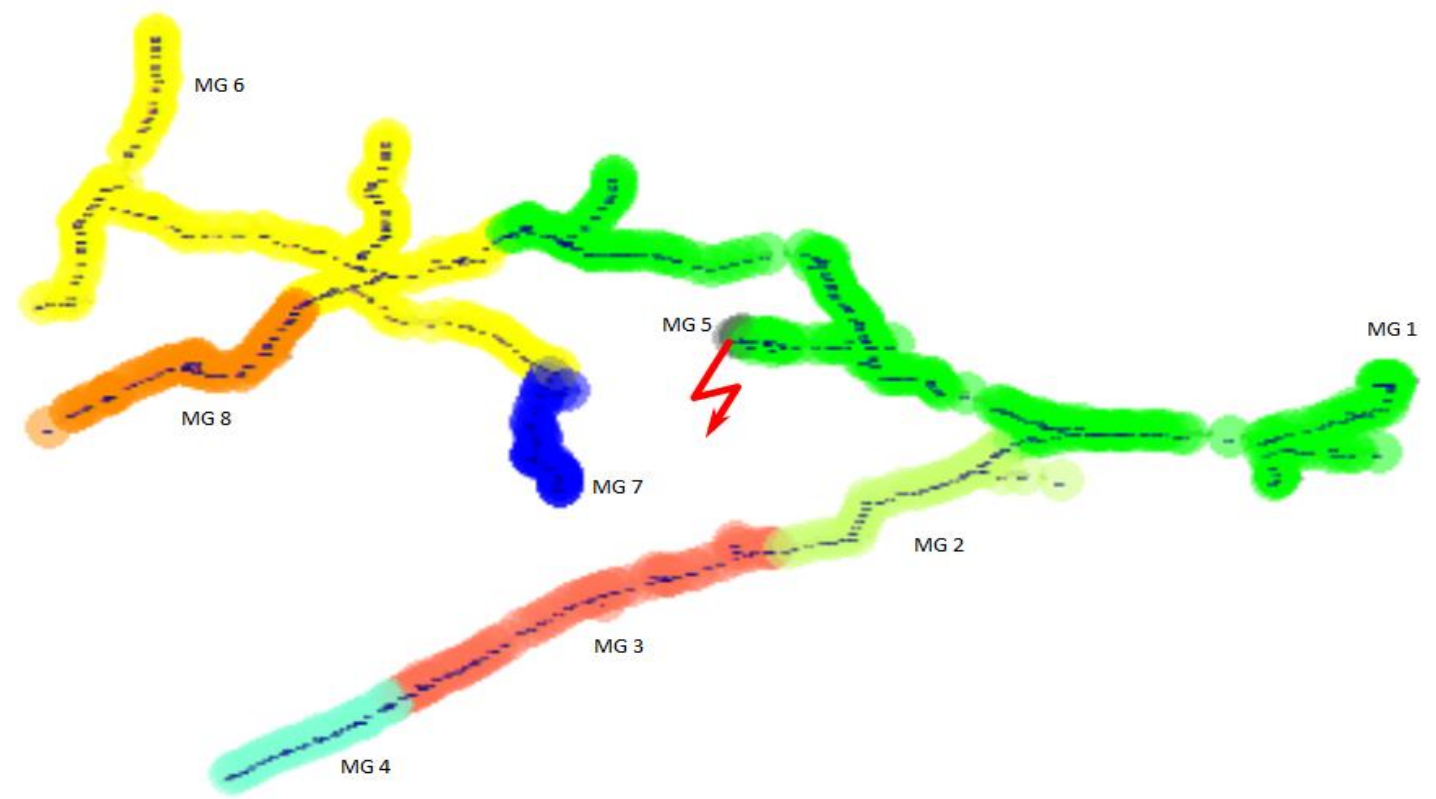

Figure TCS-25 Fault within a microgrid 
Table TCS-4 DMBO in Case 3

\begin{tabular}{|l|l|}
\hline Business-as-usual & DMC \\
\hline $\begin{array}{l}\text { The operation is similar } \\
\text { to the case when there is } \\
\text { a fault between }\end{array}$ & $\begin{array}{l}\text { In contrast to the case where the fault is between microgrids, } \\
\text { an internal fault will necessitate reconfiguring the microgrid } \\
\text { microgrids. }\end{array}$ \\
$\begin{array}{l}\text { within which the fault occurs. Islanding the faulted microgrid } \\
\text { will allow unaffected parts of the network to stay connected } \\
\text { with a neighboring microgrid. The customers within the faulted } \\
\text { microgrid will face multiple interruptions as the service is } \\
\text { restored by isolating the faulted line, and joining the unaffected } \\
\text { nodes to other microgrids through switching operations. }\end{array}$ \\
\hline
\end{tabular}

The results of DMC on the 8500 network are presented in Table TCS-5 for all three cases considered.

Table TCS-5 Comparison of Load Interruptions without and with DMC

\begin{tabular}{|c|c|c|}
\hline \multirow{2}{*}{ Case } & \multicolumn{2}{|c|}{ Amount of load interrupted } \\
\cline { 2 - 3 } & Business-as-usual & DMC \\
\hline 1: Substation outage & $15,694 \mathrm{~kW}$ & $\begin{array}{c}7944 \mathrm{~kW} \text { (allowing DERs to } \\
\text { energize the microgrids) }\end{array}$ \\
\hline $\begin{array}{c}\text { 2: Fault on line 509-645 } \\
\text { (between MG2 and MG3) }\end{array}$ & $\begin{array}{c}15,694 \mathrm{~kW} \text { (momentary*) } \\
\text { 3: }\end{array}$ & (MG3 is supply adequate) \\
\hline $\begin{array}{c}\text { 3ault on line 32-35 } \\
\text { (within MG5) }\end{array}$ & $\begin{array}{c}15,694 \mathrm{~kW} \text { (momentary*) } \\
26.43 \mathrm{~kW} \text { (sustained) }\end{array}$ & $\begin{array}{c}26.43 \mathrm{~kW} \text { (MG5 islanded. } \\
\text { Interruption until fault is } \\
\text { isolated) }\end{array}$ \\
\hline
\end{tabular}

* Momentary interruption until switching is done to isolate faults, assuming there are no tieswitches present in the entire network.

Power flow on the 8500 system is simulated and an instance is obtained in which the total load of the system is $15,694 \mathrm{~kW}$, total solar generation is $7,750 \mathrm{~kW}$, and substation supplied power is $7,944 \mathrm{~kW}$. In the case of the substation outage, the entire $15,694 \mathrm{~kW}$ load is interrupted when there is no dynamic microgrid. With DMC, the grid is disconnected, and available solar energy is supplied to the load and the remaining $7944 \mathrm{~kW}$ load is disconnected. In Case 2 Business-asusual, the fault between MG2 and MG3 interrupts the complete load of $34.65 \mathrm{~kW}$ in MG3. In presence of DMC, the isolated MG3 operates in islanded mode with all its solar generation supplying the load. Similarly, in Case 3 Business-as-usual, the fault within MG5 interrupts the entire $26.43 \mathrm{~kW}$ load. With DMC, the entire load of $26.43 \mathrm{~kW}$ within the microgrid is interrupted if not connected to the nearest microgrid using tie-lines.

As shown in Table TCS-5, in the case of islanded microgrids, all power is sourced from PV systems. In Case 1, green energy utilization is $100 \%$ due to curtailment of loads (reduction of $7,944 \mathrm{~kW}$ that had been sourced from the bulk grid). In Case 2 and 3, all load in islanded microgrids is served from PV systems. Considering line losses, they are reduced in Case 1 due to reduction of line loading with curtailment of load. 


\subsubsection{TE Market Simulation}

The market model is simulated for the truncated grid in the absence of microgrids, i.e. considering the entire network as grid-connected for sunny and cloudy weather scenarios. The market model takes bid data and calculates market clearing price and sends this as a TE signal to all market participants. The bulk grid power (supply by retailer) is considered as a retail market participant along with individual homes (bids to sell solar PV). The bid prices of each supply source are bid into the market clearing algorithm. The TE controller compares the resulting clearing price with its submitted bid price and changes the state of the device accordingly.

Assumptions:

1. Fixed loads considered are ZIP loads ${ }^{2}$ with schedules defined for every time block.

2. Price responsive loads are thermostat loads (heating and cooling mode).

3. The thermostat settings (e.g., thermostat dead band, heating and cooling setpoints) are the same for all houses.

4. The magnitude of prices bid for customer solar generation and substation generation are in a similar range.

The plot in Figure TCS-26 shows the market clearing for the entire day. It can be seen that the quantity cleared decreases when there is a significant increase in the Market Clearing Price (MCP). This decrease is especially evident in the evening hours.

In the period between 7:00 to 8:00 and 10:00 to 11:00, the MCP is zero. This is because during this period none of the HVAC loads bid into the market, which results in no market clearing. The quantity cleared during this period is only the essential load and does not include any HVAC load. The bid prices for solar and substation generation are set in the same range such that the MCP is high in some cases when solar is available.

Figure TCS-27 shows that the HVAC runs when the bid price is greater than the MCP for a given time interval. When the MCP is greater than the bid price, the HVAC shuts off for that interval.

${ }^{2}$ ZIP are the coefficients of a load model comprised of constant impedance, constant current, and constant power loads. A ZIP coefficient load model is used to represent power consumed by a load as a function of voltage. 


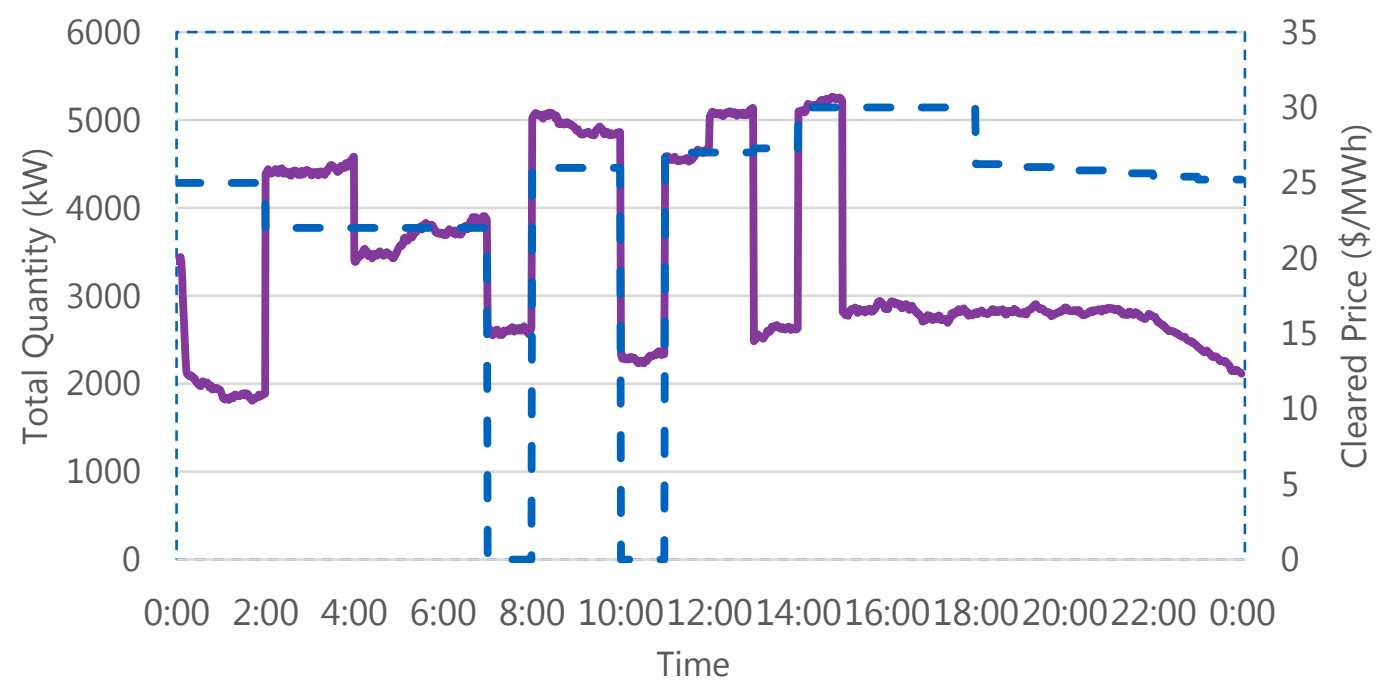

—Total Quantity - - Cleared Price

Figure TCS-26 Market clearing

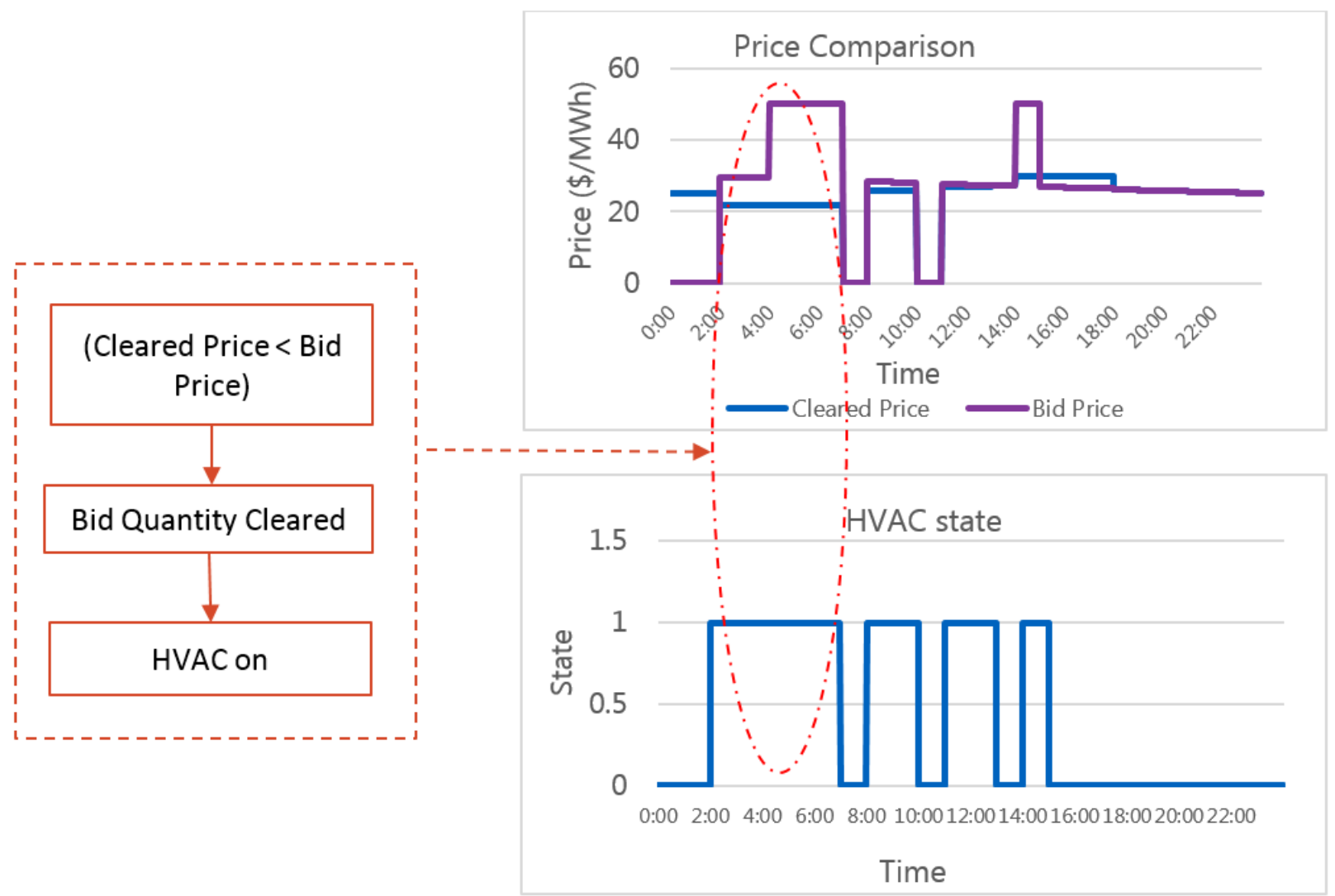

Figure TCS-27 State of HVAC 
Every controller bids in the market in the form of a price-quantity pair. For every controller, we consider the load to be categorized in two types: fixed load (which needs to be ON irrespective of the price) and variable load (which is flexible and responsive to the price). HVAC is considered as a variable type. The quantity bid is formed by the summation of these two load types. After market clearing, if the price bid by the controller is higher than the clearing price, its corresponding quantity gets cleared, which means it can switch on its variable plus fixed load, otherwise only the fixed load will be supplied. As seen from Fig. TCS-27, from 2:00 to 7:00, the cleared price is less than the bid price, which means the entire quantity bid by that controller was cleared which permits the HVAC load to run.

Figure TCS-28 shows the substation power, total house load and total HVAC load of all houses for the cloudy scenario. As the HVAC loads are controlled, there are instances in which HVAC loads are completely off, thus resulting in the pattern shown. In the case of cloudy weather, the substation power is greater from 14:30 to 16:30 when cloud cover is present. Substation power is less and flat during sunny weather during this period as shown in Figure TCS-31, as most of the loads are supplied by solar. Figure TCS-30 and Figure TCS-33 shows the minimum, average and maximum temperature of all houses. It can be seen that the average temperature of all houses lies within $23{ }^{\circ} \mathrm{C}$ to $27^{\circ} \mathrm{C}\left(73^{\circ} \mathrm{F}\right.$ and $\left.81^{\circ} \mathrm{F}\right)$. Figure TCS-28 to Figure TCS-33 are plotted for every five minutes in a day when the market is cleared.

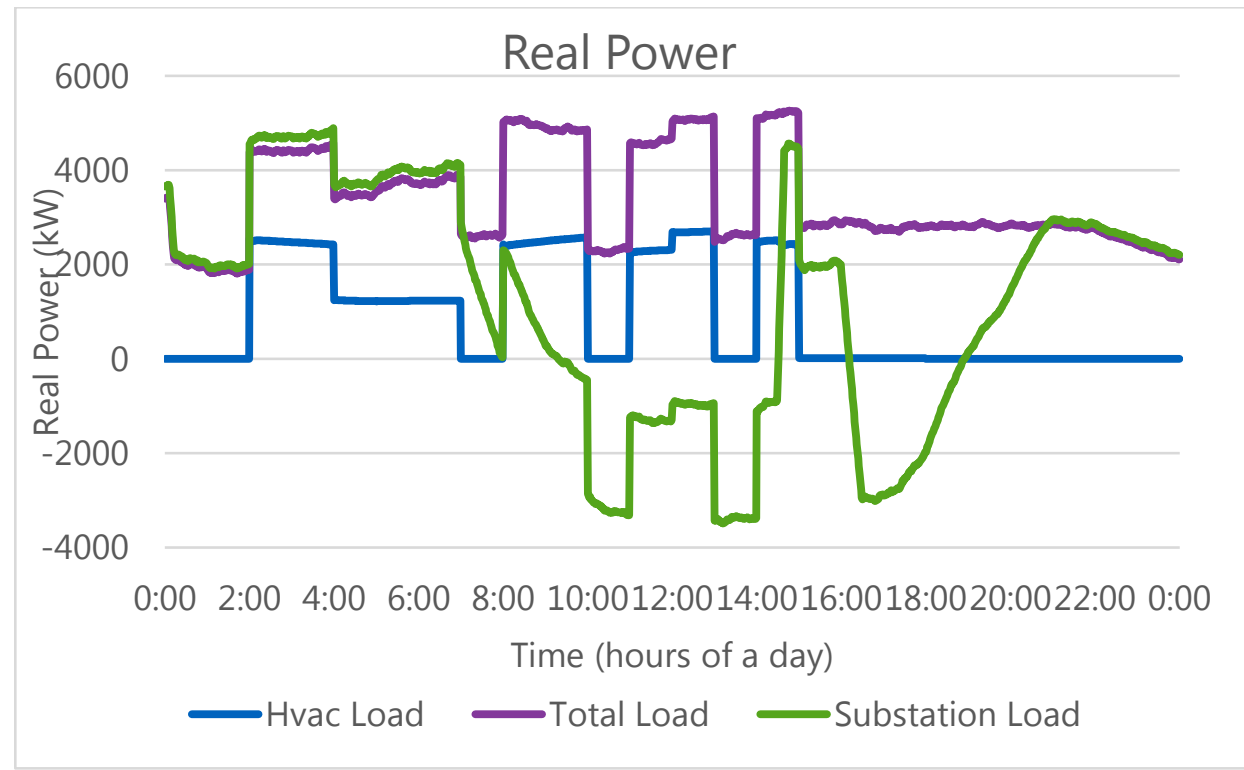

Figure TCS-28 Real power (Substation, House load and HVAC) on a cloudy day 


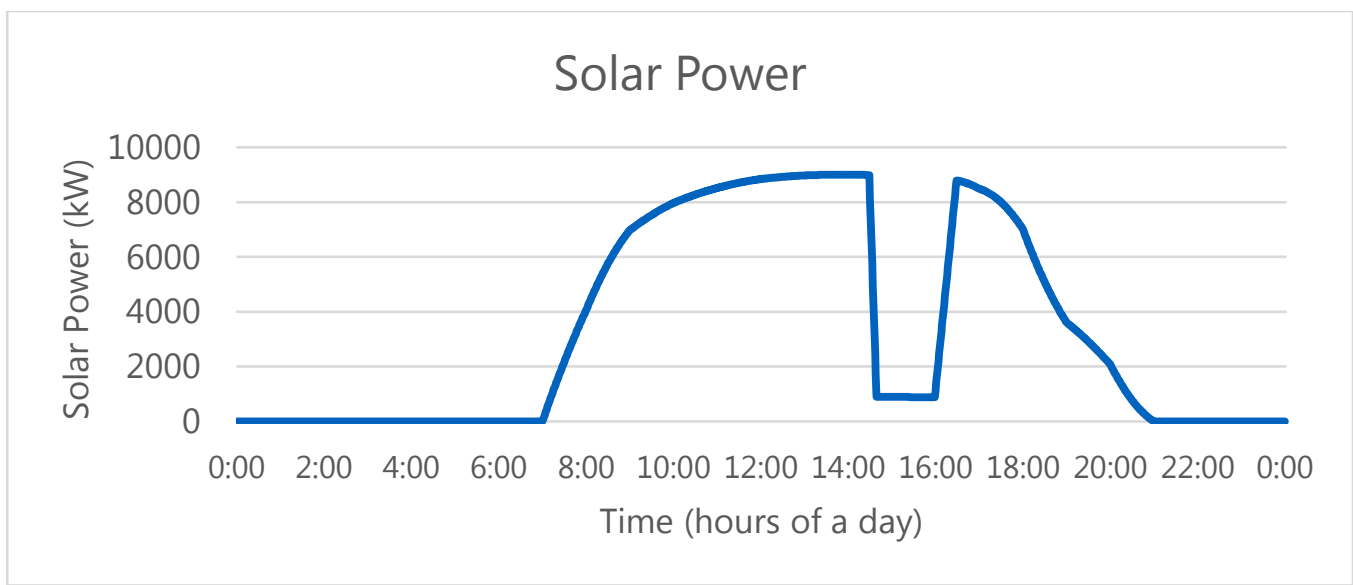

Figure TCS-29 Solar PV power on a cloudy day

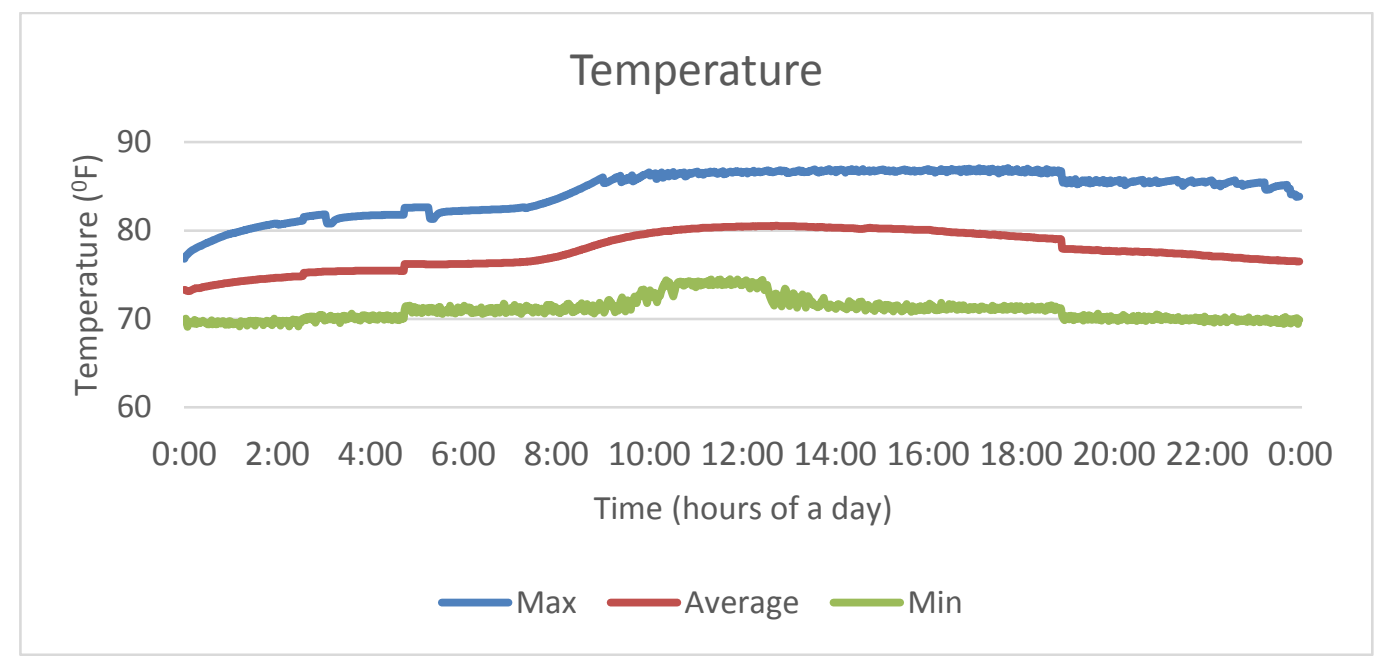

Figure TCS-30 Max, Min and Average temperature of houses on a cloudy day

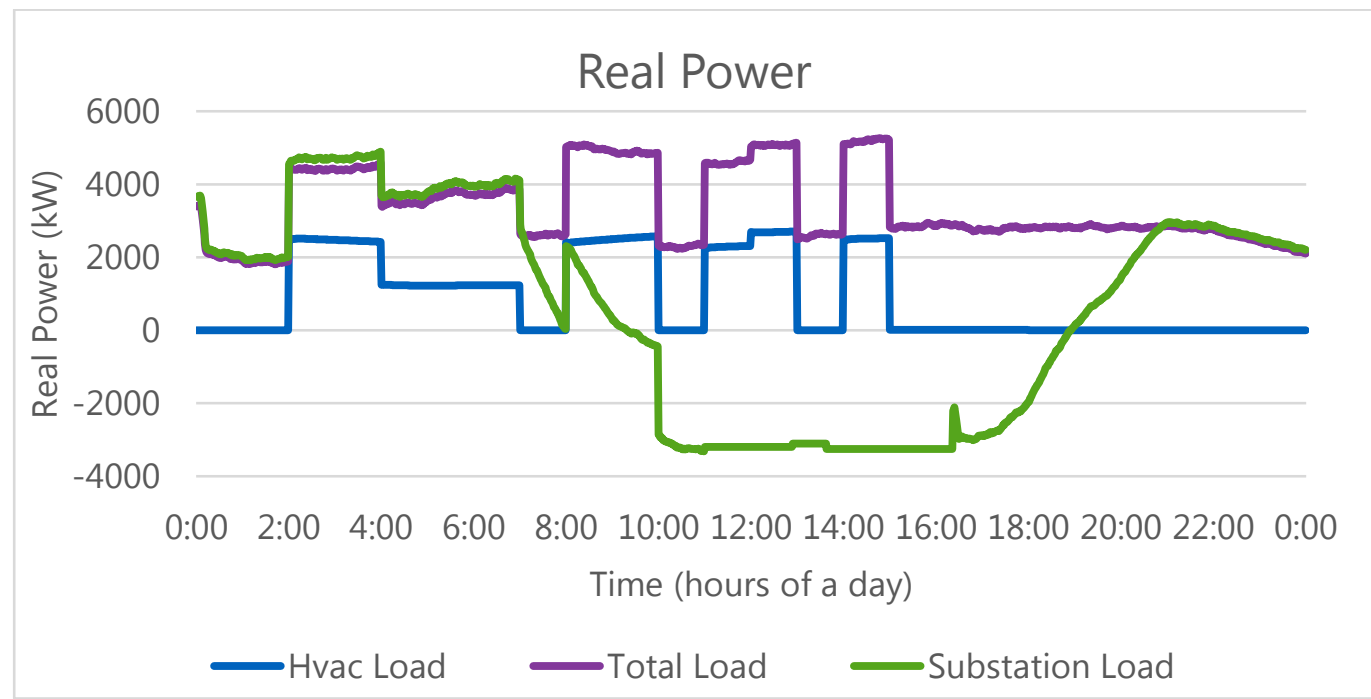

Figure TCS-31 Real power (substation, house load and HVAC) on a sunny day 


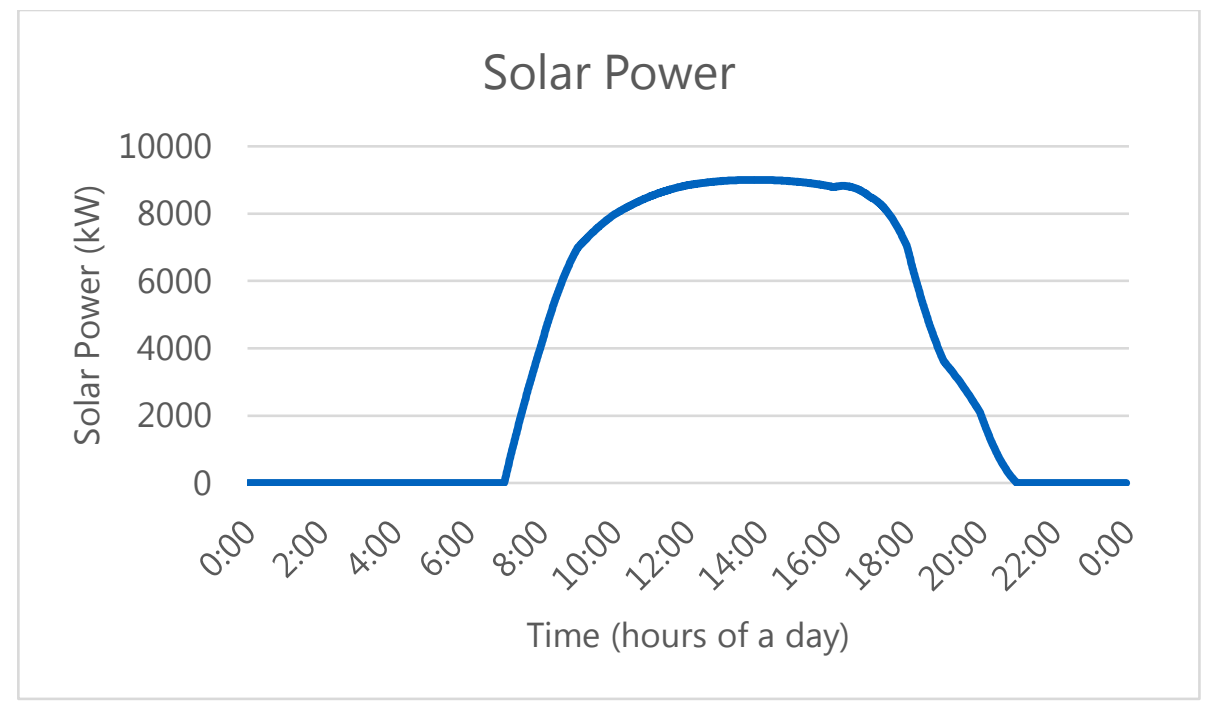

Figure TCS-32 Solar PV power on a sunny day

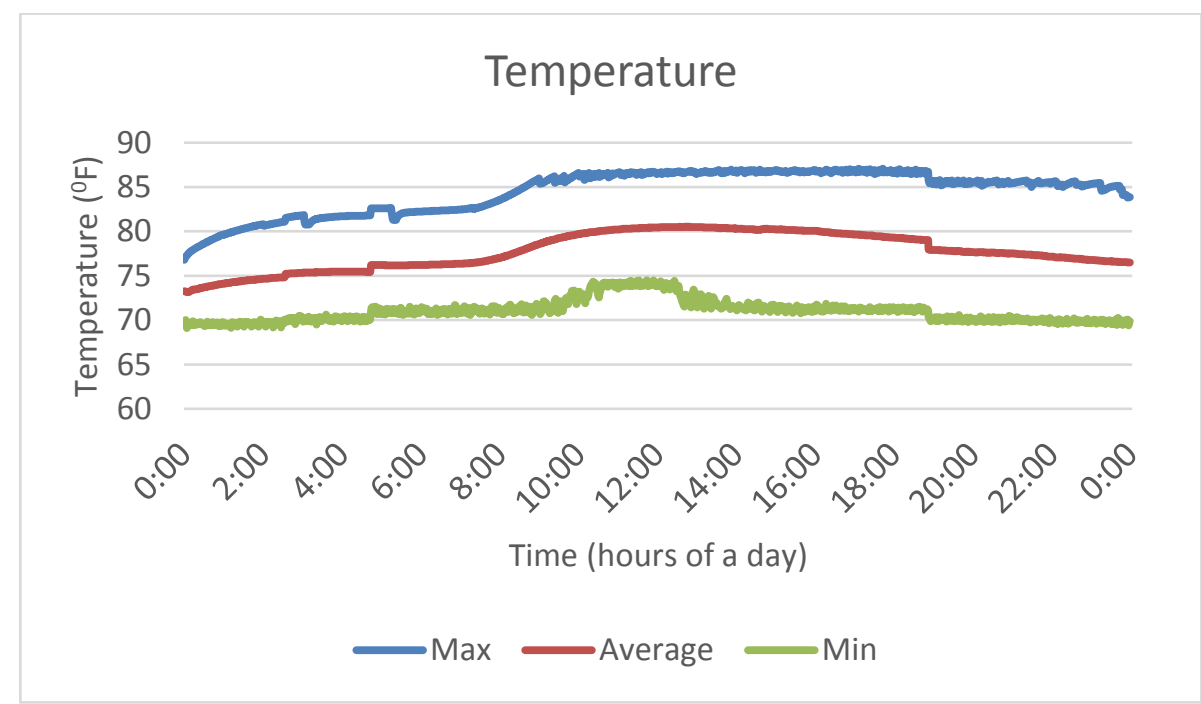

Figure TCS-33 Max, Min and Average temperatures of houses on a cloudy day

For the sunny weather scenario, there is continuous solar irradiance in the afternoon. Due to this, it can be seen that the power supplied by the substation decreases during the same period.

For the cloudy weather scenario, due to cloud cover from 14:30 to 16:30, there is low solar power and thus a sudden increase in the substation power during the same period.

\subsection{References}

[1] Joel Jose, Anupama Kowli, Vaibhav Bhalekar, Krishna V Prasad, Narayanan Rajagopal, "Dynamic microgrid-based operations: A new operational paradigm for distribution networks", ISGT Asia 2016

[2] GridLAB-D, https://www.gridlabd.org/. 


\title{
Section 5 MIT Team Report: Enabling operationally feasible prosumer-centric TEM
}

\author{
Marija Ilic, Rupamathi Jaddivada \\ Massachusetts Institute of Technology, Boston, MA
}

\begin{abstract}
Transactive Energy Management (TEM) is modeled and simulated using our general Dynamic Monitoring and Decision Systems (DyMonDS) framework and its supporting computer platform named Scalable Electric Power System Simulator (SEPSS) [1]. This home-grown simulation platform co-simulates interactions of distributed energy resource (DER) models with the SEPSS compute node. The compute node coordinates market clearing by co-simulating interactions with the third-party AC optimal power flow (AC OPF) to perform system level optimization. DERs send their bids, and the compute node computes and sends locational marginal prices (LMPs) and optimal schedules back to the DERs. This process was simulated using IEEE 8500 bus distribution system on the MIT supercomputer. It was shown for the first time how to implement TEM by dynamically exchanging LMPs while, at the same time, controlling voltages to meet ANSI C84.1 standard. Using AC OPF is the key to optimizing voltages, further ensuring that real and reactive power are balanced, subject to voltage and grid constraints.

All TEM cases are simulated using the IEEE 8500 bus system. More generally, this experiment has shown that significant integration of DERs, including solar PVs, critically hinges on how operationally feasible their integration is. Except for the highly isolated stand-alone households equipped with very small storage targeted to serving their own plug-load, the distribution grids and micro grids will be essential for delivering power provided by the DERs to the plug-load locations. However, at present the role of local utilities is not well-defined and the incentives for supporting large integration of DERs may not be explicit. We have attempted to illustrate the essential role of grid management in deploying efficient and clean power in scalable ways.

The simulation analyses and economic and technical metrics performances provided in this paper can be used as the basis for further discussion of some key open questions and for proposing possible ways forward. Moving forward requires considerable depth in assessing the issues and proposing protocols and standards that may create an environment in which different stakeholders are paid and pay according to the right incentives and tariffs. The resulting metrics support the expected conclusions that both technical and economic performance depend on the technical functions performed by different stakeholders, as well as on the valuation of these functions. For example, maintaining voltages within the operating limits is ultimately required so that the electricity service to the small end users meets ANSI C84.1 standard. These limits can be observed and maintained in many non-unique ways, such as by either solar PVs participating in grid-level voltage regulation, or by the distribution grid regulating its controllable hardware, such as transformer and shunt capacitor/transformer equipment. It is shown in this paper that ultimately the voltage support can be provided in a very effective way by the regulation of substation voltage itself.
\end{abstract}


An important technical detail is that, depending on the functionalities/ sub-objectives of different stakeholders, the voltage profile can be either: (1) simply analyzed by running power flow to assess whether the voltages will be kept within the limits and, if not, local PVs would have feedback control to respond to deviations away from the acceptable ranges; or, (2) more traditionally, the grid operator sub-objective could be to minimize delivery losses, without considering the need to differentiate between utility and DER power delivery. In this paper a third alternative is considered in which voltage is modeled as a decision variable, along with the real power, to support maximizing social welfare, for example. This is done by using an extended AC OPF capable of optimizing both voltage and real power dispatched/consumed. As the basic observation, one can see from the simulations presented that when net load increases, the power flow analysis shows that the resulting voltages decrease. However, when AC OPF is used to support delivery of increased system load, the voltages generally increase as a result of adjusting controllable voltage set points elsewhere, for example, set points of controllable grid equipment or the voltage set points of controllable solar PVs. This optimization then makes room for delivering more net system load without violating ANSI standards required by the end users. Significant benefits from such voltage optimization are reported.

Establishing incentives to the right stakeholders for supporting power delivery and voltage according to ANSI C84.1 standard is quite complex. These incentives can be rolled into typical two-part tariffs comprising fixed charges and pass-through variable cost, such as fuel cost, in particular. The problem with fixed charges at guaranteed rate-of-return is that they are strictly volumetric and do not differentiate between controllable or non-controllable equipment. This volumetric (capacity-based) approach to tariffs is one of the key causes of utilities' concerns with DERs creating competition to their service. It is shown in this paper that the issues regarding energy bills become convoluted in the TEM environment depending on which pricing methods are used to give incentives to the DER market participants. The three options considered are fixed two-part tariffs; time of use (TOU) tariffs (again based on a two-part approach or averaged variable cost approach); or entirely LMP-based. We provide ample evidence of how this choice affects all economic metrics. 


\section{Table of Contents}

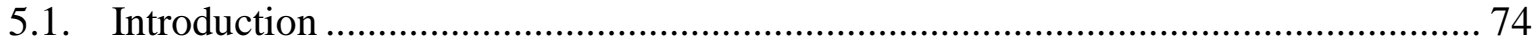

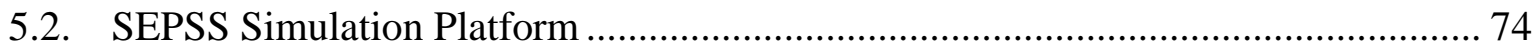

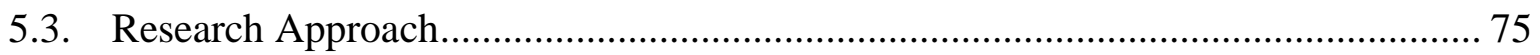

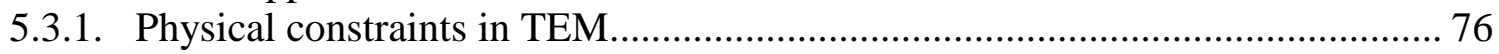

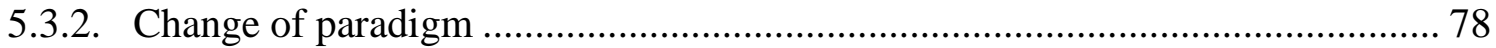

5.3.3. Distributed decision making for TEM............................................................ 79

5.3.4. Non-unique performance objectives: the key role of voltage optimization and

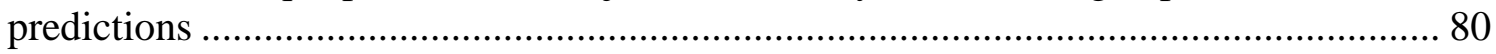

5.3.5. Optimization subject to hard or soft constraints............................................... 81

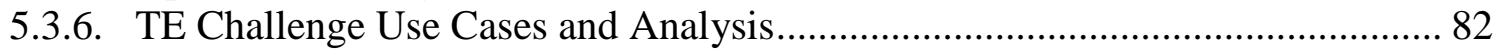

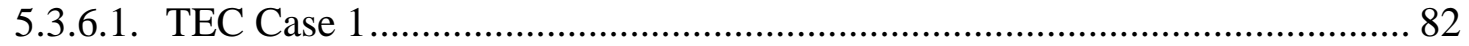

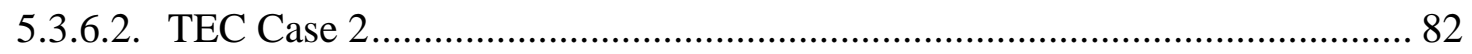

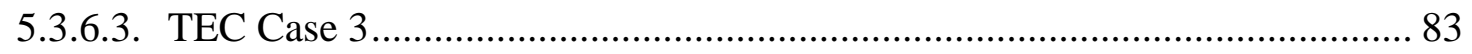

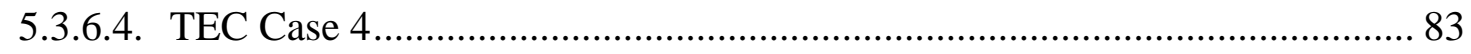

5.3.7. Roles and performance metrics of different TEM participants ........................... 84

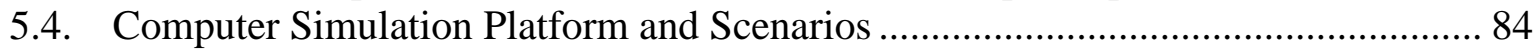

5.4.1. SEPSS Computer Simulation Platform for TEM …………….......................... 84

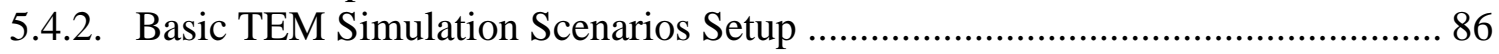

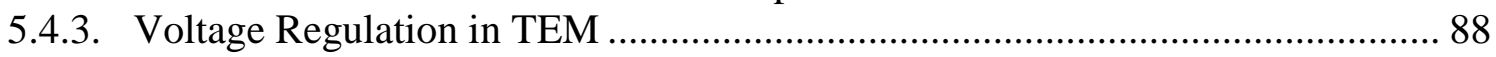

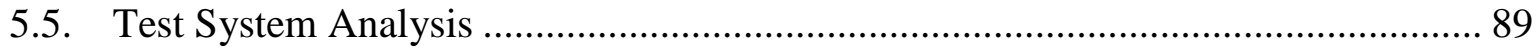

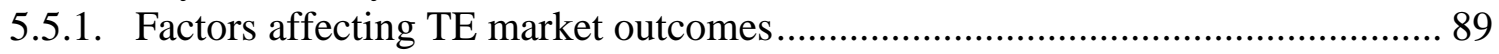

5.5.1.1. Effect of comfort constraints on the elasticity of bids .................................. 90

5.5.1.2. Comparison of economic metrics ............................................................. 93

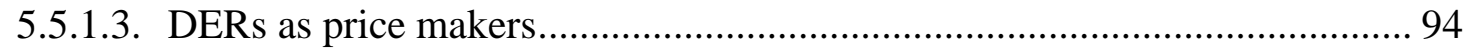

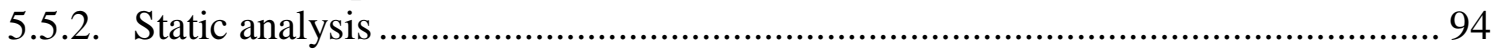

5.5.2.1. Observations Based on Numerical Findings .................................................. 99

5.5.3. Continuous Time 24-hour MPC-based TEM Simulations ................................. 100

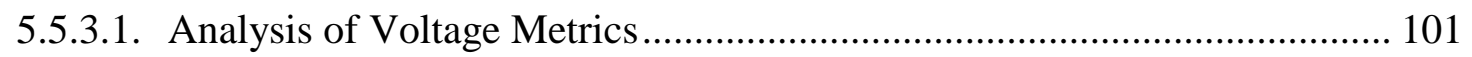

5.5.3.2. Power Generation/Consumption Metrics ..................................................... 103

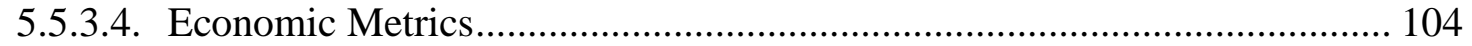

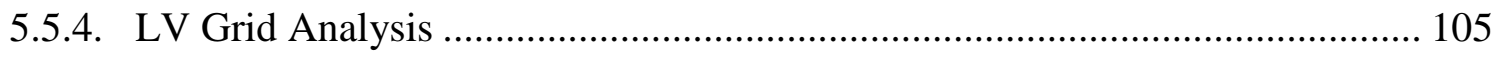

5.6. Future Utility Role in Transactive Energy Markets ................................................ 107

5.7. Preliminary Conclusions: Performance-based Grid Regulation based on TEM Peak Load

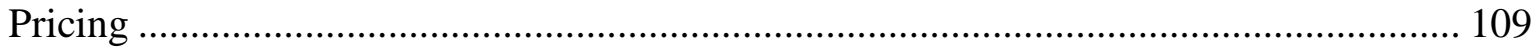

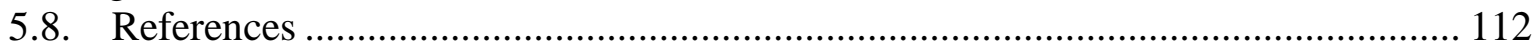




\section{List of Acronyms}

$\begin{array}{ll}\text { 3Rs } & \text { Rules, rights and responsibilities } \\ \text { ACM } & \text { Abstract Component Model } \\ \text { ACOPF } & \text { AC Optimal Power Flow } \\ \text { BPS } & \text { Bulk Power System } \\ \text { CMU } & \text { Carnegie Mellon University } \\ \text { EESG } & \text { Electric Energy Systems Group } \\ \text { DER } & \text { Distributed Energy Resources } \\ \text { DPS } & \text { Distribution Power Systems } \\ \text { DyMonDS } & \text { Dynamic Monitoring and Decision Systems } \\ \text { HLA } & \text { High Level Architecture } \\ \text { HVAC } & \text { Heating, Ventilating and Air Conditioning } \\ \text { ISO } & \text { Independent System Operator } \\ \text { LMP } & \text { Locational Marginal Price } \\ \text { LV } & \text { Low Voltage } \\ \text { MPC } & \text { Model Predictive Control } \\ \text { MV } & \text { Medium Voltage } \\ \text { NW } & \text { NETSSWorks } \\ \text { PoC } & \text { Point of Connection } \\ \text { QoS } & \text { Quality of Service } \\ \text { PLP } & \text { Peak Load Pricing } \\ \text { p.u. } & \text { Per unit } \\ \text { PV } & \text { Photovoltaic } \\ \text { TE } & \text { Transactive Energy } \\ \text { TEC } & \text { Transactive Energy Challenge } \\ \text { TEM } & \text { Transactive Energy Management } \\ \text { TOU } & \text { Time-Of-Use } \\ \text { SCADA } & \text { Supervisory Control and Data Acquisition } \\ \text { SEPSS } & \text { Scalable Electric Power System Simulator } \\ \text { SGRS } & \text { Smart Grid in a Room Simulator } \\ \text { WH } & \text { Water Heater } \\ & \end{array}$




\subsection{Introduction}

Research was performed in the Laboratory for Information and Decision Systems which is one of the laboratories in the newly formed Institute for Data, Systems and Society at MIT. The DyMonDS framework was conceived at the Carnegie Mellon University (CMU) Electric Energy Systems Group (EESG) under the leadership of Marija Ilic. In 2014 NIST and EESG began a collaborative project on Smart Grid in a Room Simulator (SGRS) which is currently being generalized and expanded into a Scalable Electric Power System Simulator (SEPSS) [1, 2, 3]. Our participation in the TE Challenge is in part supported by the NIST project at MIT [4].

The initial motivation for SGRS was to design a sufficiently general computer platform that can emulate the effects of cyber (control, communication) and electricity markets on physical performance of rapidly emerging complex electric power systems. The main strength of this platform is that it is fundamentally based on aligning information processing with physical phenomena and economic decisions. We have only recently established a full theoretical foundation for this design [5,6]; our early intuition, based on understanding the structure of hierarchical control in complex electric power grids, has been fully confirmed.

The intent was not to make real-time simulators, but, instead, a poor-women real time digital simulator in which the interdependencies between technical and economic sub-objectives across spatial, temporal and organizational hierarchies are shown using simulations, and causes and effects are captured in causal ways. Limiting ourselves to simulations has been intentional, as we believe that it is essential to assess different causes and effects in the rapidly changing industry in the context of candidate architectures prior to engaging in hardware developments. Results of these early efforts can be found in the CMU EESG reports to NIST $[2,3]$ and in $[7,8]$. At the early TE Challenge meetings, DyMonDS was recognized as one of the two frameworks for advanced control design in the changing electric power industry, together with GridLab-D. At CMU, SGRS was going to become scalable by using SGRS shell, which communicated across all idle computing resources in the ECE Department. Once transplanted to MIT, the scalability was attempted by using Supercloud [9], instead of many distributed computers. This is currently work in progress as part of the SEPSS design in collaboration with the MIT-Lincoln Labs Supercloud team [10]. Scalability is being attempted by exploiting a combination of technical and economic sub-objectives with the physical grid structure, fundamentally based on primaldual optimization.

\subsection{SEPSS Simulation Platform}

The SEPSS simulation platform is based on the conceptual notion of DyMonDS which proposes that any power system agent can be abstracted to a level of detail that only its interface variables are exposed to its neighbors. This not only ensures privacy but also enhances integration of components to assess a large system and its interconnections. Based on this idea, the SGRS shell makes the first attempt to align the physics-based structure with the partitioning of computing jobs to several processes. The shell facilitates allocation of computing resources, sets up the TCP/IP communication channels between different modules and initiates the simulation. The simulation platform is High Level Architecture (HLA)-compatible [11], allowing integration with third-party software. As a part of this phase of TE Challenge, we have implemented dynamic inter-operability between two simulation platforms: an advanced grid analysis software 
called NETSSWorks and our software platform. The sketch of the components that constitute the simulation platform and its links to external modules is shown in Figure MIT-1.

This figure shows interactions that may happen within the system. Here, the module named 'House' is one of the transactive agents, but industrial customers or any other component trading in an energy market can be modeled and treated as such green colored boxes. Several of these models together constitute a community, and they are either coordinated by a community coordinator or through distributed peer-to-peer decisions. Our simulation platform does not recommend specific architectures for design and in fact is designed to be general enough for testing any given architecture in a rapid manner. Each module in the platform can be plugged in or out without having to change any of the simulation components. These concepts are similar to those proposed in the NIST ACM [12], which characterizes resources and their interfaces.

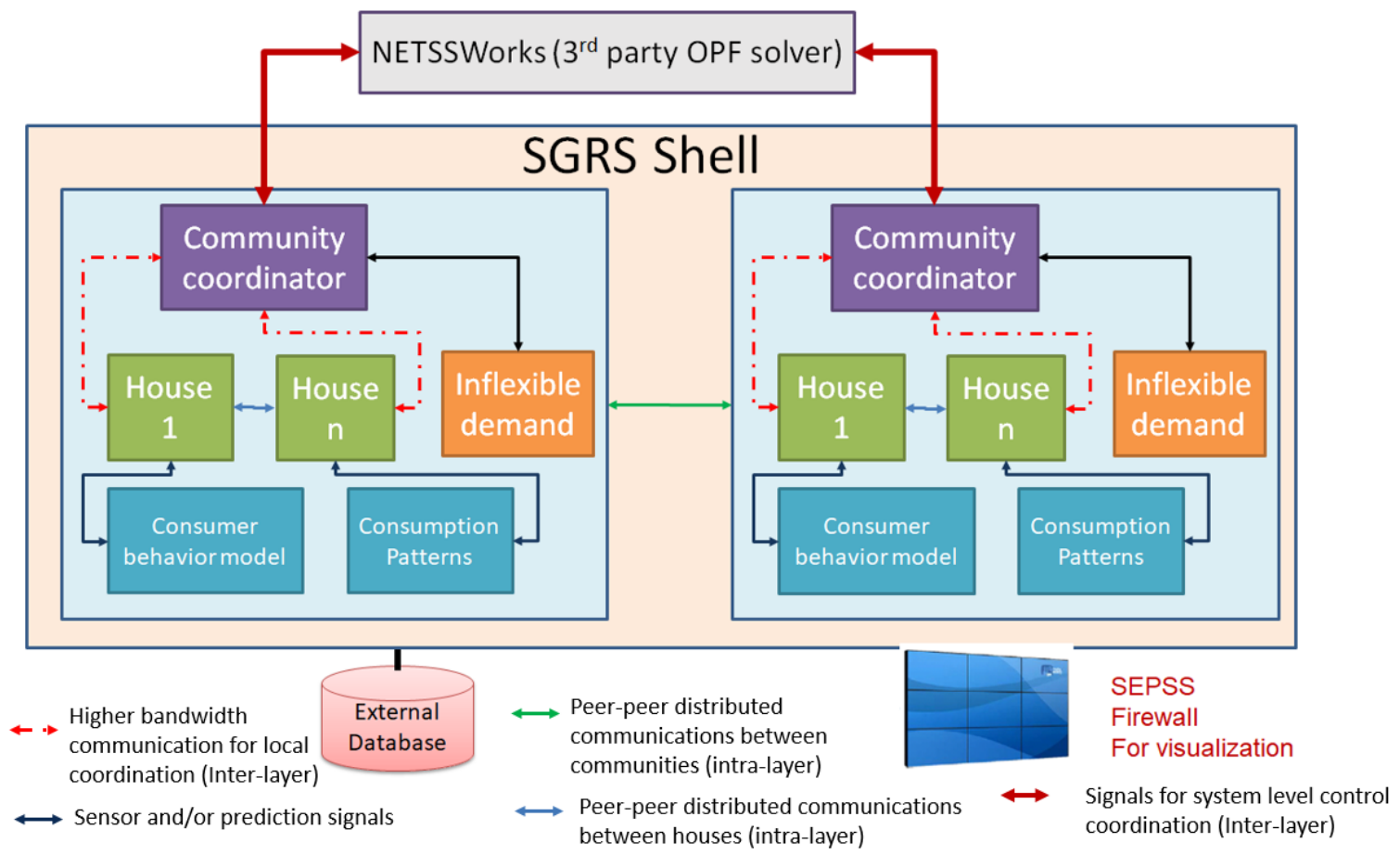

Figure MIT-1 Interaction of software modules within SEPSS computer platform [13]

The simulation platform is still under development, with focus on making the simulations scalable to very large systems, by supporting computer clustering, aggregation and decomposition according to the physical and organizational structure of an electric power system that is rich in both temporal and spatial scales.

\subsection{Research Approach}

In this report, we consider the IEEE 8500 distribution grid used by the entire team. Overlaid on this physical grid is Supervisory Control and Data Acquisition (SCADA), enabling its on-line operation and supporting electricity markets. As shown in Figure MIT-2 [14], today's on-line data-enabled decision-making and commands are limited to the Bulk Power Systems (BPS) only (orange solid lines in Figure MIT-2). The aggregate loads are monitored at the substation levels, 
as point of connection (PoC) between the BPS and its distribution power systems (DPS). The operation of a DPS is typically pre-programmed, based on historic loads. These loads have not participated in demand response, except at best in Time-of-Use (TOU) programs. Transactive energy management (TEM) has opened doors to integrating these lower-voltage end-users in data-enabled operation and electricity markets. For such implementation SCADA needs to be enhanced and extended to enable participation of even small Distributed Energy Resources (DERs), as shown in red dotted lines in Figure MIT-2. This enhanced end-to-end SCADA architecture is also conceptually based on DyMonDS [14].

\subsubsection{Physical constraints in TEM}

For a TEM approach to work, market transactions must be physically implementable. Bids must be physically implementable, nodal real reactive power must balance, and grid and component constraints must be met. This leads to the following basic constraints which must be accounted for:

\section{a) Market transactions cleared by the TEM must be physically implementable.}

Each DER $i$ can be characterized by the physically implementable relation between incremental reactive power $\Delta \mathrm{Q}$, incremental real power $\Delta \mathrm{P}$ and incremental voltage $\Delta \mathrm{V}$ at time sample [n]. This relation is referred to as a generalized droop.

$$
\alpha[n] \Delta P[n]+\beta[n] \Delta Q[n]=\Delta V[n]
$$

This relation must be accounted for when bids are created with the sub-objectives specific to different market participants.

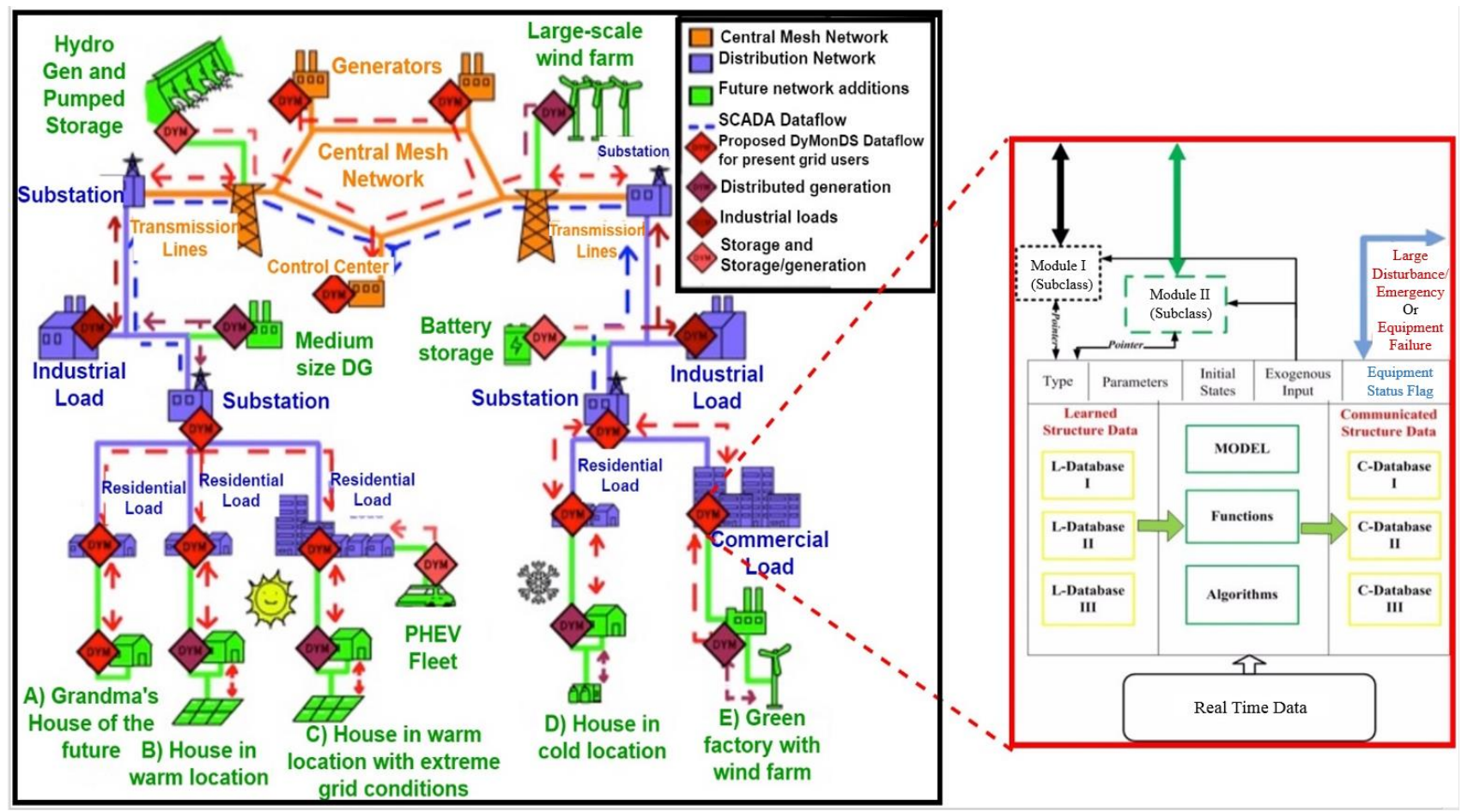

Figure MIT-2 DyMonDS framework [14] 
This generalized droop is a quasi-static characterization of a component resulting from component dynamics in a closed loop, under the assumption that the primary (local) control stabilizes component dynamics in between time samples [n]. For detailed treatment and fundamentals of this relation, see $[4,15]$. Observe that the transaction characterization given in Eqn. (1) is a physically implementable version of a general relation between these three variables, identified in [16, Eqn. 2]. Here, it is derived by combining the internal technologyspecific dynamics and its local control. The local control must be designed so that Eqn. (1) holds at each discrete time sample [n]. In our group, we have recently derived these generalized droops for several DERs, in particular water heaters (WHs), Heating, Ventilating and Air Conditioning (HVAC) units, solar Photo-Voltaics (PVs), batteries and Electric Vehicles (EVs). The basic idea of TEM is to have large numbers of such DERs participate in power balancing and voltage control at low voltage (LV) levels, and therefore to partially offset the need for conventional large-scale generation performing these functions.

The coupled multi-dimensional representations of these droops for thermostatically-controlled loads and inverters are shown in Figure MIT-3(a) and MIT-3(b). The slopes $\alpha[n]^{-1}, \beta[n]^{-1}$ are dependent on the primary controller gains and the present economic dispatch. Often these slopes are less sensitive to operating conditions when controller gains dominate the effect of the inertia of the device. Solar PVs have very low inertia and are dependent on operating conditions. In contrast, these slopes remain constant for the thermostatically controlled devices which inherently have large inertia. It can also be seen from Figure MIT-3 that the inverters are very sensitive and can result in large voltage deviations for small changes in real and reactive power values.

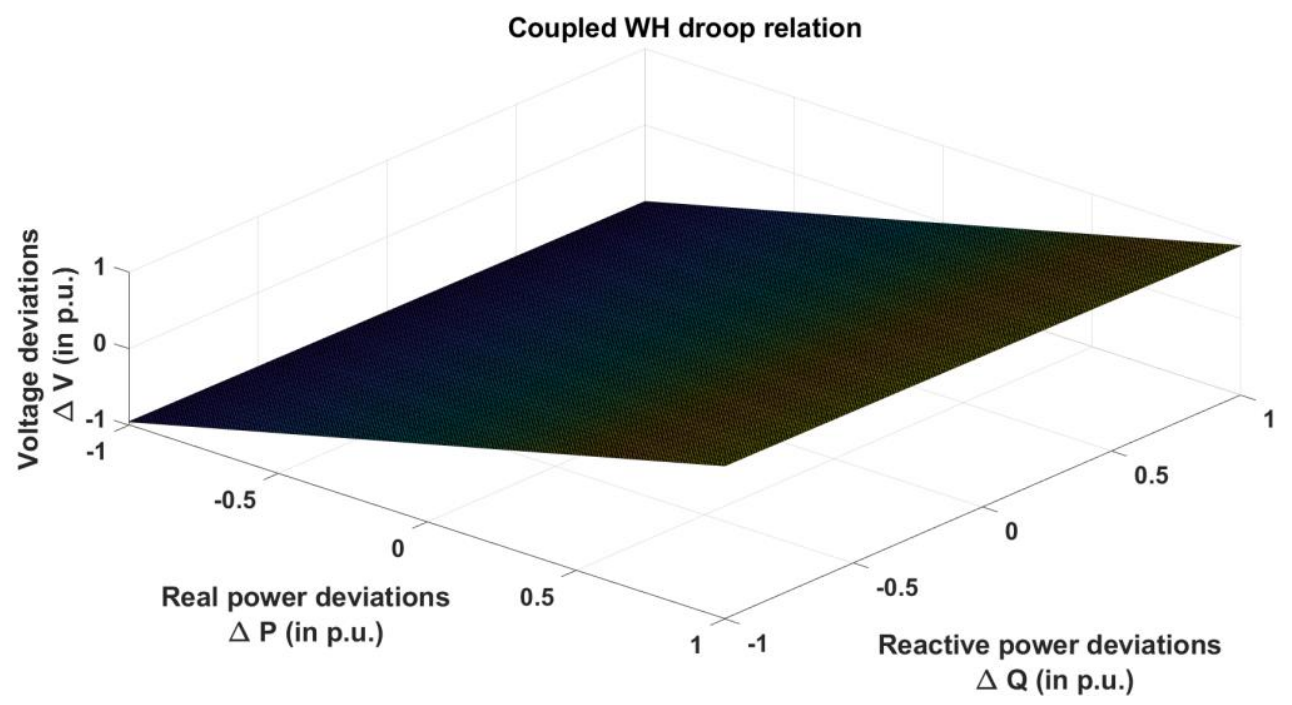

Figure MIT-3(a) Coupled droop of a water heater depicting the three-way relation 


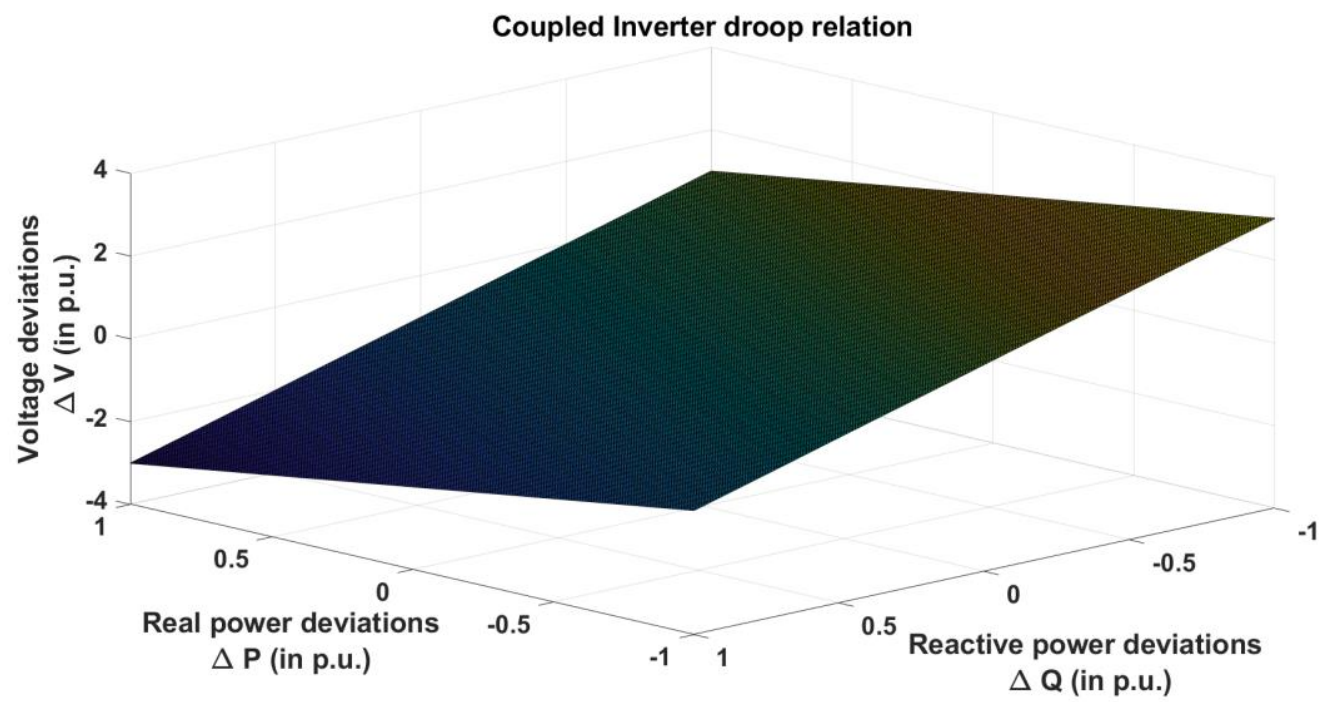

Figure MIT-3(b) Coupled droop of an inverter depicting the three-way relation

b) Both real and reactive power flows must balance at all grid nodes (power flow equations).

$$
\begin{aligned}
& P=A_{p} F_{p} \\
& Q=A_{q} F_{q}
\end{aligned}
$$

Here $P$ and $Q$ are vectors of real and reactive power injections into the grid nodes; $A_{p}$ and $A_{q}$ are matrices relating injections and vectors of real and reactive power line flow vectors $F_{p}$ and $F_{q}$, respectively. Any TEM implementation must observe these grid and power flow constraints.

c) All voltage magnitudes at grid nodes must be within the pre-specified limits.

$$
V^{\min } \leq V \leq V^{\max }
$$

Here $V^{\min }, V$, and $V^{\max }$ are vectors of minimum, actual and maximum nodal voltages. $V^{\min }$ and $V^{\max }$ are defined by ANSI C84.1 standard [17]. Important for the findings in this report is that historically the voltage ranges recommended by this standard assume unidirectional flows; DPS grid equipment and transformer/capacitor regulation settings are generally pre-programmed so that the voltage ranges on the delivery grid do not exceed the low voltage limits at the end-users side that are defined by the ANSI C84.1 standard. In other words, in the past, DPS voltages were required to be such that customer voltages were within this standard [17]. However, the DPS equipment is not necessarily controlled to ensure that ANSI standard is observed at the consumer level. This becomes even more critical in the TE market setting, and it becomes imperative to have TE protocols supporting voltage regulation in addition to balancing power.

\subsubsection{Change of paradigm}

The basic approach to designing equipment and infrastructure needed for regulating voltage profiles in DPS, as well as, more generally, for ensuring that all technical constraints, equations (1)-(3), are met, has begun to quietly change. Depending on the industry organization (rules, rights and responsibilities (3Rs) [18]) and also depending on diverse DER technologies being 
integrated in the existing DPS, new non-unique options for enabling physical performance by attempting to meet technical constraints (1)-(3) have emerged. These changes are based on the observation that technical performance of DERs characterized in terms of their generalized droops (Eqn. (1)) can greatly affect how well network flows are balanced (Eqn. (2)) as well as grid voltage profiles (Eqn. (3)). For example, papers have been written claiming that grid congestion (both voltage and thermal) ${ }^{3}$ can be controlled by the end users themselves [6]. Only recentlyhave we realized that high numbers of dynamically-responding end-users could also contribute to grid performance, in particular to satisfying technical constraints given in Eqns. (2) and (3).

Important for this report is that, depending on how constraints in Eqn. (1) - Eqn. (3) are met, the technical and economic performance could be vastly different. One way of thinking about TEM is that it represents a non-unique control and market mechanism for enabling physically implementable performance. However, the performance greatly depends on whether DERs are price takers (Cases 1-3 in this report) or price makers (Case 4). A more technical observation is that the performance will also depend on whether the TEM implementation is based on feedforward or feedback signals. A typical DER (WH, HVAC or PV) could offer a binding bid in anticipation of prices and other conditions (feed-forward), or these devices could respond to deviations in physical performance (temperature, for example) which is a result of scheduling. More generally, a feed-forward TEM approach implies that bids are created so that the voltage constraint given in Eqn. (3) and/or physical performance of DERs given in Eqn. (1) are accounted for when the decision is made (the MIT feed-forward approach) or after the bids are implemented (feedback). The latter means that a bid can be created without observing its voltage ranges, and then the local DER controller would respond to voltage deviations outside of allowable voltage ranges by adjusting its real and/or reactive power consumption. Similarly, the grid operator and TEM market clearing mechanism could optimize bid selection so that voltages are maintained within the limits (MIT approach), or without observing constraints given in Eqn. (3) and just ensuring power flow balances given in Eqn. (2) [19]. Depending on the way voltage is managed, the voltage violations may take place or not. In this report, NETSS optimization software is used to ensure such violations do not occur. These different combinations need to be clearly understood when 3Rs for well-functioning TEM are set, since they require qualitatively different TEM infrastructure complexity and cost, and result in different overall performance. In our report we illustrate these implications on the IEEE 8500 DPS. This test system is analyzed as the case in which SCADA processes medium voltage (MV) economic signals, and local infrastructure between MV nodes and the relatively small number of the LV households directly connected to the MV nodes by neglecting the single-phase distribution wires that form the network downstream of the MV nodes.

\subsubsection{Distributed decision making for TEM}

The ultimate objective of TEM should be to enable participation of DERs at value, and to support well-defined system performance metrics. The actual implementation is distributed with some sort of minimal coordination. Distributed decisions are supported by sub-optimizations of all agents (DERs, grid operators and TEM market clearing mechanisms), all interacting to align their sub-objectives with the system objectives. This very general TEM problem statement leads

\footnotetext{
${ }^{3}$ In this report, the emphasis is on voltage problems. In addition to voltage constraint (Eqn. (3)) one can include thermal line flow limits. However, these are only observed at HV/MV grid levels. LV levels have fuses which disconnect equipment if needed.
} 
to non-unique outcomes depending on many factors. TEM market clearing mechanism concerns are mainly economic outcomes at the system level (system social welfare defined as the difference between expected benefits to end-users and cost of power to serve the end-users: individual profits, benefits, revenues); the grid operator is concerned with technical constraints so that quality of service (QoS) to the end users is achieved. Finally, the DERs have their own sub-objectives, such as consumers maximizing their benefits, subject to internal constraints (such as comfort) for anticipated internal needs and in response to and/or in anticipation of external signals (price of electricity, weather) or constraints.

Each agent (appliance, household energy manager; aggregator) makes data-enabled decisions based on the predicted, learned or historical knowledge about exogenous signals, both physical (weather, grid state) and economic (prices). Each agent has its own objective and internal constraints. However, there are several aspects of TEM that are extremely relevant for understanding differences in outcomes. They are briefly discussed next. For more mathematical treatment, see [20].

\subsubsection{Non-unique performance objectives: the key role of voltage optimization and predictions}

In wholesale markets, the typical market-level objective is short-term social welfare. Given generation and demand bids, the market is cleared so that short term outcome is optimized. The optimization is done so that network constraint (2a) is met and decision variables are real power injections. This optimization is done using DC OPF by today's ISOs. However, the most efficient cleared bids are often further modified in coordination with the grid operator so that network constraint (2b) and voltage constraints (3) are met. At present, no AC OPF is used, and because of this, bid modifications for ensuring physical grid implementation are sub-optimal.

TEM is mainly intended to enable distribution level end users' participation in what one might think of as retail markets. The TE Challenge Phase 2 primarily concerns cases in which DERs create voltage problems. Because of this, it is extremely critical to set protocols with well understood 3Rs for voltage management at value. Most of our simulations are intended to stress this aspect of retail markets. Ultimately, TEM should give sufficient incentives to utilities to participate at value and not consider the grid as a hard constraint to the DER participation; that is, to include customer DER flexibility in hosting capacity analysis. To begin to think in this direction, it is easier to consider the case in which the TEM market maker is at the same time a grid owner. When this is not the case, there are further complications.

To assess these issues, let us think first of performance objectives as being different with respect to the time over which decisions are being made. Some envision major benefits from peer-topeer long term cooperation for investments in DER technologies, with the short-term optimization effects having secondary effects [21]. However, most of TEM work is currently targeted to short-term optimization, as described in this report. This approach assumes that peerto-peer cooperative longer-term contracts are financial and not physical, and, as such, the longterm signals are not included in the TEM computer platform. Nevertheless, the actual cost and benefit functions used by agents often include, explicitly or implicitly, the levelized investment cost. If this is not done, at most many DERs would have to bid zero price. It is well known and has been documented that this leads to real problems with sustained businesses, including future 
investments in new technologies [21]. These investment-related aspects of TEM are not discussed here, but should probably be a major topic for future work. Only if levelized cost functions are allowed in bidding protocols and/or a market supports differentiated reliability/resiliency of service, does it become possible to have sufficiently strong economic signals to incentivize deployment of TEM infrastructure at value. Related, the TEM performance greatly depends on the time horizons over which decisions are being made. In general, the longer the time horizon, the more optimal the performance is. In operations, this means it is better to optimize in a model-predictive way. Aggregation of DERs at different levels supports cooperation among DERs, which can be shown to reduce overall market complexity. It also increases the value brought about by active participation. An important open question concerns how to relate decision making outcomes over multiple time horizons. The idea of "distributed dynamic investments" in TEM infrastructure at value, by which potential risks brought about by various uncertainties is reduced by distributing it over many different cooperative DERs and over the lifetime of infrastructure needs further studies. Market mechanisms for incentivizing aggregation of groups of DERs for cooperatively participating in TEM remains an open problem at present. Tradeoff analysis between the investment cost for embedding intelligence in these DERs and the saved energy bills through such participation needs to be assessed.

\subsubsection{Optimization subject to hard or soft constraints}

Another important aspect of TEM concerns how different hard constraints are modeled when different stakeholders perform their functions. For example, the technical and economic performance will differ significantly depending on how the comfort constraints are modeled by the DERs during their TEM participation. For several formulations of decision making by different stakeholders and illustrations of their differences depending on how constraints are modeled, see [15]. Most important is our finding that the ability of DERs to participate in TEM is affected by this choice of hard vs. soft constraints. We illustrate these aspects of TEM performance on the test system later in this report.

Soft constraints could be imposed either internally by the distributed agents themselves, or through the binding commands within the TEM. Important for understanding potential TEM outcomes is to be aware of this subtle difference. We point out that TEM outcomes (technical and economic) are different when the voltage constraint given in Eqn. (3) is included in DER's sub-optimization, or when it is not. If not included, either grid operator/TEM market mechanism can perform optimization subject to all voltage constraints (using AC OPF, for example), or the TEM could result in voltage excursions outside those specified by the ANSI C84.1 standard. Even more intriguing is the fact that TEM can either use the results of its AC OPF optimization to create or not create a reactive power price signal, which may serve as an additional incentive signal for DERs to adjust their reactive power consumption. Depending on what is being done, the results are very different. Similar issues arise when DERs create their bids depending on whether they consider their own comfort limits to be hard constraints or not. It will be shown in 5.5.1.1 how the bids of different DERs as distributed decision makers change when different relaxations of the comfort constraint are utilized. 


\subsubsection{TE Challenge Use Cases and Analysis}

The four TE Challenge use cases have been presented above in the NIST overview. These use cases are reviewed below along with implications for how the use cases may be implemented and impact results.

\subsubsection{TEC Case 1}

TEC Case 1 is a baseline case where the generation schedule is obtained for a combination of uncontrolled solar PVs and plug-loads operating according to user demands. Appliances such as WHs and HVACs consume on an as-needed basis. The basic role of PVs is to provide as much clean solar power as possible to households without participating in grid control. No embedded software is used by the DERs since no adjustments are expected by the grid and/or market. Resulting benefits and costs seen by households are a direct consequence of power consumed, available solar power and flat electricity tariffs. Households are not decision makers. On the other hand, the local distribution grid plays a major role as it is primarily responsible for the Quality of Service (QoS), such as frequency and voltage, and for power delivered to the households. In the test system used in this report, we illustrate major implications of the performance objective used by the distribution grid when providing electricity service. We show that a distribution operator can also proactively participate in controlling voltage and ensuring that the voltage profile is within the ANSI C84.1 standard expected by the end users. An AC OPF can be used to optimize the voltage profile by controlling voltage regulating devices such as shunts and regulators, such that the MV level voltage remains within a voltage range that ensures end users' voltages remain within the required standards.

Our assessment of TE cases is done by assuming there exists a load serving entity at each of the MV nodes that further interacts with the LV end-users. However, the model neglects the single phase $7.2 \mathrm{kV} / 240 \mathrm{~V} / 120 \mathrm{~V}$ network's effect on economic transactions. In case voltages are unacceptable, or other operating grid problems occur at times of excessively high or low irradiance, it becomes necessary to provide incentives to the distribution grid to invest in regulation. Tariffs are needed that give incentives to the distribution grid to do this efficiently. The utilities remain key to enabling TEM and should be compensated at value brought to the system. At present such incentives do not exist, and utilities are threatened by the distributed resources. Industry stakeholders require methods to determine value of new grid investments (in both hardware and software for smart hardware), versus additional renewable power. Because these incentives are lacking, what one may consider to be a baseline least cost is actually not. This is primarily because the solutions are not physically implementable, as they typically result in violation of ANSI C84.1 standards. In addition to this, if solar power is high this may result in significant reverse power flows and the distribution grid will need different control and protection to enable these flows.

\subsubsection{TEC Case 2}

Case 2 is similar to Case 1, except that during a storm solar irradiance is suddenly reduced. Since household appliances do not respond, more power needs to be delivered from the substation. Here again the distribution grid control becomes important; by regulating substation and other MV, as well as power sent from the substation, this becomes possible without violating ANSI C84.1 standards. Retail market mechanisms need model-predictive control (MPC) to minimize the risk of operating problems during storms. As part of our on-going ARPA-E NODES project 
we have assessed potential benefits from replacing static optimization with MPC optimization $[19,26]$. We illustrate some of these retail market MPC methods in the analysis to follow.

\subsubsection{TEC Case 3}

This is the case of households being price takers. Given TOU price, households become proactive decision makers with the objective of minimizing their energy bills. Their performance sub-objective is to minimize the expected electricity cost while, at the same time, ensuring that their own comfort is met. Several variations of this distributed optimization by the end users are possible. In particular, optimization could be done by modeling comfort as a hard constraint or as a soft constraint. A soft constraint could result in unacceptable performance of WHs or HVACs, but could save on the energy bill. Since cost is of concern over a longer time, an effective DER energy optimizer should be MPC-based. An intriguing aspect of TEM decisions is the fact that any agent will experience higher local efficiency if, in addition to real power consumption, it also optimizes the reactive power consumed $[4,15]$. Moreover, the real and reactive power consumed by the appliances will depend on whether the voltage constraint given in Eqn. (3) is accounted for. The end users decide on the minimum and maximum power they need and tell their aggregator (at MV node). The aggregator, in turn, communicates the aggregate of DER bids to the distribution grid and/or retail market. The TEM protocol for Case 3, when end-user consumption is expected to depend on TOU price, must also specify which agent is responsible for voltage support. If it is the end user, then the distribution grid will not include this in its objective when defining TOU tariffs. Otherwise, if the distribution grid has the role to support voltage, this cost must be included into tariff. This is to say that TOU protocols are needed to clearly define roles and responsibilities of grid users, and the grid itself. Studies are needed to compare these solutions.

\subsubsection{TEC Case 4}

This case involves dynamic pricing and it comes the closest to a win-win TEM protocol. The end users and their MV aggregators create bids in anticipation of electricity prices, and, at the same time, they affect them (price makers). In some sense one can think of this case as being fair, since all TEM participants can be decision makers according to their own sub-objectives. There are several variations here as well depending on roles and responsibilities of TEM participants. In contrast with Case 3, electricity price is affected by the protocol details. The end users could both try to minimize their electricity bill, maximize the utilization (efficiency), control their voltage or not. Similarly, the distribution grid has various versions. We show that given any of these versions, one can relate the price signals to the roles and responsibilities. For example, if end users do not control their own voltage, the price will include a component that reflects value of voltage support by the grid. To do this well, distribution grids need AC OPF with the ability to control voltage. We illustrate the use of AC OPF for this purpose, and potential benefits from doing this. In this case it becomes most obvious that there exist definitive tradeoffs between the complexity of decision making by the TEM participants and the overall TEM performance.

Major observation: neither Case 1 nor Case 3 are good baseline cases against which TEC Case 4 performance should be assessed. As described above, although Case 1 appears to be the lowest cost case, generally it is not physically implementable. Similarly, TEC Case 3 generally does not have an easy-to-defend time of use tariff. Only Case 4 provides a basis for much innovation and distributed choice. We next illustrate these cases on the IEEE 8500 distribution feeder system. 


\subsubsection{Roles and performance metrics of different TEM participants}

Shown in Table MIT-1 is an illustration of roles and performance metrics of TEM participants for the four TEC cases.

Table MIT-1 Roles and Performance metrics in TEC cases

\begin{tabular}{|c|c|c|c|c|c|c|c|}
\hline \multirow[t]{2}{*}{ Case } & \multicolumn{2}{|c|}{ End-users } & \multicolumn{2}{|c|}{ Distribution Grid } & \multicolumn{2}{|c|}{ Market } & \multirow[t]{2}{*}{ Notes } \\
\hline & Role & Sub-Objective & Role & Sub-Objective & Role & Sub-Objective & \\
\hline $\begin{array}{l}\text { 1. normal } \\
\text { sunny } \\
\text { day }\end{array}$ & Passive & $\begin{array}{l}\text { utilize solar } \\
\text { power }\end{array}$ & $\begin{array}{l}\text { Critical, } \\
\text { including } \\
\text { possible reverse } \\
\text { flows }\end{array}$ & $\begin{array}{l}\text { feasible power } \\
\text { delivery; ANSI } \\
\text { C84.1 }\end{array}$ & minimal & $\begin{array}{l}\text { Economic } \\
\text { efficiency }\end{array}$ & $\begin{array}{l}\text { Operating } \\
\text { problems time- } \\
\text { varying. } \\
\text { Baseline case. }\end{array}$ \\
\hline $\begin{array}{l}\text { 2. Storm } \\
\text { in the } \\
\text { middle of } \\
\text { Case } 1\end{array}$ & Passive & $\begin{array}{l}\text { have power } \\
\text { during both } \\
\text { normal } \\
\text { conditions and } \\
\text { during storm }\end{array}$ & $\begin{array}{l}\text { Role of } \\
\text { distribution } \\
\text { grid--critical }\end{array}$ & $\begin{array}{l}\text { Sub-objective - } \\
\text { feasible power } \\
\text { delivery; ANSI } \\
\text { C84.1 }\end{array}$ & minimal & $\begin{array}{l}\text { Economic } \\
\text { efficiency }\end{array}$ & $\begin{array}{l}\text { Potential } \\
\text { problems } \\
\text { during storm }\end{array}$ \\
\hline $\begin{array}{l}\text { 3. Time- } \\
\text { of-Use }\end{array}$ & $\begin{array}{l}\text { decide } \\
\text { on } \\
\text { power } \\
\text { to use }\end{array}$ & $\begin{array}{l}\text { Minimize } \\
\text { energy bill; } \\
\text { maximize local } \\
\text { efficiency; } \\
\text { ensure comfort; } \\
\text { support voltage } \\
\text { or not. }\end{array}$ & $\begin{array}{l}\text { less critical; end } \\
\text { users could } \\
\text { support their } \\
\text { voltage; } \\
\text { participate in } \\
\text { delivery }\end{array}$ & $\begin{array}{l}\text { feasible, } \\
\text { physically } \\
\text { efficient delivery; } \\
\text { if supporting } \\
\text { voltage, build } \\
\text { smart } \\
\text { infrastructure }\end{array}$ & minimal & $\begin{array}{l}\text { Economic } \\
\text { efficiency }\end{array}$ & $\begin{array}{l}\text { Could be } \\
\text { conflicting } \\
\text { sub-objectives; } \\
\text { Need for } \\
\text { voltage support } \\
\text { protocol }\end{array}$ \\
\hline $\begin{array}{l}4 . \\
\text { dynamic } \\
\text { electricity } \\
\text { pricing }\end{array}$ & $\begin{array}{l}\text { decide } \\
\text { on } \\
\text { bidding }\end{array}$ & $\begin{array}{l}\text { Minimize } \\
\text { energy bill; } \\
\text { maximize local } \\
\text { efficiency; } \\
\text { ensure comfort; } \\
\text { support voltage } \\
\text { or not. }\end{array}$ & $\begin{array}{l}\text { less critical; end } \\
\text { users could } \\
\text { support their } \\
\text { voltage; } \\
\text { participate in } \\
\text { delivery; MUST } \\
\text { GIVE SIGNAL } \\
\text { TO MARKET }\end{array}$ & $\begin{array}{l}\text { feasible, } \\
\text { physically } \\
\text { efficient delivery } \\
\text { AT VALUE; if } \\
\text { supporting } \\
\text { voltage, build } \\
\text { smart infra- } \\
\text { structure; GET } \\
\text { PAID FOR THIS }\end{array}$ & $\begin{array}{l}\text { Critical; } \\
\text { create } \\
\text { dynamic } \\
\text { prices which } \\
\text { reflect not } \\
\text { only real } \\
\text { power but } \\
\text { also } \\
\text { VOLTAGE }\end{array}$ & $\begin{array}{l}\text { Efficient } \\
\text { market clearing } \\
\text { mechanism by } \\
\text { considering } \\
\text { bids at value } \\
\text { from both end } \\
\text { users and grid }\end{array}$ & $\begin{array}{l}\text { Potentially } \\
\text { win-win fair } \\
\text { case }\end{array}$ \\
\hline
\end{tabular}

\subsection{Computer Simulation Platform and Scenarios}

In this section we first describe the MIT SEPSS computer platform used for TEM simulations. This is followed by the description of basic TEM simulation scenarios analyzed.

\subsubsection{SEPSS Computer Simulation Platform for TEM}

We utilize the SEPSS co-simulation platform for emulating transactive energy markets. The SEPSS user logs into the MIT-LL Supercloud through the login node and then activates the SGRS scheduler on one of the compute nodes. The SGRS scheduler is a BSD-3 licensed software developed at CMU that allocates the computing resources provided by the MIT-LL Supercloud in such a way that the inherent structure in power system topology and the hierarchies involved are explicitly accounted for [17]. The SEPSS platform shown in Figure MIT-2 is a more general architecture, while the one in Figure MIT-4 shows a specific information exchange framework overlaid on top of the computer platform, and used to demonstrate TEM concepts. 
Shown over the black colored arrows is the information that gets communicated only at the beginning of the simulation for allocating the computing resources alone. The scenario and configuration file of interest are fed to the scheduler by the client through the Supercloud's login node. The administrator of the platform provides a list of available nodes, which are then utilized by the scheduler to instantiate objects in the scenario file and allocate dedicated compute nodes for each or several of the object instances. In doing so, to minimize the latency, the hierarchical structure in power systems is duplicated onto the computer platform. For instance, the neighborhood coordinates several DERs within the houses. Hence, objects of neighborhood types are instantiated on a Supercloud compute node, which in turn instantiates several other house objects on several processors that constitute this compute node. The dense cluster node is allocated for objects of neighborhood type as shown in blue colored rectangles. The details of the information that these objects communicate are shown on top of blue-colored lines.

The cluster nodes further allocate processors within the cluster for simulating the houses that constitute the neighborhood. These are shown as orange colored instances, the detailed information exchanges within which are shown on top of the red colored lines. The MIT-LL Supercloud architecture allows for a maximum of 16 processes on each compute node. One or several houses belonging to a neighborhood get instantiated on each processor. In case the number of houses within a neighborhood is larger than 16 , the houses get instantiated on two separate compute nodes and these nodes are grouped. If the number of houses is much smaller, several neighborhoods get instantiated on the same compute node. Unique to MIT's platform is that once the resources are allocated, there is no requirement for a master program or a simulation orchestrator to coordinate the flow of simulation.

Finally, a dedicated node shown in grey is allocated for instantiation of the ISO module, which consists of several sub-modules such as the weather forecast and grid modules. Here the ISO module coordinates several neighborhoods by also accounting for the grid that connects them. The weather forecast and prices are sent out by the ISO module to the neighborhoods. This information is further sent to the house instance within. The consumer sub-module within each of the houses, utilizes weather and the historical consumption to predict the future consumption requirement of each of the devices. It must be noted that the price forecaster modules may reside at the ISO level or at the medium voltage aggregator level communicating system level and neighborhood-level anticipated prices, respectively. DERs choice of receiving weather forecasts is non-unique. There may be dedicated entities embedded at MV aggregators and ISO level for this purpose. 


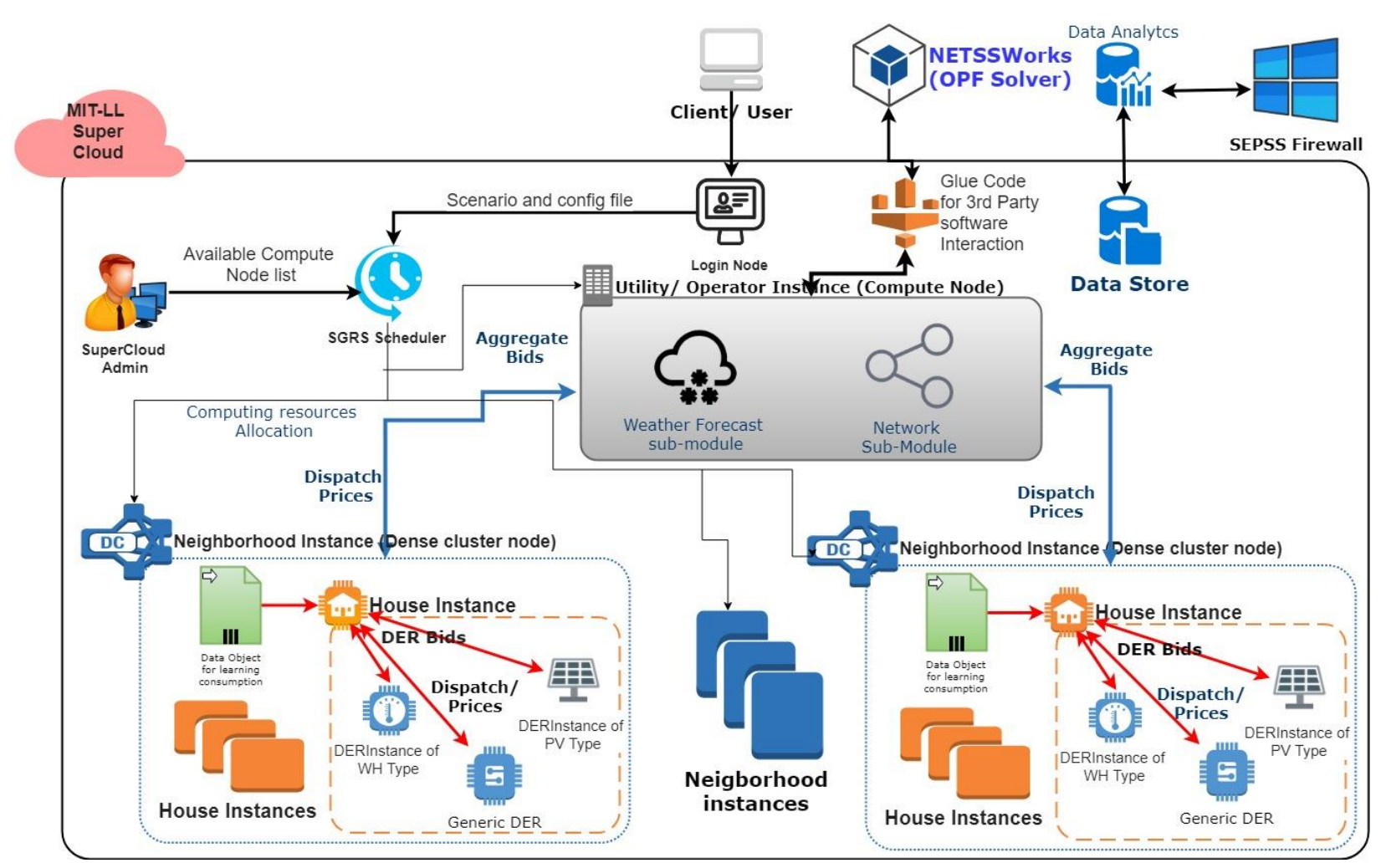

Figure MIT-4 Computer architecture for simulating the interactions needed for enabling TEM

For TEC Cases 3 and 4, the device modules utilize this information to compute the optimal electrical consumption requirement along with the price elasticity (for Case 4) and send these to the neighborhoods. The price elasticity gets computed by finding the real and reactive power consumption values for anticipated real and reactive power prices by solving the DER-level problem. The same problem is solved four more times for perturbations in real power and reactive power prices, which then allows one to obtain sensitivity with respect to each of these prices. For more details on sensitivity-based bid creation, please refer to [22, 24]. The neighborhood coordinator further utilizes this information to compute the aggregate consumption requirement and the aggregate price elasticity (for case 4) to the ISO. The ISO, with the help of the embedded load forecaster that gives an estimate of inflexible plug load, clears the market and sends out the dispatch quantities and cleared prices to the individual neighborhoods, which finally get communicated to the individual DERs based on their price bids. Such information exchange is simulated every 10 minutes and, furthermore, the intelligence embedded in DERs is such that it also computes optimum real and reactive power adjustments every 2 minutes so that voltage violations are minimal, see $[15,20]$. Note that in Cases 1 and 2, the lower level neighborhood modules are not instantiated, and the total load and PV injections are treated as inflexible, the values of which get computed by the load forecaster using an AC OPF algorithm.

\subsubsection{Basic TEM Simulation Scenarios Setup}

While the ultimate goal of our simulation setup is to analyze and compare TEC Cases 1 through 4 with regard to the technical and economic performance metrics of interest in this challenge, it is important to set these cases so that they can be seen as the specific cases of the general 
information flow shown in Figure MIT-4. The four TEC cases differ with respect to the specific economic and technical signals exchanged, as well as with respect to the decision- making objectives embedded in different stakeholders. The relevant economic signals are computed over longer periods of time to support market processes; at the same time the technical metrics must be observed in near real time, since the feasibility of market outcomes must be ensured in an operationally implementable way.

We observe that the decisions are made in a highly uncertain environment. Depending on the market protocols and algorithms used, these uncertainties are accounted for in different ways and have different implications on TEM outcomes. In our simulations, DERs (including solar PVs) are grid users and they generally manage uncertainties in a highly distributed way given minimal system-level coordination. The grid and system operator clear the bids using static AC OPF. In our co-simulation setup external software called NETSSWorks is used for market clearing so that the grid constraints are observed [25]. This specific software is particularly suited for the scenarios in which grid voltage constraints may be the limiting factors to the number of solar PVs and other DERs able to participate. Instead of scheduling DERs and running power flow analysis to compute the effects of their TEM participation on grid's ability to serve small low voltage (LV) households according to ANSI C84.1 standards [17], a computationally robust AC OPF is used to ensure that the DERs, utility generation and controllable grid equipment (capacitors, reactors) have set points for their controllers to ensure that the operating limits are observed while attempting to optimize system-level metrics. NETSSWorks selects specific performance metrics in an automated way depending on the objectives of interest and the grid ability to operate within the given constraints. Notably, voltage set points of controllable grid equipment and controllable DERs are optimized so that system-level optimal power flow (OPF), optimal reactive power flow (OQF), optimal apparent power (OSF), optimal load dispatch (OLD) or optimal achievable voltage profile (MXV) are computed. Employing AC OPF instead of power flow at the market clearing using DC OPF enables one to optimize dispatchable components for both voltage and reactive power.

The TEM bids offered by the DERs require that they predict and/or learn their consumption patterns and preferences, as well as likely weather conditions and make decisions in a MPC manner [6,22]. Fundamental to the MPC-based decision making is that while the optimization time horizon is long in the future, only the bid for the next market clearing time is communicated. This MPC-based approach is fundamental to the DyMonDS framework and SEPSS implementation. It enables choice and distributed risk management typical to market environments.

In what follows we illustrate the effects of different decision-making algorithms and information exchanged, within a TEM in context of Cases 1 through 4, on both economic and technical outcomes. The simulations are particularly targeted to assess voltage-reactive power management protocols. Shown in Table MIT-1 is a summary of roles and sub-objectives for both grid users and for grid/system/market operators. To understand the role of different stakeholders in an environment with many solar PVs, it is important to consider the voltage-reactive power aspects of operation carefully. We point out that there exist qualitative differences between: (1) using power flow analysis algorithms for assessing voltage profiles resulting in scenarios studied; and, (2) using an AC OPF capable of optimizing real power dispatch and voltage dispatch within the acceptable operating limits so that the performance objectives of interest are 
optimized. All our simulations use AC OPF NETSSWorks software co-simulated with SEPSS, as shown in Figure MIT-5 to perform (2). A typical example of benefits from using AC OPF which optimizes both real power and voltage dispatch rather than power flow (or DC OPF; or AC OPF subject to voltage constraints), is its use to enable a feasible power delivery within the acceptable voltage limits, while, at the same time, supporting higher DER participation. The implementation of voltage optimization can be done either by: (1) the distribution system by optimizing capacitor/reactor set points; or (2) the grid users (DERs, PVs) controlling their own consumption so that their local voltages are within the limits. Many technologies exist for such control, for example PVs can use inverter control, HVACs can use variable speed drives and even water heaters can have small controllable local capacitor/reactor shunts at the household level. In short, the grid optimization software coupled with MPC-based bid creation algorithms embedded in participating DERs support TEM implementation so that the grid users' objectives are aligned with the grid system objectives. The economic signals provide adequate market incentives.

\subsubsection{Voltage Regulation in TEM}

Depending on the particular TEM protocols in place, the method for ensuring voltage regulation according to ANSI C84.1 standard is generally non-unique. Even more striking is that the economic incentives for doing this can be quite diverse, as discussed later in simulation results. Perhaps somewhat unique to the MIT TEM approach is that the participating DERs have embedded MPC-based distributed decision making whose objective is to minimize a combination of their energy bills and/or the energy conversion inefficiencies unique to their technologies and which is subject to local voltage limits. At the same time, the consumer comfort requirements must be met either at fast and/or slow time scales. See [6,20] for more details on derivations of a new technology-specific "droop" relating real power, reactive power and voltage of the device in closed-loop. As a result, each TEM agent submits the sensitivity of its cost function with respect to the real power consumption (real power price bid) coupled with the time varying minimum and maximum real and reactive power limits. See [15, 20] for more details on the TEM design. These sensitivities of all devices incident directly or indirectly through a network onto a MV node are aggregated to compute the net sensitivities of consumption costs with respect to real power consumption (MV level real power price bids) and the total real and reactive power limits that the MV level aggregator offers to the wholesale market.

The system operator, on the other hand, utilizes these aggregate bids to then dispatch the DERs for the next time instant such that the voltages at each of the buses are ensured to stay within the band of 0.95 p.u. to 1.05 p.u., which is the worst case operating voltage after accounting for the LV distribution feeder voltage drops so that adherence to ANSI standards of distribution level voltage are assured (explained later in simulations). It must be noted that the worst case allowable voltage deviations are committed by the TEM agents over a slower market time scale in a feedforward way through real and reactive power bids, and it is the DER's responsibility to ensure through local control that voltage deviations do not exceed the value committed to in the market.

Another possibility is for the network to assume the responsibility of ensuring that voltage violations at the market time scales remain bounded. This requires adjusting the set points of automatic voltage regulators and shunt capacitors/reactors to which fast local feedback control reacts. Later in this report we discuss the implications of these different protocols for regulating 
voltage and their relations to utility tariffs. Note that this latter approach is utilized for ensuring implementable TEC cases 1 and 2, while there is an option to choose either approach for TEC Cases 3 and 4.

\subsection{Test System Analysis}

In this section we consider a synthetic system example created so that it resembles operating issues with high solar penetration. The analysis is based on the data created by PNNL, which is available as open source data [26]. The starting system is the standard IEEE 8500 bus distribution feeder with a static snapshot consumption. Random time-varying data is produced by GridLAB-D using a random seed data generator to ensure reproducibility, which replaces static consumption by the time-varying residential loads, see details in [19, 26]. For the purpose of showing the effect of TE, we neglect single phase distribution wires downstream of the MV network and make an assumption that all the houses are directly incident on MV nodes. With such an assumption, we simulate the TEM. We later perform the analysis to assess if the LV nodes' voltages are within the ANSI C84.1 limits. Based on the worst-case voltage drop that may occur in the distribution wires, tolerable voltage limits for MV nodes can then be set. Figure MIT-5 shows the net aggregate load composition and solar power seen at the substation level. The changes in the uncontrollable component and the solar radiation changes drive the TEM.

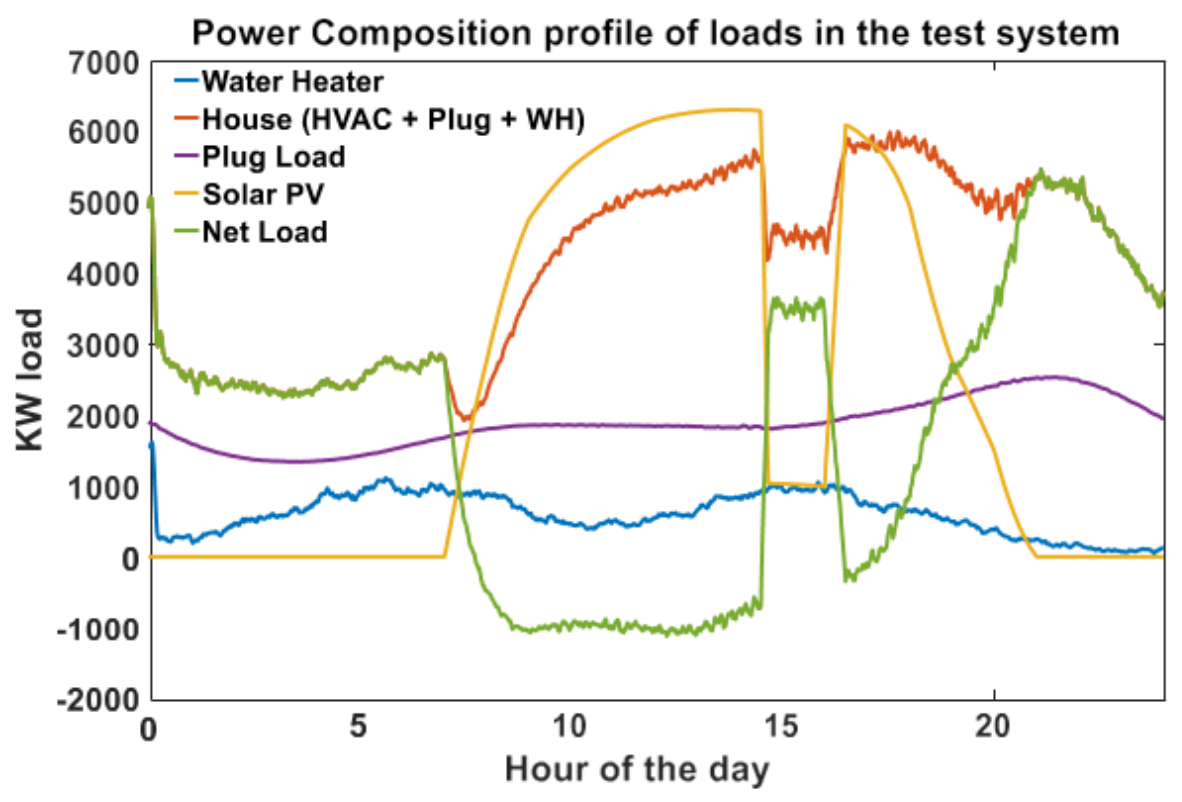

Figure MIT-5 Time series of the DER consumption patterns (negative consumption for solar PV generation) of the IEEE 8500 test system

\subsubsection{Factors affecting $T E$ market outcomes}

We consider a DER control approach (the base case discussed below) where DER-level market bid decisions are made such that comfort is maintained (based on worst case user-demand in the coming interval) across the market bid interval. The algorithms governing this approach are obtained from the physics-based models and are briefly described next. Detailed mathematical formulations can be found in [20]. There are several variations of the slower market signals that DERs can commit to and one must assess which protocol suits best a given grid with different 
composition of DERs. In the rest of the analysis in this section, we consider a variation in which DERs maintain their own terminal voltage by adjusting their real and reactive power injections into the grid.

\subsubsection{Effect of comfort constraints on the elasticity of bids}

We briefly illustrate how the bid function of DERs can change as different variations of comfort constraint relaxations are utilized. Recall the DER droop that relates P, Q and V in Eqn. (1) for typical DERs. However, the market interval is in general much slower than the time scale needed for the validity of Eqn. (1).

In order to homogenize different forms of energy consumed to that of the electrical quantity, we utilize the notion of energy conversion droop to derive the following relation, the details of which can be referred to in [15]. For each market clearing time step $k$, if the comfort quantity of DER is represented by $W[k]$ and its electrical consumption is denoted as $P[k]$, the differences are linearly related through energy-conversion droop $\sigma$ as

$$
\sigma \Delta W^{\min }[k] \leq \Delta P[k] \leq \sigma \Delta W^{\max }[k]
$$

As an example, for thermostatically-controlled devices, the difference in comfort over subsequent time intervals is defined as

$$
\Delta W[k]=\widehat{m}[k](\theta[k]-\theta[k-1))+\theta[k](\widehat{m}[k]-\widehat{m}[k-1)),
$$

where $\widehat{m}[k]$ is the average expected flow rate of water/air in the $k^{\text {th }}$ market interval and $\theta$ denotes temperature. A key observation which becomes critical in such demand participation is that the allowable temperature variation is higher when the set of allowable values of electrical consumption is larger. Since the quasi-static characterization is in terms of increments from the present operating conditions, thus the closer the operating condition $(W)$ is to the tolerable enduse limits $\left(W^{\min }, W^{\max }\right)$, the smaller the band of allowable power consumption adjustments as can be seen from the inequality constraint in Eqn. (4). As a side note, one might consider a combination of storage elements considered as a module to provide wider bands. Furthermore, relaxation 2 (below) may result in zero change in electrical consumption at all times for symmetrical limits of allowable temperature variation. Hence, we impose the constraints in such a way that the allowable maximum temperature deviation is $2.8^{\circ} \mathrm{C}\left(5^{\circ} \mathrm{F}\right)$ above the programmed upper temperature limit and $1.7^{\circ} \mathrm{C}\left(3^{\circ} \mathrm{F}\right)$ below the minimum temperature limit.

Eqn. (4), when used as a hard constraint in the DER formulation, is referred to as the base formulation. This often results in conservative DER dispatch. Hence, a relaxation of the constraint is instead used in the DER level formulation to compute bids. We consider the following two types of relaxations and see qualitatively how the bids differ for each of the demand technologies:

- Relaxation 1: Apply a summation on the inequality constraint in Eqn. (4) for the entire planning horizon. The resulting constraint relates power and comfort over the next few market time steps. The intuition behind such relaxation is that the expected usages over longer time-scales may result in more reliable limits on real power consumption. 
- Relaxation 2: Consider the constraint in the objective function by imposing a unit penalty factor on temperature excursions outside of allowable limits. Such relaxation makes the comfort constraint non-binding which, for slow evolving devices such as WHs and HVACs, will not result in considerable deviation of consumer comfort.

Given the anticipated prices of real $\left(\hat{\lambda}_{p}\right)$ and reactive $\left(\hat{\lambda}_{q}\right)$ power consumption, a general optimization problem posed at the device level for the base case constraint as in Eqn. 4 is as follows (note that there are also other voltage-related constraints that are not shown here in the interest of space):

Base Case:

$$
\begin{gathered}
\min _{P[k], Q[k]} \sum_{k=T}^{T+H} \hat{\lambda}_{p}[k] \mathrm{P}[\mathrm{k}]+\hat{\lambda}_{q}[k] \mathrm{Q}[\mathrm{k}] \\
\text { s.t. } \sigma \Delta W^{\min }[k] \leq \Delta P[k] \leq \sigma \Delta W^{\max }[k]
\end{gathered}
$$

Relaxation 1:

$$
\begin{gathered}
\min _{P[k], Q[k]} \sum_{k=T}^{T+H} \hat{\lambda}_{p}[k] \mathrm{P}[\mathrm{k}]+\hat{\lambda}_{q}[k] \mathrm{Q}[\mathrm{k}] \\
\text { s.t. } \sum_{k=T}^{k=T+H} \Delta W^{\min }[k] \leq \sum_{k=T}^{k=T+H} \Delta P[k] \leq \sum_{k=T}^{k=T+H} \Delta W^{\max }[k]
\end{gathered}
$$

Relaxation 2:

$$
\begin{gathered}
\min _{P[k], Q[k]} \sum_{k=T}^{T+H} \hat{\lambda}_{p}[k] \mathrm{P}[\mathrm{k}]+\hat{\lambda}_{q}[k] \mathrm{Q}[\mathrm{k}]+\mu^{+}\left(\Delta P[k]-\sigma \Delta W^{\max }[k]\right)^{2} \\
+\mu^{-}\left(. \sigma \Delta W^{\min }[k]-\Delta P[k]\right)^{2}
\end{gathered}
$$

where $\mu$ is the penalty factor.

These relaxations affect feasibility and price elasticity of the DER-level problem. This is affected by several factors such as the type of DER, the present operating conditions, comfort requirements and the prices to which the DER responds to. The wider the band, the greater the elasticity of the bid for the base formulation. All factors directly or indirectly affect the width of these bands. 


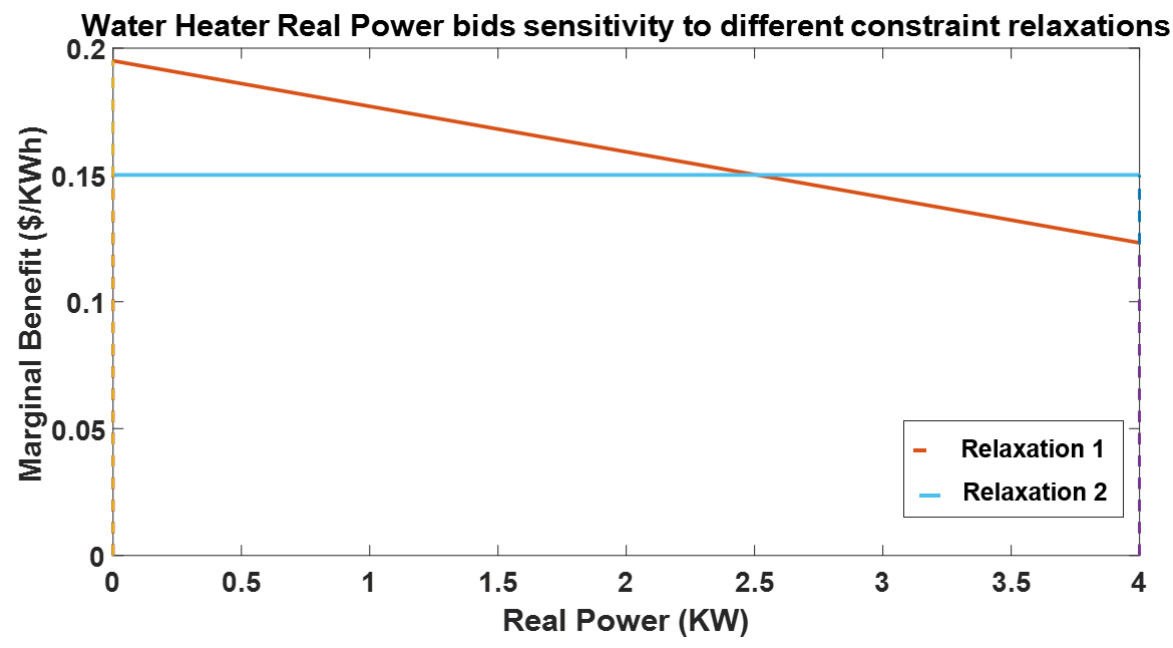

Figure MIT-6(a) Bid sensitivity of WHs to various relaxations

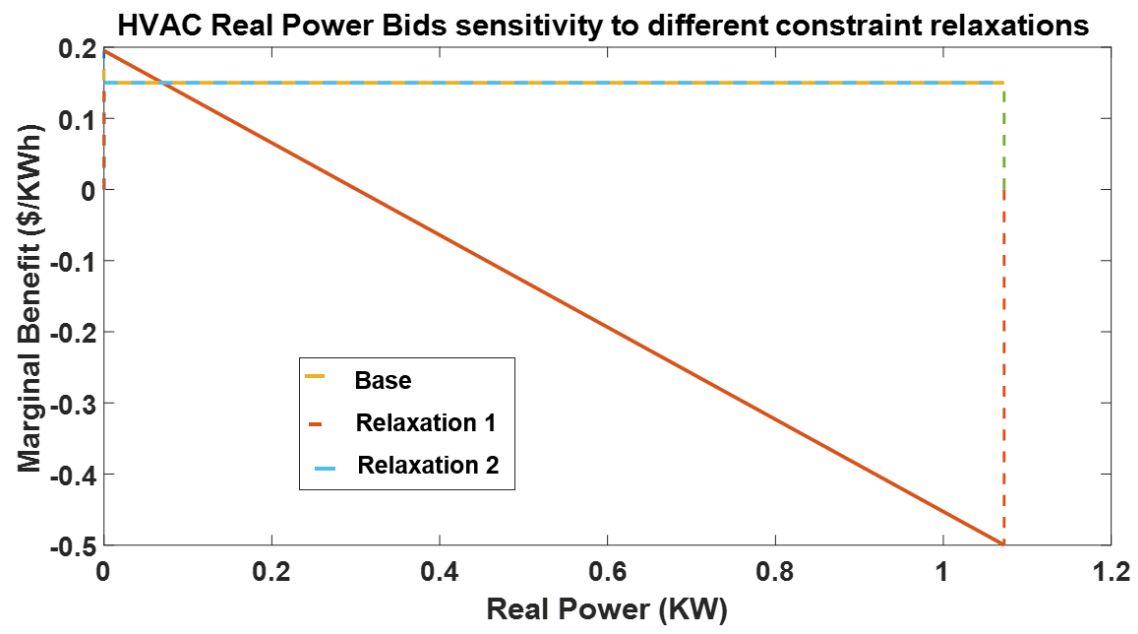

Figure MIT-6(b) Bid sensitivity of HVACs to various relaxations

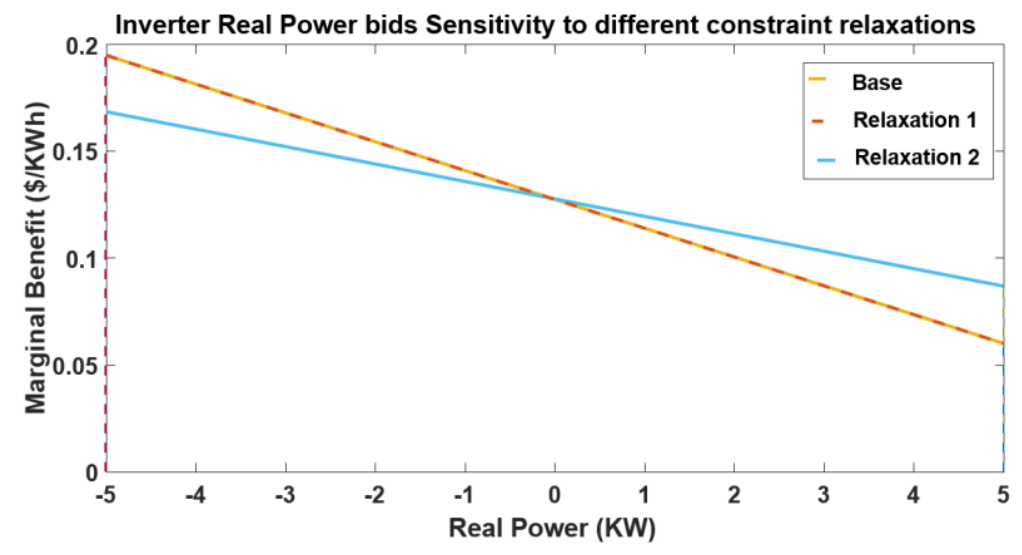

Figure MIT-6(c) Bid sensitivity of inverters to various relaxations

Shown in Figure MIT-6 are typical bids of WHs, HVACs and inverters for when the comfort constraint is considered through two different relaxations. The WH bid computation problem is infeasible for the base case and relaxation 1 yields some price elasticity. For HVAC, all cases are 
feasible, but due to its steady comfort requirements, it is not price elastic in the base case or relaxation 2. Finally, the inverter bids are elastic for all the variations and is seen to be more elastic for the base case and relaxation 1 compared to relaxation 2 . This however may change as the state of charge of the battery approaches its limits.

\subsubsection{Comparison of economic metrics}

We consider economic metrics using two types of utility generation costs; the scenarios will be referred to as 'Distribution tariff' and 'LMP-based tariff'.

Distribution tariff: Given the NIST TE Challenge prices for Case 3, i.e. for TOU pricing as 0.11 $\$ / \mathrm{kWh}$ for most times of the day, except during the solar dip between $15^{\text {th }}$ and $19^{\text {th }}$ hours when the price shoots up to $0.15 \$ / \mathrm{kWh}$, we assume its time average of $0.117 \$ / \mathrm{kWh}$, for analysis of Cases 1 and 2, denoted as uniform pricing in Figure MIT-7. For NIST TE Case 4 however, we utilize the cleared nodal LMPs. These values are time-varying and depend on the present grid supply demand scarcity and are also dependent on the assumed generation cost of the utility generator. It must further be noted that the utility generation price depends on the fuel mix of the generators used upstream of the distribution feeder. We assume here that this cost is constant and is equal to $0.15 \$ / \mathrm{kWh}$. This assumption on utility generation cost results in an LMP value that varies only in the initial times and settles at later times as shown in purple in Figure MIT-8. This occurs because only a small number of DERs were assumed to be controllable and submitting bids. So, utility generation was required to serve the rest of uncontrolled DERs at most times. We then utilize these quantities as the variable component of the tariff that will be used to compute the variable component of revenue and bills for comparison in Table MIT-4.

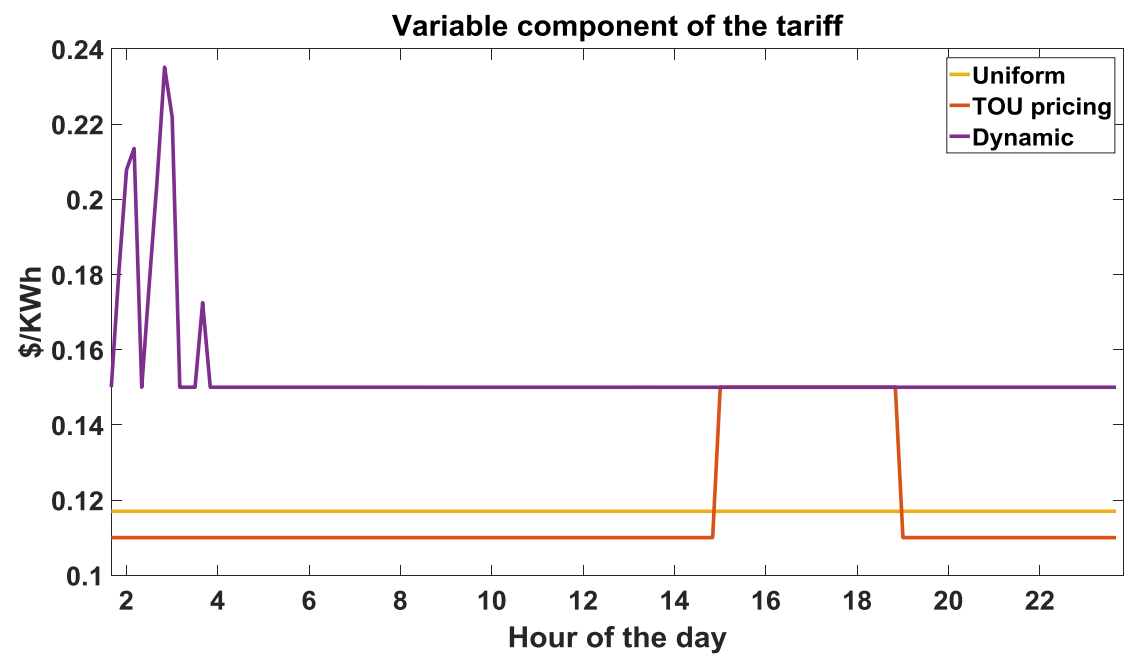

Figure MIT-7 Variable component of the tariff in tariff-driven pricing

LMP-based tariff: In this approach, we recognize that the utility generation costs used in the pricing mechanism above are unreasonable because in general this cost must keep changing over time and the cost of $0.15 \$ / \mathrm{kWh}$ is not appropriate. We thus utilize typical time-varying LMP values of the substation in Arizona on a typical summer day [27]. These time-varying LMPs are utilized to then set a component of the tariff used for computation in NIST TE Cases 1 and 2. This uniform price is shown in yellow in Figure MIT-8. The variable component of the tariff for assessment of Case 3 can be designed by utilizing two different TOU rate structures computed 
by taking the time average of LMPs at the utility bus at different times of the day. Finally, Case 4 analysis is done using instantaneous time-varying LMPs, which is a result of the utility generation cost assumed and is shown in purple in Figure MIT-8.

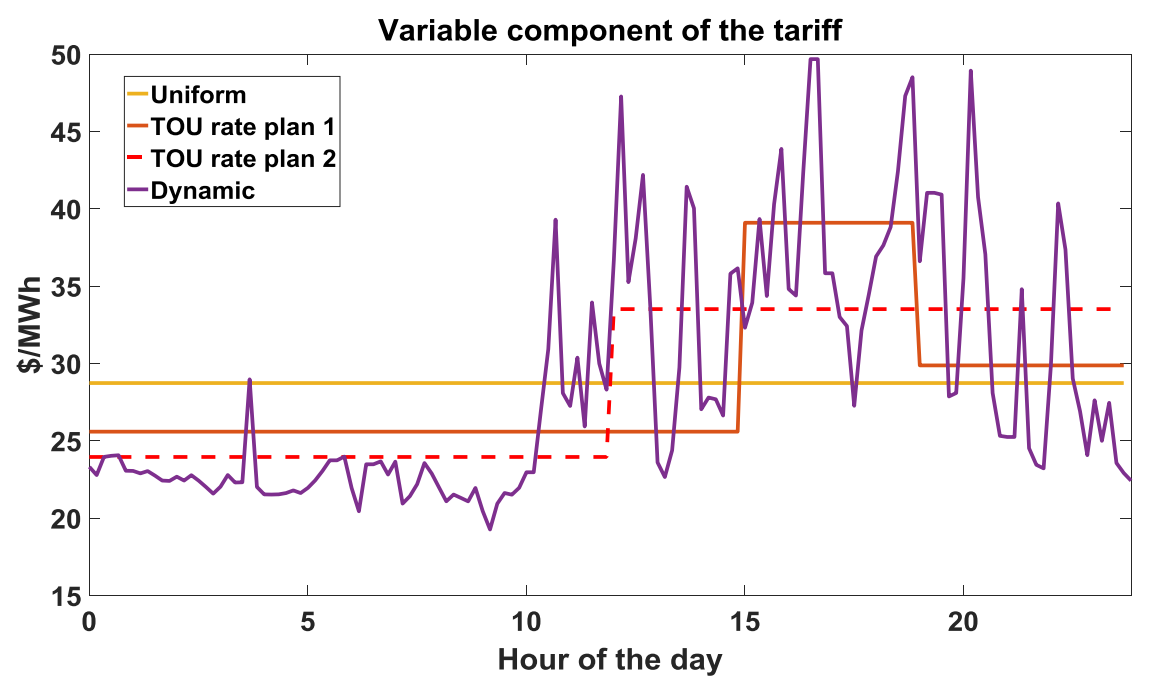

Figure MIT-8 Possible tariff structures

We have considered the LMP-based and Distribution tariff economic metrics to see how the NIST TE cases differ with the tariff structure. However, it must be noted that none of these maps to the actual costs incurred. This analysis offers only a relative comparison based only on the variable component of the tariff. This helps us analyze the relative effectiveness of TEC cases from an economic standpoint.

\subsubsection{DERs as price makers}

For very large utility generation/consumption, the effect of DERs was minimal. In such a case, the results obtained through TOU pricing were similar to those of dynamic pricing. This is because both TE Cases 3 and 4 submit the time-varying limits of real and reactive power consumption to its coordinator. The infinite utility generation always leads to it being marginal unit and thus the cleared prices are not affected by the little DERs in TE Case 4, resulting in identical schedules and cleared prices in TE Cases 3 and 4. Hence, utility generation was restricted to follow a certain policy that its generation would be just sufficient to meet a little over the plug load requirement alone. This policy resulted in considerable changes in energy consumption patterns and revenues in Cases 3 and 4. We will show through numerical analysis how these affect the market outcomes in the following section. We then perform static and continuous time analysis of this system. The purpose of performing static analysis is to understand the inter-dependence of power injections (plug load and DERs) and resulting grid voltages. Next, the continuous time analysis is done to assess the value of predictions brought about by utilizing MPC-based controllers.

\subsubsection{Static analysis}

We consider three representative time snapshots and perform the analysis for different levels of inverter control of PVs and batteries. HVACs and WHs are treated in this static analysis as uncontrollable loads. The analysis results are shown in Tables MIT-2 to MIT-4. The modeling of inverters utilized for grid optimization is identified by a number as follows: 
1. Solar PVs injecting uncontrollable real power equal to the power corresponding to the solar radiation and zero reactive power into the grid.

2. Solar PVs injecting uncontrollable real power equal to the power corresponding to the solar radiation and controllable reactive power (positive or negative) into the grid.

3. Solar PVs with the aid of battery storage, injecting controllable real and reactive powers (both positive and negative) into the grid.

It should be noted that PV Model 1 closely represents method of operation of solar PV installations today. PV Model 2 corresponds to the method of operation of solar PV for large installations. Finally, Model 3 is the method of control envisioned with PV installations supplemented with battery storage. Note that this model does not account for state of charge of the battery and it is assumed that the battery has a capability of injecting or absorbing the rated capacity of real power at any instant of time. Model 3 is further studied for three different scenarios: Model 3.1 represents control of combined PV-battery so that the grid loss is minimized; Model 3.2.1 represents control of combined PV-battery so that the economic dispatch is performed, with the cost functions representing TOU tariffs; and Model 3.2.2 is the economic dispatch minimization with the cost functions being LMP at bus 1 given in [27] at the respective time instant, with PV-battery cost functions for analysis studied using Models 3.2.1 and 3.2.2 assumed to be zero.

The critical time instances considered are as follows:

1. Time $=10 \mathrm{~h}$ : Close to nominal loading profile and high solar PV injections resulting in negative net substation load

2. Time $=15 \mathrm{~h}$ : Close to nominal load profile and sudden dip in solar PV injections

3. Time $=20 \mathrm{~h}$ : Peak load with approximately zero solar PV injections

We consider PV Models 1, 2 and 3.1 in Table MIT-2. Here, the grid loss minimization is the sole objective, while satisfying grid nodal and flow constraints. We further analyze scenario 3.1 to see how PV-battery participation in market transactions can affect different grid entities from both technical and economic perspective.

In scenario 3.2, the incentives are the tariffs that drive the decision-making of individual DERs. For having the same order magnitude, we perform system-level optimization by considering that the utility generation cost is $150 \$ / \mathrm{MWh}$ representing the typical distribution utility tariff of 0.15 $\$ / \mathrm{kWh}$ specified as part of TE challenge data. We further differentiate the cases of unconstrained utility generation in scenario 3.2.1 and constrained utility generation in 3.2.2.

The results obtained for all of these scenarios are tabulated in Tables MIT-2 to MIT-4. Observe in Table MIT-4 that the fixed real power load in scenario 1 is different from that in other scenarios since the solar PVs are being treated as negative loads without any control, thus resulting in lower net load seen by the substation. 
Table MIT-2 Power generation/ Consumption metrics

\begin{tabular}{|c|c|c|c|c|c|c|c|c|c|c|}
\hline $\begin{array}{l}\text { Time } \\
\text { Instant }\end{array}$ & Scenario & $\begin{array}{c}\text { Total Real } \\
\text { Power } \\
\text { Generation } \\
(\mathrm{kW})\end{array}$ & $\begin{array}{c}\text { Total } \\
\text { Reactive } \\
\text { Power } \\
\text { Generation } \\
\text { (kVAR) }\end{array}$ & $\begin{array}{l}\text { Total Fixed } \\
\text { Real Power } \\
\text { Load (kW) }\end{array}$ & $\begin{array}{l}\text { Total Fixed } \\
\text { Reactive } \\
\text { Power Load } \\
\text { (kVAR) }\end{array}$ & $\begin{array}{c}\text { Utility } \\
\text { Real } \\
\text { Power } \\
\text { Generation } \\
(\mathrm{kW})\end{array}$ & $\begin{array}{c}\text { Utility } \\
\text { Reactive } \\
\text { Power } \\
\text { Generation } \\
\text { (kVAR) }\end{array}$ & $\begin{array}{c}\text { Real } \\
\text { Power } \\
\text { Losses } \\
(\mathrm{kW})\end{array}$ & $\begin{array}{c}\text { Reactive } \\
\text { Power } \\
\text { Losses } \\
\text { (kVAR) }\end{array}$ & $\begin{array}{c}\text { Shunt } \\
\text { Power } \\
\text { Injections } \\
\text { (kVAR) }\end{array}$ \\
\hline \multirow[t]{5}{*}{$10: 00$} & 1 & -1154.2 & -118 & -1156 & 271 & -1154 & -118.3 & 0.308 & -19.9 & -371.5 \\
\hline & 2 & 4609.8 & 299 & 4613 & 271 & -839.8 & 161.6 & 1.7 & -23 & 48.8 \\
\hline & 3.1 & 4604.6 & 367.6 & 4613 & 271 & -48 & 330 & 0.08 & -25.5 & 123.8 \\
\hline & 3.2 .1 & 4974.8 & 561.4 & 4613 & 271 & 10525.5 & -521.4 & 368.95 & 683.2 & -413.4 \\
\hline & 3.2 .2 & 4603.4 & 319.7 & 4613 & 271 & -941.1 & 733.4 & 5.62 & -14.45 & 59.78 \\
\hline \multirow[t]{5}{*}{$15: 00$} & 1 & 3527 & -85 & 3503 & 95 & 3527 & -86 & 28 & 28 & -214 \\
\hline & 2 & 4607 & 5 & 4585 & 95 & 3560 & -81 & 28.9 & 28.4 & -122.3 \\
\hline & 3.1 & 4581.9 & 88.7 & 4585 & 95 & 200.6 & 93 & 0.76 & -25 & 18.1 \\
\hline & 3.2 .1 & 4926.4 & 279.3 & 4585 & 95 & 10410.8 & 692.6 & 345.93 & 638.3 & 279.31 \\
\hline & 3.2 .2 & 4591.9 & -1311.6 & 4585 & 95 & 1371.6 & -788.8 & 110.6 & -5.8 & -1401.3 \\
\hline \multirow[t]{5}{*}{$20: 00$} & 1 & 3535.2 & -623.4 & 3511 & 103.3 & 3535 & -623 & 29 & 30 & -161 \\
\hline & 2 & 5094.7 & 419 & 5076 & 103.3 & 3564.7 & 238.06 & 29.3 & 28.8 & 284 \\
\hline & 3.1 & 5063 & -114.6 & 5076 & 103.3 & 204.8 & 152.7 & 0.45 & -102.4 & -184.6 \\
\hline & 3.2 .1 & 5456.9 & 486.7 & 5076 & 103.3 & 11012.2 & -298.43 & 385.5 & 713.3 & -358.4 \\
\hline & 3.2 .2 & 5158.4 & 1615.4 & 5076 & 103.3 & 4930.8 & 2285.1 & 99.04 & 162.1 & 1339.1 \\
\hline
\end{tabular}


Table MIT-3 Voltage Profile Metrics

\begin{tabular}{|c|c|c|c|c|c|c|c|c|c|c|}
\hline $\begin{array}{l}\text { Time } \\
\text { instant }\end{array}$ & Case & $\begin{array}{l}\text { Utility } \\
\text { Voltage } \\
\text { (p.u.) }\end{array}$ & $\begin{array}{c}\text { Voltage at } \\
\text { Cap Bank } \\
1 \text { (p.u.) }\end{array}$ & $\begin{array}{c}\text { Voltage at } \\
\text { Cap Bank } \\
2 \text { (p.u.) }\end{array}$ & $\begin{array}{c}\text { Voltage at } \\
\text { Cap Bank } \\
3 \text { (p.u.) }\end{array}$ & $\begin{array}{c}\text { Voltage at } \\
\text { Cap Bank } \\
4 \text { (p.u.) }\end{array}$ & $\begin{array}{l}\text { Minimum } \\
\text { Voltage } \\
\text { (p.u.) }\end{array}$ & $\begin{array}{c}\text { Bus } \\
\text { Corresponding } \\
\text { to Minimum } \\
\text { Voltage }\end{array}$ & $\begin{array}{l}\text { Maximum } \\
\text { Voltage } \\
\text { (p.u.) }\end{array}$ & $\begin{array}{l}\text { Bus Number } \\
\text { Corresponding } \\
\text { to Maximum } \\
\text { Voltage }\end{array}$ \\
\hline \multirow[t]{5}{*}{$10: 00$} & 1 & 1.0 & 1.0023 & 1.0011 & 1.0025 & 1.0023 & 1.0 & 2348 & 1.0036 & 2081 \\
\hline & 2 & 1.0 & 0.9995 & 0.999 & 1.0013 & 0.9999 & 1.0 & 1 & 1.002 & 2382 \\
\hline & 3.1 & 1.0014 & 1.0012 & 1.0013 & 1.00098 & 1.00314 & 1.0 & 1 & 1.0016 & 646 \\
\hline & 3.2 .1 & 1.0157 & 0.9799 & 1.0029 & 1.0131 & 0.9832 & 0.9692 & 2490 & 1.0157 & 1 \\
\hline & 3.2 .2 & 1.0132 & 1.0117 & 1.0105 & 1.0129 & 1.00934 & 1.009 & 1287 & 1.0133 & 1 \\
\hline \multirow[t]{5}{*}{$15: 00$} & 1 & 1.0 & 0.990 & 0.9976 & 0.999 & 0.994 & 0.988 & 2246 & 1.0 & 2348 \\
\hline & 2 & 1.00518 & 1.00223 & 1.0046 & 0.9950 & 0.9986 & 0.9931 & 2247 & 1.00518 & 1 \\
\hline & 3.1 & 1.0027 & 1.00259 & 1.00265 & 0.9994 & 1.0023 & 0.999 & 2247 & 1.0027 & 1 \\
\hline & 3.2 .1 & 1.0343 & 0.9996 & 1.0224 & 1.0038 & 1.03179 & 0.9919 & 2490 & 1.035 & 1 \\
\hline & 3.2 .2 & 1.0175 & 1.0072 & 1.0133 & 1.0177 & 1.0074 & 1.0047 & 734 & 1.0177 & 2517 \\
\hline \multirow[t]{5}{*}{$20: 00$} & 1 & 1.0 & 0.9897 & 0.997 & 0.9997 & 0.9937 & 0.988 & 576 & 1.0 & 1 \\
\hline & 2 & 1.00461 & 1.001376 & 1.0039 & 0.9944 & 0.99736 & 0.9927 & 576 & 1.00461 & 1 \\
\hline & 3.1 & 1.002 & 1.00097 & 1.0019 & 0.99888 & 0.99994 & 0.9985 & 138 & 1.002 & 1 \\
\hline & 3.2 .1 & 1.0373 & 0.9980 & 1.0231 & 1.0343 & 1.0023 & 0.9878 & 646 & 1.0375 & 1 \\
\hline & 3.2 .2 & 1.0375 & 1.0077 & 1.0236 & 1.0343 & 1.0076 & 1.0001 & 646 & 1.0375 & 1 \\
\hline
\end{tabular}


Table MIT-4 Economic metrics

\begin{tabular}{|c|c|c|c|c|c|c|c|c|c|}
\hline \multirow{2}{*}{$\begin{array}{l}\text { Time } \\
\text { Instant }\end{array}$} & \multirow{2}{*}{$\begin{array}{l}\text { Inverter } \\
\text { Model }\end{array}$} & \multicolumn{4}{|c|}{ LMP-based tariff } & \multicolumn{4}{|c|}{ Distribution tariff } \\
\hline & & $\begin{array}{c}\text { Social } \\
\text { Welfare }(\$)\end{array}$ & $\begin{array}{c}\text { Utility } \\
\text { Generation } \\
\text { Revenue }(\$)\end{array}$ & $\begin{array}{c}\text { PV Solar } \\
\text { Revenue }(\$)\end{array}$ & $\begin{array}{c}\text { Plug Load } \\
\text { Energy Bill } \\
(\$)\end{array}$ & $\begin{array}{c}\text { Social } \\
\text { Welfare }(\$)\end{array}$ & $\begin{array}{c}\text { Utility } \\
\text { Generation } \\
\text { Revenue (\$) }\end{array}$ & $\begin{array}{c}\text { PV Solar } \\
\text { Revenue }(\$)\end{array}$ & $\begin{array}{c}\text { Plug Load } \\
\text { Energy Bill } \\
(\$)\end{array}$ \\
\hline \multirow[t]{5}{*}{$10: 00$} & 1 & 0.0077 & -4.460 & 22.326 & 17.8523 & -7.6617 & -28.85 & 105.76 & 84.5717 \\
\hline & 2 & -1.2283 & -4.460 & 21.090 & 17.8523 & -5.65 & -20.995 & 99.9093 & 84.5717 \\
\hline & 3.1 . & -0.0325 & -0.1858 & 18.0056 & 17.8523 & -0.4740 & -1.20 & 85.2977 & 84.5717 \\
\hline & 3.2 .1 & 1.4002 & 40.732 & -21.4812 & 17.8523 & 76.8030 & 263.1375 & -101.763 & 84.5717 \\
\hline & 3.2 .2 & -0.0372 & -3.6421 & 21.4572 & 17.8523 & -6.45 & -23.5275 & 101.649 & 84.5717 \\
\hline \multirow[t]{5}{*}{$15: 00$} & 1 & 0.1414 & 20.7740 & 6.3730 & 27.0056 & 0.60 & 88.1750 & 27.050 & 114.6250 \\
\hline & 2 & 0.1296 & 20.9684 & 6.1668 & 27.0056 & 1.425 & 89.00 & 27.0500 & 114.6250 \\
\hline & 3.1 & -0.0183 & 1.1815 & 25.8059 & 27.0056 & -0.0775 & 5.0150 & 109.5325 & 114.625 \\
\hline & 3.2 .1 & 2.0108 & 61.3196 & -32.3031 & 27.0056 & 8.535 & 260.27 & -137.11 & 114.625 \\
\hline & 3.2 .2 & 0.0406 & 8.0787 & 18.9676 & 27.0056 & 0.1725 & 34.2900 & 80.5075 & 114.625 \\
\hline \multirow[t]{5}{*}{ 20:00 } & 1 & -0.0642 & 20.8211 & 9.0117 & 29.8971 & 23.3688 & 88.3750 & 28.0500 & 93.0582 \\
\hline & 2 & 0.1107 & 20.9961 & 9.0117 & 29.8971 & 24.1093 & 89.1175 & 28.0500 & 93.0582 \\
\hline & 3.1 & -0.0760 & 1.2063 & 28.6148 & 29.8971 & -59.8882 & 5.1200 & 28.0500 & 93.0582 \\
\hline & 3.2 .1 & 2.2441 & 64.8619 & -32.7207 & 29.8971 & 80.3997 & 275.305 & -101.847 & 93.0582 \\
\hline & 3.2 .2 & 0.4859 & 29.0424 & 1.3406 & 29.8971 & 34.3845 & 123.27 & 4.1727 & 93.0582 \\
\hline
\end{tabular}




\subsubsection{Observations Based on Numerical Findings}

There are a few notable observations from these tables with respect to several of the metrics.

- Real Power Losses:

- Decrease as the level of inverter control increases for scenarios 1 to 3.1 .

- When the objective function changes from loss minimization in scenarios 1, 2, 3.1 to economic cost minimization in 3.2.1 and 3.2.2, the real power losses increase as expected. The losses in scenario 3.2.1 are considerably higher because the batteries charge instead of injecting power into the grid, owing to the cheaper utility generation. However, in scenario 3.2.2, the lower relative bids sent by PVs result in them being scheduled to provide most of the load, thus reducing the real power losses.

- Reactive power losses: A general trend has been noticed that, during the times when the net real power injection from solar PVs and/or batteries is high, the reactive power losses are negative. This may be attributed to the fact that the distributed power generation results in higher voltages downstream, resulting in higher reactive power injections by the shunt line capacitances than the ones absorbed by the line reactances.

- Shunt Power injections: The scenarios when the grid is devoid of local power generation, i.e. when the solar radiation is almost zero at times 15 and 20 in scenarios 1 and 2, or when cheap utility generation makes the batteries in scenario 3.2.1 consume power, the utility has to supply the household loads. Then, power delivery is feasible only when the shunts act as reactors, increasing net reactive power demand, facilitating real power flow while maintaining grid voltages within limits.

- Voltages:

- For the more complex inverter controls in scenarios 1,2, and 3.1, the voltages are less than 1 p.u. This can be seen in Table MIT-3 at all times.

- With the introduction of generation cost functions in scenarios 3.2.1 and 3.2.2, larger voltage excursions, which are still within the limits, are noticed. This is predominant in Case 3.2.1 when the electrical distances between the cheap utility generator and point of consumption, i.e. homes, are much larger. Recall that these houses are connected at respective MV nodes. The actual voltage at terminals of the house can be lower, which will be seen in detail in Section 5.3.

- Economic metrics: The power generation and consumption values obtained in each of these scenarios are utilized to compute the social welfare of the grid and revenues and bills of several entities involved in the transactions. This is done using two types of tariffs identified by the column headers in Table MIT-4. The one identified as 'LMP-based tariff' is computed as the average of the provided utility LMP [27] during two different time periods of the day. The utility generation cost is taken to be equal to the time-varying utility LMP. These are both shown in Figure MIT-8. The one identified by 'Distribution tariff' refers to the NIST TE challenge TOU tariff that was given and is plotted in Figure MIT-7 and utilizes constant generator operating cost of $150 \$ / \mathrm{MWh}$.

- The social welfare computed using these tariffs can be positive or negative. This quantity generally is positive (or negative) when the customers are charged less (or more) than the 
marginal cost of the generation. Hence, while this quantity is to be maximized at the grid optimization to compute the optimum generation values, the assessment of efficiency of tariff structure when performed using this metric should be based on how close its absolute value is to zero. Zero social welfare implies that all the variable components of cost incurred in the generation and transmission is recovered from the energy bills of the customers.

From our analysis, we have the following observations:

- The plug load revenues for all levels of inverter control are constant for a particular time instant. This needs to be served by a combination of utility generator and solar PVs. Hence, as the solar PV revenues increase, the utility generation revenues decrease. At the tenth hour, excess solar PV results in reverse power flows, selling power to the grid, indicated by negative utility generation revenues. Scenario 3.1 schedules, however, are such that the reverse flows are minimized in the interest of the grid by exploiting the battery charging capacity.

- For the static snapshot, the social welfare is proportional to the real power losses. Hence, Scenario 3.1 (PV-battery control for grid loss minimization) in 'LMP-based tariff' always has the lowest absolute value of social welfare, indicating that the tariff structures used here fit well for a regulated environment with more distributed generation availability. For the distribution tariff considered however, scenario 3.2.2 (PV-battery control for economic dispatch, constrained utility generation) is the best possible method of inverter control.

Note that these values do not directly relate to the actual cost of operation of the grid, which can be computed using the cleared LMPs. The motive for reporting on these metrics is to highlight how different tariffs can result in increased profits or losses of grid entities, and how it affects the social welfare as a whole. Such analysis can then be used to compare the tariff structures and/or design a tariff structure that results in maximization of social welfare. This aspect needs to be pursued in more depth for future work.

\subsubsection{Continuous Time 24-hour MPC-based TEM Simulations}

For purposes of modeling and analyzing the effects of MPC-based scheduling, a continuous time analysis is performed over a period of 24 hours. This is done by considering 250 of the total number of 1700+ available solar PVs as distributed agents with an embedded decision making algorithm. The remaining PVs are treated as inflexible. The $12.47 \mathrm{kV}$ MV grid has been considered for grid optimization of power flows. Next, we show the effects of an LMP-driven pricing mechanism in the simulations. The absence of temporal inter-dependencies in TEC Cases 1 and 2 results in similar dispatch schedules as in the static analysis above. However, the TEC Cases 3 and 4 take into consideration the temporal coupling which is essential for TEM. While DERs communicate their tolerable consumption limits in Case 3, they do not affect the economic outcomes of the grid directly. Case 4 with TE market implementation accounts for DER-granularity requirements and makes DER price-makers by allowing for participation in the system level decision making. We thus consider TEC Case 4 variations in great detail and analyze the results.

Due to the restrictions on availability of compute nodes on MIT-LL Supercloud, we have considered only 250 distributed decision makers. However, there is another smaller system with analogous loading profile and operating problem, on which we have analyzed several variations of TEM along with WH and HVAC embedded control. This analysis can be found in [28]. 


\subsubsection{Analysis of Voltage Metrics}

Figure MIT-9 gives the voltage profile for each of the simulated TEC cases. Notice that the utility bus voltage tends to optimally operate at 0.95 p.u., except when there is a considerable utility generation requirement, as seen after the 17th hour when the net load is high and during the times of solar dip at the 15th hour. The PV dip happens at 14:40, indicated by a sudden rise in Case 2 (Storm) voltages seen in blue. The embedded MPC in DERs predicts higher reactive power prices in the future and adjusts consumption a priori to aid the grid in voltage control either by reducing real power consumption or by increasing reactive power injections into the grid. If the utility bus voltage is set at 0.95 p.u., then the number of voltage excursions from 0.95 p.u. dictate the number of regulator and shunt capacitor tap changes that are needed for voltage control at the utility bus.

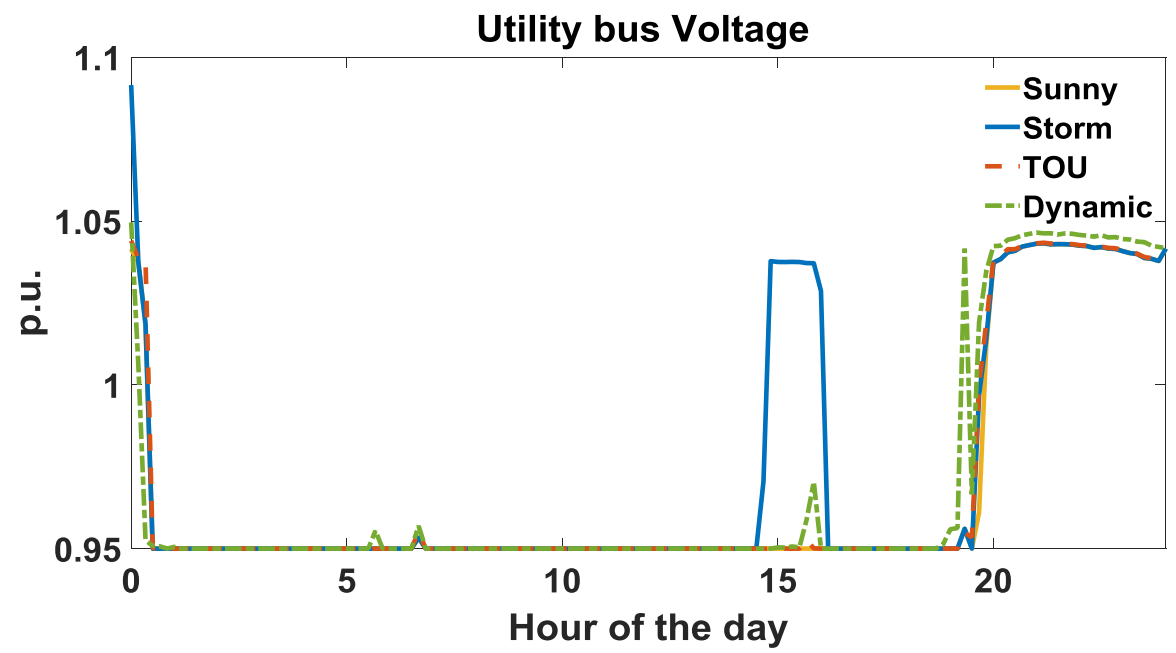

Figure MIT-9 Utility bus voltage magnitude

The maximum and minimum voltages seen in each of the cases are also shown in Fig. MIT-10.

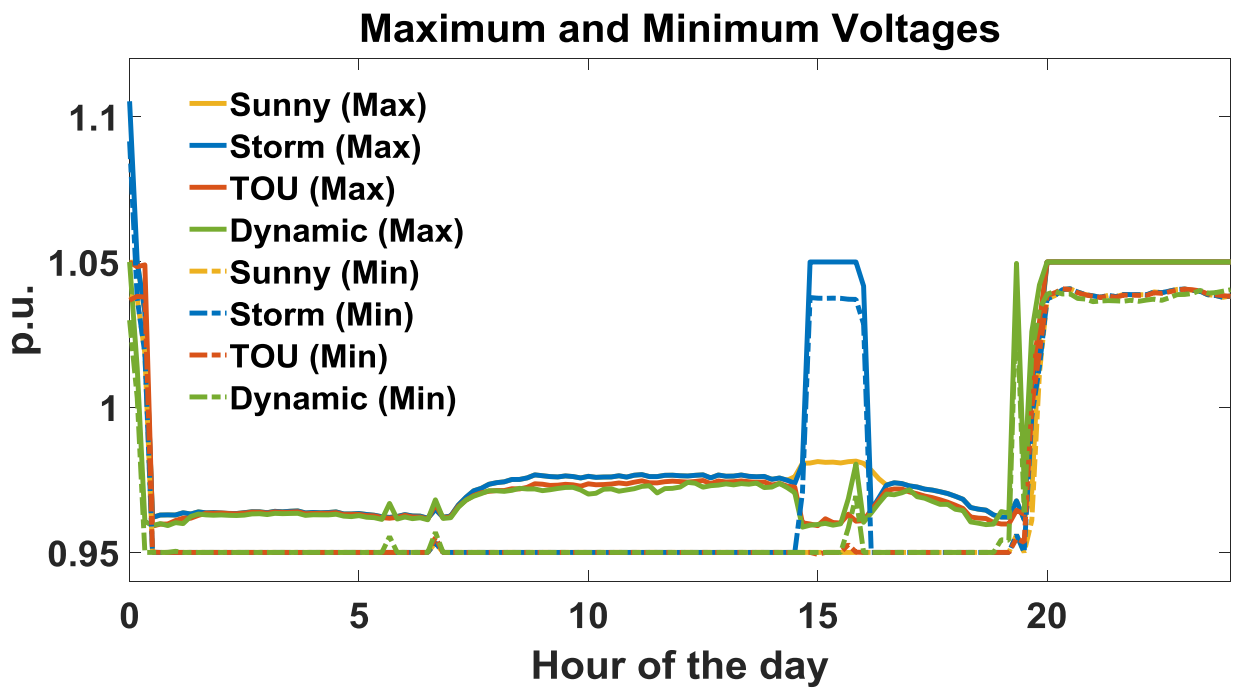

Figure MIT-10 Minimum and maximum voltages seen in the grid 
It can be seen in Figure MIT-10 that the minimum and maximum voltages seen on the MV grid are violated only at time $\mathrm{t}=0$ for excessive loading (ref. Figure MIT-5) in TEC Cases 1-3 (Sunny, Storm, and TOU). This is however circumvented in TEC Case 4 (Dynamic). Notice that the plot corresponding to Case 4 (in green) is always within the chosen voltage limits of 0.95-1.05 p.u. Furthermore, the differences between the minimum and maximum voltage values are larger in Cases 1 and 2. As a comparison, however, the TOU and dynamic pricing cases result in closer minimum and maximum voltage profiles. This may be attributed to the presence of batteries in these cases injecting real and/or reactive power when the grid is in need. The voltage sensitivities seen by the regulating transformer at the utility bus are shown in Figure MIT-11.

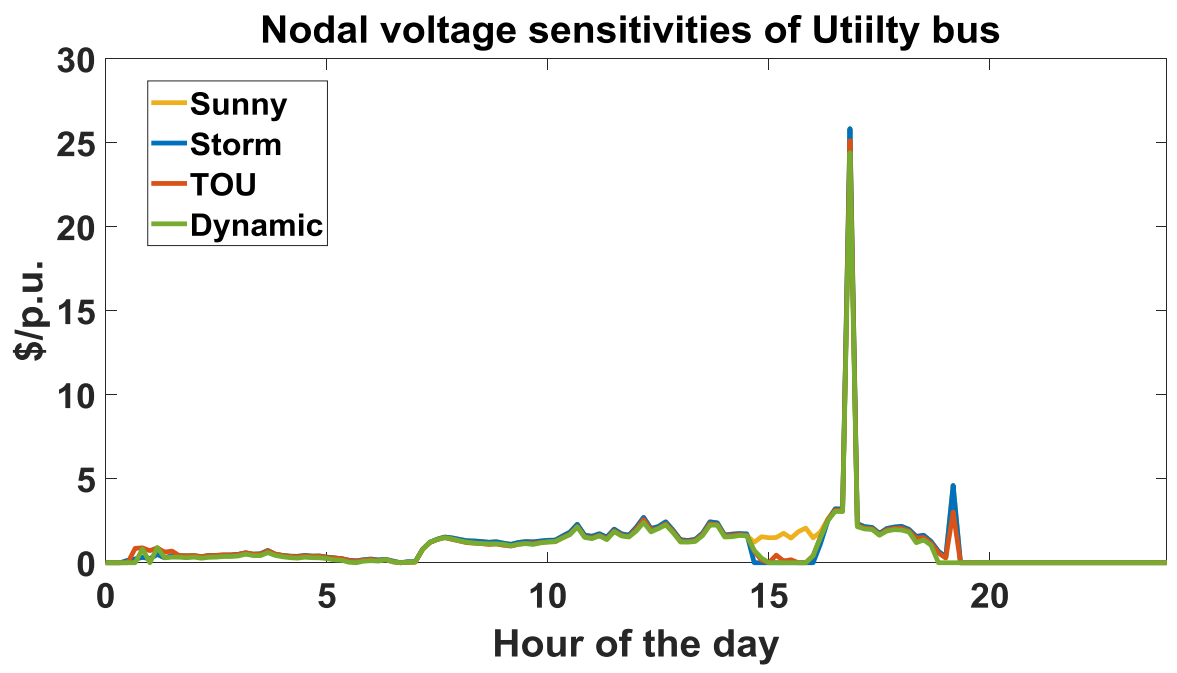

Figure MIT-11 Voltage sensitivities

The voltage sensitivities are non-zero whenever the limits of voltage are reached and can thus act as a measure of cost incurred in maintaining grid voltage. In Case 4, this sensitivity is used as an incentive signal for DERs to adjust their real and reactive powers. There is a small-time delay for case 4 as compared to the other cases which almost overlay each other. This may be due to the effect of MPC delaying voltages in reaching their lower threshold.

The reactive power injections from one of the regulating shunts labeled as node ' $R 18242$ ' in the IEEE 8500 grid node naming is shown in Figure MIT-12. For most time instants this shunt acts as a regulator absorbing excess reactive power for voltage support. It is seen that the number of these capacitors switching tends to be higher in Cases 3 and 4 where there is additional DER-level control. The spikes seen in Case 4 are reactive power injections that may be attributed to the conflicting objectives of DER-level reactive power controllers and grid-side controllers. These spikes are absent in TOU pricing since the price signal does not act as feedback to drive the reactive power injections in Case 3. From these plots, it appears that the terminal voltage control of DERs themselves, adjusting real and reactive power consumptions, may result in short-term voltage violations at the grid nodes which are supposed to be regulated by the DSO. As a result, more control actions might have to be taken by the DSO to minimize the voltage violations at these nodes. Furthermore, there is also some oscillatory behavior in the DER price response to ensure voltage violations do not occur. This is a problem that needs to be looked into more carefully in the future. 


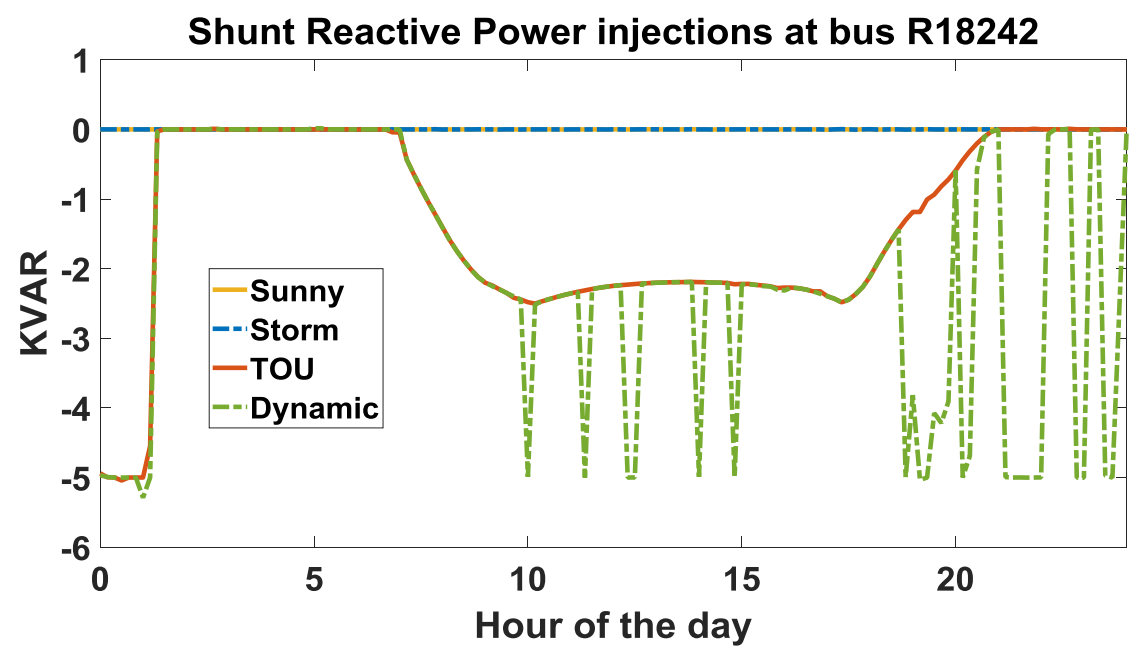

Figure MIT-12 Shunt capacitor reactive power injections at node 'R18242'

\subsubsection{Power Generation/Consumption Metrics}

Shown in Figure MIT-13 and MIT-14 are the real and reactive power injections into the grid. Notice that power is injected into the substation (reverse flows) from 8-15 hours, after which a sharp dip in solar radiation calls for a sudden utility generation requirement. The power generation schedules obtained by TOU and dynamic pricing overlap each other during most time instants since both these cases have additional storage devices. However, the internal reactive power control done by the DERs result in a reactive power injection into the utility that is much less than the ones seen in other cases.

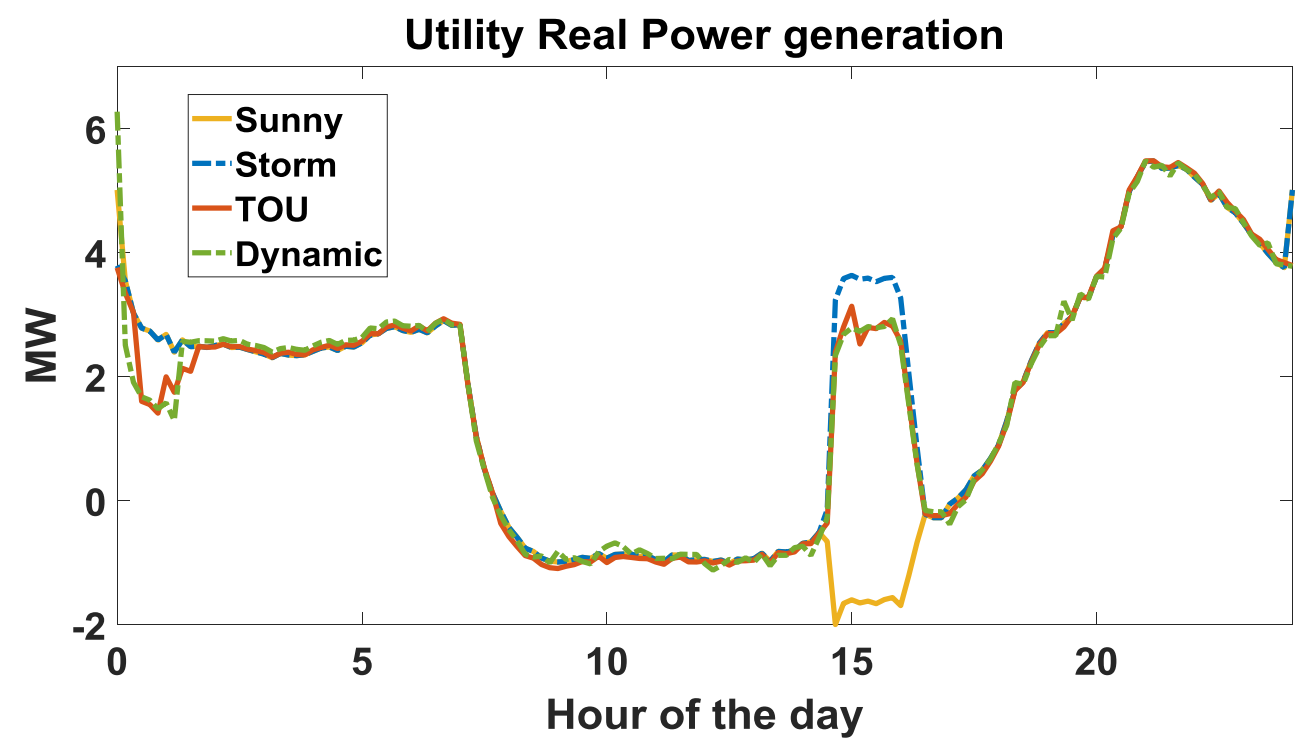

Figure MIT-13 Utility real power generation 


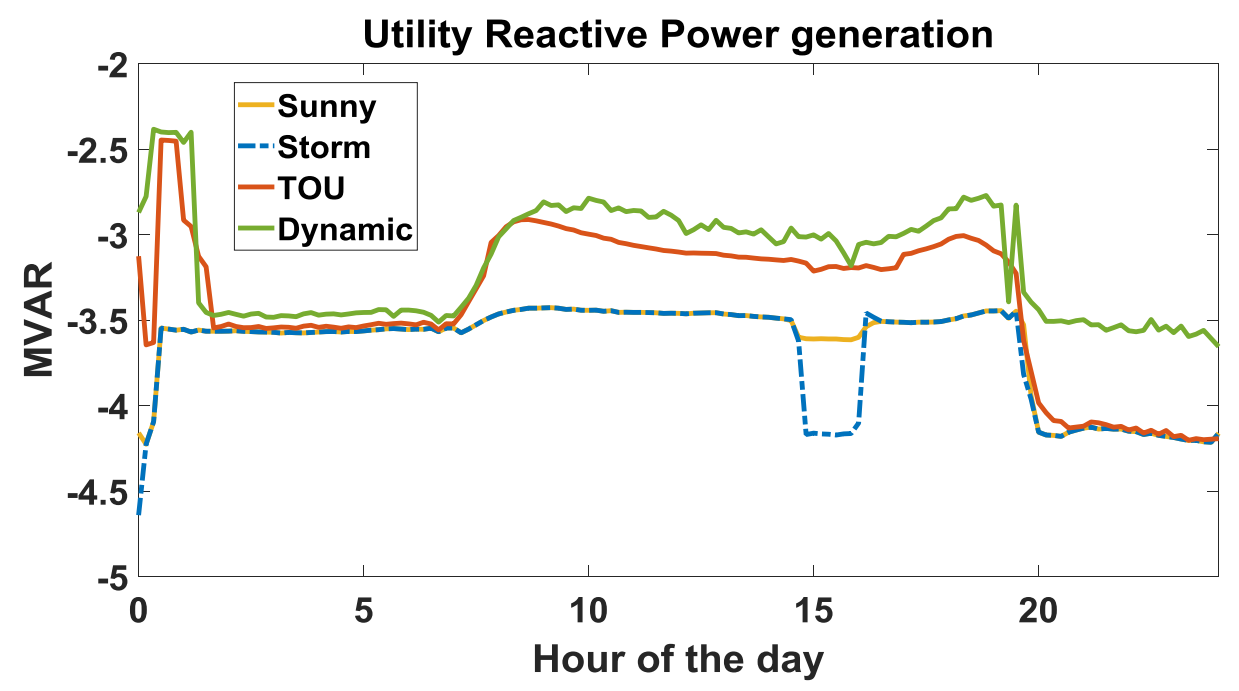

Figure MIT-14 Utility reactive power generation

It can be seen in Figure MIT-15 that the embedded control inside DERs also results in reduced total real power losses, for the same reasons as previously stated for decreased real power requirement from the utility. As the solar penetration decreases during the hours 11-15, the extent to which PVs can participate decreases. As a result, there is an increased need for utility generation which will result in increased grid power losses. The losses, however, are lower in TE Cases 3 and 4 because of the storage devices that could be utilized until the battery is exhausted.

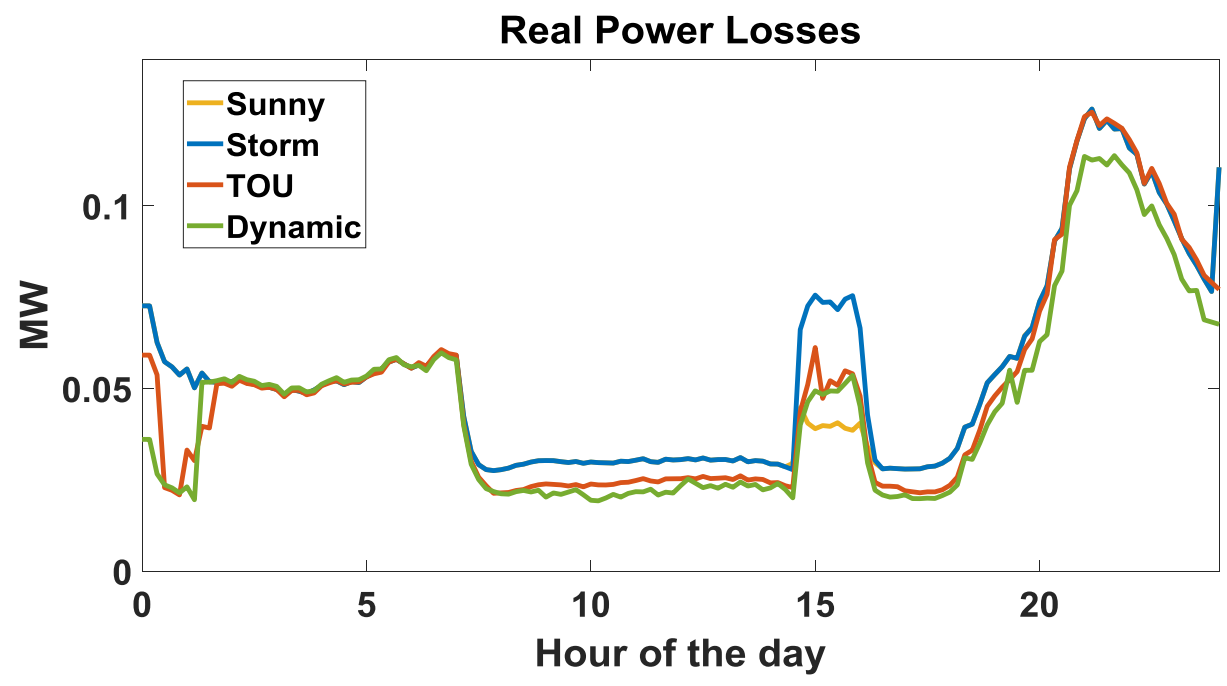

Figure MIT-15 Real power Losses

\subsubsection{Economic Metrics}

The dispatch quantities along with the time-varying LMPs are utilized to compute the operating cost of utility generation. Figure MIT-16 shows that the utility operating costs are lowest in TEC Case 1 since there is excess solar generation calling for reduced real power requirement from the utility during most times of the day. 


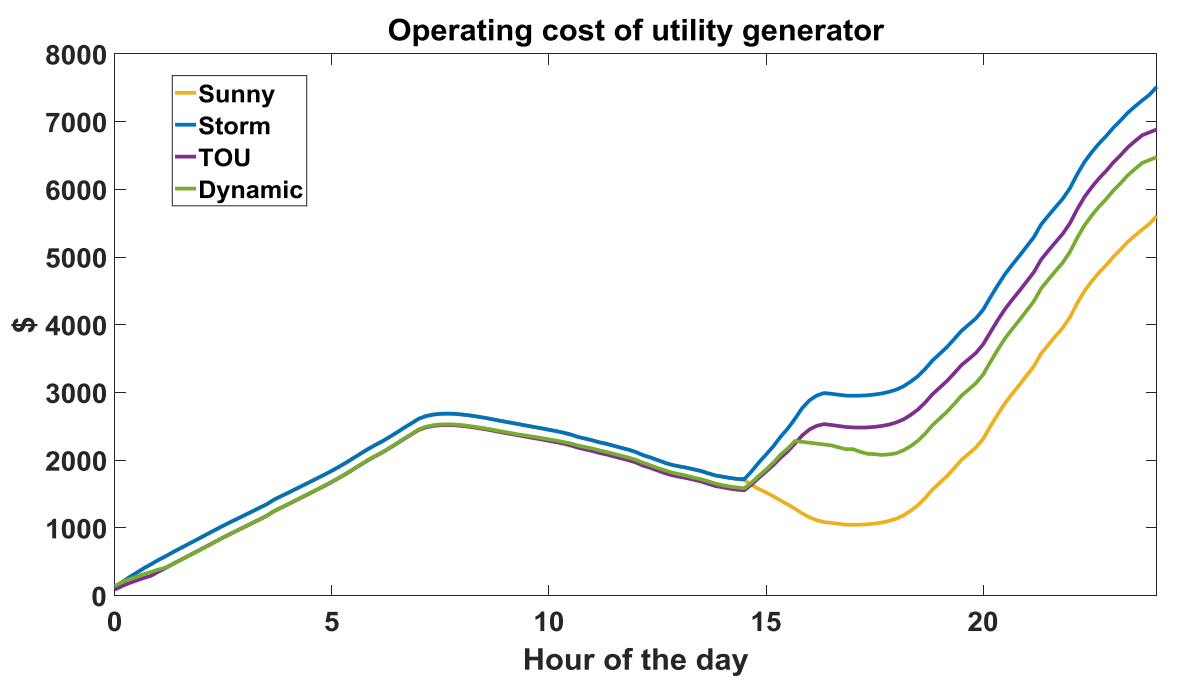

Figure MIT-16 Cumulative operating cost of utility generator

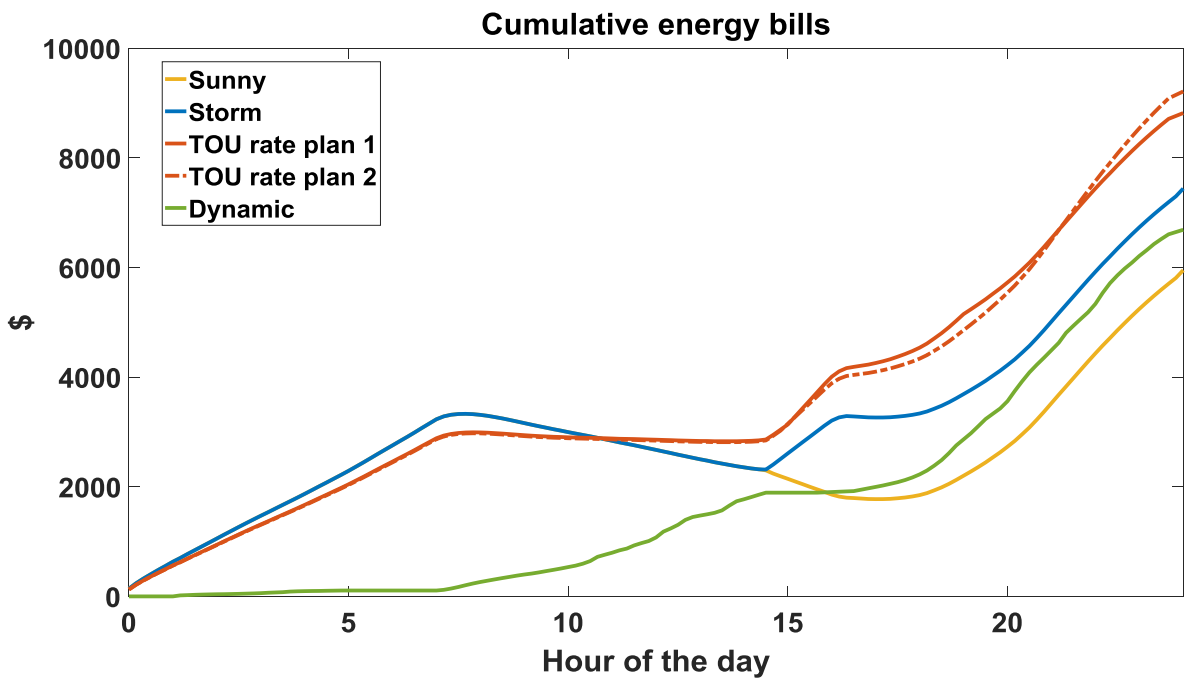

Figure MIT-17 Cumulative energy bills of all the houses

Similarly, the net customer energy bills are computed for different tariff structures as described in Section 5.1 under LMP-based tariffs and are shown in Figure MIT-17. These are compared to the dynamic pricing that utilized instantaneously cleared LMPs. The cumulative energy bills are found to be cheapest in TEC Case 1, again due to excess solar PV generation. Out of the rest of the cases with the storm front considered, the dynamic pricing strategy results in the lowest cumulative energy bills. Both of the TOU rate plans for the large system are overcharging customers.

\subsubsection{Grid Analysis}

So far, we have done the analysis of the IEEE 8500 system by assuming all the houses are incident directly on the MV node. However, there exists a network that connects these houses to the grid. A typical connection of the MV node to the houses through center tapped transformers and triples wires is shown in Figure MIT-18. 


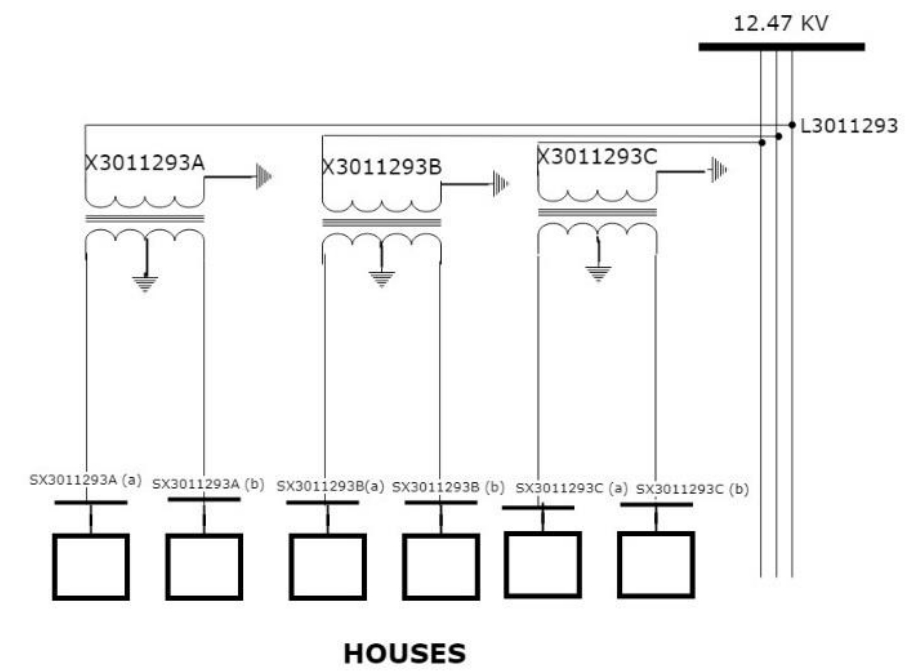

Figure MIT-18 Typical US distribution sub-system

Loading profiles of houses on each of the phases are shown in Figure MIT-19. We will now utilize this loading pattern and the voltage at the MV node as found in TE Cases 2-4 to examine if the voltages at house nodes identified by node names starting with 'SX' in Fig. MIT-19 violate ANSI standards.
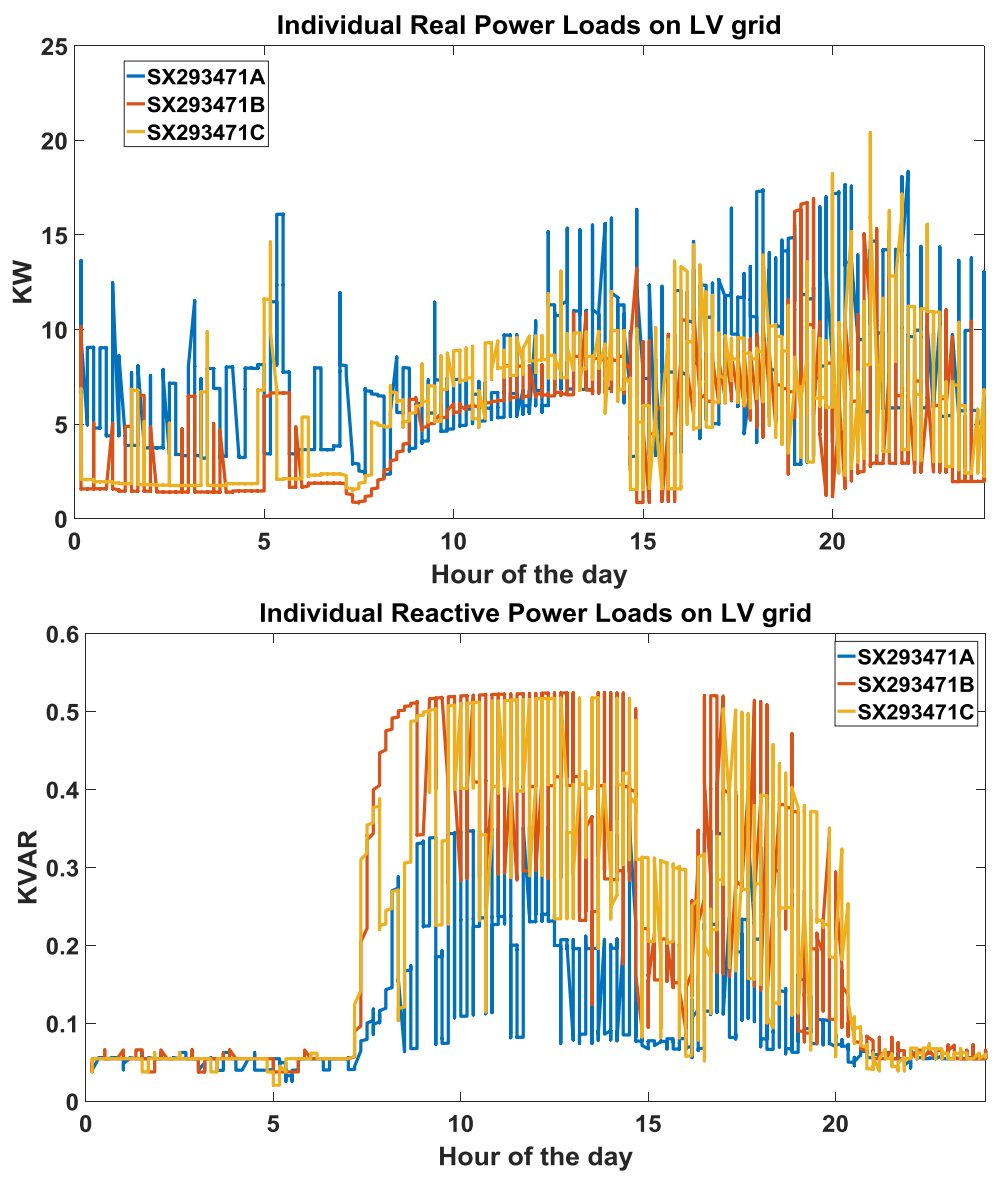

Figure MIT-19 Loading of children LV nodes coordinated by a single MV node 
For nominal utilization voltage, the voltage drops across different phases, assuming equal loading of the secondary of the center-tapped transformer, is shown in Figure MIT-20. This then corresponds to the minimum voltage limit imposed on the MV node as shown in Figure MIT-21. We can see that at most times of the day, the minimum voltage limit is about 0.96-0.98 p.u., which can be set in the grid optimization software, to ensure ANSI standards at terminals of homes are not violated.

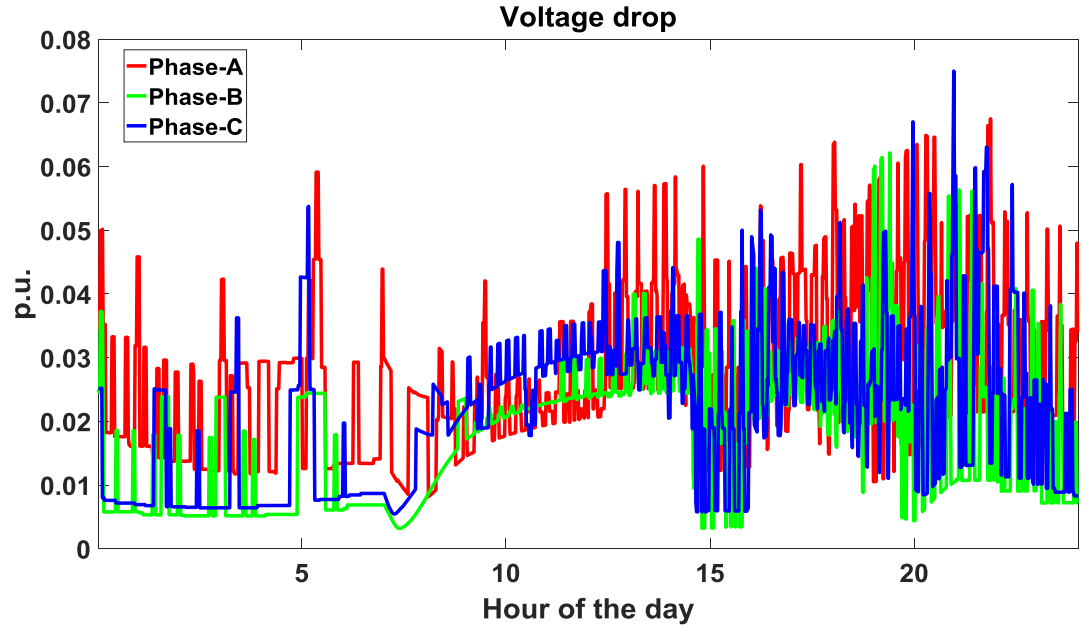

Figure MIT-20 Voltage drop across the service lines and transformers of the secondary distribution system

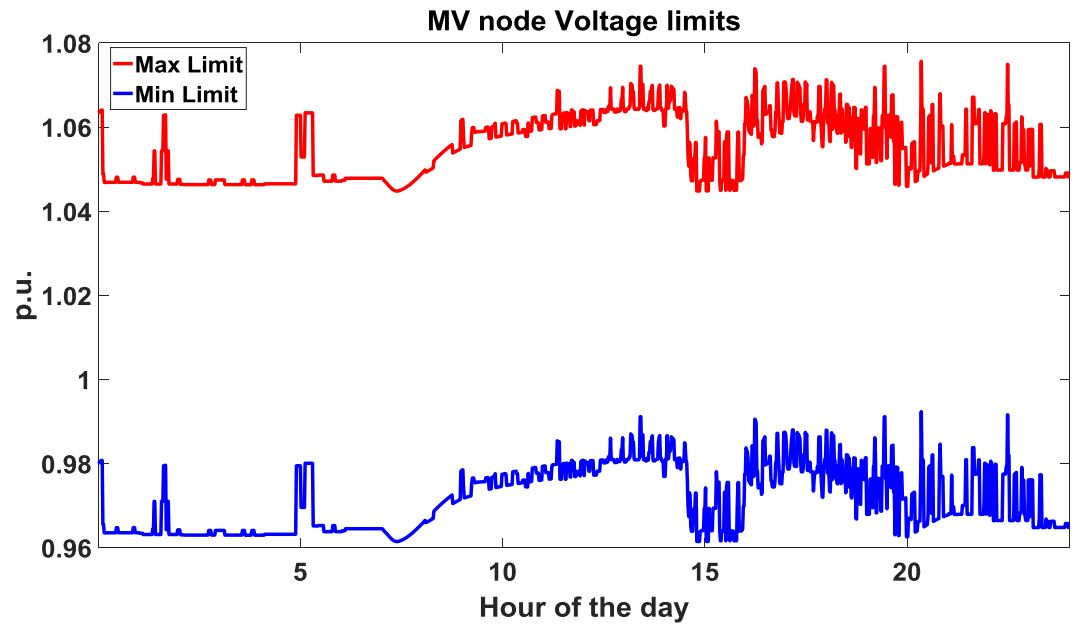

Figure MIT-21 Constraints on voltages of MV nodes

\subsection{Future Utility Role in Transactive Energy Markets}

As a general observation, significant integration of DERs, including solar PVs, critically hinges on how operationally feasible their integration is. Except for the highly isolated stand-alone households equipped with very small storage targeted to serving their own plug-load, distribution grids and microgrids will be essential for delivering power provided by the DERs to the plug-load locations. The IEEE 8500 bus distribution grid is representative of a typical distribution grid for deploying 
efficient and clean power. However, at present the role of the distribution grid is not well-defined and the incentives for supporting large integration of DERs may not be explicit.

Our simulation analyses and economic and technical metrics results can be used as the basis for further discussion of some key open questions and for proposing possible ways forward. Moving forward requires considerable depth in assessing the issues and proposing protocols and standards that may create an environment in which different stakeholders are paid and pay according to the right incentives and tariffs. The resulting metrics support the expected conclusions that both technical and economic performance depend on the technical functions expected by different stakeholders, as well as on the valuation of these functions. For example, voltages must be maintained within the operating limits so that the electricity service to the small end users meets ANSI C84.1 standard. These limits can be observed and maintained in many non-unique ways, such as by solar PVs participating in grid-level voltage regulation, or by the distribution grid regulating its controllable hardware, such as transformer and shunt capacitor/transformer equipment. Ultimately, as shown in this report, the voltage support can be provided in a very effective way by the regulation of substation voltage.

An important technical detail is that, depending on the functionalities/sub-objectives of different stakeholders, (1) the voltage profile can be assessed by power flow analysis to maintain voltage within limits and, if not, local PVs would have feedback control to respond to deviations away from the acceptable ranges; or, (2) more traditionally, the grid operator sub-objective could be to minimize delivery losses, without considering the need to differentiate between utility and DER power delivery.

In this paper a third alternative is considered in which voltage is modeled as a decision variable, along with the real power, to support maximizing social welfare, for example. This is done by using an extended AC OPF capable of optimizing both voltage and real power dispatched/consumed. As the basic observation, one can see from the simulations presented that when net load increases, the power flow analysis simply shows that the resulting voltages decrease. However, when AC OPF is used to support delivery of increased system load the voltages generally increase as a result of adjusting controllable voltage set points elsewhere, for example set points of controllable grid equipment or the voltage set points of controllable solar PVs. This optimization then makes room for delivering more net system load without violating ANSI standards required by the end users. Significant benefits from such voltage optimization are reported.

Establishing incentives to the right stakeholders for supporting power delivery according to ANSI C84.1 standard is quite complex. These incentives can be rolled into typical two-part tariffs comprising fixed charges and pass-through variable cost, such as fuel cost, in particular. The problem with fixed charges at guaranteed rate-of-return is that they are strictly volumetric and do not differentiate between the equipment being controllable or not controllable. This volumetric (capacity-based) approach to tariffs is one of the key causes of utilities' concerns with DERs creating competition to their service. It is shown in this report that the issues regarding energy bills become convoluted in the TEM environment depending on whether fixed two-part, tariffs TOU tariffs (again based on two-part approach or averaged variable cost approach); or entirely LMPbased tariffs are used to give incentives to the DER market participants. In this report we provide ample evidence of how this choice affects all economic metrics. 


\subsection{Preliminary Conclusions: Performance-based Grid Regulation based on TEM Peak Load Pricing}

TEM is modeled and simulated by utilizing our general DyMonDS framework and its supporting computer platform SEPSS. This home-grown simulation platform simulates interactions of DER models with the market clearing compute node, which utilizes third party AC OPF to perform system level optimization. DERs send their bids, and the compute node computes and sends LMPs and optimal schedules back to the DERs. This process was simulated using the IEEE 8500 bus distribution system on the MIT supercomputer, and it was shown for the first time how to implement TEM by dynamically exchanging LMPs while, at the same time, controlling voltages to meet the ANSI C84.1 standard. For this, DERs and their automated control are modeled as generalized energy droops which model sensitivities of real and reactive output power with respect to real and reactive input power. Each DER performs local optimization specific to its own subobjective subject to a power droop constraint to ensure feasible implementation; the result is a bid function that defines price for producing/consuming real and reactive power. The DER bids are communicated to the SEPSS compute node which then interacts with NETSSWorks, which uses ACOPF to clear bids so that system performance metrics of interest are optimized. The results of this optimization, both LMPs and optimal schedules, are sent to DERs which use it for future bidding. Using ACOPF is the key to optimizing voltages, further ensuring that real and reactive power are balanced, subject to voltage and grid constraints. The voltage constraints at the MV $(12.47 \mathrm{kV})$ level were maintained between 0.95 p.u. and 1.05 p.u in order to meet ANSI C84.1 standards at the LV level.

TEC Cases 1 and 2 have only system-level performance metrics defined. To study these cases, an algorithm for system level decision making has been embedded on a single compute node, which is responsible for emulating the coordination activities by interacting with a load forecaster module that sends the anticipated net consumption at each of the nodes in the grid. This compute node then interacts with NETSSWorks to optimize system performance. Voltage control in Cases 1 and 2 only involved controllable shunt regulators and capacitors. It is shown that when there is net negative load because of large solar PV installations at homes, it becomes necessary to dump extra power. Alternatively, grid protection and control could be designed to send excess power to the rest of the grid. Our simulations show that MPC-enabled storage control gets around this problem at least partially.

With regards to economic performance, we have computed the resulting social welfare for three representative loading levels. For comparison across all four cases, these metrics were computed using two approaches:

(1) LMP-based tariff: time-varying values of LMP at a substation bus in Arizona on a typical summer day was assumed to be the generation cost of the utility generator. The TEC Cases 1 and 2 utilized the time-average of this LMP over the entire day. TEC Case 3 computed the time-average for different times of the day to resemble the TOU price. Finally, TEC Case 4 utilized the actual cleared LMPs; and,

(2) Distribution tariff: The TE Challenge-provided TOU electric distribution charges were utilized for TEC Case 3. The time average of this TOU tariff is used as the flat-price tariff for TEC Cases 1 and 2. Finally TEC Case 4 utilized cleared LMPs where the utility generation cost was assumed to be equal to the peak value of the TOU distribution tariff. 
The LMP-based tariff and distribution tariff differed by orders of magnitude. All the economic metrics were computed for both of these tariff structures for representative load levels.

To assess performance of TEC Case 3, a scenario was simulated with device-specific algorithms on additional computing cores to emulate distributed decision makers and their communications with the utility node. This is the same as that for TEC Cases 1 and 2, except that estimations of plug loads in TEC Cases 1 and 2, along with controllable demand specifications, are obtained before the utility node clears the bids through NETSSWorks ACOPF. In Case 3, the agents are price-takers, performing decision making based on anticipated TOU prices to identify the bounds on consumption for the next market interval. In doing so, no price information gets communicated to the utility node. Due to the MPC-based decision making, the grid voltage profile is changing less frequently, requiring fewer regulator tap-changing actions, and all together avoiding excessive wear and tear. Also, it has been observed that the reactive power consumed by utility generator (needed to compensate capacitive reactance of wires) is reduced significantly.

Finally, TEC Case 4 follows the same setting as Case 3, except that the distributed decision makers, in addition to time-varying limits on real and reactive power, also submit the price bids which are quadratic functions expressed as a function of power. This ultimately creates a win-win situation where the social welfare is maximized through distributed agents which become price-makers. With regards to technical performance metrics in TEC Case 4, it is important to note that agents respond to cost sensitivities with respect to both real power and voltage proximity to their limits. The bids and market incentives are only provided every ten minutes (the energy market clock) for the worstcase anticipated voltage violations and for the anticipated real power consumption. Notably, using our recently introduced notion of a quasi-static technology-specific droop, one ensures that within 10 minutes no voltage violations occur. This is the responsibility of the agents themselves. This makes the MIT approach qualitatively different from the other teams' approaches which do not utilize the physically implementable device characteristics (droops) when bidding. Because of this, their approaches require much faster communication, and/or they need to implement feedback control to avoid voltage violations. The effect on all performance metrics when compared with TEC Case 3 are minimized.

The main lessons learned are that often incentives are not strong enough to enable DER participation. This is particularly the case when there is sufficient utility generation to supply plug load. However, this can also be attributed to numerical issues resulting in locally optimal voltages. It is well-known that ACOPF has flat global optimum that cannot easily differentiate combinations of bids. Given that DER-specific decision variables have orders of magnitude smaller limits when compared to those of the utility generation, numerical issues may contribute to such outcomes. This problem needs to be studied further. Because of this, it is important to carefully create cost functions with optimization sub-objectives of all stakeholders, and, in particular, to use levelizedand not marginal-cost functions. This is critical for aligning technical and economic objectives, and for ultimately making TEM a long-term viable solution. We discuss and illustrate the interdependence of performance metrics used in this report, on one hand, and the sub-objectives of different entities participating in TEM, on the other. We explain how the TEM outcomes depend on whether relevant constraints are modeled as hard constraints, or they are included as part of objective functions. This choice has major impacts on the resulting customer comforts, revenues, 
social welfare, network characteristics, and voltage profile, in particular. Only in Case 4, when stakeholders are price makers proactively exchanging and responding to dynamic prices, does it becomes possible to arrive at a win-win solution in which sub-objectives of the stakeholders and the system align. Notably, we show that the grid owners and operators (utilities) must be treated as stakeholders with well-defined sub-objectives, and not as constraints in the TEM. We show how different performance metrics chosen by the grid could significantly affect the performance metrics of interest. These observations are illustrated using the IEEE 8500 bus distribution network with lots of solar PVs.

Major recommendation: Given the above technical and economic issues related to effective TEM implementation, we propose that the industry should reconsider using the peak-load pricing because of its basic role in reconciling losers/winners issues. Peak load pricing indirectly gives the right incentives to participate in dynamic pricing because this results in overall longer-term efficiency and lower cost. In particular we observe that utilities/grid operators have major roles to play in the changing industry and that their revenues can be increased by providing the right TEM incentives. The basic idea underlying peak load pricing (PLP) is that longer-term ex ante tariffs can be designed based on the expected load profile and the expected system-level value provided by each particular stakeholder [29]. This can be implemented as a multi-step TOU tariff known ahead of time by the grid users. The actual computation of this multi-step TOU tariff should be based on the best estimate and predictions of the likely load, LMPs and their cumulative effects on the need for new capital investments. However, in order to incentivize customers and other stakeholders to consume/produce/store at the best times for the expected system-level social welfare, the PLP should be designed so that it reflects the tradeoff between break-even cumulative marginal cost reduction and the incremental cost of investing in new hardware and smart controls.

In the context of the IEEE 8500 grid studied, it becomes possible to design PLP tariffs so that the expected value of solar PVs, storage, capacitor banks and utility voltage regulation becomes part of tariffs. The simplest way of thinking about PLP vs. LMP is to think of PLP as an ex ante tariff based on temporal prices, such as TOU or LMPs. Notably, this value can be extracted only if an optimization is used rather than power flow analysis. Fundamental to creating these PLPs in an ex ante manner is that they must be based on MPC decision making rather than static decision making. Forecasting grid voltages based on DER forecast response tied to forecast LMPs rests on multiple forecasts over time. Much benefit, measured in terms of expected social welfare and the amount of clean power used, can be obtained from such an approach. We point out that LMP-based pricing is generally insufficient for recovering the capital cost of investments into the hardware needed to implement TEM. This is particularly true of investments in controllable equipment and storage.

\section{Acknowledgments}

This MIT contribution to the NIST Phase II Transactive Energy Management Challenge is based on our past and on-going work. Most of the material directly draws on the EESG@MIT Working paper entitled "Toward Operationally Feasible and Efficient Integration of Distributed Energy Resources (DERs)" [5]. The authors acknowledge the collaborative funding provided by NIST to Carnegie Mellon University which led to the design of Smart Grid in a Room (SGRS) scheduler, publicly available as a BSD-3 software. Many discussions with NIST researchers are greatly appreciated. The authors also acknowledge the use of NETSS AC OPF provided for research purposes only. The help by several MIT Supercloud folks in setting up the MIT SEPSS is also greatly appreciated. Last, 
but not least, partial funding for this work was provided by NIST under an MIT research project and by ARPA-E under the NODES project to MIT Lincoln Laboratory. This project is supported by the Department of Energy under Air Force Contract No. FA8721-05-C-0002 and/ or FA8702-15-D0001. Any opinions, findings, conclusions or recommendations expressed in this material are those of the authors and do not necessarily reflect the views of the Department of Energy.

\section{8. $\quad$ References}

[1] Ilic, M.D., Jaddivada, R., \& Miao, X., Scalable Electric Power System Simulator, Innovative Smart Grid Technologies-Europe (IEEE ISGT Europe), 2018.

[2] Ilic et al, Collaborative NIST-CMU Project Progress report, 2015.

[3] Ilic et al, Collaborative NIST-CMU Project Progress report, 2016.

[4] MIT EESG white paper/ NIST Project funding.

[5] Ilic, M. D., \& Jaddivada, R. (2018). Multi-layered interactive energy space modeling for nearoptimal electrification of terrestrial, shipboard and aircraft systems. Annual Reviews in Control.

[6] Ilic, M.D. \& Jaddivada, R., "Toward Operationally-Feasible and Efficient Integration of

Distributed Energy Resources (DERs)”, EESG@MIT Working Paper No. R-WP-11-2018.

[7] Ilic et al, Collaborative NIST-CMU Project Progress report, 2017.

[8] Ilic et al, Collaborative NIST-MIT Project Progress report 1, 2017.

[9] Prout, A., Kepner, J., Michaleas, P., Arcand, W., Bestor, D., Bergeron, B., \& Mullen, J. (2015, September). Enabling on-demand database computing with MIT Supercloud database management system. In High Performance Extreme Computing Conference (HPEC), 2015 IEEE (pp. 1-6). IEEE. [10] Reuther, A., Kepner, J., Arcand, et.al. (2013). LLSupercloud: Sharing HPC Systems for Diverse Rapid Prototyping. In High Performance Extreme Computing Conference (HPEC), 2013 IEEE (pp. 1-6).

[11] Dahmann, Judith S., and Katherine L. Morse. "High level architecture for simulation: An update." In Distributed Interactive Simulation and Real-Time Applications, 1998. Proceedings. 2nd International Workshop on, pp. 32-40. IEEE, 1998.

[12] Burns, M., Song, E., Holmberg, D., The Transactive Energy Challenge Abstract Component Model, NIST Special Pub 1900-602, 2018.

[13] M.D. Ilic, R. Jaddivada, Transactive Energy Market with implementable bids, Innovative Smart Grid and Technologies, Washington, February 2018.

[14] Ilic, M. D. (2011). Dynamic monitoring and decision systems for enabling sustainable energy services. Proceedings of the IEEE, 99(1), 58-79.

[15] M. D. Ilic, R. Jaddivada, X. Miao, N. Popli, "Generalized Physics-Based Multi-Functional Droops of Controllable Generation and Demand", EESG@MIT Working Paper No. R-WP-3-2018. [16] Zhou, X., Dall'Anese, E., Chen, L., \& Simonetto, A. (2017). An incentive-based online optimization framework for distribution grids. IEEE transactions on Automatic Control.

[17] Standard, A. N. S. I. C84. 1-1982. American National Standard for Electric Power Systems and Equipment-Voltage Ratings $(60 \mathrm{~Hz})$.

[18] Paul Kleindorfer, Economic regulation under distributed ownership: The case of electric power transmission. 2014.

[19] Schneider, Kevin P., and Jason C. Fuller. "Voltage control devices on the IEEE 8500 node test feeder." In Transmission and Distribution Conference and Exposition, 2010 IEEE PES, pp. 1-6. IEEE, 2010. 
[20] M. D. Ilic, R. Jaddivada, N. Popli, “Transactive Energy Market Architectures for Providing Load- Following Service,” EESG Working Paper No. R-WP-2-2018.

[21] Barrager, Stephen M., and Edward G. Cazalet. Transactive energy. Reston: Public Utilities Reports, Inc., 2016.

[22] Ilic, M., Jaddivada, R., Miao, X., Popli, N., "Toward multi-layered MPC for complex electric energy systems", Chapter in "Handbook of MPC", Birkhauser, 2018.

[23] Jaddivada, R., \& Ilic, M.D., "A Distribution Management System for Implementing Synthetic Regulation Reserve”, IEEE North American Power Symposium (NAPS), September 2017, Morgantown, USA.

[24] J. Y. Joo and M. D. Ilić, "Adaptive load management (ALM) in electric power systems," 2010 International Conference on Networking, Sensing and Control (ICNSC), Chicago, IL, 2010, pp. 637-642, doi: 10.1109/ICNSC.2010.5461584.

[25] Ilic, M., Cvijic, S., Lang, J. H., Tong, J., \& Obadina, D. (2015). Operating beyond today's PV curves: Challenges and potential benefits. In Power \& Energy Society General Meeting, 2015 IEEE (pp. 1-5).

[26] Holmberg, D., Burns, M., Bushby, S., Gopstein, A., "Transactive Energy Challenge Phase II Scenario," NIST Tech. Note 2021, 2018.

[27] CAISO real-time market prices for July 6, 2017, Tucson LMP five-minute clearing price data (2017) "SG_LNODE13A.player" [Available Online: https://github.com/pnnl/tesp/tree/master/examples/players]. [28] M.D. Ili'c, R. Jaddivada, "Toward Operationally-Feasible and Efficient Integration of Distributed Energy Resources (DERs)”, EESG Working Paper No. R-WP-11-2018, April 2018. [29] Joskow, P. L., \& Wolfram, C. D. (2012). Dynamic pricing of electricity. American Economic Review, 102(3), 381-85. 


\title{
Section 6 Vanderbilt Team Report: \\ Cyber-Physical Systems Wind Tunnel for Transactive Energy (CPSWT-TE) Design Studio
}

\author{
Himanshu Neema \\ Vanderbilt University, Nashville, TN
}

\begin{abstract}
In the last decade, we have witnessed a rapid evolution of technologies in energy generation as well as distribution. In addition, distributed energy resources (DER) have increased in use causing the utilities to upgrade their business models. Transactive Energy (TE) approaches aim to increase the flexibility of grid operations under novel DERs (e.g. solar panels and household large-scale batteries), as well as to better manage the grid under varying demand and supply conditions. To enable TE, many stakeholders, such as utilities, prosumers, markets, distributors, and regulators need to work together in a collaborative manner, despite their different requirements and priorities. There are no co-simulation platforms that enable such collaborative experimentation. Enabling this kind of collaborative TE co-simulation requires not only a highly programmable and customizable co-simulation platform that supports modeling and simulation with a number of special-purpose simulators in the same integrated experiments, but also a rich collaboration platform where stakeholders can share and use models and experiments. In addition, tools and techniques are needed for proper versioning and storage of models and experiments, and for traceability of experiments and experiment results.

In this research we developed a cloud-deployed, web-based, model-based co-simulation framework for transactive energy and integrated it with the CPS virtual organization collaboration platform. This enables users to create models and experiments, share them collaboratively in a version-controlled system, and run experiments of transactive energy approaches all using a web-browser. A unique feature of this platform is that its implementation architecture enables users of the platform to extend it themselves for features that are relevant to them. Another capability of the platform enables utilities to experiment in a collaborative manner even when the models they are experimenting with are proprietary. Access rights enable sharing of proprietary models, which might be in a binary format, only with those who are part of the collaboration for the experiments being conducted.
\end{abstract}




\section{Table of Contents}

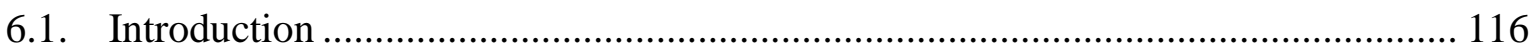

6.2. CPSWT-TE Design Studio Research Approach ............................................ 118

6.3. CPSWT-TE Design Studio Core Architectural Components............................... 120

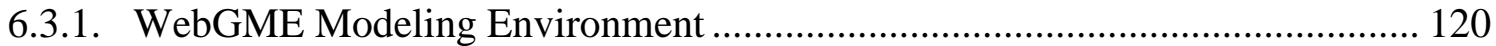

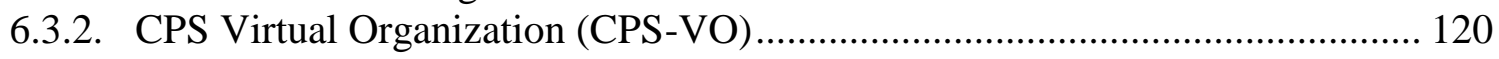

6.3.3. Experimental Runtime Cloud ..................................................................... 121

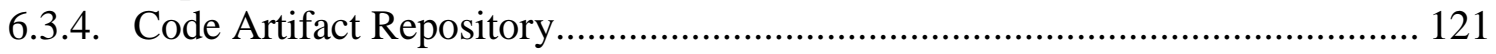

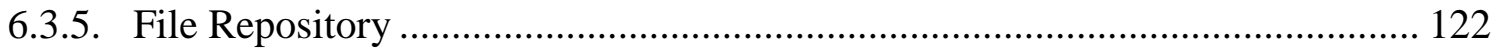

6.4. CPSWT-TE Design Studio Experiment Development Workflow ......................... 123

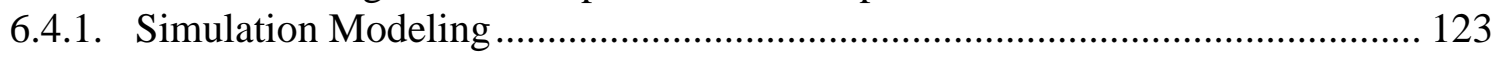

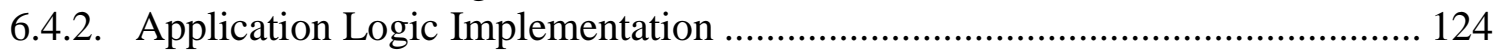

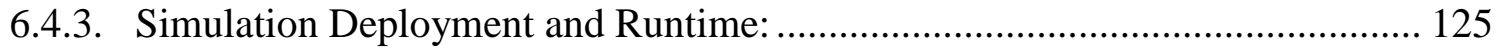

6.5. GridLAB-D Modeling and Simulation Design Studio ....................................... 126

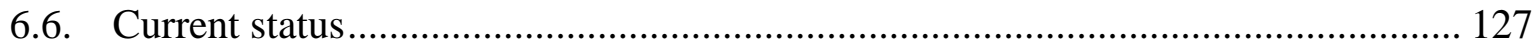

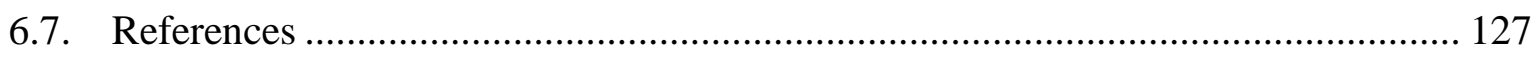




\subsection{Introduction}

Realistic TE simulation results can only be achieved by simultaneous consideration of many different TE concerns that arise from utilities, energy distributors, power markets, regulators, and consumers, as well as dynamical aspects that arise due to tight interaction among physical domains such as electrical, mechanical, thermal, structural, and cyber. These concerns are shown in Figure Vanderbilt-1.

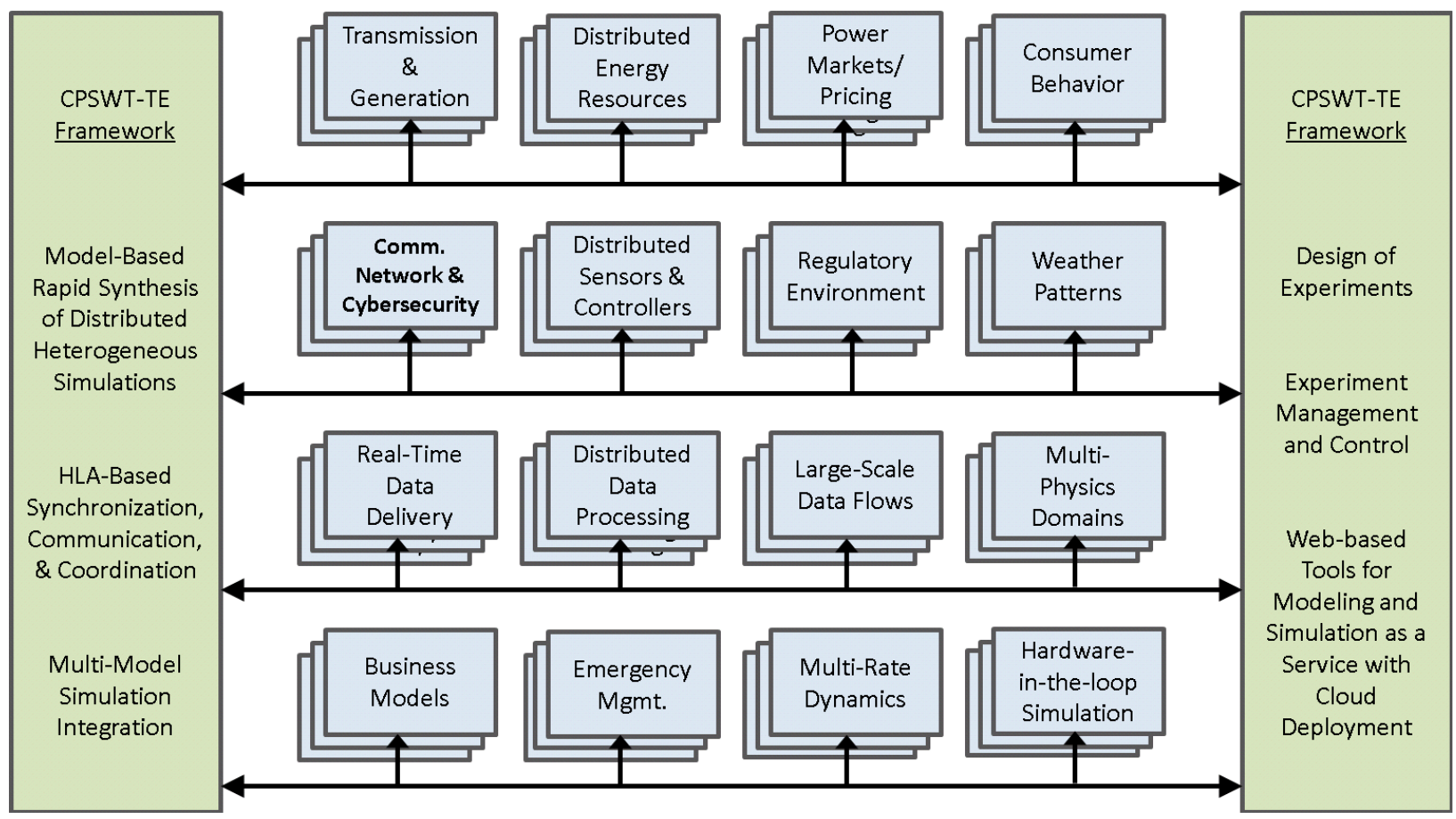

Figure Vanderbilt-1 Transactive Energy Concerns Targeted in CPSWT-TE

Evaluating realistic TE scenarios with simultaneous consideration of these concerns is a highly complex and computationally expensive challenge. Vanderbilt University is developing a web-based modeling \& co-simulation environment [1] that relies on the IEEE High-Level Architecture (HLA) standard [2] and provides cloud-backed experimentation and analysis services. Using this platform, called the Cyber-Physical Systems Wind Tunnel for Transactive Energy (CPSWT-TE) Design Studio [3], TE evaluators will be able to perform most of their modeling and experimentation tasks using only a web-browser.

In this research, we describe the novel CPSWT-TE platform that is available online for anyone to use. The CPSWT-TE platform was developed using a general-purpose heterogeneous simulation integration framework, developed at our institute, that has been used by a variety of government agencies such as AFOSR, AFRL, DoD, NIST, and companies like BAE systems, Alion Science, etc. The CPSWT-TE platform uses this general-purpose integration framework as the basic building block for supporting TE specific co-simulations. 
The key aspect of this platform is that it is web-based with a cloud backend that enables users to experiment with it simply using a web-browser. In addition, the platform provides a model-based simulation integration framework that enables users to create co-simulation models for transactive energy experiments using the web-browser and conduct experiments with it in the cloud. The platform has been integrated with the CPS virtual organization (CPS-VO) collaboration platform, which enables users to create models and experiments in a collaborative environment and share them in a version controlled and authenticated manner. Another key aspect of the platform is that it has been implemented with an architecture that enables users to extend its functionality themselves for features that are relevant to them. In addition, users can experiment with models that might be proprietary. The access control mechanisms enable sharing of models only for those who are part of the collaboration for the experiments being conducted. At the same time, the proprietary models could be added to the platform in binary format for additional privacy. For example, these features would enable utilities to experiment with their proprietary business models with different grid models and stakeholders in a collaborative manner, while still keeping their proprietary models private.

The primary goals of this research are as follows:

1. Create a baseline web-based co-simulation platform that enables teams in the TE Challenge to experiment with simple transactive energy co-simulations.

2. Generically support simulation components written in $\mathrm{C}++$ or Java.

3. Support GridLAB-D simulation components for power-grid simulation.

4. Create an online environment where users can be registered, authenticated, and authorized for specific platform features such as modeling and experimentation.

5. Ensure users can upload their GridLAB-D models and other software code for simulations written in $\mathrm{C}++$ and Java.

6. Allow many experiments to be executed in parallel in the backend cloud environment.

7. Enable monitoring of experiments that are currently running.

8. Provision cloud computation resources to users in a first-in, first-out order.

9. Provide results of the experiments conducted to be downloaded from the platform.

10. Provide authenticated and partitioned persistence services for users to store on the online platform (i) data files such as models, results, and (ii) software code for their simulation components (released and versioned).

11. Extend platform for new functionality and simulation types. 


\subsection{CPSWT-TE Design Studio Research Approach}

Simulations provide a great tool for analyzing TE concerns for a variety of stakeholders in this domain such as utilities, energy distributors, researchers, power markets, regulators, and increasingly even consumers. However, the simulations of these systems are highly dynamic in nature and require tight integration of many interacting physical domains (e.g. electrical, mechanical, thermal, structural, and cyber) as well as power pricing, transactions, regulations, and behavior of consumers. Realistic results with simulations at the higher-level can be achieved only when these aspects are considered together. This presents unique problems for individual stakeholders who may not have the tools and expertise in various domains, or lack the necessary software and hardware infrastructure to carry out the simulations in a reasonable amount of time. Thus, in many situations, simulations are partitioned to evaluate only some of the aspects or ignore some aspects altogether. Cloudbased simulations can provide a solution to this problem, but only if they include finegrained and extensible modeling. Previously, we described an approach to support modeland cloud-based design and experimentation with an environment called CPSWT-TE [4] [1]. Here, we present more details of the architecture.

Many concerns of transactive energy, such as grid simulation, transmission, distribution, markets, regulatory environment, weather, cyber communication and control, and cyber security are evaluated using different special-purpose simulation tools. However, for integrated simultaneous evaluations, these special-purpose simulation tools need to be tightly integrated. This is a rather difficult task. Different tools not only differ in language for modeling and implementation, but also heavily in the semantics of the models used and their assumed models of computation [5]. We are developing the web-based CPSWT-TE environment [3] to address this problem.

Figure Vanderbilt-2 shows the overall architecture of the CPSWT-TE platform. The platform uses simulation Runtime that is based on the IEEE HLA standard [2]. The different simulation tools are made HLA-compliant and talk to each other and synchronize using HLA Run-Time Infrastructure (RTI) services. We have developed custom adapters for a number of simulation tools such as GridLAB-D [4], OMNeT++ [6], MATLAB/Simulink [7], Colored Petri-Nets [8], SUMO [9], Functional Mockup Units based on FMI-standard FMU-CS [10], Train Director [11], etc. Also, any simulation tool that has a Java or C++ API for controlling its logical time and interfacing for inputs and outputs can be integrated in the platform. 


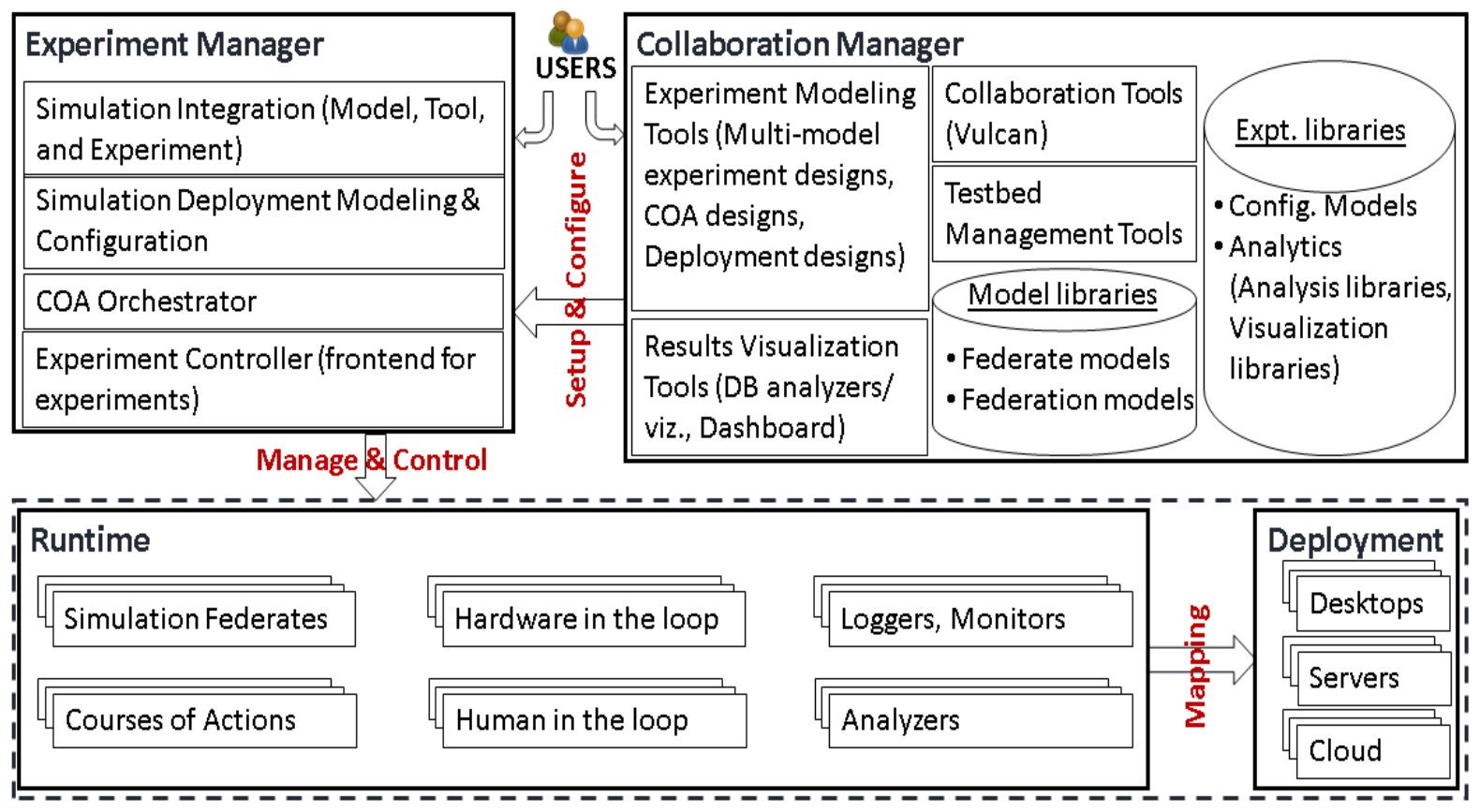

Figure Vanderbilt-2 CPSWT-TE Platform Architecture

In Figure Vanderbilt-2, simulation experiments can be executed on different Deployments. The facilities needed for creating integration models and configuring the experiments are provided by the Experiment Manager. It is a novel capability of the platform to support many alternative Courses of Action (COAs), i.e. paths of scenario execution. Many experiments use COAs to design What-if scenarios to capture workflows that are dependent on the actual ongoing simulation outputs and can modify the ongoing simulations to direct them in an alternative branch of execution. The Collaboration Manager uses an open-source Vulcan Framework [12] that provides a number of collaboration tools and services using distributed micro-services architecture and cloud deployments. 


\subsection{CPSWT-TE Design Studio Core Architectural Components}

In this section we present the architectural components of the CPSWT-TE Design Studio [3]. Figure Vanderbilt-3 illustrates the different architectural components of the Design Studio. These components are further described in the sections below.

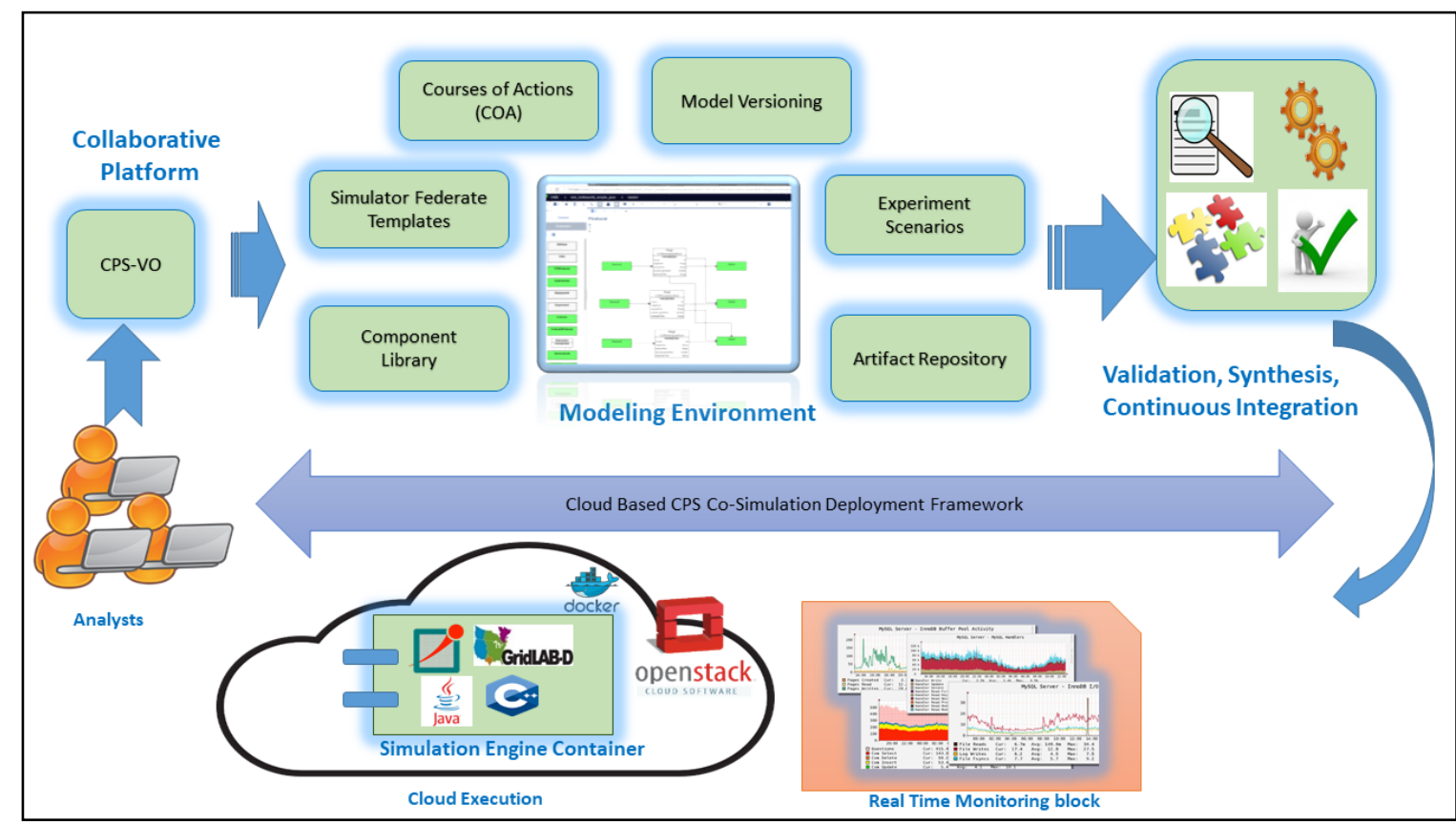

Figure Vanderbilt-3 CPSWT-TE Design Studio Architectural Components (Source: [14])

\subsubsection{WebGME Modeling Environment}

WebGME is a web-based modeling tool that provides a design environment to design HLA based distributed simulation models. WebGME is a generic modeling environment to build and develop meta-models and models, model interpreters and visualization [14].

In our CPSWT-TE Design Studio, a domain expert can extend existing meta-models for a collection of HLA federates by providing a meta-model for a new HLA federate. The HLA user uses the WebGME framework to develop model instances and configure them for experimental scenarios. After an HLA based distributed simulation experiment is constructed, code generator plugins are invoked to generate boilerplate code templates and simulation configuration artifacts. The code artifacts can be extended to bring in user specific application logic and algorithms.

\subsubsection{CPS Virtual Organization (CPS-VO)}

Cyber-Physical Systems - Virtual Organization [15] provides a web-based environment for CPS communities to collaborate, and share ideas on CPS designs and activities, as well as a sophisticated cloud environment for experiments using a set of shared "active resources", including user authentication and management functions and experiment configuration, control, and execution facilities to deploy cloud-based integrated simulations. Thus, the CPS- 
VO provides a web-based community platform for researchers and users to manage and deploy distributed simulation experiments on the experimental runtime cloud. Key features of the CPS-VO used in this design studio include:

- Resource scheduling for experiment deployment

- User authentication and management

- Experiment input and result collection

- Experiment control panel

\subsubsection{Experimental Runtime Cloud}

To support large scale deployment of HLA distributed simulation in a virtual environment, cloud computing technology offers tremendous benefits such as on-demand availability of large-scale resources to run long running and resource intensive simulations. Openstack is an open-source cloud computing framework that enables different users to utilize cloud computing resources. Our Design studio utilizes Vanderbilt University's Openstack-based cloud for running experiments. A pool of virtual machines is created to support user experimentation. These virtual machines are running Ubuntu-based 64-bit Linux operating systems. In the CPSWT framework, we are running the HLA federates encapsulated inside a Docker container [16], which utilizes Linux Container technology [17]. Our virtual machines are preconfigured to support the Docker runtime engine.

HLA federates are deployed as a Docker container, and the specification of the federate is defined in a federate descriptor configuration file. The configuration file is defined in a YAML format and includes specifics such as:

- The base Docker image to be utilized for the federate depending on the type of federate (e.g., C++, JAVA, OMNET++),

- File repository URLs for access to the simulation specific files,

- Storage of the output logs, and

- Simulation-specific environment variables.

\subsubsection{Code Artifact Repository}

A number of code artifacts are generated by the WebGME framework for the desired distributed simulation model. Using these code artifacts, the users can design their application logic for the HLA federates. These code artifacts are then used during the experimental run of the HLA simulations. A central storage location is thus required for managing and providing storage for these code artifacts. A code artifact repository that we have selected in our design is the Apache Archiva [18] code repository. Archiva supports Maven [19] build system binary artifacts. The Maven build system compiles and packages code artifacts that are ready for distribution. Each of these artifacts has a unique name, version number, and group or organization name. Maven artifacts also contain information about the various dependencies that may be needed for execution purposes. All the code and build artifacts are hosted in the centralized Archive repository, which is accessible to both the system developers and the execution platform that is responsible for running the distributed simulation experiment. Archiva supports the creation of users using RESTful protocol which is utilized to provide user access management. 


\subsubsection{File Repository}

A file repository, unlike the code artifact repository, is responsible for managing additional artifacts required for the execution of the distributed simulation experiment. These include simulation runtime model files specific to a given simulation environment. For example, the GridLAB-D simulation environment uses simulation models that are expressed in a format that is packaged as a GridLAB-D model file (.glm). The file repository also hosts the experiment specification as declared in the experiment form sheet of the WebGME distributed simulation editor. The experiment specification is shown in Figure Vanderbilt-4.

The experiment specification includes the specification of a set of federates that comprise a federation. It also specifies the network configuration of the individual federates comprising the HLA distributed simulation. Further, it specifies the Courses-of-Action to include in the experiments. The file repository also hosts output files generated from an experiment. The output files consist of experiment-generated $\log$ files, analysis graphs and plots, and reports as to whether an experiment completed successfully or failed with error logs. The file-hosting server supports HTTP file service access to upload, download, modify and delete operations on the files in the repository.

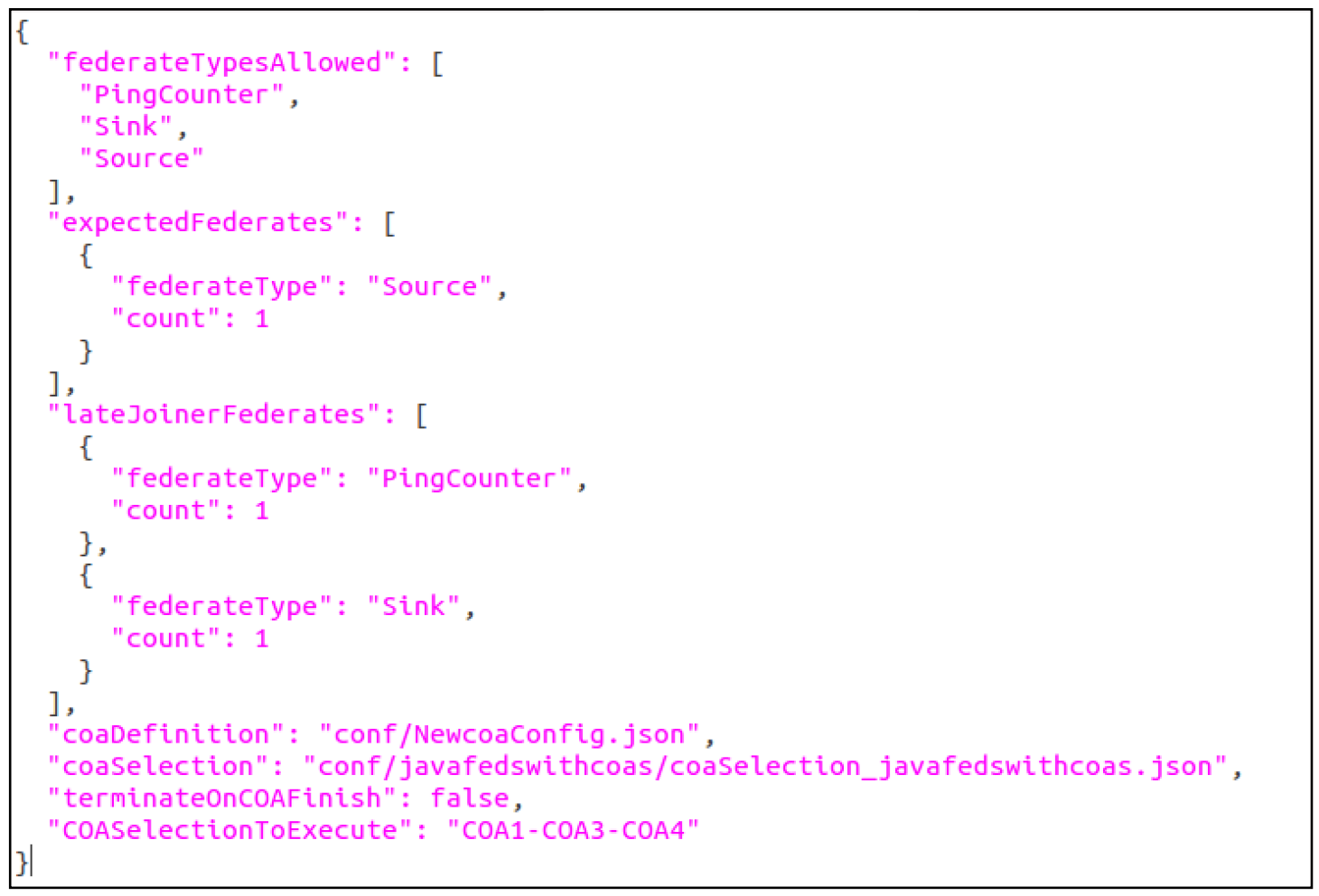

Figure Vanderbilt-4 Experiment Specification Example 


\subsection{CPSWT-TE Design Studio Experiment Development Workflow}

In this section we will discuss the primary workflow in the design development and experimental execution of distributed simulations in the CPSWT-TE Design Studio. Figure Vanderbilt-5 illustrates three main phases of the design studio workflow, (1) Simulation Modeling, (2)Application Logic Implementation, and (3) Simulation Deployment and Runtime. In the sections below, we will cover details about each of these workflow phases.

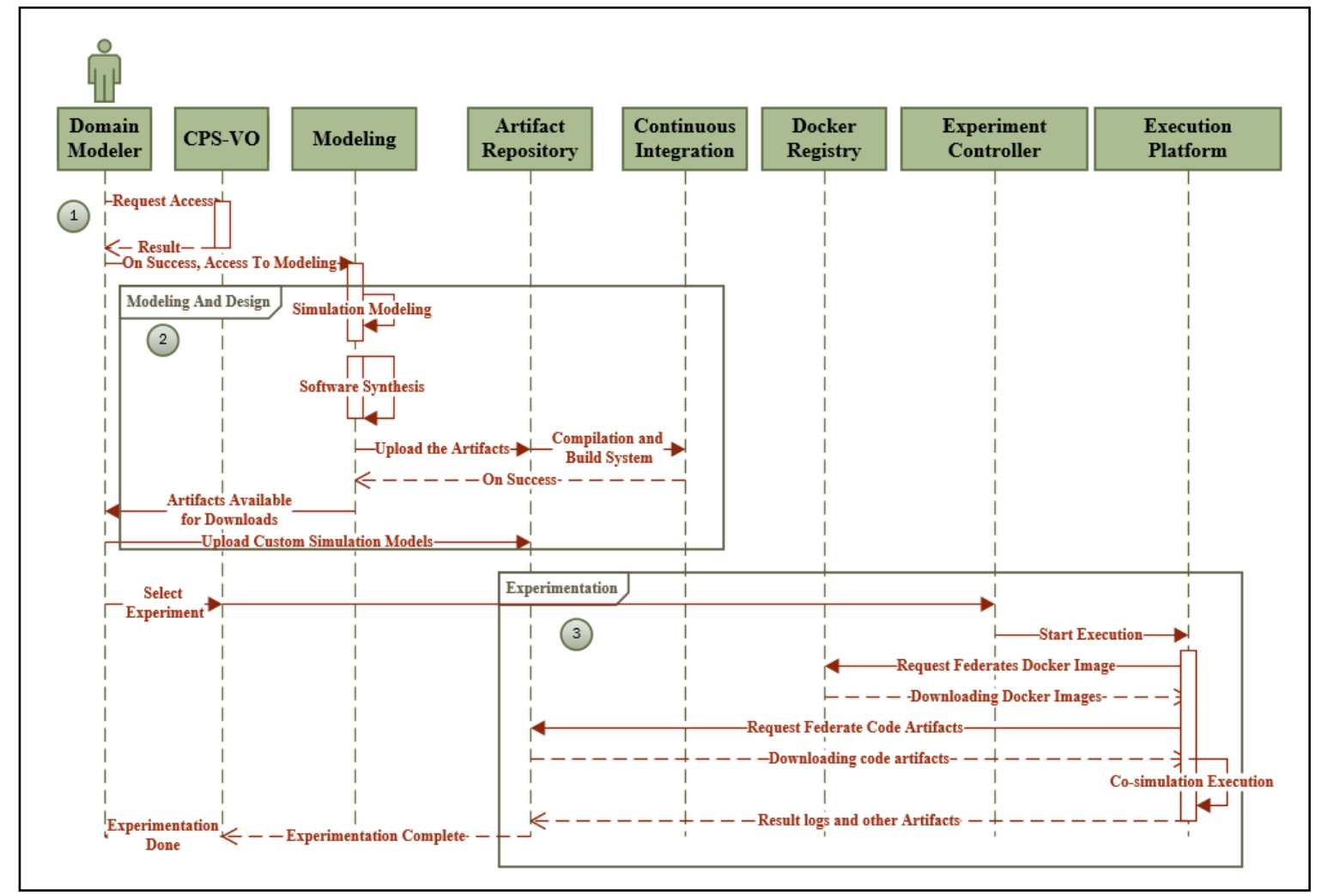

Figure Vanderbilt-5 CPSWT-TE Design Studio Experiment Development Workflow (Source [13])

\subsubsection{Simulation Modeling}

Experimenters/analysts describe their simulation models in the WebGME modeling environment utilizing domain specific models of different HLA federates. These models consist of C++, Java, and GridLAB-D specific federate implementations. The analyst selects the suitable language bounded federate model based on their execution and language platform requirement. The analyst also specifies interactions that are supported by different federates in the HLA federation. Interactions specify the types of messages that federates subscribe to or publish in an HLA federation.

The analyst also specifies the experiment for a given simulation run. The specification may include the step size the simulation needs to proceed at, total simulation runtime, name of the HLA federation, etc. 


\subsubsection{Application Logic Implementation}

Once the simulation modeling is complete the analyst can then run special executors. In WebGME nomenclature, executors are called plugins. Plugins are responsible for performing special functions based on the modeling execution needs. The main functionalities performed by the plugins are presented here.

\section{Code Generators}

The code generator plugin is responsible for generating the necessary base code artifacts for the HLA simulation. The base code artifacts primarily consist of two main modules:

- Federate Base classes: These define the language-specific base code implementation of a given federate.

- Federate RTI classes: These specify the interaction types for the federation.

\section{Experiment Configuration}

Plugins also generate experiment-specific deployment files, which contain information about the different federates comprising the federation, and the type of federates such as $\mathrm{C}++$, Java, GridLAB-D. These deployment files are uploaded to the file repository as discussed earlier.

\section{Application Programming Templates}

Plugins also generate the application programming template, which the analyst can use to start developing application logic. The template contains all the programming glue code that is required to have an executable distributed simulation. This allows the analyst to only be concerned about building the application logic for the HLA federate, without being concerned about setting up the various toolchain dependencies and build script.

\section{Packaging and Deployment}

Once the code artifacts are generated, they are passed to a build system software block. The build system handles the following:

- Compiles and builds the code artifacts.

- Stores the code artifacts to the Archiva code repository.

\section{Compilation and Testing Scripts}

Compilation and testing of the HLA-based federation is a crucial step in the execution of the distributed simulation. To ease this development activity, the plugin also generates a set of compile, build and execution scripts. As in the case of the CPSWT-TE runtime environment, we run distributed simulation federates inside a Docker container. A preconfigured docker image is made available to the analyst, by which one can create a running docker container to test the runnable federate and check simple functionality such as whether the federate can join and exit a federation successfully.

The plugin thus creates the following files to ease this phase of development:

1. compile.sh: script to compile the federate codes.

2. run.sh: script to run the federate inside a test docker container.

3. deploy.sh: script to deploy the code artifacts to the central Archiva repository. 
4. docker-compose.yml: script to configure and instantiate the test docker container with the input and output folders with the necessary federate code that needs to be tested.

\subsubsection{Simulation Deployment and Runtime:}

After the analyst has created the experiment specification and is ready to run the simulation experiment, the following experimentation procedure is followed. The user logs into the CPS-VO experimentation portal as shown in Figure Vanderbilt-6.

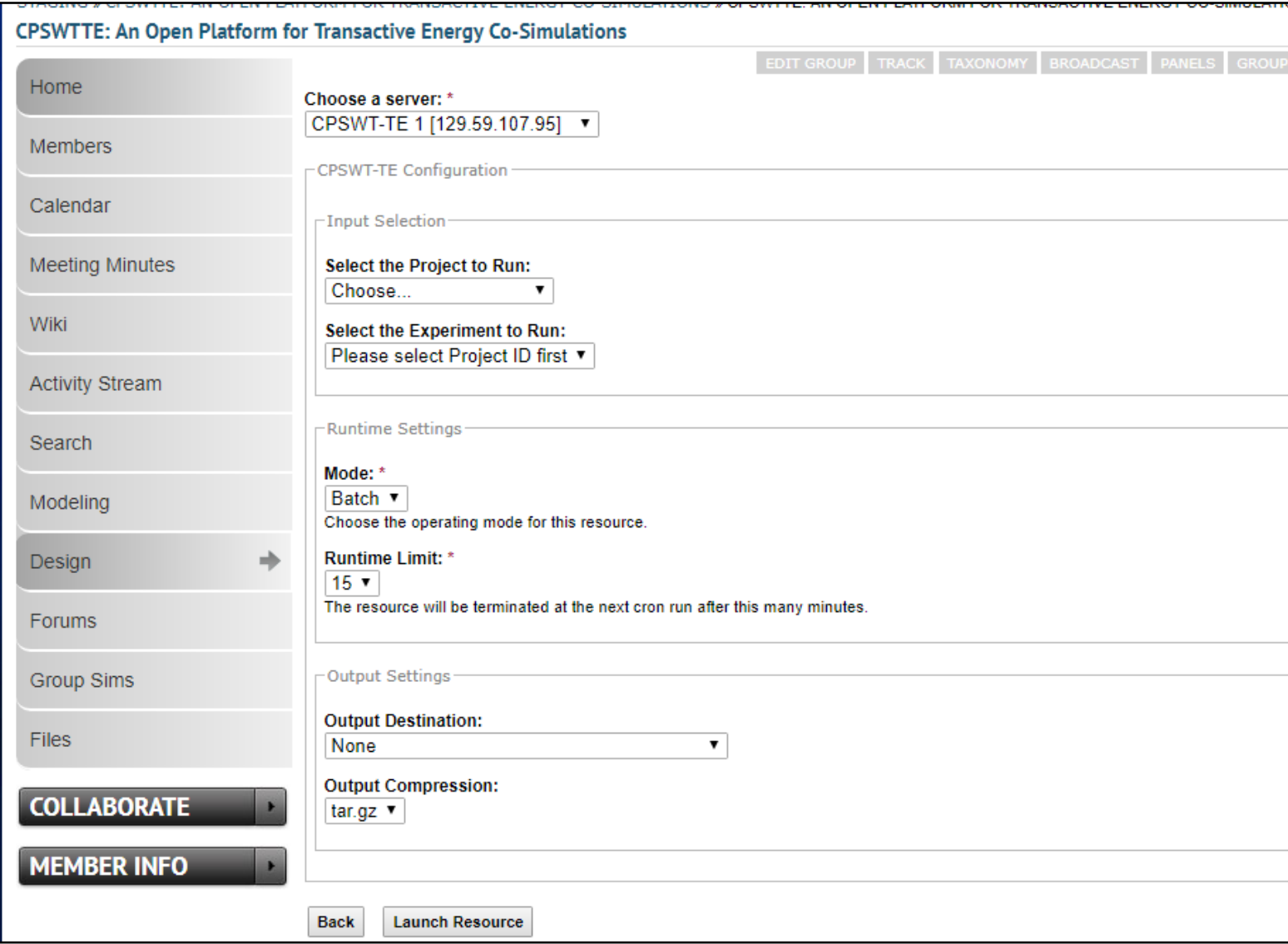

Figure Vanderbilt-6 CPS-VO Experimentation Interface for the CPSWT-TE Design Studio

After logging in, the user selects a target server on which to deploy the experiment from the pool of experimentation servers. From the input selection drop down option, the simulation project is selected. A user selects a simulation project from among the simulation projects currently shared in the WebGME portal. Each project can have a set of experimentation scenarios. The user can select the type of experiment that needs to be run on the server. Once both the project and the experiment have been selected, the user can specify a maximum runtime limit, which is the duration beyond which the experiment will be terminated. The experiment specific results are gathered and stored on the CPS-VO. 


\subsection{GridLAB-D Modeling and Simulation Design Studio}

The GridLAB-D design studio [20] allows a user to work with and simulate GridLAB-D model files. GridLAB-D is a new power distribution system simulation and analysis tool that provides valuable information to users who design and operate distribution systems, and to utilities that wish to take advantage of the latest energy technologies. It incorporates the most advanced modeling techniques with high-performance algorithms to deliver the best in enduse modeling. GridLAB-D is coupled with distribution automation models and software integration tools for users of many power system analysis tools. Below are the important points about this design studio:

1. The design studio works with GridLAB-D model files (with .glm extension).

2. The installed version of GridLAB-D is 4.0.0 and it runs in a 64-bit Ubuntu Linux Virtual Machine on the server.

3. A familiarity with GridLAB-D is expected for users to use this design studio.

4. The design studio's front-end is based on WebGME modeling environment.

5. It uses WebGME version 2.24.0.

6. Some familiarity with WebGME is useful.

7. References for GridLAB-D simulator, WebGME modeling environment, and the access page for the design studio are provided at the design studio main page.

This design studio allows working with GridLAB-D models in various ways and also executing them on the server. Before experimenting with the design studio, we recommend reading further details and various use-cases about it, which are described next. There are three main use-cases in which this design studio can be used:

1. Import a GridLAB-D model file (.glm) into the design studio to graphically visualize the Grid model.

2. $\quad$ Edit the current GridLAB-D model file and export as .glm files.

3. Execute a GridLAB-D model file on the server using the GridLAB-D simulator.

For more details about this design studio, please refer to its homepage [20]. 


\subsection{Current status}

Considerable progress has been made in the past few months in creating the baseline webbased co-simulation platform. The $\mathrm{C}++$, Java, and GridLAB-D components are already integrated in the backend platform. Web-based modeling technology and the necessary modeling language have been developed along with the tools needed to exercise the experiment models as runnable experiments in the backend. We have also created tools needed by users to exercise the platform. The user can customize simulation code and upload the binary artifacts into the cloud backend. In order to separate the code artifacts in userspecific storage areas some more work is needed. We have added five dedicated servers on the cloud environment for users to perform experiments. When new experiments are launched, the appropriate available server can be selected and used for up to 30 minutes per experiment. If none of the servers are currently available due to ongoing experiments, then the user waits until one becomes available.

In the future, we plan on implementing an automatic queuing mechanism, where the user can simply launch their experiments and they will be automatically queued and executed in the first-in-first-out order as soon as a server becomes available. Both of the design studios described in the report are available at the references below:

1. Cyber-Physical Systems Wind Tunnel for Transactive Energy (CPSWT-TE Design Studio) [3]

2. GridLAB-D Modeling and Simulation Design Studio [20]

\subsection{References}

1. Neema, H., J. Sztipanovits, M. Burns, and E. Griffor, "C2WT-TE: A Model-Based Open Platform for Integrated Simulations of Transactive Smart Grids", 2016 Workshop on Modeling and Simulation of Cyber-Physical Energy Systems, Vienna, Austria, 04/2016. 2. IEEE, "IEEE standard for modeling and simulation (m\&s) high level architecture (hla)--framework and rules," IEEE Std 1516-2010 (Revision of IEEE Std 1516-2000), pp. 1-38, 2010.

3. Cyber-Physical Systems Wind Tunnel for Transactive Energy (CPSWT-TE Design Studio): https://cps-vo.org/group/cpswtte

4. Neema. H., "Large-Scale Integration of Heterogeneous Simulations", Ph.D. Dissertation, Jan. 2018.

5. E. A. Lee and A. Sangiovanni-Vincentelli, "A framework for comparing models of computation," IEEE Transactions on Computer-Aided Design, vol. 17, 1998.

6. A. Varga, "The omnet++ discrete event simulation system," in Proceedings of the European simulation multiconference (ESM 2001), 2001, vol. 9.

7. Mathworks. (August 30, 2016). Matlab. Available: http://www.mathworks.com/products/matlab/

8. K. Jensen, L. M. Kristensen, and L. Wells, "Coloured petri nets and cpn tools for modeling and validation of concurrent systems," International Journal on Software Tools for Technology Transfer, vol. 9.3-4, pp. 213-254, 2007. 
9. D. Krajzewicz, J. Erdmann, M. Behrisch, and L. Bicker, "Recent development and applications of sumo-simulation of urban mobility," International Journal of Advanced System Measurement, vol. 5, no. 3-4, pp. 128-138, 2012.

10. H. Neema et al., "Model-based integration platform for fmi co-simulation and heterogeneous simulations of cyber-physical systems," in Proceedings of the 10th International Modelica Conference, Lund, Sweeden, 2014, pp. 232-245.

11. Train director. (2017). Available: www.backerstreet.com/traindir

12. Vulcan. (2017). Available: https://vulcan.isis.vanderbilt.edu/

13. Yogesh Barve, Himanshu Neema, Stephen Rees, and Janos Sztipanovits, "Towards a Design Studio for Collaborative Modeling and Co-Simulations of Mixed Electrical Energy Systems." Proc. of the 3nd International Workshop on Science of Smart City Operations and Platforms Engineering, (April 2018).

14. Maroti, M., R. Kereskenyi, T. Kecskes, P. Volgyesi, and A. Ledeczi, "Online Collaborative Environment for Designing Complex Computational Systems," The International Conference on Computational Science (ICCS 2014), Cairns, Australia, Elsevier Procedia, 06/2014.

15. Cyber-physical systems virtual organization. Available: https://cps-vo.org.

16. Docker - http://www.docker.comhttp://www.docker.com/

17. LXC - https://linuxcontainers.org/https://linuxcontainers.org/

18. Apache Archiva: https://archiva.apache.org/index.cgi.

19. Apache Maven Project: https://maven.apache.org/.

20. GridLAB-D Modeling and Simulation Design Studio: https://cpsvo.org/group/gridlabd 


\section{Appendix A TE Demonstration Projects in North America}

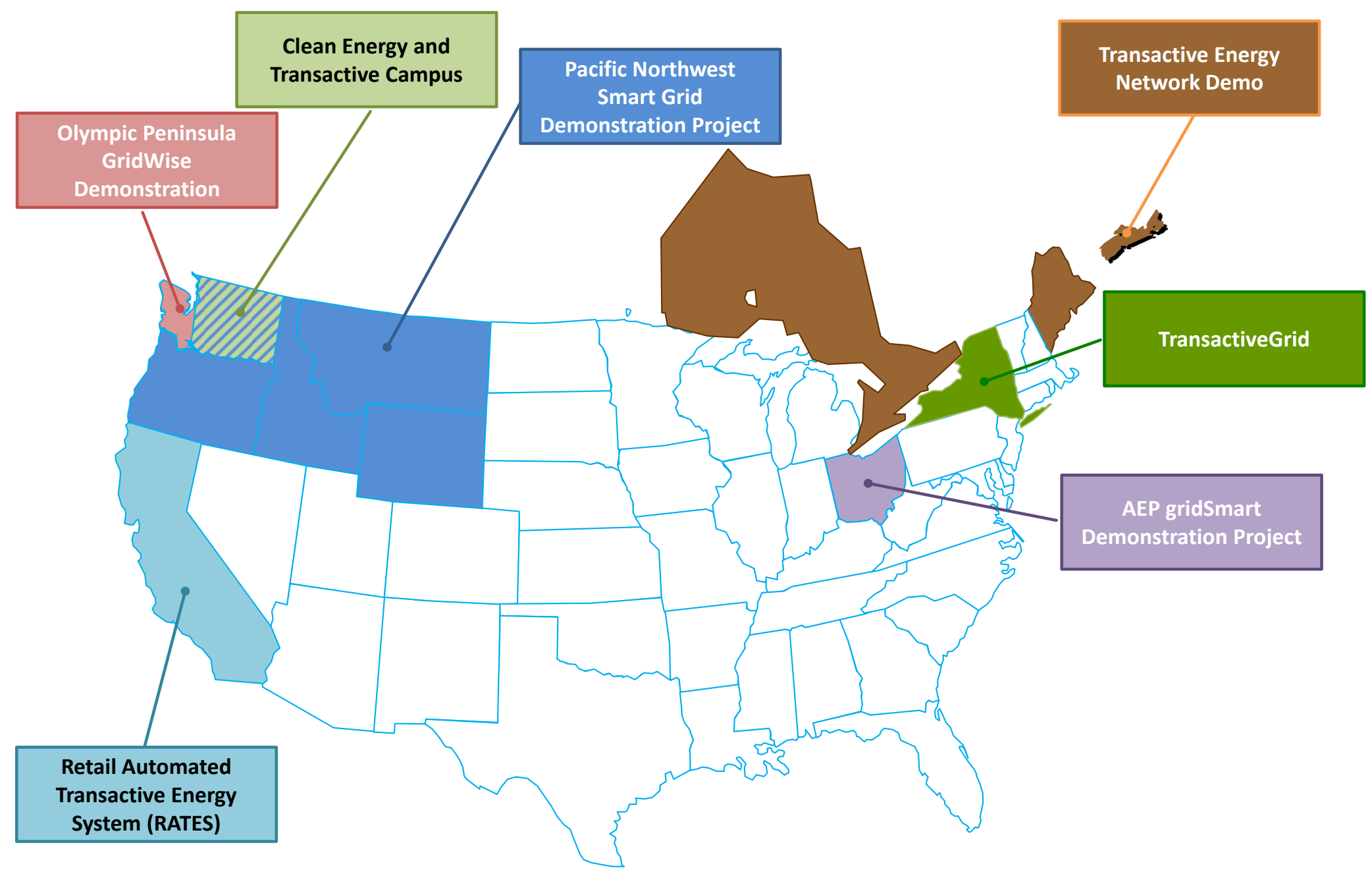




\begin{tabular}{|c|c|c|c|c|}
\hline Project & Description & Goal/Scope & Key Participants & Timing \\
\hline $\begin{array}{l}\text { Olympic } \\
\text { Peninsula } \\
\text { GridWise } \\
\text { Demonstration }\end{array}$ & $\begin{array}{l}\text { DOE-funded demonstration } \\
\text { project managed by PNNL. } \\
\text { Focused on testing of } \\
\text { automated two-way internet- } \\
\text { based communications } \\
\text { between the grid and } \\
\text { distributed resources, with } \\
\text { resources being dispatched } \\
\text { based on economic price } \\
\text { signals. }\end{array}$ & $\begin{array}{l}\text { - "Show that a common communications framework } \\
\text { can enable the economic dispatch of dispersed } \\
\text { resources and integrate them to provide multiple } \\
\text { benefits } \\
\text { - Gain an understanding of how these resources } \\
\text { perform individually and when interacting in near } \\
\text { real time to meet common grid-management } \\
\text { objectives } \\
\text { - Evaluate economic rate and incentive structures that } \\
\text { influence customer participation and the distributed } \\
\text { resources they offer"4 }\end{array}$ & $\begin{array}{l}\text { - PNNL } \\
\text { - Bonneville Power } \\
\text { Administration } \\
\text { - Public Utility } \\
\text { District \#1 of } \\
\text { Clallam County } \\
\text { - City of Port } \\
\text { Angeles } \\
\text { - Portland General } \\
\text { Electric }\end{array}$ & $\begin{array}{l}2004- \\
2007\end{array}$ \\
\hline $\begin{array}{l}\text { Pacific } \\
\text { Northwest } \\
\text { Smart Grid } \\
\text { Demonstration } \\
\text { Project }\end{array}$ & $\begin{array}{l}\text { DOE-funded demonstration } \\
\text { project managed by PNNL. } \\
\text { Implemented one of the } \\
\text { world's first transactive } \\
\text { coordination systems. } 25 \\
\text { separate asset systems were } \\
\text { incorporated into the } \\
\text { transactive network, } \\
\text { receiving supply, demand } \\
\text { and cost signals. }\end{array}$ & $\begin{array}{l}\text { - "Create the foundation for a sustainable regional } \\
\text { smart grid that continues to grow after the } \\
\text { completion of this demonstration project. } \\
\text { - Develop and validate an interoperable } \\
\text { communication and control infrastructure using } \\
\text { incentive signals to coordinate a broad range of } \\
\text { customer and utility assets, including demand } \\
\text { response, distributed generation and storage, and } \\
\text { distribution automation; engage multiple types of } \\
\text { assets across a broad, five-state region; and extend } \\
\text { from generation through customer delivery. } \\
\text { - Measure and validate smart grid costs and benefits } \\
\text { for customers, utilities, regulators, and the nation, } \\
\text { thereby laying the foundation of business cases for } \\
\text { future smart grid investments. } \\
\text { - Contribute to the development of standards and } \\
\text { transactive control methodologies for a secure, } \\
\text { scalable, interoperable smart grid for regulated and } \\
\text { non-regulated utility environments across the nation. }\end{array}$ & $\begin{array}{l}\text { - PNNL } \\
\text { - IBM } \\
\text { - QualityLogic } \\
\text { - } \text { 3TIER (now } \\
\text { Vaisala) } \\
\text { - Alstom Grid } \\
\text { - Bonneville Power } \\
\text { Administration } \\
\text { (BPA) }\end{array}$ & $\begin{array}{l}\text { December } \\
2009- \\
\text { June } 2015\end{array}$ \\
\hline
\end{tabular}

${ }^{4}$ Pacific Northwest GridWise ${ }^{\mathrm{TM}}$ Testbed Demonstration Projects. Part I. Olympic Peninsula Project. Pacific Northwest National Laboratory. October 2007. http://www.pnl.gov/main/publications/external/technical_reports/PNNL-17167.pdf 


\begin{tabular}{|c|c|c|c|c|}
\hline Project & Description & Goal/Scope & Key Participants & Timing \\
\hline & & $\begin{array}{l}\text { - Apply smart grid capabilities to support the } \\
\text { integration of a rapidly expanding portfolio of } \\
\text { renewable resources in the region"s }\end{array}$ & & \\
\hline $\begin{array}{l}\text { AEP gridSmart } \\
\text { Real-Time } \\
\text { Pricing- } \\
\text { Double Auction } \\
\text { Demonstration } \\
\text { Project }\end{array}$ & $\begin{array}{l}\text { PNNL-sponsored research } \\
\text { project focused on } \\
\text { understanding how } \\
\text { residential households might } \\
\text { adapt their electricity } \\
\text { consumption in response to } \\
\text { fluctuating 5-minute price } \\
\text { signals }\end{array}$ & $\begin{array}{l}\text { - Understand the potential benefits of real-time } \\
\text { pricing and double auction approach for impact on } \\
\text { system capacity and feeder capacity issues } \\
\text { - Understand the potential benefits of improving } \\
\text { wholesale purchases in the real-time (5-minute) } \\
\text { market and participation in a spinning reserve } \\
\text { market } \\
\text { - Understand the sensitivity of consumers to real-time } \\
\text { pricing. }\end{array}$ & $\begin{array}{l}\text { - PNNL } \\
\text { - AEP Ohio }\end{array}$ & $\begin{array}{l}2011- \\
2013\end{array}$ \\
\hline $\begin{array}{l}\text { Clean Energy } \\
\text { and Transactive } \\
\text { Campus }\end{array}$ & $\begin{array}{l}\text { Project funded by DOE and } \\
\text { the State of Washington to } \\
\text { "advance understanding and } \\
\text { broader deployment of } \\
\text { transactive energy } \\
\text { approaches in building } \\
\text { loads, renewable energy, } \\
\text { and other distributed energy } \\
\text { resources." } 6\end{array}$ & $\begin{array}{l}\text { - "[E]stablish a blueprint to replicate and scale up } \\
\text { transactive control methodologies for application in } \\
\text { buildings, campuses and communities across the } \\
\text { nation." }\end{array}$ & $\begin{array}{l}\text { - PNNL } \\
\text { - University of } \\
\text { Washington } \\
\text { - Washington State } \\
\text { University } \\
\text { - Case Western } \\
\text { Reserve University } \\
\text { - University of } \\
\text { Toledo } \\
\text { - NASA Glenn } \\
\text { Research Center }\end{array}$ & $\begin{array}{l}2016- \\
\text { present }\end{array}$ \\
\hline $\begin{array}{l}\text { Transactive } \\
\text { Energy } \\
\text { Network Demo }\end{array}$ & $\begin{array}{l}\text { Project funded by } \\
\text { Sustainable Development } \\
\text { Technology Canada to } \\
\text { link together three } \\
\text { microgrids (Toronto, Nova }\end{array}$ & $\begin{array}{l}\text { - "[D]emonstrate a smart and integrated transactive } \\
\text { energy (TE) network that will allow DERs to be } \\
\text { integrated technically and financially into electric } \\
\text { power systems." }\end{array}$ & $\begin{array}{l}\text { - Opus One Solutions } \\
\text { Energy Corporation } \\
\text { - Advanced } \\
\text { Microgrid Solutions } \\
\text { - Emera Maine } \\
\end{array}$ & $\begin{array}{l}\text { 2016- } \\
\text { present }\end{array}$ \\
\hline
\end{tabular}

${ }^{5}$ Pacific Northwest Smart Grid Demonstration Project. Technology Performance Report. Volume 1: Technology Performance. Battelle Memorial Institute. June 2015.

https://www.smartgrid.gov/files/TPR01Introduction.pdf

${ }^{6} \mathrm{https} / / /$ ggintegration.pnnl.gov/connectedcampus.asp

${ }^{7}$ Ibid

${ }^{8}$ https://www.sdtc.ca/en/projects/transactive-energy-network-for-clean-generation-energy-storage-electric-vehicle-charging-and-microgrid-integration/ 


\begin{tabular}{|c|c|c|c|c|}
\hline Project & Description & Goal/Scope & Key Participants & Timing \\
\hline & $\begin{array}{l}\text { Scotia, and upstate Maine) } \\
\text { into a transactive energy } \\
\text { framework }\end{array}$ & & $\begin{array}{l}\text { - Nova Scotia Power } \\
\text { Inc. } \\
\text { - Ryerson CUE- } \\
\text { Toronto Hydro } \\
\text { - Smarter Grid } \\
\text { Solutions } \\
\text { - Toronto Hydro } \\
\end{array}$ & \\
\hline $\begin{array}{l}\text { Retail } \\
\text { Automated } \\
\text { Transactive } \\
\text { Energy System } \\
\text { (RATES) }\end{array}$ & $\begin{array}{l}\text { California Energy } \\
\text { Commission-funded } \\
\text { program to develop and } \\
\text { pilot a retail transactive } \\
\text { energy platform with } 200 \\
\text { residential and small } \\
\text { commercial customers of } \\
\text { Southern California Edison. } \\
\text { Pilot is to incorporate } \\
\text { automated end device } \\
\text { controls and a two-way } \\
\text { subscription tariff }\end{array}$ & $\begin{array}{l}\text { - "Develop and pilot-test behind-the-meter load } \\
\text { management systems, operational strategies, and } \\
\text { retail tariff options that minimize the cost and } \\
\text { complexity of customer participation in electricity } \\
\text { markets } \\
\text { - Engage participants from any customer sector, but } \\
\text { focus on residential and small commercial } \\
\text { customers"9 }\end{array}$ & $\begin{array}{l}\text { - Universal Devices } \\
\text { Inc. } \\
\text { - TeMix Inc } \\
\text { - Southern California } \\
\text { Edison (SCE) } \\
\text { - California } \\
\text { Independent System } \\
\text { Operator (CASIO) } \\
\text { - OpenADR Alliance }\end{array}$ & $\begin{array}{l}\text { June } 2016 \\
\text { - March } \\
2019\end{array}$ \\
\hline TransactiveGrid & $\begin{array}{l}\text { LO3's blockchain-based } \\
\text { TransactiveGrid platform is } \\
\text { intended to enable peer-to- } \\
\text { peer energy transactions and } \\
\text { to facilitate price-based } \\
\text { control of distributed energy } \\
\text { resources. }\end{array}$ & $\begin{array}{l}\text { - Development of a "community energy market" } \\
\text { which would allow for peer-to-peer energy } \\
\text { transactions }\end{array}$ & - LO3 Energy & On-going \\
\hline
\end{tabular}

\footnotetext{
${ }^{9}$ http://www.temix.net/images/GFO-15-311_Retail Automated Transactive Energy System.pdf

${ }^{10}$ https://lo3energy.com/
} 


\section{Appendix B TE Challenge Scenario Technical Specification}

This Appendix presents the technical details of the TE Challenge Phase II Scenario. The full report documenting the multi-step collaborative development of the Scenario is in [1].

The Challenge Scenario is a collection of components including: a specific electric feeder with specified loads and generators, an event sequence (weather for one day), a time-of-use price schedule for the baseline (non-TE) case, a series of simulations that move from a nonTE baseline up to simulations with TE models chosen by each team, and an agreed upon set of common metrics for reporting results. These components are discussed more below.

\section{IEEE 8500 Grid}

The Institute of Electrical and Electronics Engineers (IEEE) 8500 reference distribution grid [2], shown in Figure Apdx B-1, was selected as the basis for the Challenge scenario. This 8500-node test feeder is a moderately large radial distribution feeder consisting of both low and medium voltage (MV) levels. The nodes occur at approximately 4,800 1-, 2-, and 3phase bus locations. The power flow is moderately difficult to solve at the specified load level. The test grid also exhibits approximately $10 \%$ power losses at peak load. The circuit contains $170 \mathrm{~km}$ of primary (MV) conductor. The circuit contains one set of regulators controlling the feeder voltage at the substation and three sets of voltage regulators along the line. The circuit also contains four capacitor banks, three that include per-phase capacitor control. The controlled capacitor banks monitor each phase separately, and each capacitor bank operates the respective capacitor on the same phase. The capacitor switches ON when the reactive power flow in the line is $50 \%$ of the capacitor size and switches OFF when the flow is $75 \%$ of the capacitor size in the reverse direction. Each controlled capacitor also includes voltage override where the capacitor turns $\mathrm{ON}$ at $0.9875 \mathrm{pu}$ and turns $\mathrm{OFF}$ at 1.075 p.u.

The test feeder makes two load model cases (secondary loading case models with residential loads connected to a $120 \mathrm{~V} / 240 \mathrm{~V}$ split-phase transformer in balanced and unbalanced configurations) available to users. The teams agreed to use a balanced configuration. Specifications for loads, PV generation, and batteries in houses at each meter were developed. As specified, the grid is a purely residential feeder, with a nominal peak load of 10.8 MW and 2.7 MVAr. Of the 1,977 houses on the feeder, all of them were configured with air conditioning (26.15 MW load), and $90 \%(1,777)$ of them with PV systems with total peak solar production of $6,755 \mathrm{~kW}$. In addition, 857 homes have batteries with a total storage capacity of 4,285 $\mathrm{kW}$ (although these were not used in the baseline configuration), and 1,013 homes have electric water heaters totaling $4,574 \mathrm{~kW}$. The grid definition and specific details of each house (thermal parameters and equipment), are contained in the inv8500.glm GridLab-D model file, available on a GitHub site managed by PNNL as part of their TE Simulation Platform (TESP) [3]. TE Challenge scenario files are maintained within the /examples/IEEE8500 directory of TESP Release 1.1 [4]. This directory has a readme.md file that explains the various model files, input files, data processing scripts, and results files. This directory serves as a repository of the TE Challenge scenario simulation files. 


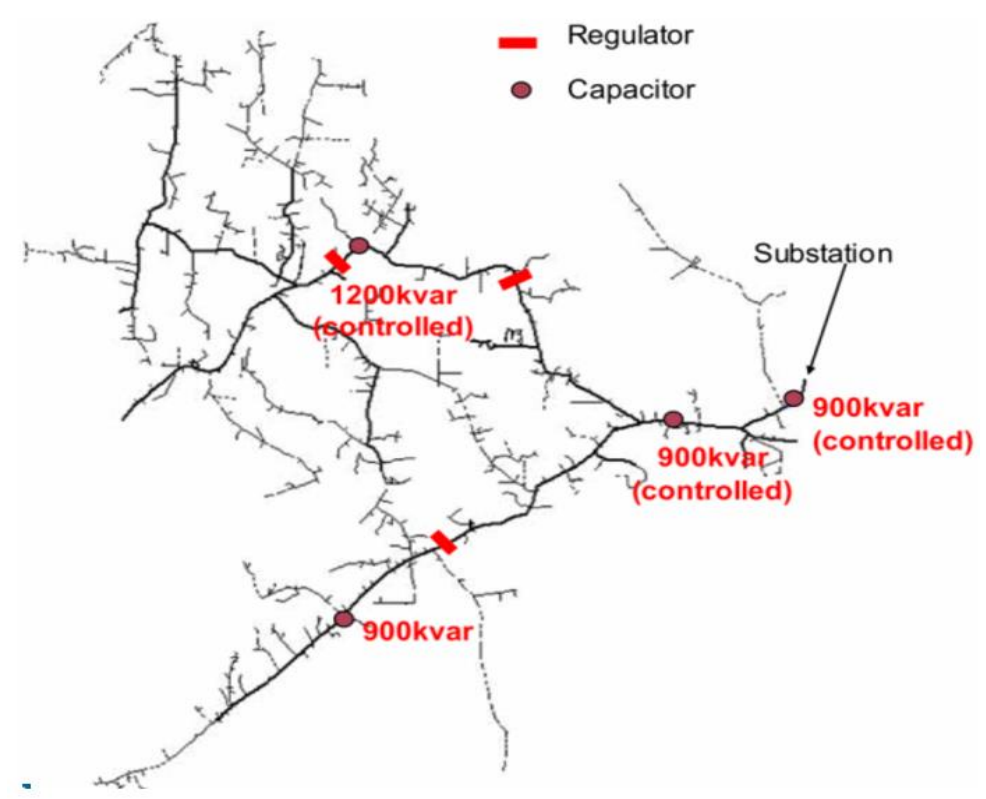

Figure Apdx B-1 IEEE 8500 grid schematic.

\section{Weather Scenario}

The High-penetration PV and Voltage Control scenario was originally written for a distribution system with a high penetration of PV systems and over-voltage conditions under sunny skies. The TE Challenge teams agreed to add a storm passing to induce voltage fluctuations and then to use transactive methods to incentivize controlled changes in load, generation or storage to mitigate the voltage imbalances. The single day scenario begins with clear sky conditions followed by PV generation dropping from full power to $10 \%$ power output in the 2:30 p.m. to 2:40 p.m. time window due to the storm front arrival. The overcast conditions last until a ramp back up to full sun from 4:00 p.m. to 4:30 p.m.

This scenario narrative is realized in simulation using the climate.csv weather input file [4] based on a sunny Tucson weather day with the addition of an artificial storm front per the narrative above. There is no time shift for the storm front passing over the feeder; every PV panel sees the same weather at the same time per above.

\section{Team Simulations}

Teams followed an implementation plan with four simulations in a progression to allow building up common understanding, validation by comparison of results, and refinement of the scenario components themselves. The progressive simulations were:

1. Baseline sunny day. The event day is run with no storm front. Electricity price is constant with no TE market interactions.

2. Adding the storm front. Simulation is repeated with the storm front weather file, with all else the same.

3. Adding dynamic price, without TE market. Teams may enable resources to be price responsive, but there are no TE exchanges.

4. Each team implements a TE model of their choice and applies it to the same feeder and weather event. 


\section{Price Data}

Teams agreed to use existing Tucson Electric retail tariffs and representative Tucson locational marginal pricing (LMP) data for a summer day. The Residential Basic Service flat rate tariff [5] was used for simulation Steps 1 and 2, with components given in Table Apdx B-1. The resulting "fixed rate" value used in the one-day TE Challenge simulation, 0.102 $\$ / \mathrm{kWh}$, is the sum of the 0 to $500 \mathrm{kWh}$ Energy Service Charge plus the Power Supply Charge.

Table Apdx B-1 Tucson Electric Residential Basic Service Tariff

\begin{tabular}{|l|l|l|}
\hline Tariff Component & Charge & Range \\
\hline Basic Service Fee & $\$ 13.00$ & \\
\hline Energy Service & $\$ 0.066152 / \mathrm{kWh}$ & $(0$ to 500$) \mathrm{kWh}$ \\
\hline & $\$ 0.081152 / \mathrm{kWh}$ & $(501$ to 1000$) \mathrm{kWh}$ \\
\hline & $\$ 0.086652 / \mathrm{kWh}$ & Over $1000 \mathrm{kWh}$ \\
\hline Power Supply & $\$ 0.035861 / \mathrm{kWh}$ & Summer value \\
\hline
\end{tabular}

The Residential Time-of-Use (TOU) rate [6] was used for Step 3. The TOU rate components are given in Table Apdx B-2Table Apdx B-1 Tucson Electric Residential Basic Service Tariff. For TE Challenge simulations, the on-peak or off-peak Power Supply Charge is added to the Energy Service Charge component.

Table Apdx B-2 Tucson Electric Residential Service Time-of-Use Tariff

\begin{tabular}{|l|l|l|}
\hline Tariff Component & Charge & Range \\
\hline Basic Service Fee & $\$ 10.00$ & \\
\hline Energy Service & $\$ 0.072152 / \mathrm{kWh}$ & $(0$ to 500$) \mathrm{kWh}$ \\
\hline & $\$ 0.081152 / \mathrm{kWh}$ & $(501$ to 1000$) \mathrm{kWh}$ \\
\hline & $\$ 0.086652 / \mathrm{kWh}$ & Over $1000 \mathrm{kWh}$ \\
\hline Power Supply & $\$ 0.066567 / \mathrm{kWh}$ & On-peak* \\
\hline & $\$ 0.026332 / \mathrm{kWh}$ & Off-peak \\
\hline
\end{tabular}

* Summer on-peak period is 3:00 p.m. to 7:00 p.m., Monday through Friday.

For PNNL TESP Gridlab-D users, the flat price and TOU tariffs are embedded in the GLM file. One can switch between basic and TOU by commenting/uncommenting near the top of the file, and all the meters reference those tariff schedules. LMP five-minute clearing price data [7] are based on the California Independent Service Operator real-time market prices for Tucson $^{11}$ for July 6, 2017, which has base prices around $20 \$ / \mathrm{MWh}$ and peaks around 50 $\$ / M W h$ in the afternoon. Day-ahead market hourly LMPs are also available for July 6-7 $7^{12}$. Use of the LMP real-time or day-ahead data is not required.

\section{Common Metrics}

To promote the ability to compare the results from simulations using different TE market and control approaches, the participating teams agreed to a set of common performance metrics. The metrics were derived from features of the PNNL TESP, American National Standards

\footnotetext{
${ }^{11}$ Taken from oasis.caiso.com Prices/Interval Locational Marginal Price at load node SG_LNODE13A.

${ }^{12}$ Taken from oasis.caiso.com Prices/Locational Marginal Prices with DAM option and SG_LNODE13A selected.
} 
Institute (ANSI) C84.1 [8], and IEEE 1366 [9]. Teams were permitted to ignore metrics not applicable to their simulations and to use additional metrics relevant to their results.

General guidelines:

- Save results in a text format, JavaScript Object Notation (JSON) (preferred) or common-separated values (CSV)

- Adjustable metrics interval, defaulting one minute

- If the simulation time step is shorter than the metrics interval, include minimum, maximum and average values within each interval. Integrated metrics (e.g., energy) are an exception

- Optionally, save all power flow data to CSV files by manually inserting recorder statements in the GridLab-D input file

- Separate PV output, battery, and load metrics at the same meter

- No time aggregation, as the use case covers a single day of operation

- Each metric should be associated with specific model components through metadata

- All measurements are time stamped

Specific requirements for base metrics to include in the output:

1. Economic

a. Wholesale price (defaults to the input LMP player file)

b. Cleared price(s) on the feeder

c. Price, quantity, and status (accepted, not accepted) for each bid

d. Revenue at each meter, separable by load and resource (see 4.f-k,m below)

2. Substation

a. Real and reactive power

b. Real and reactive energy

c. Real and reactive losses

3. At each feeder capacitor bank and voltage regulator

a. Count of control actuations

4. At each meter (i.e., house)

a. Voltage magnitude, line-to-neutral, averaged over all phases

b. Voltage magnitude, line-to-line, averaged over all phases ${ }^{13}$

c. For three-phase loads only, line-to-line voltage imbalance as defined in ANSI Standard C84.1: $100 \max _{p=1 . .3}\left(\left|V_{p}-V_{a v g}\right|\right) / V_{\text {avg }}[\%]$, where $V_{p}=$ phase voltage, and $V_{\text {avg }}$ is the average of the three phases.

d. Severity index (SI) for the fluctuation in $V_{a v g}$ on per-unit basis at uniform time step. Similar to an L2 norm; SI $=\sum_{k=1}^{n} \sqrt{\left(V_{k}-V_{k-1}\right)^{2}+1}$. This metric has also been used to quantify fluctuations in solar irradiance. IEEE 1453

Recommended Practice for the Analysis of Fluctuating Installations on Power Systems is less applicable because cloud-induced fluctuations are generally too slow.

\footnotetext{
${ }^{13}$ Because of the use of the balanced-secondary version of the IEEE 8500-node feeder, there is no secondary voltage unbalance at single-phase loads. The normalized line-to-neutral and line-to-line voltage magnitudes are equal. For such single-phase loads, $V_{\text {avg }}$ should be defined on a $120 \mathrm{~V}$ basis.
} 
e. Violations of ANSI C84.1 voltage limits at the meter. The duration of time in each range should be accumulated. An event count occurs when the voltage transitions from normal to A Range, or from A Range to B Range.

i. Total duration and event counts below $110 \mathrm{~V}$ (B Range)

ii. Total duration and event counts below $114 \mathrm{~V}$ (A Range)

iii. Total duration and event counts above $126 \mathrm{~V}$ (A Range)

iv. Total duration and event counts above $127 \mathrm{~V}$ (B Range)

v. Total duration and event counts below $10 \mathrm{~V}$ (Outage; none expected)

f. Total house load (real power)

g. Total heating, ventilation, and air conditioning (HVAC) load (real power)

h. Total water heater load (real power)

i. Solar inverter real and reactive power

j. Battery inverter real and reactive power

k. House air temperature, and its deviation from scheduled set point evaluated by:

i. Root mean squared deviation

ii. Maximum deviation

iii. Duration, average magnitude, and direction of the longest excursion

1. Water heater temperature and its deviation from scheduled set point

m. Total bill synchronized to the cleared market price ${ }^{14}$

5. Local utilization of green power, defined as the percentage of locally-generated green energy in the total energy consumed by all the customers connected to a feeder or in a microgrid. The sources of green energy considered are roof-top solar PV, but not batteries.

\section{References}

1. Holmberg, D., Burns, M., Bushby, S., Gopstein, A., "Transactive Energy Challenge Phase II Scenario," NIST Tech. Note 2021, 2018.

2. Arnatt D (2010) The IEEE 8500-node test feeder, Transmission and Distribution Conference and Exposition, IEEE PES. DOI: 10.1109/TDC.2010.5484381, and grid component files at: https://ewh.ieee.org/soc/pes/dsacom/testfeeders/8500node.zip

3. PNNL TE Simulation Platform http://tesp.readthedocs.io/.

4. PNNL TE Challenge scenario repository in TESP Release 1.1 (2018). Available: https://github.com/pnnl/tesp/releases/tag/v0.1.1.

5. Tucson Electric Residential Basic Service (2018) https://www.tep.com/wpcontent/uploads/2017/02/101-TRRES.pdf.

6. Tucson Electric Residential Time-of-Use (2018) https://www.tep.com/wpcontent/uploads/2017/02/102-TRREST.pdf.

7. CAISO real-time market prices for July 6, 2017, Tucson LMP five-minute clearing price data (2017) https://github.com/pnnl/tesp/blob/master/examples/players/lmp_value.csv.

8. ANSI C84.1-1982. American National Standard for Electric Power Systems and Equipment-Voltage Ratings $(60 \mathrm{~Hz})$.

9. IEEE 1366-2012 - IEEE Guide for Electric Power Distribution Reliability Indices.

\footnotetext{
${ }^{14}$ The 8500-node model as implemented here assumes net metering, with distributed energy resource (DER) disaggregation based on real power. An alternative would be to meter each DER separately from the house, so each could have its own tariff and bill.
} 